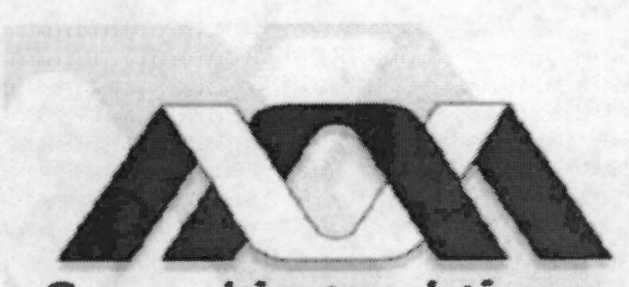

Casa abierta al tiempo UNIVERSIDAD AUTONOMA METROPOIITANA

\title{
Estudio del comportamiento del reactor de oxidación parcial de o-xileno a anhídrido ftálico
}

TESIS PARA OBTENER EL GRADO DE DOCTOR EN CIENCIAS EN INGENIERÍA QUÍMICA

PRESENTA

M. en C. Carlos Omar Castillo Araiza

\author{
ASESOR \\ Dr. Héctor Felipe López Isunza \\ SINODALES \\ Dr. Roberto Quintana Solórzano \\ Dr. José Antonio Muñoz Arroyo \\ Dr. Richard Steve Ruiz Martínez \\ Dr. Tomás Viveros García
}

Abril, 2008

UNIVERSIDAD AUTÓNOMA METROPOLITANA

UNIDAD IZTAPALAPA

DIVISIÓN DE CIENCIAS BÁSICAS

E INGENIERÍA 



\section{Agradecimientos......}

A Dios por darme la oportunidad de existir y ponerme siempre en el lugar y con las personas adecuadas.

A mis padres Alicia y Carlos, y hermanos Luis, Victor y Lesly. Porque gracias a su apoyo y consejo he llegado a realizar una de la metas mas reconfortantes en mi vida. Sabiendo que jamás existirá una forma de agradecer en esta vida de lucha y superación constante, deseo expresarles que mis ideales, esfuerzos y logros han sido también suyos y constituye el legado más grande une pudiera recibir. Con cariño, admiración y respeto.

A mi esposa, Inés. No es facil llegar, se necesita ahinco, lucha y deseo, pero sobre todo apoyo como el que he recibido durante este tiempo. Ahora mas que nunca se acredita mi carino, admiración y respeto. Gracias por lo que hemos logrado.

A Aurelia, Mario, Jazmin y Mario Jr. por su apoyo, comprensión y sobre todo motivación que nos han brindado a su hija, Inés, y a mi, lo cual ha sido una fuente de motivación para que hoy estemos viviendo este momento.

Un agradecimiento especial a Felipe López Isunza, asesor de este trabajo y amigo, que durante mas de 5 años ha compartido su conocimiento sobre la Ingenieria de Reactores y algunas experiencias de la vida cotidiana. Su comprensión y apoyo brindado hacen que el camino para terminar esta tesis no haya sido tan complicado. Seguir sus consejos, me permitió conocer otros horizontes que me han hecho crecer tanto personal como profesionalmente.

A mi mejor amigo, y ahora compadre, Victor (el flaco), ya que por más de 13 años hemos compartido tanto éxitos profesionales como problemas de la vida cotidiana. Gracias por 
permitirme conocer a Gaby, tu esposa, e hijos (Favio y Paolo) ya que ahora forman parte de esta amistad.

A todo el grupo de trabajo con el que me toco convivir: Urieh Tristán, Radai, Ángeh, Margarita, Celia, Noemi, Victor Hugo, Felipe, Hugo y Matias por su amistad y apoyo mostrado en todo momento.

A Lalo, Amapola, Ricardo y amigos del TESE, la Pandilla, las Moles, Rosa Iris, Ariana, Francis, lris, David y su adorable familia, por su apoyo y amistad brindada en este camino de éxitos y fracasos.

A los sinodales de este trabajo, Roberto Quintana, Antonio Muñoz, Tomas Viveros y Richard Ruiz, quienes con sus acertados comentarios y sugerencias enriquecieron el contenido de este trabajo, además de su apoyo brindado para que esta faceta en mi formación este finalizada.

A Hugo Jiménez Islas (Tecnológico de Celaya, Celaya México), Said Elnashaei (Universidad de Penn State, Pensilvania EUA), ya que las estancias en cada una de estas universidades me permitieron crecer profesionalmente.

A la UAMI por la oportunidad de permitirme alimentar mi formación profesional de su inagotable fuente de conocimientos.

A CONACyT por el apoyo económico otorgado para la realización de este trabajo, gracias.

A todas las persanas que me apoyaron y están disfrutando estos momentos conmigo, gracias. 


\section{Resumen}

En este trabajo se estudió el comportamiento en el estado estacionario y transitorio del reactor a escala industrial de oxidación parcial de o-xileno a anhídrido ftálico, con el proposito de predecir los perfiles de temperatura y concentración experimentales y conocer más sobre el comportamiento, tan complejo, de este sistema. Para esto, se llevaron a cabo estudios independientes sobre la hidrodinámica y el transporte de calor en ausencia de reacción química.

En el estudio hidrodinámico se evalúan varios modelos ya reportados en la literatura para predecir los perfiles de velocidad en el reactor en ausencia de reacción. Se encontró que el modelo de Darcy-ForchheimerNavier-Stokes, utilizando una ecuación empirica para describir los perfiles de fracción vacía y estimando la magnitud de los efectos viscosos e inerciales de datos experimentales de caida de presión, aparenta ser la aproximación más adecuada para predecir los perfiles de velocidad en el lecho de estudio.

En el estudio de transporte de calor sin reacción se estimo el coeficiente de transporte de calor en la pared (hw), la conductividad termica efectiva axial $\left(k_{\mathrm{ma}}\right)$ y radial $\left(k_{\mathrm{er}}\right)$. Para lograr esto, dos lechos, uno de laboratorio y otro industrial, fueros empacados con el mismo catalizador $y$ operados de forma casi-adiabática y no-adiabática. La operación del lecho a nivel laboratorio, en forma casi-adiabática, proporcionó datos experimentales para estimar $k_{\mathrm{az}}$ a varias velocidades de alimentación. Esta información se utilizó exitosamente para modelar ambos lechos, de laboratorio e industrial, operados de forma noadiabatica, $y$ asi estimar $h_{w} y k_{e r}$. Los ajustes de temperatura que se obtienen con la aproximación clásica, que modela el transporte de calor al considerar flujo uniforme en el lecho, se compararon cuando se consideran los campos de velocidad y el perfil radial de fracción vacía en lecho, así como su aproximación de capa límite. Los perfiles de temperatura observados en el lecho empacado se ajustan bajo cualquier 
caso, sin embargo, la diferencia entre los valores estimados de $k_{e r} y h_{w}$ fue mayor a un $10 \%$.

De las simulaciones del reactor a escala industrial, en el estado estacionario, se encontró que los perfiles de temperatura y concentración no se predicen adecuadamente cuando se consideran los campos de velocidad; sin embargo, cuando se consideró flujo pistón en el reactor $y$ se utilizan $k_{e z}, k_{\text {er }} y h_{w}$ estimados en ausencia de reacción y al considerar la hidrodinámica la predicción es más adecuada. Asimismo, para predecir el comportamiento observado en el reactor en el estado estacionario es necesario considerar la desactivación reversible e irreversible del catalizador, y utilizar un modelo pseudo-heterogéneo en dos dimensiones espaciales.

Finalmente, para la simulación del reactor catalítico en el estado transitorio se utilizó un modelo cinético basado en el esquema y parámetros cinéticos propuestos por Calderbank et al. (1977). Este modelo considera la desactivación reversible del catalizador $y$ se valida adecuadamente al ajustar los resultados observados en el reactor en el estado estacionario. Asi también, este modelo cinético predice de forma adecuada desde un punto de vista cualitativo el comportamiento transitorio del reactor. 


\section{Índice general}

Resumen

$$
\text { iv }
$$

fndice general

Indice de flguras

vi

Indice de tablas

xi

Notación

xviii

xix

Capitulo 1.

1. Introducción

Objetivos: general y particulares

Objetivo general

Objetivos particulares

Capítulo 2.

2. Hidrodinámica

2. 1 Hidrodinámica en un lecho empacado

2.2 Modelo hidrodinámico

2.2.1 Interacciones entre el sólido y fluido 19

2.2.2 Perfiles de fracción vacía

2.3 Hidrodinámica del sistema de estudio: resultados y discusion

2.3.1 Calda de presión

2.3.2 Perfiles de fracción vacía

2.3.3 Perfiles de velocidad

2.3.4 Evaluación de los distintos modelos hidrodinámicos que se emplean en la

literatura

2.3.5 Estudio de los distintos parámetros que intervienen en el modelo hidrodinámico 
3. Estudio del transporte de calor sin reacción

3.1 Transporte de calor en ausencia de reacción

3.2 Sistema experimental

3.3 Modelos de transporte de calor en el lecho empacado

3.3.1 El lecho operado de forma adiabática:

la aproximación clásica

3.3.2 El lecho operado de forma noadiabática: la aproximación clásica

3.3.2.1 Modelo en 1D espactal

3.3.2.2 Modelo en $2 D$ espaciales

3.4 Resultados y discusión sobre el estudio de transporte de calor

3.4.1 Transferencia de calor axial: Ia operación adiabática del lecho

3.4.2 Experimentos de transferencia de calor axial y radial: operación no-adiabática del Lecho

3.4.3 Estimación de los parámetros de transporte de calor: la aproximación clásica

3.4.3.1 Estimación del coeficiente global de transporte de calor (U)

3.4.3.2 Estimación del coeficiente de transporte de calor en la pared $h_{w}, y$ la conductividad térmica efectiva $k_{a r}$

3.4.4 Estimación de los parámetros de transporte de calor: incorporación de los perfiles de fracción vacia $y$ velocidad 85

3.5 Conclusiones y perspectivas

4. Estudio del comportamiento en estado estacionario del reactor de oxidación parcial de o-xileno a anhidrido 
4.1 Antecedentes sobre el estudio del reactor en el estado estacionario

4.2 Catalizador

4.2.1 Papel del oxígeno durante la reacción

4.2.2 Fenómenos de desactivación en la superficie del catalizador

4.3 Estudios cinéticos en la actualidad 110

4.4 Sistema experimental 112

4.5 Experimentos y resultados en el reactor 115

4.6 Modelos del reactor empleados en el presente estudio

4.6.1 Modelo pseudo-homogéneo en una dimensión espacial

4.6.2 Modelo pseudo-heterogéneo en una dimensión espacial

4.6.3 Modelo pseudo-homogéneo en dos dimenstones espaciales

4.6.4 Modelo pseudo-heterogéneo en dos dimensiones espaciales

4.6.5 Modelo cinético y los perfiles de desactivación

4.6.6 Modelo adimensional del reactor

4.7 Efecto de la desactivación del catalizador en los perfiles de temperatura y concentración

4.7.1 Efecto de 108 parámetros de $1 a$ Ecuación de desactivación del catalizador

4.7.2. Evaluación de los modelos del reactor: el efecto de la desactivación del catalizador

4.8 Simulación del reactor al emplear los parámetros de transporte de calor estimados sin reacción.

4.8.1 Sensibilidad de $h_{w} y k_{\text {er }}$ al ser 


\section{estimados de dos maneras: de la forma clásica y considerando la hidrodinámica \\ 4.8.2 Simulación del reactor usando los parámetros de transporte de calor estimados considerando isotropía en el lecho

5. Una introducción al comportamiento transitorio del reactor de oxidación parcial de o-xileno a anhidrido ftálico

\subsection{Antecedentes}

5.2 Modelo cinético de la oxidación de ox a AF 5.2.1 Validación del modelo cinético en estudios en estado estacionario

5.3 Evaluación del modelo cinético en el estudio transitorio del reactor

Capitulo 6.

6. Conclusiones

\section{Referencias Bibliogräficas}

A. Permeabilidad del lecho: $k(\varepsilon)$ y $k_{2}(\varepsilon)$

B. Correlaciones para evaluar la fracción vacía

C. Predicción de los perfiles de velocidad empleando CFD

D. Efecto de los perfiles de fracción vacía en la predicción de los perfiles de velocidad

E. Efecto de la variación de la densidad y viscosidad del fluido en la predicción del perfiles de velocidad

F. Dinámica de los perfiles de velocidad 
G. Impacto del perfil de velocidad en la predicción del perfil de temperatura

H. Evaluación de algunos modelos cinéticos

I. Esquema cinético propuesto

J. Efecto de los parámetros de transporte de calor y masa en los perfiles de temperatura y concentración 228

K. Predicción de la propuesta SIM-1 a diferentes 235 condiciones de operación 
Índice de figuras

Figura 2.1. Fotografía que describe el arreglo de las esferas de catalizador en el interior de un tubo de acrilico del mismo diámetro que el reactor a escala industrial. a) Negativo de la vista lateral, b) vista superior y c) vista lateral.

Figura 2.2. a) Comparación entre: la caída de presión experimental, la ajustada en este trabajo y la predicha con diferentes correlaciones propuestas en la literatura. b) Diagrama de paridad.

Figura 2.3. Comparación entre los datos de calda de presión con la correlación ajustada $y$ otras correlaciones: a) $R e_{p}$ de 25 a $785 ;$ y b) $R e_{p}$ de 25 a 160 .

Flgura 2.4. Predicción de los perfiles de fracción vacía usando diferentes correlaciones.

Figura 2.5. a) Predicciones de la permeabilidad y b) el número de Darcy.

Figura 2.6. Predicción de los perfiles radiales de velocidad axial, para diferentes $R e_{p}$ : a) modelo $1-A$ y b) modelo 1-B.

Figura 2.7. Predicción de los campos de velocidad axial a 10 largo del radio, empleando diferentes $\operatorname{Re}_{\mathrm{p}} \mathrm{y}$ el modelo $1-C$.

Figura 2.8. Predicción de los campos de velocidad axial a 10 largo del radio para diferentes $R e_{p}$ : a) modelo 1 D y b) modelo $1-E$.

Figura 2.9. Predicción de los campos de velocidad axial a 10 largo del radio con los modelos $1-\mathrm{A}$ a $1-\mathrm{E}$ : a) $R e_{p}=25$ y b) $R e_{p}=785$.

Flgura 2.10. Predicción de los campos de velocidad axiál a 10 largo del radio estimando: a) la viscosidad efectiva $y$ b) los parámetros $\alpha$ y $\beta$.

Figura 2.11. Comportamiento de la viscosidad efectiva. 
Figura 2.12. Predicción de los campos de velocidad axial a lo largo del radio, para diferentes $\mathrm{Re}_{\mathrm{p}}$ : a) modelo 2-A Y b) modelo 2-B.

Figura 2.13. Comparación de las predicciones de los campos de velocidad axiales a lo largo del radio con el. modelo 2-C y el modelo $2-D$.

Blgura 2.14. Predicciones de los campos de velocidad a lo largo del radio para el modelo $2-F$ : a) la componente axial y b) la componente radial.

Flgura 2.15. Comparación entre los modelos 2-G y 2-F a dos diferentes flujos.

Figura 3.1. Relación estadistica entre los parámetros de transporte de calor en un lecho empacado.

B1gura 3.2. Sistemas experimentales usados en el estudio de transporte de calor en el lecho de laboratorio. a) En la dirección axial; b) en la dirección axial y radial.

Figura 3.3. Arreglo de los termopares y termopozos a 10 largo del lecho.

Figura 3.4. Comparación entre los perfiles de temperatura experimental y ajustado en la dirección axial al estimar la $k_{a z}$.

Figura 3.5. a) Dependencia del Pe con el número de Reynolds $R e_{p}$. b) IInea de paridad del PE. (A) Ajuste, (ㅁ) Correlación modificada de $Y K W,(0)$ Correlación de YKW (1960), ( $\Delta)$ Bey Y Eigenberger (2001) y (0) Demirel et al. (2000).

Figura 3.6. Perfiles de temperatura experimentales en la pared interna del lecho a diferentes números de $\mathrm{Re}_{\mathrm{p}}$.

Figura 3.7. Perfil de temperatura radial en función del $R_{p} a$ diferentes posiciones axiales del lecho. a) $z=12$ $\mathrm{cm}$ y b) $\mathrm{z}=25 \mathrm{~cm}$. 
Figura 3.8. Perfiles de temperatura experimentales, en el lecho a escala piloto, en la dirección radial para un $R e_{p}=630$ (Corrida 10).

Figura 3.9. Comparacion entre los resultados experimentales y los ajustes del modelo pseudo-homogéneo $1 D$. a) perfiles de temperatura axial a diferentes $R e_{p} y$ b) el perfil de temperatura radial a $R e_{p}=25$.

Figura 3.10. Comparación entre los perfiles de temperatura observados y los ajustes del módelo pseudohomogéneo $1 D$ al estimar $U$, para el lecho a escala industrial.

Figura 3.11. Estimación del coeficiente global de transporte calor en función del $\mathrm{Re}_{\mathrm{p}}$.

Figura 3.12. Comparación de los perfiles de temperatura experimentales $y$ los obtenidos al emplear distintas correlaciones para calcular los números de Peclet y Biot. (A) Experimentos, (0) $\mathrm{Pe}=6, B_{1 w}=0.38[116]$, (a) $\mathrm{Pe}=18.62, \mathrm{~B}_{\mathrm{iw}}=1.05-$ Demirel et al. $(2000)[115](0) \quad P e=16.4, B_{1 w}=0.8-$ Bey et al. (2001) [33] ( $\Delta$ ) $\mathrm{Pe}=17.82, B_{i w}=0.83$ [14] y $(\nabla$ ) estimados en este trabajo (Caso $1-B$ ).

Figura 3.13. Ajuste de los perfiles de temperatura, estimando unicamente $h_{w}$ para un $R e_{p}=25$. En las direcciones: a) radial $y$ b) axial.

Figura 3.14. Ajuste de los perfiles de temperatura estimando $h_{w} y k_{e r}$ para un $R e_{p}=25$. En las direcciones: a) radial y b) axial.

Figura 3.15. Ajuste de los perfiles de temperatura estimando únicamente $h_{k}$ para un $R e_{p}=50$. En las direcciones: a) radial y b)axial.

Figura 3.16. Ajuste de los perfiles de temperatura estimando. $h_{w} y \quad k_{e r}$ para un $R e_{p}=56$. En las direcciones: a) radial y b)axial. 
Figura 3.17. Ajuste de los perfiles de temperatura estimando unicamente $h_{w}$ para un $R e_{p}=160$. En las direcciones: a) Radial y b)axial.

Figura 3.18. Ajuste de los perfiles de temperatura estimando hw $y$ kor para un $R_{p}=160$. En las direcciones: a) radial y b) axial.

F1gura 3.19. Ajuste de los perfiles de temperatura estimando únicamente $h_{*}$ para un $R e_{p}=630$. En las direcciones: a) radial y b) axial.

Figura 3.20. Ajuste de los perfiles de temperatura estimando $h_{w} y$ ker para un $R_{p}=160$. In las direcciones: a) radial y b) axial.

Figura 4.1. Sitios presentes de $\mathrm{V}_{2} \mathrm{O}_{5}$ en la superficie del catalizador.

71gura 4.2. Esquemas cinéticos de la oxidación de OX a AF publicados por varios investigadores (ver Tabla 4.1).

81gura 4.3. Diagrama de la planta piloto para la oxidación parcial de $O X$ a $A F$.

Figura 4.4. Perfiles de temperatura en el reactor alcanzados desde el arranque del reactor al primer estado estacionario. a) Axial y b) radial.

Figura 4.5. Perfiles de concentración de OX Y AF para las condiciones del Exp-1.

Figura 4.6. Perfiles de temperatura a las condiciones del Exp-3 a diferentes tiempos de reaccion. 120

Figura 4.7. Conexión entre la fase sólido y gas.

Figura 4.8. Esquema de reacción de Calderbank et al.

Figura 4.9. Efecto del parámetro $q$ de la función de desactivación. (a) En los perfiles de concentración y temperatura y (b) en el valor de la función de desactivación $\mathrm{a}(\mathrm{z})$, en función de la posición axial del reactor: 
Figura 4.10. Efecto del parámetro $p$ de la función de desactivación. (a) En los perfiles de concentración y temperatura $y(b)$ en el valor de la función de desactivación $a(z)$, en función de la posición axial del reactor.

Figura 4.11. Efecto de la desactivación del catalizador en la predicción del modelo HOM-1D a las condiciones del Exp-1. (a) Los perfiles de temperatura; (b) los perfiles de concentración.

Figura 4.12. Evaluación de los modelos HOM-2D y HOM-2D-HID en la predicción del Exp-1. a) Temperatura y. b) concentración.

Figura 4.13. Predicción del Exp-1 con el modelo HET-ID considerando dos diferentes perfiles de desactivación. a) Temperatura y b) concentración.

Figura 4.14. Predicción de los perfiles de temperatura y concentración del EXP-1 empleando el modelo HET2D-PH. a) Temperatura y b) concentración.

F1gura 4.15. Predicción del perfil de temperatura radial del Exp-1 con el modelo HET-2D-PH.

Figura 4.16. Comparación de los perfiles de actividad empleando diferentes modelos del reactor para predecir el EXP-1.

Figura 4.17. Predicción del modelo HET-2D-PH al emplear diferentes condiciones de operación.

Figura 4.18. Predicción de los modelos homogéneos a dos diferentes condiciones de operación. a) HOM-1D Y b) HOM-2D.

Figura 4.19. Predicción del modelo al usar los parámetros de transporte de calor estimados de la forma clásica y con la hidrodinámica. Considerando NoIsotropia en el reactor; el Exp-1; el modelo HET-2D. 
Figura 4.20. Predicción del modelo al usar los parámetros de transporte de calor estimados de la forma clásica y con hidrodinámica. Considerando: noisotropia en el reactor; el Exp-3; el modelo HET - $2 D$.

Figura 1.21. Predicción del modelo al usar los parámetros de transporte de calor estimados de la forma clásica $y$ con hidrodinámica, considerando isotropía en el reactor; el Exp-3; el modelo HET-2D.

Figura 5.1. Desplazamiento de los perfiles de temperatura a) al variar la $T_{b}$ de $663 \mathrm{~K}$ a $681 \mathrm{~K}$, b) al variar 1a $T_{b}$ de $681 \mathrm{~K}$ a $663 \mathrm{~K}$ y c) la comparación del estado estacionario inicial y final a la misma $T_{b}$ $(663 \mathrm{~K})$.

Figura 5.2. Comportamiento de la temperatura en el interior del reactor a cambios reversibles de la temperatura del baño.

Pigura 5.3 Ajuste de SIM-1 a las condiciones del Exp-1. a) Perfiles de temperatura y concentración $y$ b) perfil de sitios presentes en la superficie del catalizador.

Figura 5.4 Ajuste de SIM-2 a las condiciones del Exp-1. a) Perfiles de temperatura $y$ concentración $y$ b) perfil de sitios presentes en la superficie del catalizador.

Figura 5.5. Predicción de los perfiles de temperatura al incrementar la temperatura del baño $10 \mathrm{~K}$ (1 $\mathrm{K} \mathrm{X}$ min); y emplear el modelo cinético de Calderbank et al.

Figura 5.6. Predicción de los perfiles de temperatura al incrementar la temperatura del baño $10 \mathrm{~K}$ (1 $\mathrm{K} x$ $\min ): E_{\mathrm{ao}}=20,00 \mathrm{kcal} / \mathrm{kgmol}$. 
Figura 5.7. La predicción de los perfiles de temperatura al incrementar la temperatura del baño $10^{\circ}\left(1^{\circ} \mathrm{x}\right.$ min) y utilizar la propuesta SIM-1: $E_{20}=15,00$ $\mathrm{kcal} / \mathrm{kgmol}$. 


\section{Índice de tablas}

Tabla 2.1. Datos geométricos y físicos de los lechos: de 24 laboratorio y a escala industrial

Tabla 2.2. Condiciones de operación a las que se quieren los perfiles velocidad

Tabla 2.3. Diferentes derivaciones del modelo 1-E

Tabla 3.1. Condiciones experimentales

Tabla 3.2. Valores estimados del número de Peclet $y k_{a z} \quad 74$

Tabla 3.3 Valores estimados de los parámetros de 89 transporte de calor

Tabla 4.1. Diferentes etapas de reaccion consideradas en la literatura, de acuerdo a la Figura 4.2

Tabla 4.2 Caracteristicas del reactor y del catalizador 113

Tabla 4.3. Condiciones experimentales 115

Tabla 4.1. Parámetros cinéticos y calores de reacción 128

Tabla 4.5. Parámetros empleados en la simulación del reactor 131 


\section{Notación}

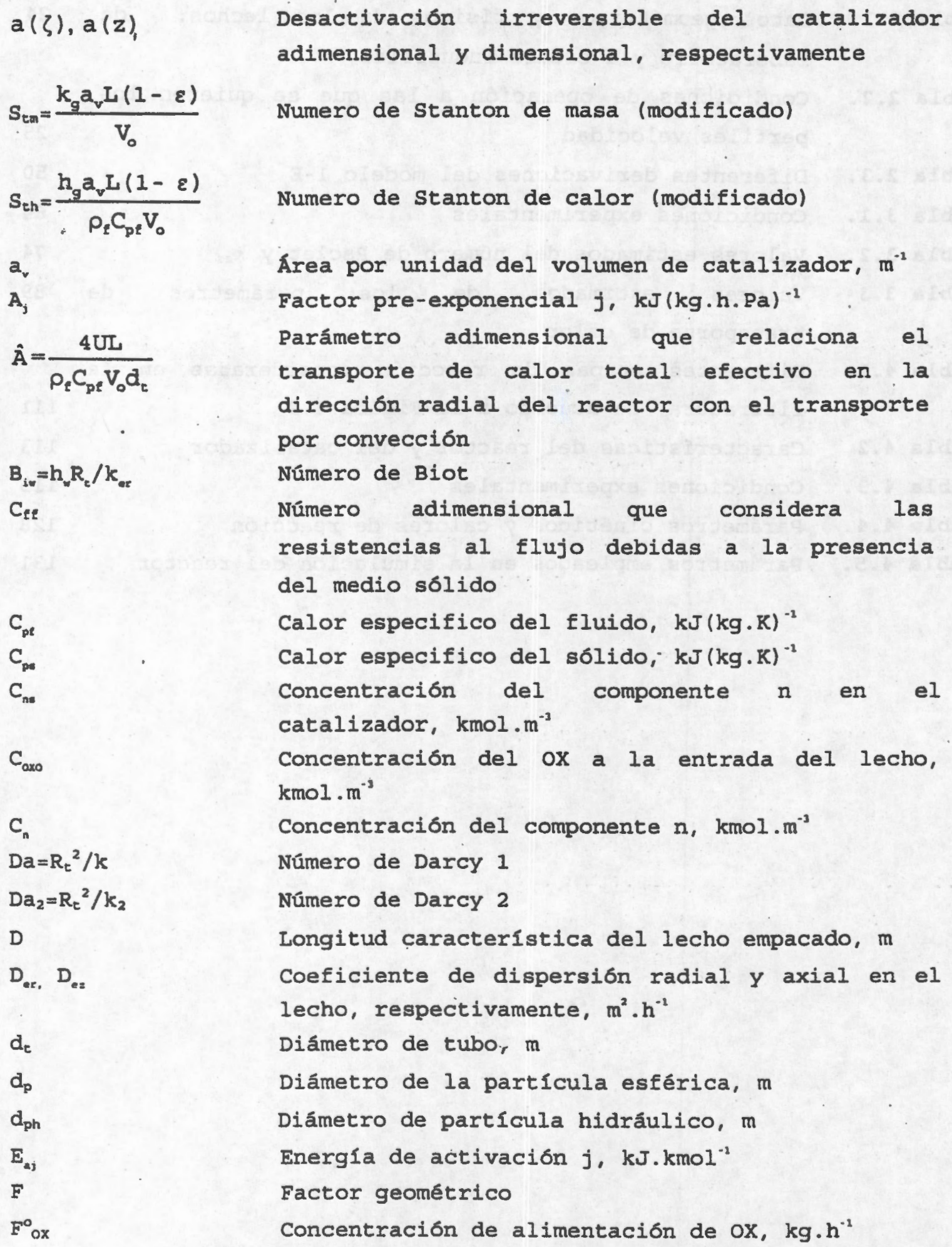


9

\section{$\mathbf{g}$}

$G=v_{0} p_{t}$

h.

h.

k

$k, k_{\mathbf{z}}$

$k_{\text {ex }}^{0}$

$\mathbf{k}_{\text {e }}$

$\mathbf{k}_{\mathrm{E}}$

k.

$\mathbf{k}_{\text {ez }}$

k.

k。

$\mathbf{k}_{\boldsymbol{t}}$

$\mathbf{k}_{\mathbf{j}}$

$k^{\prime},=k_{1} / k_{40}$

I.

P

$P_{n}$

$P_{r}=\mu_{l} C_{p e} / k_{q}$

$P e_{h z}=P e_{z}=G C_{p f} L / k_{e z z}$

$\mathrm{Pe}=\mathrm{GC}_{\mathrm{pt}} \mathrm{R}_{\mathrm{e}} / \mathrm{k}_{\mathrm{eqz}}$

$\mathrm{Pe}_{\mathrm{w}}=\mathrm{V}_{\mathrm{o}} \mathrm{L} / \bullet \mathrm{D}_{\text {ea }}$

$P e_{m e}=R_{r}^{2} V_{0} / L \bullet D_{e r}$

$P e_{n r}=R_{T}^{2} G C_{p l} / L k_{e r}$

$q_{0}$
Aceleracion normal de la gravedad, m.h ${ }^{-2} \quad(9.8$

m. $\operatorname{seg}^{-2}$ )

Vector de la gravedad, $\mathrm{m} \cdot \mathrm{h}^{-2}$

Flujo másico, $\mathrm{kg} \cdot \mathrm{m}^{-2} \cdot \mathrm{h}^{-1}$

Coeficiente de transferencia de calor en la pared, $k J \cdot m^{-2} \cdot h^{-2} \cdot K^{-2}$

Coeficiente de transferencia de calor entre la particula $y$ el fluido, $\mathrm{kJ} \cdot \mathrm{m}^{-3} \cdot \mathrm{h}^{-2} \cdot \mathrm{k}^{-1}$

Tensor de permeabilidad, $\mathrm{m}^{2}$

Permeabilidad en el lecho, $\mathrm{m}^{2}$

Conductividad térmica del lecho a flujos bajos $\left(R e_{p}<5\right), \mathrm{kJ} \cdot \mathrm{m}^{-1} \cdot \mathrm{h}^{-2} \cdot \mathrm{K}^{-1}$

Conductividad térmica efectiva, $\mathrm{kJ} \cdot \mathrm{m}^{-2} \cdot \mathrm{h}^{-1} \mathrm{~K}^{-1}$

Conductividad térmica del fluido, $\mathrm{kJ} \cdot \mathrm{m}^{-2} \cdot \mathrm{h}^{-1} \mathrm{~K}^{-1}$

Conductividad térmica efectiva axial, $\mathrm{kJ} \cdot \mathrm{m}^{-3} \cdot \mathrm{h}^{-1} \mathrm{~K}^{-3}$

Conductividad térmica efectiva radial, $\mathrm{kJ} \cdot \mathrm{m}^{-1} \cdot \mathrm{h}^{-2}$ $\mathbf{K}^{-1}$

Coeficiente de transferencia de masa entre la particula $y$ el fluido, $\mathrm{m} \cdot \mathrm{h}^{-1}$

Constante cinética del paso de oxidación, $\mathrm{m}^{3} \cdot \mathrm{h}^{\circ}$

${ }^{3} . \mathrm{kg}_{\text {eat }}{ }^{-3}$

Constante cinética del paso de reducción, $\mathrm{m}^{3} \cdot \mathrm{h}^{\text {* }}$

${ }^{1} . \mathrm{kg}_{\text {eat }}{ }^{-1}$

Constante cinética, para $j: 1, \ldots, 6, \mathrm{~m}^{3} \cdot \mathrm{h}^{-1} \cdot \mathrm{kg}_{\text {cat }}{ }^{-1}$

Parámetro cinético adimensional, para j: $1, \ldots, 6$

Longitud del lecho, $m$

Presión, $\mathrm{kg} \cdot \mathrm{m}^{-1} \cdot \mathrm{h}^{-2}$

Presión parcial del hidrocarburo $\mathrm{n}, \mathrm{Pa}$.

Número de Prandtl

Número de peclet axial de calor

Numero de Peclet de calor

Número de Peclet axial de masa

Número de Peclet radial de masa (modificado)

Número de Peclet radial de calor (modificado)

Flux de calor, $\mathrm{kJ} \cdot \mathrm{m}^{-2} \cdot \mathrm{h}^{-3}$ 


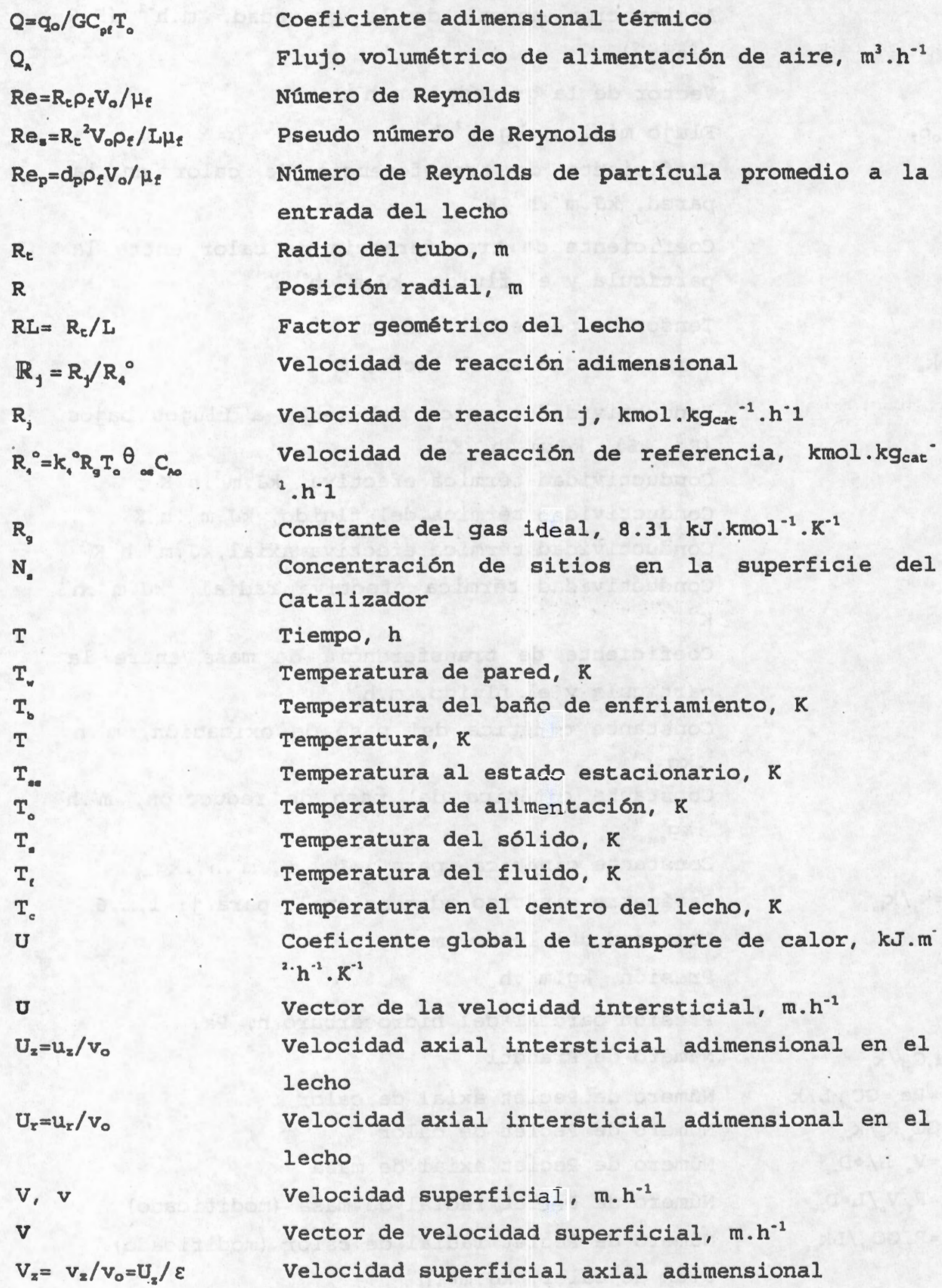




$$
\begin{aligned}
& v_{r}=v_{r} / v_{0}=U_{r} / \varepsilon \\
& v_{0}, v_{0}, V_{20}, v_{20} \\
& v_{p} \\
& W=\rho_{s} C_{m} / \rho_{t} C_{p t} \\
& Y_{w}=T_{w} / T_{0} \\
& Y=T / T \text { 。 } \\
& Y_{\infty}=T_{\text {en }} / T_{0} \\
& Y_{0}=T_{0} / T_{0} \\
& \mathrm{X}_{\mathrm{n}}=\mathrm{C}_{\mathrm{n}} / \mathrm{C}_{\infty} \\
& x_{n=0}=C_{n} / C_{n} \\
& x_{\text {no }} \\
& \mathbf{x}_{\text {ne: }} \\
& x, z
\end{aligned}
$$

Letras griegas

$$
\begin{aligned}
& \tau=\mu_{f} t / \rho_{t} R_{t}^{2} \\
& \tau=t \nu_{z} / R_{t}^{2} \\
& \tau_{1}=\tau / V_{0} \\
& \tau_{2}=\frac{\rho_{t} C_{p t}}{\rho_{g} C_{p t}} L / V_{0} \\
& \zeta=z / L \\
& \xi=r / R_{t} \\
& \xi_{u} \\
& \tilde{\mu} \\
& \mu_{f}
\end{aligned}
$$

Velocidad superficial radial adimensional

Velocidad superficial del fluido a la entrada del lecho, $\mathrm{m} \cdot \mathrm{h}^{-1}$

Volumen de la superficie de la particula, $\mathrm{m}^{3}$ Relación entre la capacitancia del solido y del Eluido

Temperatura adimensional en la pared

Temperatura adimensional

Temperatura adimensional al estado estacionario

Temperatura adimensional del catalizador

Concentración adimensional de la especie $n$ en la fase gas

Concentracion adimensional de la especie $\mathrm{n}$ en la superficie del catalizador

Concentración adimensional de la especie n a la entrada del lecho

Concentración adimensional de la especie $n$ en el estado estacionario

Posición axial en el lecho, m

Tiempo adimensional en los modelos hidrodinámicos

Tiempo adimensional en los modelos de transporte de calor sin reacción

Tiempo caracteristico en el balance del reactor en la fase gas y de masa en la fase sólida

Tiempo característico en el balance de calor en el catalizador

Posición axial adimensional

Posicion radial adimensional

Vector de la variable independiente

Viscosidad efectiva, $\mathrm{kg} \cdot \mathrm{m}^{-1} \cdot \mathrm{h}^{-1}$

Viscosidad del fluido, $\mathrm{kg} \cdot \mathrm{m}^{-1} \cdot \mathrm{h}^{-1}$ 


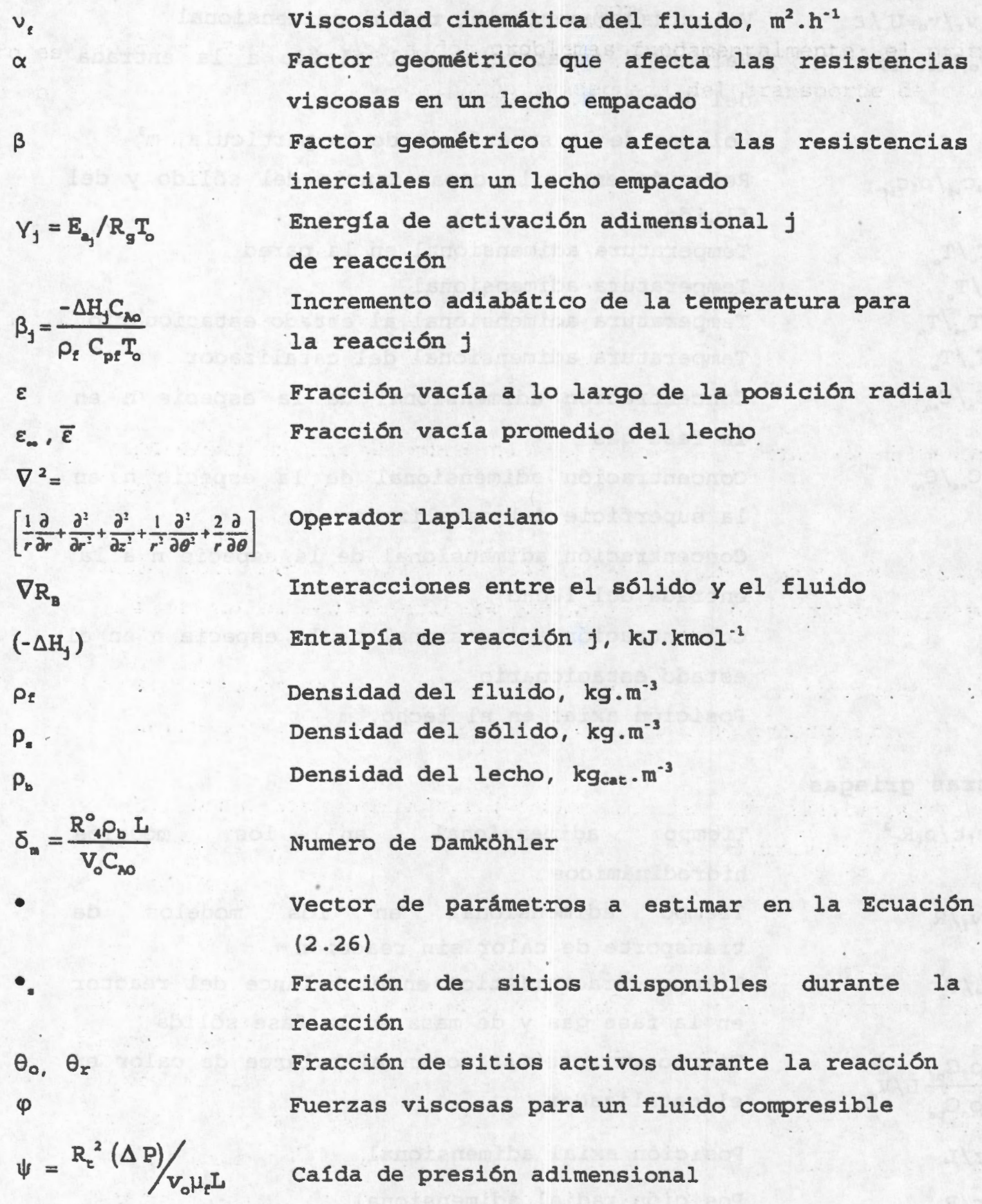

Abreviaturas

AF

AM
Anhidrido ftálico

Anhídrido maleico 
CFD

DRX

EDP

EDO

FT

FT-IR

HOM-1D

HOM-2D

HET - 1D

HET - 2D

HET-2D-PC

HET - 2D-PH

ox

OT

SIM-1

SIM-2

TPR

TPO

V-NMR

XPS

1D

2D
Dinámica de fluidos computacional

Difracción de rayos $x$

Ecuaciones diferenciales parciales

Ecuaciones diferenciales ordinarias

Ftálida

Espectroscopia de infrarrojo - transformada de Fourier

Modelo pseudo-homogéneo en una dimensión espacial

Modelo pseudo-homogéneo en dos dimensiones espaciales

Modelo pseudo-heterogéneo en una dimensión espacial

Modelo pseudo-heterogéneo en dos dimensiones espaciales

Modelo pseudo-heterogéneo en dos dimensiones, utilizando los parámetros de transporte de calor estimados de la forma clásica.

Modelo pseudo-heterogéneo en dos dimensiones, utilizando los parámetros de transporte de calor estimados al considerar la hidrodinámica.

o-xileno

O-tolualdehido

Simulación dinámica correspondiente al caso 1 simulación dinámica correspondiente al caso 2 Reducción programada con temperatura Oxidación programada con temperatura Resonancia magnética nuclear en estado sólido Espectroscopia foto-electrónica de rayos $\mathrm{X}$ Una dimensión espacial

Dos dimensiones espaciales 


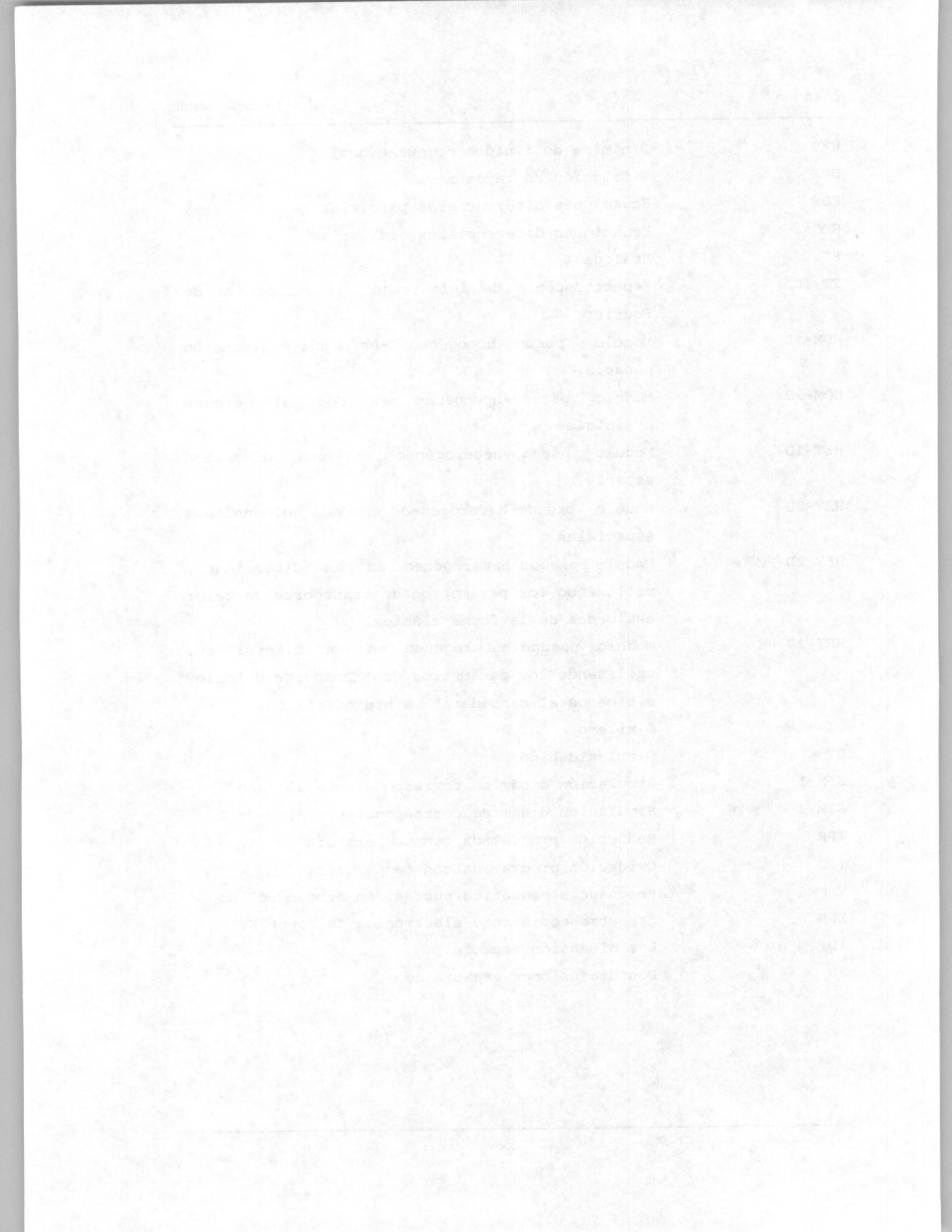




\section{Capítulo 1.}

\section{Introducción}




\section{Introducción}

Desde 1919 algunos de los estudios tebrico-experimentales en reactores de lecho fijo se han dirigido a aquellos que operan en forma noisotérmica y no-adiabática, algunos de los cuales operan reacciones altamente exotérmicas, como la oxidación parcial de hidrocarburos [127]. Estos reactores pertenecen a una clase que se distingue por presentar gradientes de temperatura y concentración apreciables, y una alta sensibilidad a cambios pequeños en las condiciones de operación $[10,12,14-16,18,19,21-23]$. En este trabajo se estudia la oxidación parcial de o-xileno (OX) a anhidrido ftalico (AF), utilizando un catalizador industrial, no poroso y esférico. Compuesto de $\mathrm{V}_{2} \mathrm{O}_{5}$ externamente depositado sobre $\mathrm{TiO}_{2}$ (fase anatasa), denotado como $\mathrm{V}_{2} \mathrm{O}_{5} / \mathrm{TiO}_{2}$. Debido a la alta exotermicidad de esta reacción la relación de diámetro de tubo a particula en este reactor es alrededor de 3.

Se sabe que el AF es uno de los productos más importantes en la síntesis orgánica moderna por su utilización en todo el mundo y su producción actual es mayor a 300,000 toneladas/año [20]. Generalmente esta reacción procede muy cercana a la presion atmosferica, y en un rango de temperaturas de 633 a $693 \mathrm{~K}$, dando una completa conversión de OX $y$ un rendimiento a $A F$ de 70 a $80 \%$. La mayor cantidad de productos intermediarios la conforman el o-tolualdehido (OT), y la ftalida (FT), y como productos secundarios se encuentran los óxidos de carbón (co y $\left.\mathrm{CO}_{2}\right), y$ el anhidrido maleico (AM).

Actualmente es claro que el proponer un modelo adecuado, que considere los distintos fenómenos catalíticos y de tránsporte de momento, calor y masa, es clave para llevar a cabo el diseño eficiente de un reactor $y$ para la optimización de unidades ya existentes. Con esta idea, trabajos teoricos y experimentales en el reactor de estudio $[10,12,13,14-19,21$ 36] han tenido por objetivo desarrollar modelos para describir el estado estacionario y el comportamiento transitorio; sin embargo, éstos no han sido exitosos debido a la gran complejidad que representa describir la fuerte interacción entre las reacciones altamente 
Copitulo 1. Introducción.

exotérmicas y el transportes de momento, masa y calor dentro del reactor. Esto se debe a dos problemas fundamentalmente; el primero está relacionado con la descripción inadecuada del transporte de calor en el lecho empacado, lo cual es característico en un sistema que presenta una baja relación de $d_{k} / d_{p}$, menor a $10[14,19,27]$; el segundo se debe a la incertidumbre en la descripción de la cinética de reacción [1419,23,25,31-34]. A la fecha, las fallas más comunes en la prediccion del comportamiento observado en el estado estacionario $y$ el transitorio de este reactor son:

a) La omisión de los perfiles de velocidad tanto en la simulación de los procesos de transporte de calor puros, como aquellos existentes durante la reacción.

b) Ia alta sensibilidad que presentan los parámetros de transporte de calor en la descripción del transporte de calor durante la reacción.

c) La predicción errónea de la magnitud y localización del punto caliente asi como la amplitud del perfil de temperatura.

d) El hecho de que prácticamente no hay un desplazamiento de los perfiles de temperatura durante los cambios dinámicos en las condiciones de operación.

En base a los comentarios anteriores, esta investigación se dirige a resolver estos problemas dando un particular punto de vista de como se deben abordar. Para ello se desarrolla un estudio sobre la hidrodinámica y el transporte de calor en ausencia de reacción. La validación de estos estudios se llevo a cabo al predecir el comportamiento observado del reactor a escala industrial durante la reacción en el estado estacionario y transitorio.

En el capitulo 2 se realiza un estudio sobre la hidrodinámica en el lecho empacado en ausencia de reacción. Se estudia el efecto de diferentes modelos hidrodinámicos reportados en la literatura, los 
perfiles de fracción vacía, la caida de presión y los fenómenos inerciales $y$ viscosos en la predicción de los campos de velocidad.

En el capitulo 3 se desarrolla un estudio teorico-experimental del transporte de calor en dos lechos empacados, uno a nivel laboratorio $y$ otro industrial, en ausencia de reacción. Este estudio permitió estimar los parámetros de transporte de calor, $h_{w}, k_{e x}, y k_{e z}$, que son utilizados en la simulación del reactor.

En el capítulo 4 se desarrolla un estudio térico en el estado estacionario del reactor industrial utilizado en la oxidación de ox a AF. Se estudia el efecto de la desactivación del catalizador, de los parámetros de transporte de calor y de la hidrodinámica, en la predicción de los perfiles de temperatura y concentración observados. Se propone un esquema de reacción para la oxidación de OX a AF en el catalizador industrial.

En el capitulo 5 se presenta un estudio tebrico sobre el comportamiento del reactor industrial cuando se opera en estado transitorio. Se evalúa un modelo cinético que incorpora los fenómenos de desactivación reversibles e irreversibles en el catalizador $y$ se predice cualitativamente el comportamiento en estado transitorio del reactor.

En el capitulo 6 se presentan las conclusiones generales y algunas sugerencias para un trabajo a futuro. 


\section{Objetivos.}

\section{General y particulares}




\section{Objetivo general}

Estudiar el comportamiento de un reactor catalítico de lecho fijo en estado estacionario y transitorio para la reacción de oxidación parcial de o-xileno a anhidrido ftálico.

\section{Obietivos particulares}

1. Realizar el estudio hidrodinámico del reactor con el propósito de encontrar un modelo que permita predecir con la menor incertidumbre los campos de velocidad que son utilizados en el modelamiento del reactor con $y$ sin reacción quimica.

2. Estudiar teórica y experimentalmente los procesos de transporte de calor sin reacción, y así estimar los parámetros de transporte de calor, $h_{w} y k_{e f}$, que se usan en el modelamiento del reactor.

3. Estudiar el comportamiento del reactor en el estado estacionario mostrando el efecto de la desactivación del catalizador y el transporte de momento, calor y masa en la predicción de los perfiles de temperatura y concentración.

4. Predecir el comportamiento en el estado transitorio del reactor, mostrando el efecto de la desactivación del catalizador en la predicción cualitativa de su comportamiento. 
Capitulo 2.

Hidrodinámica

Capítulo 2.

Hidrodinámica 


\section{Eidrodinimica}

Algunos de lo trabajos [1-9] sobre el modelamiento de un reactor de lecho empacado, para reacciones altamente exotérmicas, se ha llevado a cabo con el objetivo de predecir los perfiles de concentración y temperatura experimentales. Lo que ha permitido tener un mejor entendimiento sobre el comportamiento de estos reactores. La mayoría de estos estudios ha considerado que la velocidad del fluido es uniforme a 10 largo del radio del lecho empacado. No obstante, en los últimos años se ha visto la necesidad de incluir el modelo hidrodinámico en el modelo del reactor, lo que parece ser esencial para predecir el comportamiento observado en reacciones altamente exotérmicas [14,27,3745]. Por ello este capitulo está dirigido ha estudiar algunos aspectos hidrodinámicos en el reactor de oxidación parcial de OX a AF.

En este capítulo se evalúan diferentes modelos hidrodinámicos que han sido utilizados de manera fundamental en la predicción de los perfiles de velocidad en un lecho empacado con una relación de $d_{k} / d_{p}$ menor a 10 . Estos modelos acoplan las ecuaciones de Navier-Stokes con los términos de Darcy y Forchheimer considerando el medio como pseudo-continuo y utilizando una ecuación empírica para predecir los perfiles de fracción vacía en el lecho. La evaluación de estos modelos permitio sugerir una forma de predecir con la menor incertidumbre los campos de velocidad en dos lechos empacados, uno a nivel laboratorio y otro industrial, que manejan una relación de $d_{c} / d_{p}$ similar, alrededor de 3 , pero diferente longitud.

En la sección 2.1 se presentan los antecedentes sobre estudios hidrodinámicos en lechos empacados que manejan una relación de $d_{c} / d_{p}$ menor a 10. En la sección 2.2 se muestran las características geométricas de los lechos empacados de los que se quieren conocer los perfiles de velocidad, donde se presta principal atención a los perfiles de fracción vacía y caída de presión que se tienen en estos sistemas. En la sección 2.3 se muestra el modelo hidrodinámico que se utiliza en este estudio para predecir los carmos de velocidad. En la 
sección 2.4 se presenta una comparación entre los perfiles de velocidad obtenidos con el modelo hidrodinámico que se utiliza en este estudio y aquéllos reportados en la literatura. En la sección 2.5 se evaluan las diferentes derivaciones del modelo hidrodinámico presentado en la sección 2.3. En la sección 2.6 se presentan las conclusiones y perspectivas sobre el estudio hidrodinámico en un lecho empacado con una baja relación de $d_{\varepsilon} / d_{p}$.

\subsection{Hidrodinámica en un lecho empacado}

Henri Darcy en 1856 [69] realiz6 uno de los primeros estudios hidrodinámicos en un lecho empacado, donde propone un modelo para predecir la velocidad media en éste. En su trabajo experimental maneja bajas velocidades de flujo y utiliza como empaque particulas pequeñas de tamaño uniforme. Bajo esta situación encontro que las resistencias dominantes al paso del flujo se deben a los esfuerzos de corte viscosos en las superficies sólidas. Estas resistencias viscosas se consideran en su modelo con la viscosidad del fluido y la permeabilidad del lecho empacado. Este modelo esta dado por la siguiente ecuación:

$v=-\frac{k}{\mu_{\varepsilon}}\left[\frac{d p}{d z}-\rho_{\varepsilon} g\right]$

La permeabilidad $(k)$ es una medida cuantitativa de la facilidad con la cual un fluido se transporta a través del lecho empacado, la cual depende de la naturaleza intrínseca del sblido. En la actualidad existen muchas relaciones que definen a la permeabilidad en términos de la fracción vacía, sin embargo, su definición matemática sigue teniendo incertidumbre hoy en día (ver Apéndice A).

La ley de Darcy tiene varias desventajas ya que los efectos inerciales

se han ignorado y las perdidas por fricción se han balanceado con la calda de presión y las fuerzas volumetricas, por lo que esta ley es 
valida a bajas velocidades de flujo. Además esta ley no requiere de la condición de velocidad en las fronteras del lecho empacado, haciendo que dicha ley sea valida lejos de estas y aplicable solamente en sistemas con una relación de $d_{k} / d_{p}$ mucho mayor a 10. Actualmente se reconoce que los efectos no Darcyanos son significantes en ciertas aplicaciones. Principalmente al utilizar altas velocidades de alimentación y sistemas con bajas relaciones de $d_{\varepsilon} / d_{p}$. La combinación de estas dos caracteristicas hace que los efectos inerciales en la interfase sరlido-fluido tengan que ser considerados en el modelo hidrodinámico. Esto ha obligado a que diferentes modelos semiheuristicos sean propuestos. Los cuales pueden incluir o no al término sugerido por Darcy, asi como a otros términos como los reportados por Ergun, Irmay, Beck, Forchheimer, Brinkman $[60,70,73,74]$ que consideran los fenómenos viscosos e inerciales presentes en el lecho. Asimismo diversos estudios [71,75] han mostrado que la permeabilidad $y$ el número de Darcy $\left(R_{t}^{2} / k\right)$, en un lecho empacado con una relación de $d_{c} / d_{p}$ mayor a 10 , se relaciona con el modelo hidrodinámico que puede ser utilizado para predecir los campos de velocidad. Para Da $<10^{-8}$ la ecuación de Darcy es adecuada; $5110^{-8}<\mathrm{Da}$ < $10^{-4}$ se debe incorporar la extensión de Brinkman; para $\mathrm{Da}>10^{-4}$ es indispensable incluir los efectos inerciales.

Se sabe que la representación de los perfiles de velocidad en un lecho empacado es difícil, debido a la discontinuidad de éste y a la variación de la fracción vacía en el lecho. Esto se agrava aún más por la falta de mediciones confiables de.velocidad dentro del lecho empacado. En principio, los perfiles de velocidad pueden ser descritos para cualquier geometría con las ecuaciones de Navier-Stokes. Sin embargo, la solución numérica de estas ecuaciones para un lecho empacado mediante códigos de CFD requiere de tiempos de cálculo muy altos $y$ actualmente esto ha sido aplicado s6lo a lechos empacados con una longitud pequeña y fracciones vacías grandes $[39,40,65,68]$. Otros investigadores han estudiado la hidrodinámica en lechos empacados de mayor longitud, utilizando los métodos de Lattice Boltzmann [58], sin embargo, se tienen inestabilidades numéricas y los tiempos de cálculo 
son muy altos. Debido a estas restricciones, es necesario llevar a cabo ciertas suposiciones razonables, las cuales permitan los mejores resultados con un costo computacional mínimo. Una aproximación en esta dirección es el uso del concepto de pseudo-continuo $[30,37,43-44,52-$ 56,60-63,66,69-76]. Bajo esta consideración, los campos de velocidad se describen por las ecuaciones modificadas de Navier-stokes. Normalmente se utilizan expresiones empiricas para describir los perfiles de fracción vacía. Las interacciones s6lido-fluido se consideran con términos adicionales en base a mediciones de calda de presión utilizando normalmente la ecuación de Ergun [73]. Esta forma de describir los perfiles de velocidad se conoce también como "los métodos de ingeniería aproximados", basado en los trabajos de "Darcy (1856) [69], Forchheimer [74], Brinkman (1947) [70], y Ergun (1952) [73], entre otros. Estos métodos se han utilizado por más de 70 años para predecir los perfiles de velocidad observados en un lecho empacado, sin embargo, la velocidad en las zonas de alta fracción vacía cerca de la pared $(\varepsilon-1)$ normalmente se sobreestima, por lo que se ha hecho uso de un parámetro empirico conocido como la viscosidad efectiva [70], que algunos autores usan como parámetro de ajuste para predecir las mediciones experimentales de velocidad $[54,56,63,70]$. Este parámetro genera gran incertidumbre, ya que se supone que es una función (no conocida) de la fracción vacía y del número de Reynolds, que se puede estimar a partir de estas mediciones. En los siguientes puntos se presenta un resumen sobre algunos trabajos que utilizan estos métodos de ingeniería aproximados para predecir los campos de velocidad en un lecho empacado que presenta un comportamiento no Darcyano.

Delmas y Froment [85] estudiaron el efecto del fenómeno hidrodinámico en la predicción de los perfiles de temperatura y concentración en un reactor de lecho fijo con una relación de $d_{k} / d_{p}$ igual a 6.25 , utilizado en la oxidación parcial de OX a AF. Este reactor fue operado a un $\mathbf{R e}_{\mathrm{p}}$ alrededor de 500. El modelo hidrodinámico que se utilizo es el siguiente: 


$$
\begin{aligned}
-\nabla p= & \nabla \cdot\left(\mu_{\varepsilon} \nabla v\right)+0.20\left[1+3.55\left(\frac{1-\varepsilon}{\varepsilon^{3}}\right)\right] \frac{p_{\varepsilon} v^{2}}{d_{\varepsilon}} \\
& +7.53\left(\frac{\varepsilon}{1-\varepsilon}\right)\left[1+4\left(\frac{1-\varepsilon}{\varepsilon^{3}}\right)\right] \frac{\mu v}{d_{\varepsilon}^{2}}
\end{aligned}
$$

El modelo considera la componente de la velocidad axial como función de la posición radial $y$ se resuelve en términos de la velocidad superficial. Asimismo, el término inercial que proviene de la ecuación de Navier-Stokes (termino de convección) no se considera, el cual parece ser clave para predecir los perfiles de velocidad cuando se trabaja con una velocidad de alimentación alta y un sistema que maneja una relación de $d_{\varepsilon} / d_{p}$ menor a 10. Kufner $y$ Hofmann [44] realizaron un estudio hidrodinámico en un reactor tubular utilizando un catalizador cilindrico, donde la relación de $d_{e} / d_{p}$ fue alrededor de 6 y el rango de $\mathrm{Re}_{\mathrm{p}}$ se encontró entre 500 y 2000 . Para predecir los campos de velocidad utilizaron el mismo modelo hidrodinámico que Delmas y Froment, por lo que se tienen las mismas incertidumbres en la predicción de los campos de velocidad. El modelo predice adecuadamente las observaciones de velocidad que se tuvieron en el centro del lecho pero no en la región cercana a la pared. Ambos modelos, así como los que se muestran más adelante, utilizan una ecuación empirica para predecir los perfiles de fracción vacía a lo largo del radio del reactor (ver Apéndice B).

Daszkowski y Eigenberger [61] estudiaron el efecto del fenómeno hidrodinámico en la predicción de los perfiles de concentración $y$ temperatura en un reactor de lecho fijo para la oxidación parcial de propeno utilizando un catalizador de paladio, donde la relación de $d_{t} / d_{p}$ fue de 5.1 y el $R e_{p}$ alrededor de 600. El modelo hidrodinámico que utilizaron se propone en términos de la velocidad intersticial y se resuelve en conjunto con los modelos seudo-homogéneos de masa y calor que describen al reactor. Dicho modelo esta dado por la siguiente ecuación: 
$\rho_{\varepsilon} u \cdot \nabla u=-\nabla p+\mu_{q} \nabla^{2} u-\frac{\mu_{f}}{k} \varepsilon u-\frac{\rho_{f}}{k_{2}(\varepsilon) d_{p}} \varepsilon^{2} u^{2}$

EL modelo es llamado la ecuación de Brinkman extendida, el cual fue propuesto inicialmente por vortmeyer et al. [104]. La permeabilidad k y $k_{2}$ son la mismas que se tienen en la ecuación de Ergun (ver Ecuaciones A.3 y A.4 en el Apéndice A). En este modelo se considera que las componentes de velocidad axial y radial son función de las coordenadas axial y radial del reactor. Se encontró que los perfiles de la velocidad radial son importantes unicamente en la primera capa de particulas de catalizador, ya que despues de ésta su valor es prácticamente cero. Asi también, después de esta primera capa de catalizador los perfiles de la velocidad axial, en la dirección radial, son iguales en cualquier posición axial del reactor. Si bien los resultados hidrodinámicos concuerdan cualitativamente con los resultados experimentales, las velocidades máximas locales se relacionaron con valores altos de la fracción vacía teniendo una velocidad mayor cerca de la pared. No obstante, el modelo hidrodinámico presenta las siguientes inconsistencias:

1. El segundo término del lado derecho de la Ecuación (2.3) no es realmente la extensión de Brinkman ya que no considera una viscosidad efectiva. Éste es el término viscoso que viene de la ecuación de Navier-Stokes.

2. Al utilizar la viscosidad dinámica del fluido y no la viscosidad efectiva en un sistema que maneja una baja relación de $d_{t} / d_{p}$ se tenían que haber re-estimado las constantes de la ecuación de Ergun ( $\alpha$ y $\beta$; ver ecuaciones A.3 y A.4 en el Apéndice A) para considerar adecuadamente las resistencias viscosas.e inerciales presentes en el reactor. No obstante, Daszkowski y Eigenberger [61] utilizaron las constantes reportadas por Ergun $(\alpha=150$ y $\beta=1.75)$. 
3. Si bien Daszkowski y Eigenberger [61] utilizaron un modelo hidrodinámico en términos de la velocidad intersticial, la ecuación de Navier Stokes que se acopla a la ecuación de Ergun no considera la variación de la fracción vacía en función de la posición axial y radial del lecho, lo cual aparentaria tener impacto en la predicción de los perfiles de velocidad.

Foumeny y Ma [62] estudiaron el comportamiento hidrodinámico de un fluido compresible en un lecho empacado bajo condiciones noisotérmicas. El sistema de estudio fue un lecho empacado que manejo relaciones de $d_{t} / d_{p}$ entre 5-20 y números de Reynolds de particula (Rep) entre 100-1000. El modelo hidrodinámico utilizado fue la ecuación de Forchheimer-Brinkman-Ergun, la cual es:

Ecuación de continuidad:

$\varepsilon \frac{\partial \rho_{f}}{\partial t}+\nabla\left(\rho_{t} v\right)=0$

Ecuación de movimiento:

$\frac{\rho_{f}}{\varepsilon} \frac{\partial v}{\partial t}=-\nabla p+\nabla \cdot\left(\frac{\mu_{f}}{\varepsilon} \nabla v\right)-\frac{\mu_{e}}{k} v-\frac{\rho_{f}}{k_{2}(\varepsilon) d_{p}} v^{2}+\varphi$

El vector $\varphi$ considera las fuerzas viscosas para un fluido compresible y son adicionales al segundo término del lado derecho; sin embargo, se encontró que este término es insignificante con respecto al término viscoso de Ergun. Este trabajo muestra el efecto de la temperatura en los perfiles de velocidad locales. Los resultados numéricos muestran diferencias hasta de $20 \%$ en los perfiles de velocidad, cuando se comparan los campos de velocidad de un fluido compresible con los de uno incompresible. Sin embargo, el modelo hidrodinámico utilizado por Foumeny y Ma [62] no consideró la funcionalidad de la fracción vacía dentro de los esfuerzos de corte viscosos en la ecuación de NavierStokes. Asimismo, los efectos inerciales debidos a Navier-stokes se omiten del modelo hidrodinámico. 
Papageorgiuou y Froment [30] simulan el comportamiento de un reactor de lecho fijo para la oxidación parcial de o-xileno a anhidrido ftalico utilizando un catalizador de $\mathrm{V}_{2} \mathrm{O}_{3} / \mathrm{TiO} \mathrm{O}_{2}$. La relación de $d_{e} / d_{p}$ es 5.8 , 10 que generó apreciables perfiles de fracción vacia en el interior de reactor. Para predecir los perfiles de velocidad se utilizo la siguiente ecuación:

$\rho_{\mathrm{f}} v \cdot \nabla v=-\nabla p+\mu_{f}\left(\nabla^{2} v\right)-\frac{\mu_{\mathrm{f}}}{k} v-\frac{\rho_{\mathrm{f}}}{k_{2}(\varepsilon) \mathrm{d}_{\mathrm{p}}} v^{2}$

El modelo considera las componentes de la velocidad axial y radial como función de la posición radial del reactor. Con la ecuación de Ergun se consideraron las interacciones entre el solido y el fluido. Estos autores llegan a resultados semejantes a los de Daszkowski y Eigenberger, donde el modelo muestra las mismas limitaciones que los modelos expuestos anteriormente.

Bey y Eigenberger [63] predijeron los campos de velocidad en un lecho empacado que manejó relaciones de diámetro de tubo a partícula entre 3.3 Y 11, para un rango de $\operatorname{Re}_{p}$ entre 50 y 1000. La hidrodinámica de este lecho se estudio utilizando diferentes geometrias como empaque: esferas, anillos y cilindros. Para predecir los campos de velocidad se utilizo. la siguiente ecuacion:

$\nabla_{p}=\frac{\tilde{\mu}}{\varepsilon} \nabla \cdot(\varepsilon \nabla u)-\varepsilon \frac{\mu_{f}}{k} u-\varepsilon^{2} \frac{\rho_{\varepsilon}}{k_{2}(\varepsilon) d_{p}} u^{2}$

Este modelo considera unicamente la componente de la velocidad axial en función de la posición radial, y omite los efectos inerciales y axiales viscosos de la ecuación de Navier-Stokes. Además de utilizar los mismos valores de las constantes de Ergun, en la descripción de los perfiles de velocidad se considera la viscosidad efectiva en el término viscoso de Navier-stokes, la cual permite ajustar sus resultados experimentales. 
Giese et al. [56] observaron experimentalmente, utilizando un equipo de rayos láser, los perfiles de velocidad de una mezcla liquida en un lecho empacado con esferas deformadas, cilindros y anillos raschig. La relación de $d_{t} / d_{p}$ fue alrededor de 9.3 y el rango de $R_{p}$ utilizado estuvo entre 4 y 530. Ellos utilizaron una derivación de la Ecuación (2.5) para predecir los perfiles de velocidad observados en el lecho, la cual consideró únicamente la componente superficial de velocidad axial como función del radio $y$ el termino convectivo debido a NavierStokes fue despreciado. El modelo que emplean se resolvió en términos de la velocidad superficial y se usa una viscosidad efectiva en el término viscoso de Brinkman para ajustar los resultados experimentales.

Winterberg y Tsotsas [54] estudiaron el impacto de la fracción vacia y los efectos de fricción en la predicción de la caída de presión en un lecho empacado. Para esto utilizaron sistemas con diversas relaciones de $d_{c} / d_{p}(4-40)$ y $R e_{p}$ entre 1 y 1000. El modelo hidrodinámico que utilizaron fue la ecuación extendida de Brinkman, es decir, la misma que utilizó Giese et al. [56]. Winterberg et al. [66] predicen los perfiles de concentración y temperatura en un reactor con esferas, cuya relación $d_{c} / d_{p}$ fue igual a 8 y un $R e_{p}$ alrededor de 600 ; donde se considera el fenómeno hidrodinámico. El modelo hidrodinámico es el mismo que el utilizado por Giese et al. [56]. Por lo que las inconsistencias que presenta este modelo hidrodinámico son las mimas que las comentadas en párrafos anteriores. Otros estudios $[43,55,67,72,109,121,138]$, no comentados aqui, han utilizado los mismos modelos hidrodinámicos que los ya mencionados para predecir los campos de velocidad en sistemas con un comportamiento no Darcyano, lo que hace dificil el estudio de esta clase de lechos.

Eisfeld y Schnitzlein [60] realizaron uno de los estudios más formales para describir los perfiles de velocidad en un lecho empacado que presenta una baja relación de diámetro de tubo a particula. El modelo propuesto se resuelve en términos de la velocidad intersticial y esta dado por las siguientes ecuaciones: 
Continuidad:

$\frac{\partial \varepsilon \rho_{\varepsilon}}{\partial t}+\nabla\left(\rho_{t} \varepsilon u\right)=0$

Velocidad:

$\frac{\partial \rho_{\varepsilon} u}{\partial t}+\nabla \cdot\left(\varepsilon \rho_{\varepsilon} u u\right)=-\nabla(\varepsilon p)+\nabla \cdot(\varepsilon \tau)-\mathbf{R}_{g-\bullet}$

El último término del lado derecho considera las interacciones solidofluido debido a la presencia del medio solido (ver sección 2.2). Estas interacciones se consideran utilizando una correlación de calda de presión reportada y evaluada por Eisfeld [80]. La cual es la ecuación de Ergun modificada. Este modelo hidrodinámico se valida al predecir los perfiles de velocidad experimentales en lechos empacados que manejan una relación de $d_{c} / d_{p}$ menor a 10 y velocidades de alimentación altas. Las predicciones fueron relativamente adecuadas, sin embargo, encontraron algunos problemas para predecir las velocidades locales en regiones de alta fracción vacía.

En la mayoría de los estudios hidrodinámicos en un lecho empacado con una baja relación de $d_{\varepsilon} / d_{p}$ se utilizan modelos hidrodinámicos que predicen razonablemente bien el comportamiento observado de los campos de velocidad, sin embargo, quedan muchas preguntas sobre las suposiciones planteadas en el uso de cada uno de estos modelos, como por ejemplo: ¿es adecuado el uso de la viscosidad efectiva?, ¿el modelo se debe resolver en términos de la velocidad superficial o intersticial?, ¿la magnitud de los términos inercial y viscoso, considerados con la ecuación de Ergun, son característicos de cada lecho?, ¿que términos son significantes y necesarios dentro del modelo hidrodinámico para predecir los perfiles de velocidad adecuadamente?. La necesidad de conocer un modelo hidrodinámico que prediga con Ia menor incertidumbre los perfiles de velocidad en el reactor estudiado $y$ el tratar de responder estas preguntas hace de este estudio hidrodinámico un trabajo relevante. Para conseguir este objetivo se comparan diferentes modelos hidrodinámicos reportados en la literatura, 
asi como diferentes derivaciones de un modelo hidrodinámico general, donde se pone principal atención a la forma en que se consideran las interacciones entre el sólido y el fluido.

\subsection{Modelo hidrodinámico}

El sistema de estudio contiene dos fases: un sólido y un gas. El modelo utilizado para predecir los campos de velocidad considera los siguientes aspectos:

1. Se describe el movimiento del fluido desde un punto de vista de un medio continuo aunque se considera al sistema como un medio pseudocontinuo debido a la presencia del medio sólido.

2. El fluido es newtoniano e incompresible.

3. La viscosidad y densidad son constantes.

El modelo hidrodinamico que considera los tres puntos anteriores en términos de la velocidad intersticial, que acopla la ecuación de Navier-Stokes con los términos que describen a la interacción entre el fluido y el sólido, es el siguiente $[49,51,72]$ :

$\frac{\partial}{\partial t}\left(\rho_{t} \varepsilon u\right)+\rho_{\varepsilon} \varepsilon u \cdot(\nabla \varepsilon u)=-\nabla \varepsilon p+\mu_{i} \nabla^{2} \varepsilon u+\rho_{z} \mathbf{g}-\mathbf{R}_{g-s}$

Donde :

$\left[\frac{\partial}{\partial t}\left(\rho_{\varepsilon} \varepsilon u\right)+\rho_{\varepsilon} \varepsilon u \cdot(\nabla \varepsilon u)\right]:$ Fuerzas inerciales provenientes de la ecuación de Navier-Stokes.

$\nabla \varepsilon p:$ Gradiente de presion

$\mu_{\mathrm{f}} \nabla^{2} \varepsilon u$ : Esfuerzos de corte viscosos, también llamado termino viscoso de Brinkman cuando se utiliza una viscosidad efectiva. 
$\mathbf{B}_{\text {g-s: }}$ Interacciones entre el sólido y el fluido. Las cuales consideran las resistencias viscosas e inerciales al paso del fluido debidas a la presencia del solido.

\subsubsection{Interacciones entre el sólido y fluido}

Las interacciones entre el solido y el fluido, dependen de la magnitud del número de Reynolds $y$ de la superficie solida en el lecho. El

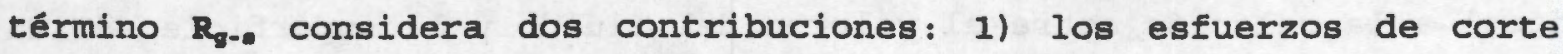
viscosos $R e_{p}<10$ y 2 l los esfuerzos inerciales $R e_{p}>10$. Asi, las fuerzas debidas a la presencia del sólido se expresan como la suma:

$\mathbf{R}_{\mathrm{ge}}=\mathbf{R}_{\mathrm{ge}}{ }^{(\mathrm{BF})}+\mathbf{R}_{\mathrm{ge}}{ }^{(\mathrm{AF})}$

Normalmente la contribución a los esfuerzos de corte viscosos ha sido expresada por el término de Darcy (1856) [69], de la siguiente manera:

$R_{g=}^{(B F)}=\frac{\mu_{\varepsilon}}{k(\varepsilon) d_{p}^{2}} \varepsilon u$

Por otro lado, para el caso de flujos altos de alimentación $\left(R e_{p}>10\right)$, los efectos inerciales debidos a la presencia del sólido son más importantes, por lo que se requiere una expresión que describa este comportamiento, y que de alguna manera tome en cuenta el nivel de la turbulencia presente. Haciendo una analogía con las expresiones que se emplean para predeçir la caída de presión en lechos empacados, se tiene que :

$R_{g s}{ }^{(A F)}=\frac{\rho_{\varepsilon}}{k_{2}(\varepsilon) d_{p}} \varepsilon^{2} u^{2}$

Este término tiene la forma del segundo término de la expresión propuesta por Forchheimer [74]. 
El diámetro de particula es un parámetro de suma importancia en el estudio de lechos empacados, ya que para sistemas en donde, para un mismo radio de tubo, se varía el diámetro de partícula, la caída de presión ocasionada es diferente. Esto se debe a la diferencia en la distribución de la fracción vacia en el lecho para cada caso. Este problema ha Ilevado a diferentes autores a proponer el concepto del radio hidrálico [51,52], para considerar los efectos debidos a la distribución de la superficie sólida, y comparar los resultados obtenidos con diferentes empaques y geometrias. El radio hidráulico es igual a la relación entre el volumen del fluido y la superficie solida presente. El cual se define en términos de la fracción vacía. Así, la ecuación que representa a las interacciones sólido-fluido para un lecho empacado, en términos de un radio hidráulico, es:

$R_{g=}=\frac{\mu_{t}}{k(\varepsilon) d_{p}^{2}} u+\frac{p_{f}}{k_{2}(\varepsilon) d_{p}} u^{2}$

Los diversos modelos utilizados en la literatura, para predecir los campos de velocidad, usan diferentes expresiones para las variables $k(\varepsilon)$ y $k_{2}(\varepsilon)$. Estas expresiones tienen una relación directa con las ecuaciones usadas para calcular la caída de presión en lechos empacados y que normalmente han sido utilizadas en sistemas con bajas relaciones de $d_{t} / d_{p}$. Las siguientes ecuaciones muestran la funcionalidad de $k(\varepsilon)$ y $k_{2}(\varepsilon)$ con la fracción vacia, según Ergun [73]:

$$
\begin{aligned}
& k(\varepsilon)=-\frac{1}{150\left(\frac{(1-\varepsilon)^{2}}{\varepsilon^{3}}\right)} ; \text { Para } R e_{p}<10 \\
& k_{2}(\varepsilon)=-\frac{1}{1.75\left(\frac{1-\varepsilon}{\varepsilon^{3}}\right)} ; \text { Para } R e_{p}>10
\end{aligned}
$$


En el Apéndice A se presentan otras ecuaciones utilizadas en la literatura para definir. $k(\varepsilon)$ y $k_{2}(\varepsilon)$. SI $R_{g-s}$ depende fuertemente de la fracción vacía, de las ecuaciones $(2.14-2.16)$ y las ecuaciones presentadas en el Apéndice A se puede observar que si no existe la presencia de un empaque, la fracción vacía $\varepsilon$ es 1 y $R_{g-s}$ es cero, por 10 que se recuperan las ecuaciones de Navier-Stokes. Por otro lado, si el sistema tienen una alta relación de $d_{c} / d_{p}$ (p.e. mayor a 10), y $R_{g-s}$ utiliza los términos viscoso e Inercial de Ergun [73], se recuperan las expresiones para determinar la caida de presión. Para un sistema con una baja relación $d_{t} / d_{p}$, la fracción vacía varía significativamente a lo largo de los ejes axial y radial del lecho empacado, por 10 que debe considerarse su variación axial y radial.

\subsubsection{Perfiles de fracción vacía}

En la presente sección se hace un análisis sobre los perfiles de fracción vacía que se tienen en un lecho empacado con una relación de $d_{\varepsilon} / d_{p}$ menor a 10 . Estos perfiles de fracción vacía se relacionan con distintos procesos hidrodinámicos debidos a la presencia del sólido. Uno de éstos se relaciona con el incremento de la velocidad de flujo en la vecindad del sólido teniendo un espesor de la capa límite despreciable alrededor de las superficies sరlidas. Otro proceso es la importancia de los efectos de fricción en las superficies sólidas, a flujos bajos, en donde el espesor de la capa limite alrededor de las superficies s6lidas es apreciable. En este sentido, la magnitud del flujo de alimentación $y$ de los perfiles de fracción vacía juega un papel importante en la hidrodinámica en el interior de un lecho empacado.

La fracción vacía juega un papel importante en la calda de presion y en la permeabilidad en un lecho empacado, principalmente en un lecho con una relación de $d_{c} / d_{p}$ menor a 10 [47-63]. Sin embargo, aún existe incertidumbre en la descripción de los perfiles de fracción vacía y de 
las distintas configuraciones que tiene el empaque dentro del lecho. Debido a esto, es importante cuantificar los perfiles de fracción vacia en estos sistemas, por su efecto en los campos de velocidad, y por consecuencia en los fenómenos de transporte de calor y masa en reactores de lecho empacado $[41-45]$.

Actualmente se emplean varios métodos experimentales para cuantificar los perfiles de fracción vacía [59]. El método más común consiste en colocar resina epóxica en el lecho empacado para mantener a las particulas dentro de una posición fija y medir la fracción vacía, la cual se calcula por un balance de materia [51,59]. Existen otras técnicas para medir los perfiles de fracción vacía en un lecho empacados, sin embargo, no se tratan en este trabajo.

Las observaciones experimentales deben expresarse mediante una relación matemática para emplearse dentro de las ecuaciones que describen el transporte de momento, calor y masa en un lecho empacado. Los modelos que se usan para describir a los perflles de fracción vacía en lechos empacados son de dos tipos. Los primeros usan las relaciones empiricas derivadas de mediciones experimentales en lechos empacados con diferente relación $d_{c} / d_{p}[46-51,55,56,59]$. Los segundos, son modelos matemáticos que describen a esferas empacadas de acuerdo a un cierto arreglo geométrico (rómbico, hexagonal, de celdas, etc.)[51] y los derivados de modelos probabilísticos de tipo Monte Carlo, etc [51]. Todos estos métodos sufren de las mismas limitaciones $y$ han sido relativamente exitosos en la predicción de mediciones experimentales.

En este estudio se eligio la expresión empirica de de-klerk [59], la cual se ha caracterizado por describir con relativo éxito a una gran cantidad de perfiles experimentales fracción vacía. Esta correlación describe los perfiles de fracción vacía en dos regiones del lecho empacado, cerca de la pared y en el centro: 
En la región cercana a la pared del lecho:

$$
\varepsilon(r)=2.14\left(\frac{\left(R_{T}-r\right)}{d_{p}}\right)^{2}-2.53\left(\frac{\left(R_{T}-r\right)}{d_{p}}\right)+1
$$

Para $\frac{\left(R_{T}-r\right)}{d_{p}}<0.637$

En el centro del lecho:

$$
\begin{array}{r}
\varepsilon(r)=\varepsilon_{-}+0.29 \exp \left[-0.6 *\left(\frac{\left(R_{T}-r\right)}{d_{p}}\right)\right] *\left(\cos \left(2.3 \pi\left[\frac{\left(R_{T}-r\right)}{d_{p}}-0.16\right]\right)\right. \\
+0.15 \exp \left(-0.9\left[\frac{\left(R_{T}-r\right)}{d_{p}}\right]\right)
\end{array}
$$

Para $\frac{\left(R_{T}-r\right)}{d_{p}}>0.637$

En el Apéndice B se presentan algunas correlaciones que han sido utilizadas para predecir los perfiles fracción vacía en un lecho empacado con una baja relación de $d_{t} / d_{p}$. Dada la naturaleza empirica de estas ecuaciones, que solo describen la variación radial de la fracción vacia, no es posible describir los perfiles tridimensionales de fracción existentes en lechos empacados. Esto tendrá consecuencias cuando los perfiles de fracción vacía se utilicen en la predicción de los perfiles de velocidad intersticial.

\subsection{Hidrodinámica del sistema de estudio: resultađos y discusión}

El estudio hidrodinámico se realizó en dos lechos empleando el mismo empaque, la misma relación de $d_{c} / d_{p} y$ diferente longitud. Las caracterfsticas geométricas esenciales de estos lechos para realizar el estudio hidrodinámico se presentan en la Tabla 2.1 . 
Tabla 2.1.

Datos geometricos y fisicos de los lechos:

de laboratorio $y$ a escala industrial

\begin{tabular}{lr}
\hline Propiedad & Magnitud \\
\hline Reactor & \\
Longitud del lecho industrial, m & 2.6 \\
Longitud del lecho de laboratorio, m & 0.41 \\
Diámetro, m & 0.025 \\
\hline Catalizador esferico no poroso & \\
\hline Diámetro, m & 0.0082 \\
Fracción vacla promedio del lecho & $0.48 *$ \\
Relacion $d_{c} / d_{p}$ & 3.125 \\
\hline
\end{tabular}

La Figura 2.1 muestra fotografías del aparente acomodo del catalizador de $\mathrm{V}_{2} \mathrm{O}_{3} / \mathrm{TiO}_{2}$ en el reactor. Éstas se tomaron al empacar el catalizador en un tubo de acrílico que tenía el mismo diámetro que el reactor estudiado a escala industrial. En estas fotografías se observan variaciones de la fracción vacía en las tres dimensiones. Asimismo, se observa, en la dirección radial (Fig. 2.1b), en varias posiciones tangenciales, que hay dos zonas de mayor fracción vacla, una cerca de la pared y otra a una distancia de un diámetro de partícula de ésta. En la direcciones axial y tangencial (Figuras 2.1a y 2.1c) se presenta una variación periodica de la fracción vacía, la cual no ha sido incluida en ninguna de las correlaciones reportadas a la fecha, y aparenta tener efecto en la descripción de los campos de velocidad (ver Apéndices C y D) .

En la Tabla 2.2 se muestran las condiciones experimentales utilizadas en el estudio hidrodinámico realizado en el reactor de oxidación parcial de $O X$ a $A F$. Las corridas 1 a 3 emplean flujos que normalmente se utilizan en reactores a escala laboratorio, mientras que los datos en 4 a 7 se utilizan en reactores de escala piloto e industrial. En ambos lechos, de laboratorio y escala industrial, no se midieron los perfiles de velocidad en el interior del reactor. Para predecir los 
campos de velocidad se utilizaron datos experimentales de caída de presión tonados del trabajo de López Isunza en el reactor a escala industrial [14].

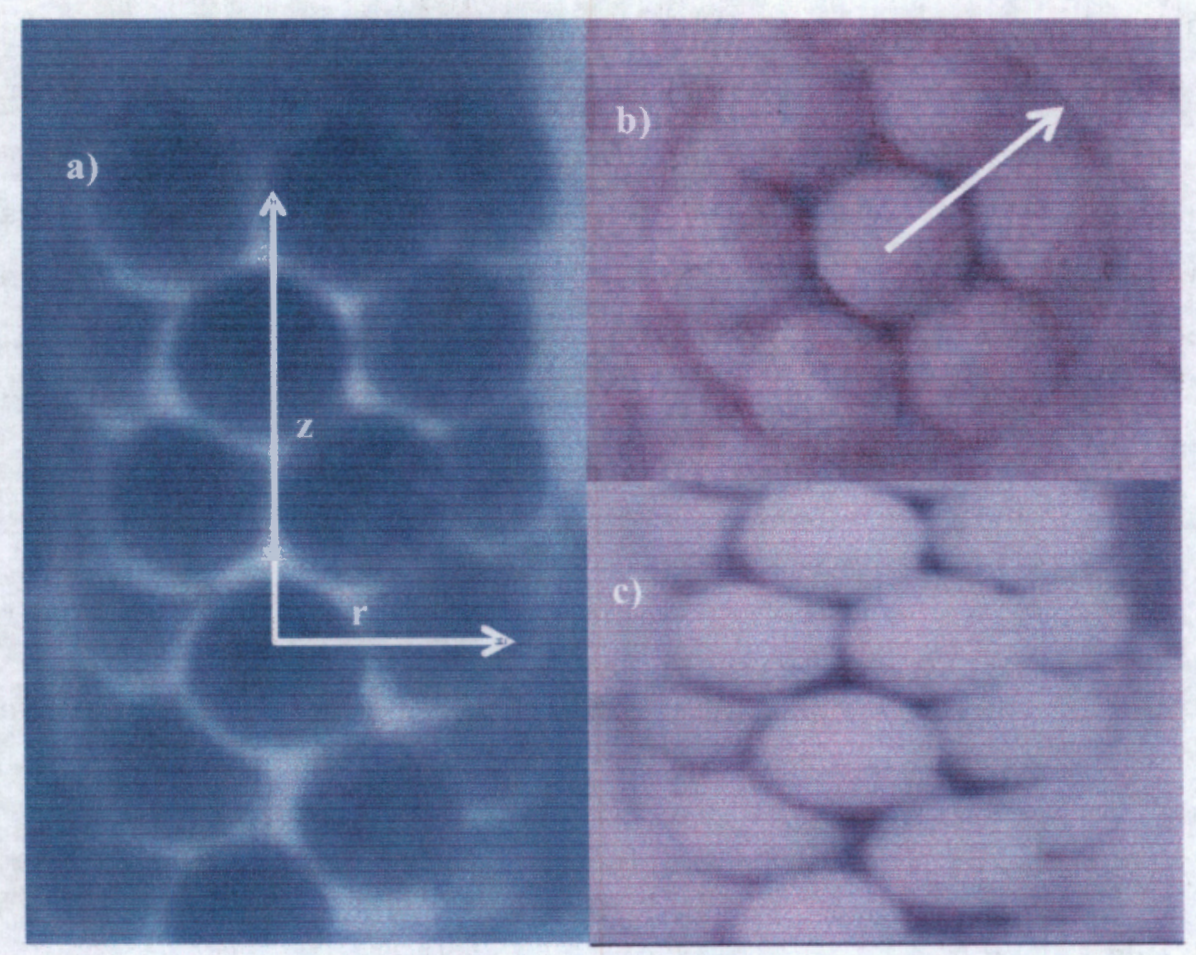

Figura 2.1. Fotografía que describe el arreglo de las esferas de catal:zador en el interior de un tubo de acrilico del mismo dímetro que el reactor industrial. a) Negativo de Ia vista lateral, b) vista superfor $y$ cl vista lateral

Tabla 2.2.

Condiciones de operación a las que se quieren los perfiles velocidad

\begin{tabular}{cccc}
\hline Corrida & $G_{8} \mathrm{~kg} \cdot \mathrm{m}^{-2} \cdot \mathrm{h}^{-1}$ & $\mathrm{Re}_{\mathrm{p}}$ & $\Delta \mathrm{P}_{8} \mathrm{~N} \cdot \mathrm{m}^{-2}[14]$ \\
\hline 1 & 720 & 25 & - \\
2 & 1800 & 60 & - \\
3 & 3960 & 160 & - \\
4 & 5274 & 315 & 9800 \\
5 & 7909 & 470 & 18500 \\
6 & 10548 & 630 & 29300 \\
7 & 13183 & 785 & 41000 \\
\hline
\end{tabular}


Para predecir los perfiles de velocidad se considera a un lecho empacado cuya fracción vacía varia sólo en la dirección radial. Si bien esto es una limitación, no es posible describir aquí la variación de la vacía en las tres dimensiones. El gas que se utiliza en la reacción de oxidación parcial es una mezcla de aire $99 \%$ mol y $1 \%$ mol 0-Xileno, por lo que la densidad y la viscosidad del aire se utilizan en la predicción de los perfiles de velocidad en el lecho, cuyos valores, evaluados a la temperatura de alimentación, se consideran constantes en todo el lecho. Lo anterior permite suponer que la permeabilidad es función únicamente de la fracción vacía. La velocidad axial y radial es función de las posiciones radial y axial en el lecho. El modelo adimensional, basado en estas suposiciones $y$ en aquéllas mencionadas en la sección 2.2 , está dada por:

Dirección axial, considerando despreciable el efecto de la gravedad:

$$
\begin{aligned}
& \frac{\partial \mathrm{U}_{z}}{\partial \tau}+\mathrm{R}_{\mathrm{es}} \varepsilon \mathrm{U}_{\mathrm{z}} \frac{\partial \mathrm{U}_{z}}{\partial \zeta}+\mathrm{R}_{\mathrm{e}} \varepsilon \mathrm{U}_{x} \frac{\partial \mathrm{U}_{z}}{\partial \xi}+\mathrm{R}_{\mathrm{e}} \mathrm{U}_{\mathrm{r}} \mathrm{U}_{\mathrm{z}} \frac{\partial \varepsilon}{\partial \xi}= \\
& \psi_{\mathrm{z}}+\left(\frac{\partial^{2} \mathrm{U}_{z}}{\partial \xi^{2}}+\left(\frac{2}{\varepsilon} \frac{\partial \varepsilon}{\partial \xi}+\frac{1}{\varepsilon}\right) \frac{\partial \mathrm{U}_{z}}{\partial \xi}+\left(\frac{1}{\varepsilon \xi} \frac{\partial \varepsilon}{\partial \xi}+\frac{1}{\varepsilon} \frac{\partial^{2} \varepsilon}{\partial \xi^{2}}\right) \mathrm{U}_{\mathrm{z}}+\mathrm{RL} \frac{\partial^{2} \mathrm{U}_{\mathrm{z}}}{\partial \zeta^{2}}\right)-\frac{\mathrm{U}_{z}}{\mathrm{Da}}-\frac{\mathrm{U}_{z}^{2}}{\mathrm{Da}_{2}}
\end{aligned}
$$

Dirección Radial:

$$
\begin{aligned}
& \frac{\partial \mathrm{U}_{x}}{\partial \tau}+\mathrm{R}_{e s} \varepsilon \mathrm{U}_{z} \frac{\partial \mathrm{U}_{x}}{\partial \zeta}+\mathrm{R}_{\mathrm{e}} \varepsilon \mathrm{U}_{x} \frac{\partial \mathrm{U}_{x}}{\partial \xi}+\mathrm{R}_{\mathrm{e}} \mathrm{U}_{x}^{2} \frac{\partial \varepsilon}{\partial \xi}= \\
& \Psi_{x}+\left(\frac{\partial^{2} \mathrm{U}_{x}}{\partial \xi^{2}}+\left(\frac{2}{\varepsilon} \frac{\partial \varepsilon}{\partial \xi}+\frac{1}{\varepsilon}\right) \frac{\partial \mathrm{U}_{x}}{\partial \xi}+\left(\frac{1}{\varepsilon \xi} \frac{\partial \varepsilon}{\partial \xi}+\frac{1}{\varepsilon} \frac{\partial^{2} \varepsilon}{\partial \xi^{2}}-\frac{1}{\xi^{2}}\right) U_{x}+\mathrm{R} \frac{\partial^{2} U_{x}}{\partial \zeta^{2}}\right)-\frac{\mathrm{U}_{x}}{\mathrm{Da}}-\frac{\mathrm{U}_{x}^{2}}{D \mathrm{a}_{2}}
\end{aligned}
$$

La condición inicial y de frontera que representa la situación física del sistema está dada por:

Tiempo:

$\tau=0$

$$
U_{z}=U_{z}(0, \xi, \zeta), \quad U_{x}=U_{x}(0, \xi, \zeta)
$$


Posición Axial:

$\zeta=0$

$$
\mathrm{U}_{\mathrm{z}}=\mathrm{U}_{\mathrm{in}}, . \mathrm{U}_{\mathrm{r}}=0
$$

$\zeta=1$

$$
\frac{\partial U_{z}}{\partial \zeta}=0, \frac{\partial u_{r}}{\partial \zeta}=0
$$

Poaición Radial:

$\xi=0$

$$
\frac{\partial U_{z}}{\partial \xi}=0, \frac{\partial U_{r}}{\partial \xi}=0
$$

$\xi=1$

$$
U_{z}=0, U_{x}=0
$$

Todos los modelos utilizados en este estudio son ecuaciones diferenciales parciales del tipo parabólico (EDP), en las cuales se discretizaron las coordenadas espaciales con el método de colocación ortogonal ( $\mathrm{CO}$, utilizando polinomios de Legendre [78]. Las ecuaciones resultantes constituyen un sistema de ecuaciones diferenciales ordinarias (EDO) que se resolviexon con el método de Runge-KuttaFehlberg [79].

Ia estimación de los parámetros, cuando se requirio, se llevo a cabo con el método de Levenberg-Marquadt [77]. Donde el intervalo de confidencia para todos lo casos fue de 95\%. El método de LevenbergMarquadt combina el método de Gauss (series de Taylor) y descenso más pronunciado dando como resultados la estimación de los parámetros $\theta$ que se desean conocer.

Para obtener el valor de los parámetros que se desean estimar en cada caso se usan los datos experimentales y el modelo correspondiente. Los parámetros se obtienen resolviendo el problema de mínimos cuadrados donde se desea minimizar la siguiente función.

$$
S(\theta)=\sum_{u=1}^{n}\left(Y_{u}-I\left(\theta, \xi_{u}\right)\right)^{2}
$$


La estimación de los parámetros $\theta$ se puede llevar a cabo cuando el modelo matemático es lineal o no con respecto a los parámetros desconocidos.

Las herramientas matemáticas utilizadas en este trabajo se pueden revisar con mayor detalle en las referencias $[38,75,77,79]$.

\subsubsection{Calda de presion}

El estudio de la calda de presión en lechos empacados es importante en el diseño de reactores. Ya que conocer la calda de presión en un lecho empacado es indispensable para predecir los perfiles de velocidad, con menor incertidumbre, en sistemas en donde no se tienen datos experimentales de velocidad. En la mayoría de los estudios hidrodinámicos, la obtención de los mejores resultados depende de la correlación de calda de presión que se utiliza, ya que ésta determina la magnitud de las resistencias viscosas e inerciales al paso del fluido.

La mayoría de los estudios sobre la caida de presión en lechos empacados $\{60,73,80-83]$ han encontrado que ésta depende del número de Reynolds de particula $\left(R e_{p}\right)$, de la fracción vacía promedio, y de la geometría de la particula y su distribución de tamaño. La magnitud de $\mathrm{Re}_{\mathrm{p}}$ define el régimen de flujo, es decir, un flujo laminar para $R e_{p}<10$, una transición entre flujos laminar $Y$ turbulento cuando $10<R e_{p}<300$ y un flujo turbulento para un $R e_{p}>300$ [80]. Como se mencionó la calda de presión es sensible a la fracción vacia, lo que se relaciona con las superficies sólidas presentes en el lecho. Para incorporar este efecto se usa el concepto de radio hidráulico $[60,75,80-83]$. La siguiente ecuación es la forma más general para representar a la caída de presión en lechos empacados en términos del radio hidráulico, para el régimen, laminar, transición y turbulento: 


$$
\frac{\Delta p}{L}=\frac{\alpha \mu_{q} v}{d_{p}^{2}} m(\bar{\varepsilon})+\frac{\beta \rho_{\varepsilon} v^{2}}{d_{p}} m_{2}(\bar{\varepsilon})
$$

Donde $m(\bar{\varepsilon})$ y $\mathrm{m}_{2}(\bar{\varepsilon})$ son los radios hidráulicos para régimen laminar, $y$ transición $y$ turbulento, respectivamente, $y$ :

$$
\begin{aligned}
& \frac{1}{\mathrm{k}(\bar{\varepsilon})}=\alpha \mathrm{m}(\bar{\varepsilon}) \\
& \frac{1}{\mathrm{k}_{2}(\bar{\varepsilon})}=\beta \mathrm{m}_{2}(\bar{\varepsilon})
\end{aligned}
$$

Los valores de $\alpha, m(\bar{\varepsilon}), \quad \beta$ y $m_{2}(\bar{\varepsilon})$ varian entre las diversas correlaciones y la geometría de la particula (ver términos en el Apéndice A). La correlación que se emplea con mayor frecuencia para ajustar los datos de calda de presión y predecir los perfiles de velocidad en lechos empacados es la propuesta por Ergun [73], en donde $\alpha=150$ y $\beta=1.75$ (Ecuación (2.27)). Sin embargo, esta ecuación no considera adecuadamente las resistencias al flujo del fluido en lechos empacados con una relación de diametro tubo a particula $\left(d_{c} / d_{p}\right)$ menor a 10 [80]. Debido a esto, diversos autores han propuesto diferentes correlaciones que consideren estos fenómenos.

En este estudio la Ecuación (2.26) se acoplo a la Ecuación de NavierStokes, teniendo la Ecuación (2.10), para predecir los perfiles de velocidad en el lecho empacado, donde las Ecuación (2.26) considera las interacciones entre el sólido $y$ el fluido. Para tener una adecuada predicción de los perfiles de velocidad, los parámetros $\alpha$ y $\beta$ fueron re-estimados utilizando los datos de calda de presión que se observaron en el reactor a escala industrial. Es decix, la contribucion de los esfuerzos viscosos, $\alpha$, los cuales dominan en un régimen de flujo laminar, y la contribución debida a los efectos inerciales, $\beta$, Ios cuales son importantes en un régimen de flujo de transición y turbulento, se estimaron al utilizar las ecuaciones (2.26)-(2.28) y los datos experimentales de calda de presión presentados en la Tabla 2.2. 
La estimación de $\alpha$ y $\beta$ se llevo a cabo por el algoritmo de LevenbergMarquadt [77] utilizando como valores iniciales para la estimación aquéllos reportados por Ergun [73]. AsI también, los datos experimentales de calda de presión, tomados del trabajo de Lobez-Isunza [14], se comparan con aquéllos obtenidos de correlaciones propuestas en la literatura $y$ utilizadas en los rangos de $d_{c} / d_{p}$ y números de Reynolds expuestos en la sección 2.1 (ver Apéndice A) $[44,58,73,74,80-83]$.

En la Figura 2.2a se presenta una comparación entre los datos experimentales de caída de presión a diferentes flujos de alimentación, los datos de caída de presión que se ajustan a la correlación de Ergun re-estimando los valores de $\alpha$ y $\beta$ lcuyos minimos globales son: $\alpha=1028$ y $\beta=1.15$ ) Y las predicciones de otras correlaciones utilizadas en la literatura para estudiar sistemas con una baja relación de $d_{t} / d_{p}$. Para valores de $\mathrm{Re}_{\mathrm{p}}$ mayores a 160, la caida de presión predicha por la mayoría de las correlaciones fue mayor que la ajustada con la ecuacion de Ergun habiendo estimado $\alpha$ y $\beta$. Al estimar las constantes $\alpha$ y $\beta$, las diferencias entre los datos experimentales y los valores ajustados de caída de presión son menores al 5\%. Mientras que, cuando se emplearon las otras correlaciones, las diferencias entre predicciones y datos experimentales varian entre 10 y 100\%, dependiendo del flujo de alimentación y de la correlación empleada. Lo anterior también puede observarse en el diagrama de paridad mostrado en la Figura 2.2b. En la Figura $2.3 \mathrm{~b}$ se muestra una comparación entre las predicciones de la calda de presión, para $\mathrm{Re}_{\mathrm{p}}$ de 25-160. Aquí se emplea correlación de caída de presión con los valores re-estimados de a y $\beta$, y las correlaciones que tienen el menor error en la predicción de la caída de presión a flujos altos: Leva [82], Macdonald et al. [82], Eisfeld et al. [80] y Freund et al. [58]. Los resultados que arrojan estas correlaciones varían en más de 50 en comparación con los resultados obtenidos con la Ecuación de Ergun modificada ( $\alpha=1028$ y $\beta=1.15)$. Estos resultados confirman los expuestos arriba, donde cada sistema presenta resistencias al flujo que dependen de la configuración geométrica y propiedades del fluido en el lecho estudiado. 

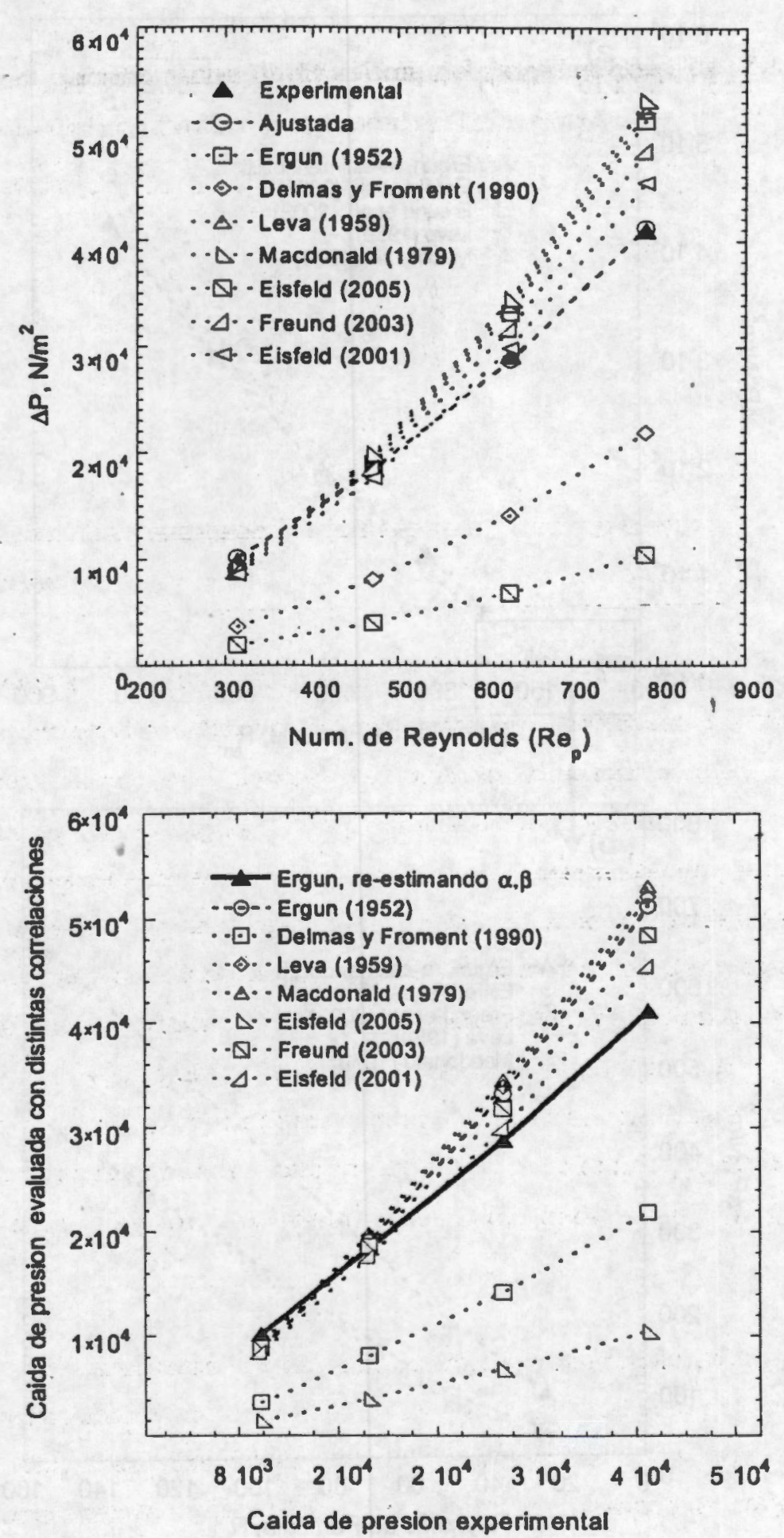

Figura 2.2. a) Comparación entre: la casda de presión experimental, la ajustada en este trabajo y la predicha con diferentes correlaciones propuestas en la literatura. b) Diagrama de paridad. 


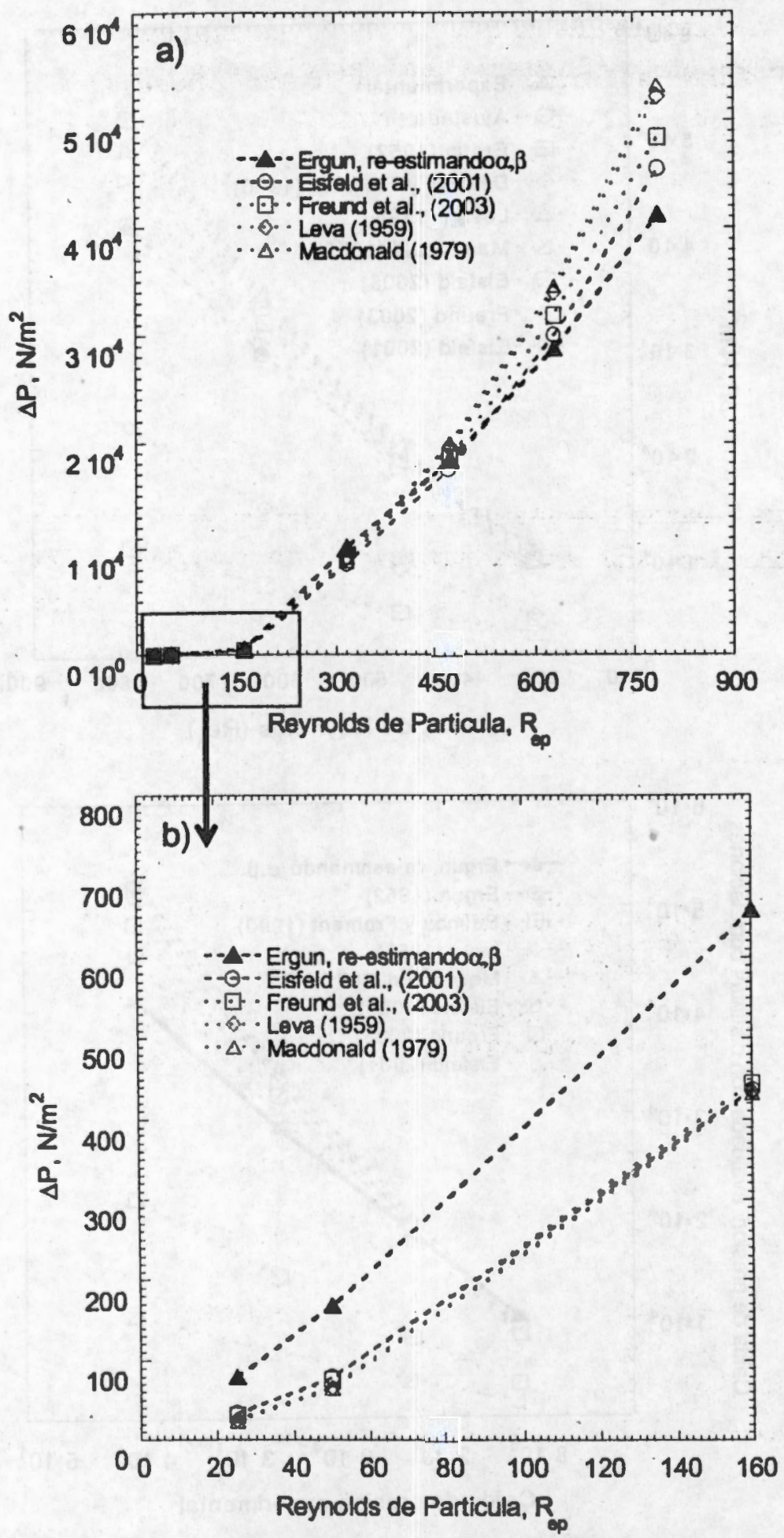

Figura 2.3. Comparación entre los datos de calda de presión con la correlación ajustada y otras correlaciones: a) $\operatorname{Re}_{\mathrm{p}}$ de 25 a $785 ;$ y b) $\operatorname{Re}_{\mathrm{p}}$ de 25 a 160 


\subsubsection{Perfiles de fracción vacia}

Para la descripción de los perfiles de fracción vacía, en los sistemas estudiados, se utilizan diferentes ecuaciones empiricas que han sido empleadas en la literatura para predecir los perfiles de fracción vacla en un lecho empacado con una baja relación de $d_{c} / d_{p}$. La Figura 2.4 muestra esta comparación. Para discutir estas predicciones, se recurrirá a la figura 2.1 que da una idea fisica de los perfiles tridimensionales de fracción vacía que se tiene en los sistemas estudiados. De las correlaciones propuestas en la literatura (ver Apéndice B), y de acuerdo con las Figuras 2.1 y 2.4, unicamente tres correlaciones $[44,59,63]$ muestran cualitativamente el comportamiento radial de la fracción vacia. Las cuales predicen dos regiones de alta fracción vacía: cerca de la pared del tubo y a un diámetro de partícula de ésta. Sin embargo, entre ellas hay diferencias que van del 5 al 204 , es decir, variaciones de amplitud y posición en los valores mínimos y máximos de la fracción vacia. De estas correlaciones una de las que mejor predice la variación radial de la fracción vacía es la de deKlerk (2003), la cual se ha construido a partir de una gran cantidad de resultados experimentales de sistemas con bajas y altas relaciones de $d_{e} / d_{p}$ [59]. En este estudio se uso esta ecuación para predecix los perfiles de fracción vacia que se muestra en la Figura 2.1. No obstante, en el Apéndice $D$ se presenta un estudio de sensibilidad de los perfiles de velocidad predichos en el lecho al utilizar diferentes correlaciones de fracción vacía propuestas en la literatura $[44,59,63]$, donde se muestra que una mala elección de la correlación de la fracción vacía se reflejaría en una inadecuada descripción de los perfiles de velocidad y los procesos de transporte de calor en el lecho de estudio (ver Apéndice G).

La literatura indica que la permeabilidad y el número de Darcy caracterizan a un lecho empacado, sin embargo, a diferencia de modelos de lechos empacados que utilizan una fracción vacía consțante, la variación de la permeabilidad y del numero de Darcy a lo largo del 
radio crean una incertidumbre en la elección del modelo que debe empelarse para predecir los campos de velocidad.

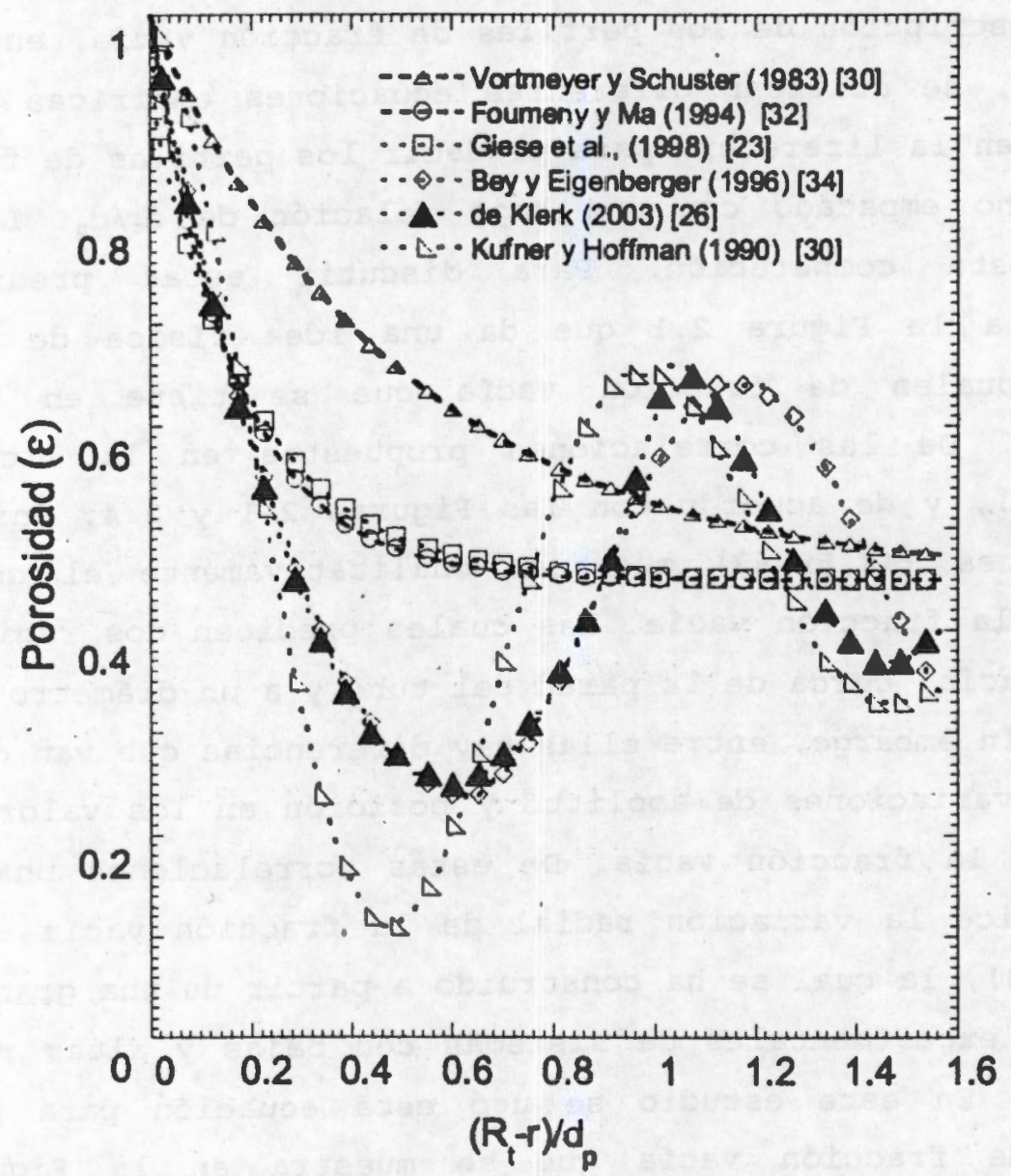

Figura 2.4. Predicción de los perfiles de fracción vacia usando diferentes correlaciones.

Por lo mencionado anteriormente, en este estudio es indispensable usar una expresión de permeabilidad que cualitativamente describa al sistema. En la literatura se han propuesto una gran cantidad de modelos empiricos para caracterizar la permeabilidad en lechos empacados, sin embargo, aún se tienen problemas para su predicción. La Figura 2.5a muestra una comparación entre las diferentes correlaciones propuestas en la literatura para predecir la permeabilidad en lechos empacados (ver Apéndice A). Dentro de estas Ecuaciones de permeabilidad se evaluo la Ecuación (2.15) con a igual a 1028, que es el valor estimado con Ios datos de caída de presión. En esta Figura se muestra el comportamiento 
$-7 w *^{\circ}$

de la permeabilidad, la cual varía a lo largo del radio, como resultado de la variación radial de la fracción vacia, y su magnitud depende de la ecuación utilizada. Todas las ecuaciones muestran un comportamiento similar, un máximo en la permeabilidad en secciones de alta fracción vacia. Sin embargo, la diferencia entre los resultados que arrojan las ecuaciones propuestas en la literatura y la permeabilidad re-estimada en este trabajo, que es la de Ergun re-estimando $\alpha$ (Ecuación 2.15; $\alpha$ igual a 1028), van entre 50 y 300\%, dependiendo de la correlación. Por lo que se decidió utilizar la Ecuación de permeabilidad modificada para calcular el número de Darcy y éste indicó un comportamiento noDarcyano, como se observa en la Figura 2.5b. En esta Figura se observa que el número de Darcy mostró un comportamiento similar a la permeabilidad, ya que existe entre éstos una relación proporcional con la fracción vacía.

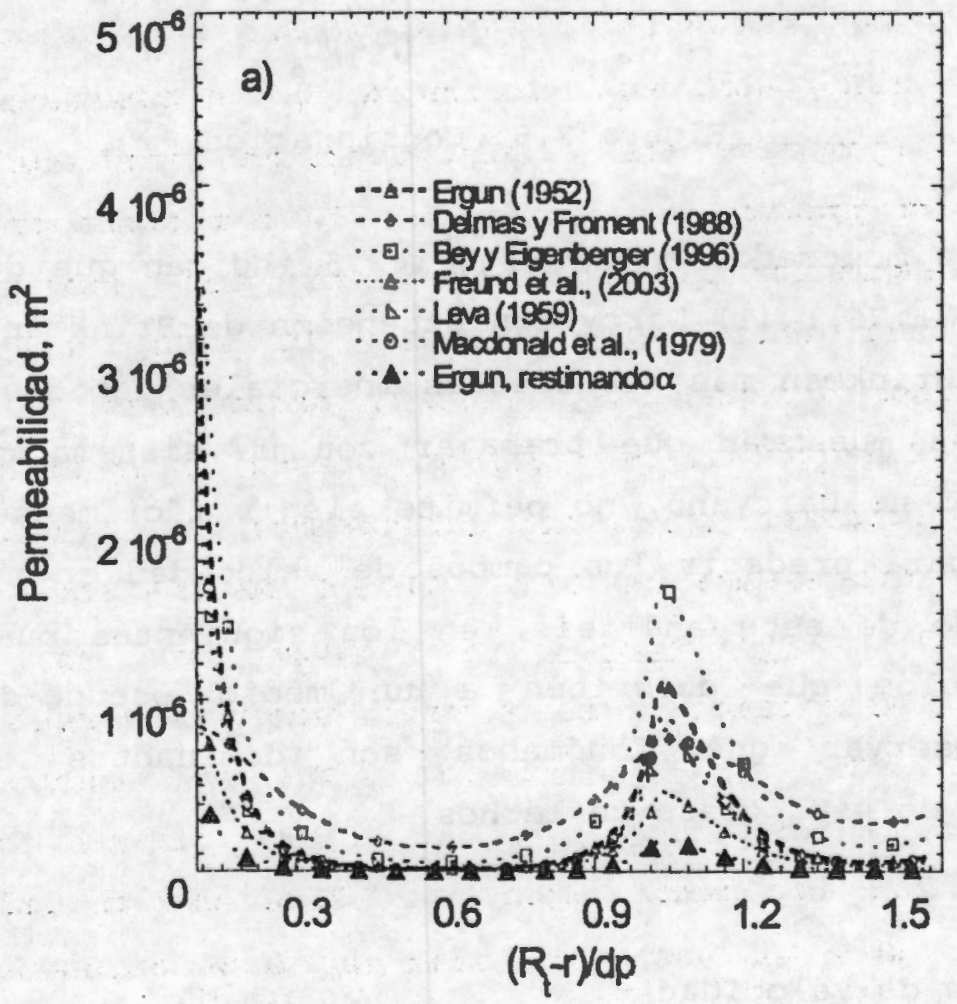

Figura 2.5. a) Las predicciones de la permeabilidad y b) el número de Darcy. 


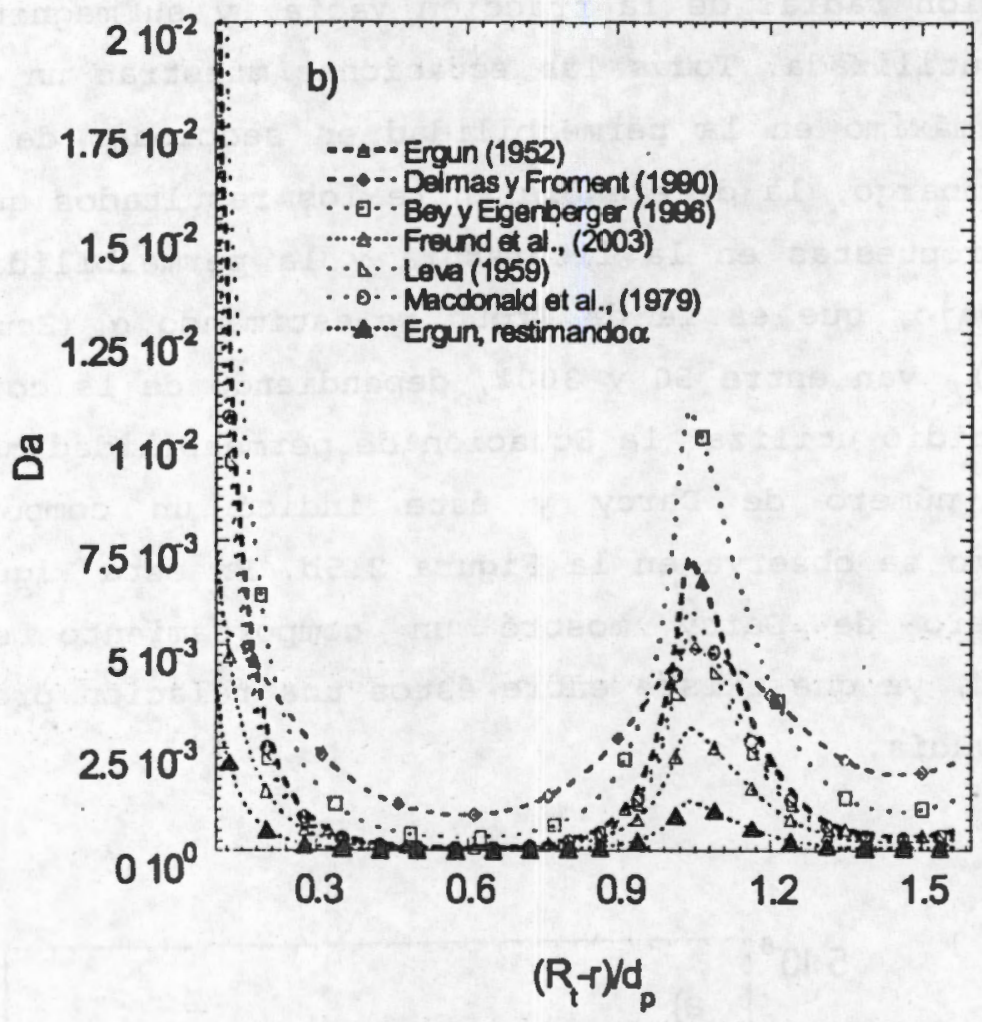

Figura 2.5. Continuación.

Los resultados mostrados en la Figura 2.5 indican que deben ser usados dos modelos [71,75]: (1) Darcy con extensión de Brinkman; (2) Darcy con extensión de Brinkman más 108 efectos inerciales debidos a Forchheimer. Los resultados muestran que trabajar con un sistema que presenta un comportamiento no Darcyano, no permite elegir fácilmente el modelo que se requiere para predecir los campos de velocidad en nuestro sistema. Como resultado de este análisis, en los siguientes puntos se evalúan diversos modelos que describen a un medio pseudo-continuo $y$ que permitirá observar que fenómenos son dominantes en el estudio hidrodinámico de esta clase de lechos.

\subsubsection{Perfiles de velocidad}

Desde un punto de vista hidrodinámico, los fenómenos que se han discutido hasta esta sección incluyen dos procesos los cuales se 
atribuyen a la interacción del fluido con las superficies s6lidas en un lecho empacado con una baja relación de $d_{t} / d_{p}$. Por un lado, un incremento en el flujo local debido a una alta fracción vacia $y$ por otro la reducción de la velocidad local a cero en las superficies sólidas. En principio, se puede decir que a flujos de alimentación bajos los efectos viscosos dominan por lo que estos son muy importantes. Mientras que a flujos de alimentación altos, los efectos viscosos suceden en una pequeña capa limite alrededor del sólido, por lo cual los efectos inerciales, particularmente en regiones con altas fracciones vacias, son más importantes. Tratando de estudiar estos fenómenos en la siguiente sección se presenta la predicción de los campos de velocidad en el lecho empacado a diferentes flujos de alimentación. Se destacan dos puntos; en el primero se hace un estudio de diferentes modelos reportados en la literatura para modelar un lecho empacado con una relación de $d_{c} / d_{p}<10$ (ver sección 2.1 ); en el segundo se hace un estudio de cada uno de los términos que intervienen en el modelo descrito en la sección 2.2 .

\subsubsection{Evaluación de los distintos modelos hidrodinámicos que se emplean en la literatura}

Debido a la gran demanda de recursos computacionales, los "métodos de ingeniería aproximados" se han empleado exitosamente a la fecha, para predecir los campos de velocidad en leohos empacados con una baja relación de $d_{t} / d_{p}$. Sin embargo, no existe una opinión uniforme acerca de cual de los modelos usados en la literatura es el más conveniente. A continuación se presentan los modelos que a saber se han empleado en la literatura para predecir los campos de velocidad en lechos empacados con una relación de $d_{c} / d_{p}$ menor a 10. Se mencionan sus consideraciones particulares y caracteristicas principales. Para facilitar la discusión de los modelos, éstos se codifican como 1-A hasta 1-E, $Y$ en adelante serán referidos con estas claves. 
El modelo 1-A utiliza las constantes de Ergun para considerar las interacciones entre el sólido-gas, se plantea y resuelve en términos de la velocidad superficial y se utiliza la viscosidad efectiva en el término viscoso de Navier-stokes para considerar las resistencias viscosas cerca de la pared del lecho. Este modelo esta dado por:

$$
\begin{aligned}
& \rho_{q}\left(\frac{\partial v}{\partial t}+v \cdot \nabla v\right)=-\nabla p+\tilde{\mu} \nabla^{2} v+R_{g-0} \\
& \frac{\tilde{\mu}}{\mu_{t}}=2 \exp \left(0.002 R_{e p}\right)
\end{aligned}
$$

El modelo $1-B$ a diferencia del modelo $1-A$ se plantea $y$ resuelve en términos de la velocidad intersticial. Este modelo esta dado por:

$\rho_{\varepsilon}\left(\frac{\partial \varepsilon u}{\partial t}+\varepsilon u \cdot \nabla \varepsilon u\right)=-\nabla \varepsilon p+\tilde{\mu}\left(\nabla^{2} \varepsilon u\right)+\mathbf{R}_{q-\bullet}$

El modelo $1-C$ a diferencia del modelo $1-B$ omite el uso de la viscosidad efectiva en el termino viscoso de Navier-stokes utilizando la viscosidad dinámica del fluido. Este modelo esta dado por:

$\rho_{\varepsilon}\left(\frac{\partial \varepsilon u}{\partial t}+\varepsilon u \cdot \nabla \varepsilon u\right)=-\nabla \varepsilon p+\mu_{\varepsilon}\left(\nabla^{2} \varepsilon u\right)+R_{g-1}$

El modelo $1-D$ a diferencia del modelo anterior utiliza los valores reestimados de $\alpha$ y $\beta$ dentro de los términos viscoso e inercial de la ecuación de Ergun. Este modelo esta dado por:

$\frac{\partial \rho_{\varepsilon} \varepsilon u}{\partial t}+\nabla \cdot\left(\varepsilon \rho_{\varepsilon} u u\right)=-\nabla(\varepsilon p)+\nabla \cdot(\varepsilon \tau)+\mathbf{R}_{g \cdot \bullet}$

El modelo 1-E a diferenciad del modelo $1-D$, muestra el efecto de la fracción vacía en el término viscoso de Navier-stokes: 
$\frac{\partial}{\partial t}\left(\rho_{\varepsilon} \varepsilon u\right)+\rho_{\varepsilon} \varepsilon u \cdot(\nabla \varepsilon u)=-\nabla \varepsilon p+\mu_{f} \nabla^{2} \varepsilon u+\rho_{q} g+R_{g-0}$

En la Bigura 2.6 se presentan los perfiles radiales de velocidad axial, para un rango de $R e_{p}$ de 25 a 785, predichos con los modelos 1-A (Figura. 2.6a) Y 1-B (Figura 2.6b). El modelo 1-A fue utilizado por Delmas $y$ Froment [85], Daszkowski y Eigenberger [61], Papageorgiou y Froment [30], Giese et al. [56], entre otros. El modelo 1-B Eue considerado como lo hace Foumeny y Ma [62], Bey y Eigenberger [63], Eisfeld et al. [60]. La viscosidad efectiva es una función del número de Reynolds y de la viscosidad dinámica del fluido. Para predecir su valor, se tomo la expresión reportada por Winterberg y Tsotsas (Ecuación (2.31)) [54]. Los resultados que se obtienen al comparar las predicciones de los perfiles de velocidad, entre los modelos $1-A$ y $1-B$, se discuten a continuación.

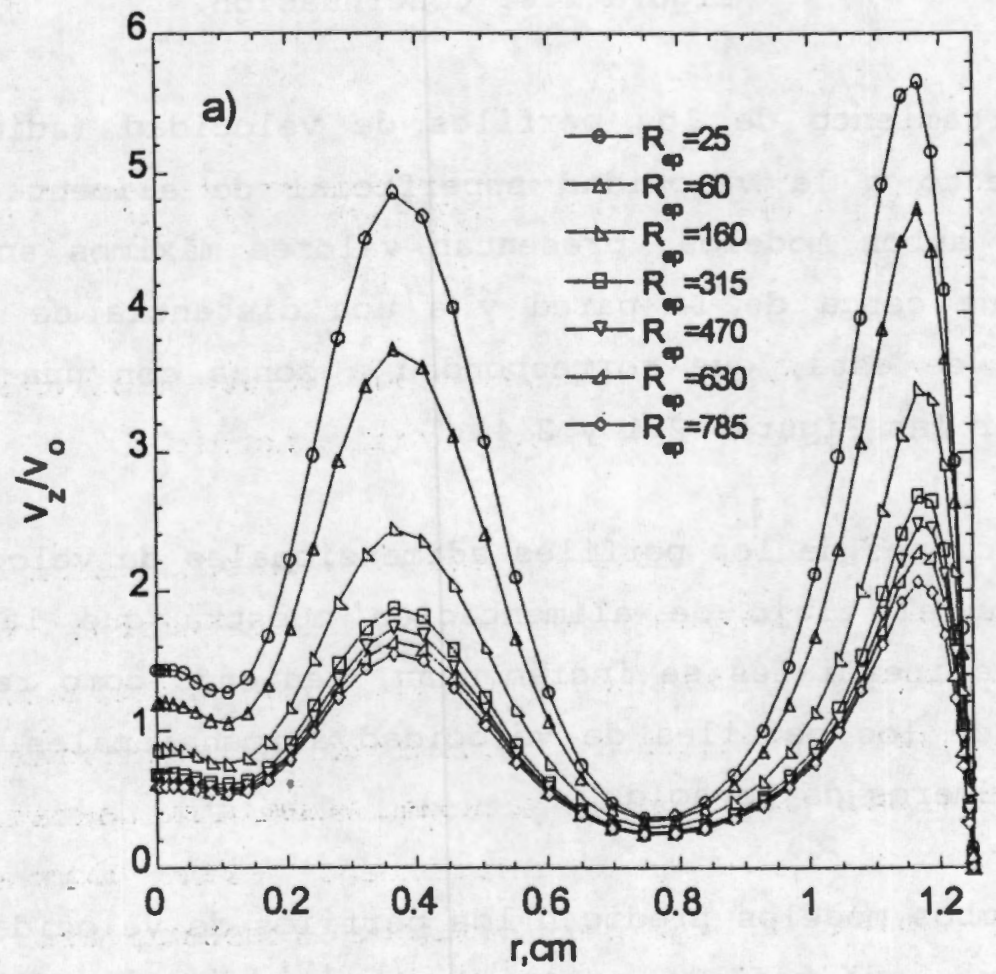

Figura 2.6. Predicción de los perfiles radiales de velocidad axial, para diferentes $\mathrm{Re}_{\mathrm{p}}$ : a) modelo 1-A y b) modelo 1-B. 


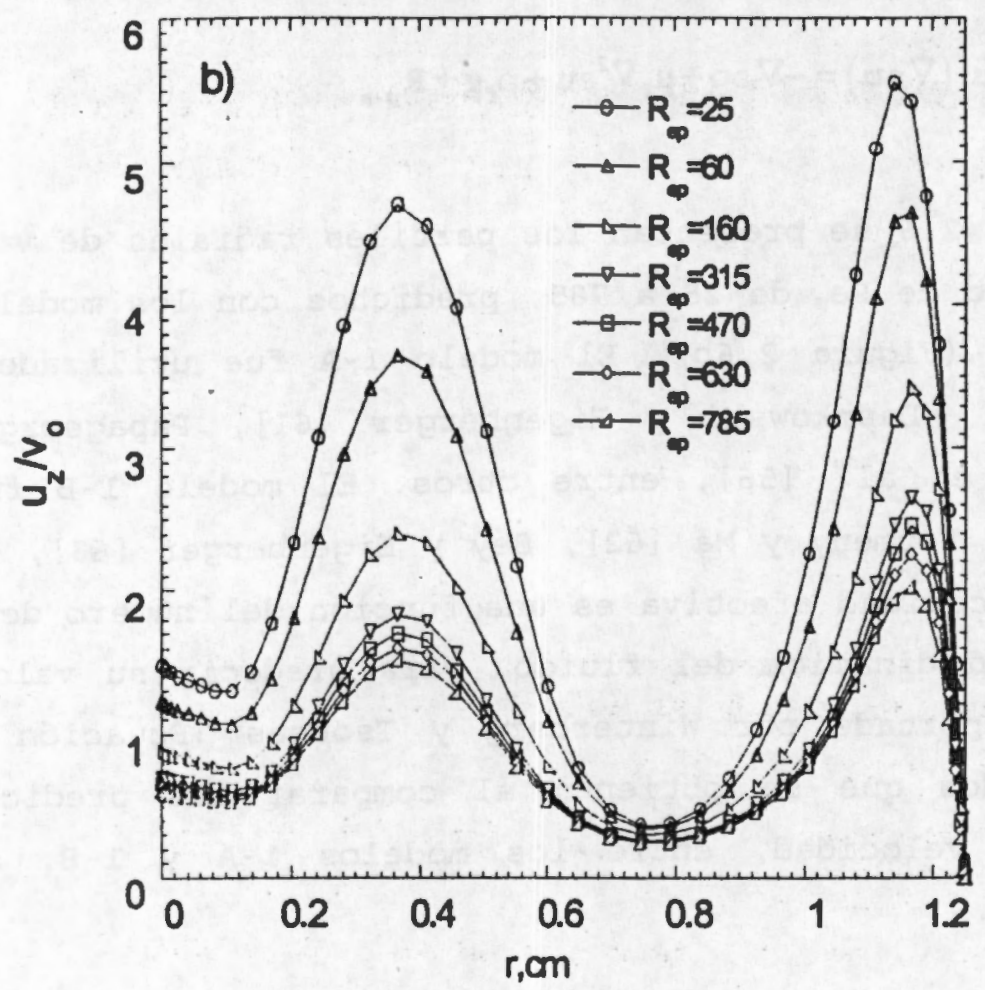

Figura 2.6. Continuación.

1. El comportamiento de los perfiles de velocidad (adimensionalizados con respecto a la velocidad superficial de alimentación al lecho, $\mathrm{V}_{0}$ ), para ambos modelos, presentan valores máximos en la velocidad, en la zona cerca de la pared y a una distancia de un diámetro de particula de esta, que corresponden a zonas con una mayor fracción vacia (ver las Figuras 2.1 y 2.4).

2. Las variaciones de los perfiles adimensionales de velocidad cuando se incrementa el flujo de alimentación muestra que las resistencias viscosas e inerciales se incrementan, teniendo como resultado que la magnitud de los perfiles de velocidad adimensionales sean menores a mayores números de Reynolds.

3. Si bien ambos modelos predicen los perfiles de velocidad en una forma cualitativa, que refleja a este lecho empacado (ver Figura 2.1), se encontró que hay diferencias de alrededor del 1 a 5 t entre ambos, 
dependiendo de la posición radial y del flujo de alimentación. En este mismo sentido, se debe tomar en consideración la incertidumbre que existe en la evaluación de la viscosidad efectiva.

4. Al considerar una velocidad superficial y no intersticial, se pensaría que se tiene un problema mal planteado, ya que se esta trabajando con un lecho empacado. Sin embargo, las predicciones dan aproximadamente los mismos resultados. Lo anterior señala que la derivada de la fracción vacía con respecto al radio (ecuaciones (2.19)-(2.25)), cuando se considera a la velocidad intersticial, tiene una magnitud despreciable, comparadas con los términos viscoso e inercial considerados con la ecuación de Ergun.

Lo anterior lleva a preguntarnos: ¿los terminos de Ergun (que incorporan a las interacciones solido-gas), describen a las resistencias viscosas e inerciales en lechos empacados con un baja relación de $d_{t} / d_{p}$, de tal forma que se puede omitir el uso de una viscosidad efectiva?. Esta pregunta nos lleva a evaluar al modelo 1-C, el cual utiliza a la velocidad intersticial y omite el uso de la viscosidad efectiva. En la Figura 2.7 se presentan los perfiles radiales del componente axial de la velocidad, predichos bajo las condiciones mencionadas anteriormente. Una comparación entre las Figuras 2.7, 2.6a y 2.6b, muestran que los perfiles de velocidad adimensionales, cuando se omite la viscosidad efectiva, son mayores, que cuando se le considera; las diferencias son alrededor del 15 al $60 \%$ a flujos bajos, $y$ de 1 a $15 \%$ para flujos altos. Estos resultados indican que los términos originales de la expresión de Ergun fueron estimados considerando un sistema pseudo-homogéneo con una fraccion vacía no mayor a 0.5 , de esta manera, las resistencias viscosa e inercial fueron caracterizadas para un lecho empacado con diferentes empaques y flujos de alimentación que los utilizados en este estudio. El lecho usado en este trabajo presenta secciones a lo largo del radio con una fracción vacía mayor a 0.5 , lo que hace que las superficies sólidas presenten resistencias tanto viscosas como inerciales diferentes a las del sistema utilizado por Ergun. Es tal vez por esto 
que algunos investigadores han usado, para bajas relaciones de $d_{t} / d_{p}$, el término de viscosidad efectiva y la relación de Ergun. Sin embargo, y como se muestra en la sección siguiente, cuando se trabaja con un sistema con una relación de $d_{c} / d_{p}$ igual a 3.15 , los diferentes enfoques, al usar una viscosidad efectiva o re-estimando los parámetros $\alpha$ y $\beta$ de la ecuación de Ergun, no predicen los mismo perfiles de velocidad en zonas alejadas de la pared.

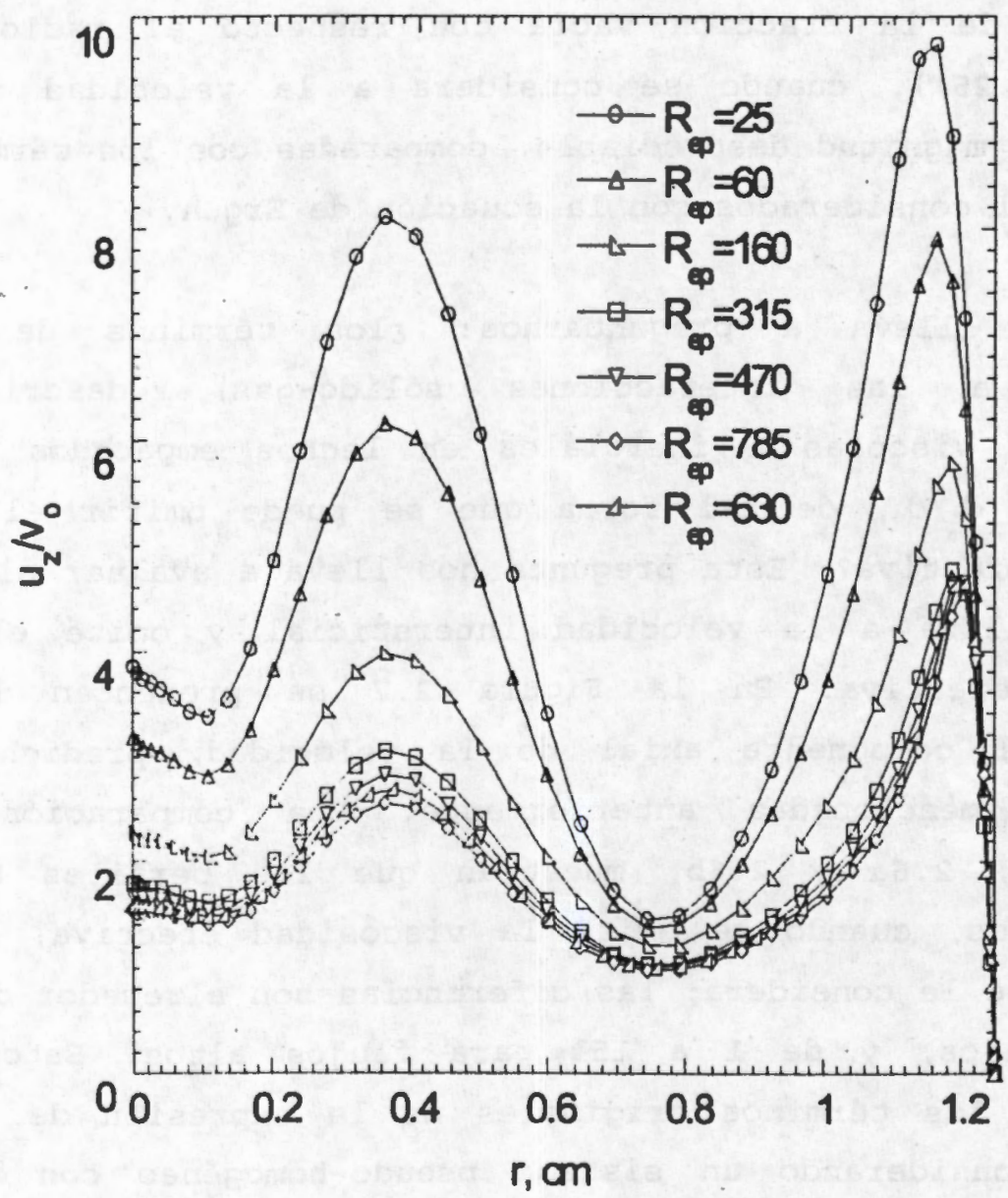

Figura 2.7. Predicción de los campos de velocidad axial a lo largo del radio, empleando diferentes $R_{p}$ y el modelo $1-C$.

En la Figura 2.8 se presentan las predicciones de los modelos $1-D$ y $1-E$ que consideran el empleo de una velocidad intersticial, se omite la viscosidad efectiva $y$, los valores $\alpha$ y $\beta$ propuestos por Ergun se re- 
ajustan para considerar las interacciones entre el solido y fluido del lecho estudiado, 1028 y 1.15 en vez de 150 y 1.75, respectivamente. La diferencia entre el modelo $1-D Y$ Y $1-E$, son algunos términos que involucran la fracción vacia. Sin embargo, las predicciones entre estos modelos son despreciables, de alrededor de 1-7 a flujos bajos y de 1 37 a flujos altos dependiendo de la posición radial. Una comparación entre las predicciones por estos modelos y el $1-C$, muestra que se tienen diferencias mayores al 100\%. Lo anterior señala la necesidad de re-estimar los efectos de las resistencias inerciales $y$ viscosas debido a la presencia del solido, ya que son dos términos dominantes en la predicción de los perfiles de velocidad en lechos empacados con bajas relaciones de $d_{t} / d_{p}$. Una observación es que al predecir los perfiles de velocidad con el modelo 1-D y 1-E, en comparación con los modelos 1-A a 1-C, para un Rep mayor o igual a 315, los perfiles de velocidad adimensionales no cambian, por lo que las resistencias viscosas e inerciales inciden en la misma magnitud en la predicción de los perfiles de velocidad a flujos altos.

Es importante aclarar nuevamente que si bien los perfiles de velocidad adimensionales son mayores a flujos bajos de alimentación, a lo largo del radio, estos únicamente reflejan el efecto de las resistencias viscosas e inerciales, ya que cuando estos perfiles se presentan en términos de la velocidad dimensional, un flujo alto de alimentación corresponde a una mayor velocidad intersticial a lo largo del radio [47]. 

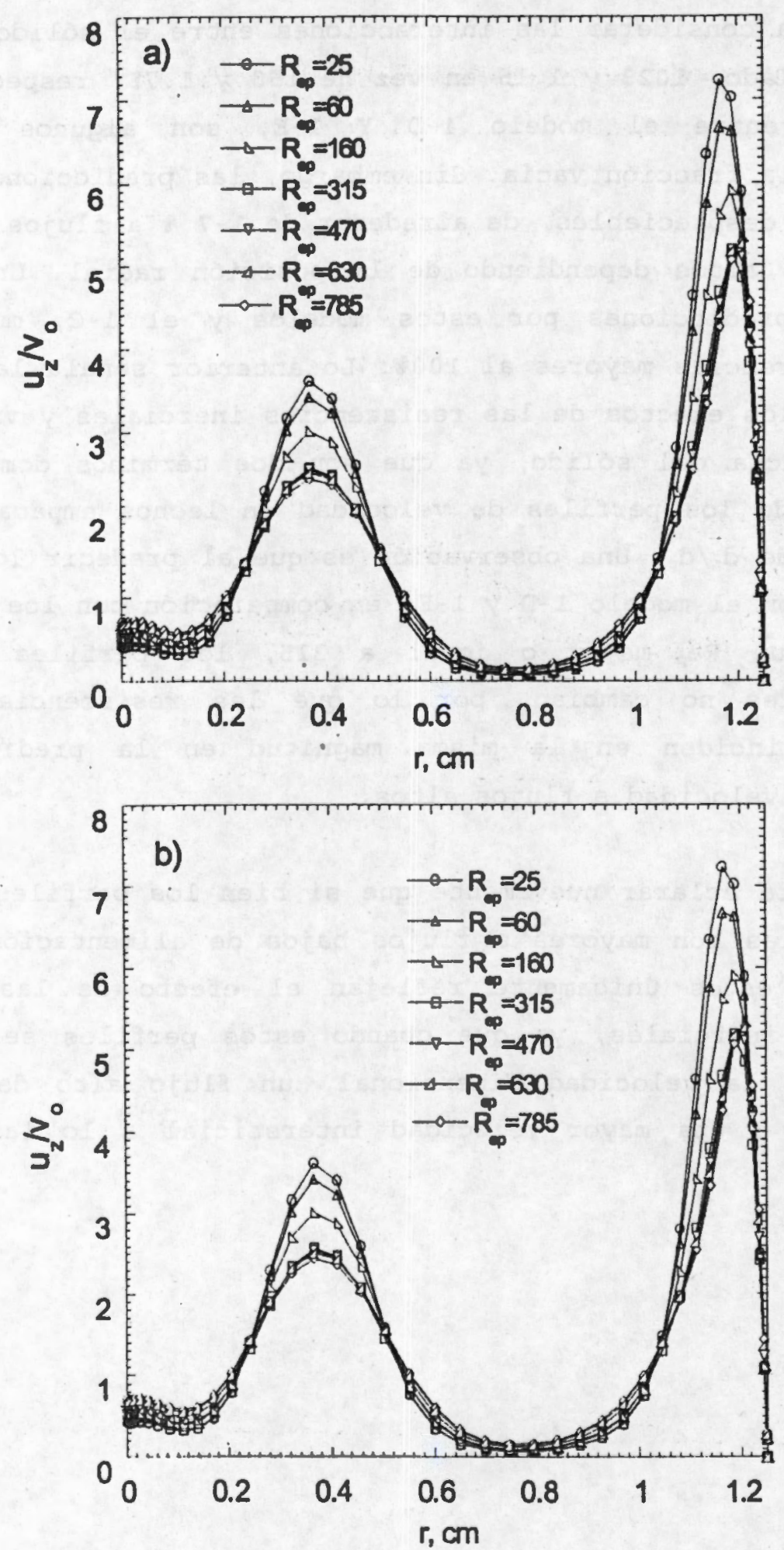

Figura 2.8. Predicción de los campos de velocidad axial a lo largo del radio, para diferentes $R e_{p}$ : a) modelo $I-D$ y b) modelo $1-E$. 
En la Figura 2.9 se presenta una comparación de los modelos 1A-1E, para dos $\operatorname{Re}_{\mathrm{p}}$ (25 y 785), cuyas predicciones se mostraron en las Figuras 2.62.8. Al observar estos resultados, la pregunta es: za pesar de las diferencias entre los modelos, existe algun têrmino que tenga que modificarse para que estos modelos puedan predecir el mismo perfil de velocidad?. Como no se tienen resultados experimentales se supone, con base en la literatura [47], que las predicciones del modelo 1-E son correctas, ya que la magnitud de los efectos inerciales y viscosos se considera a partir de experimentos de caida de presión y se maneja en términos de la velocidad intersticial [60]. Para dar respuesta a esta pregunta se propone la siguiente estrategia:

a) Para que el modelo 1 -A prediga los mismos resultados del modelo $1 E$, la viscosidad efectiva debe ser re-estimada.

b) Para que el modelo 1-B, que considera la velocidad intersticial y los parámetros de Ergun, prediga los resultados del modelo 1-E, también debe reajustarse la viscosidad efectiva, lá cual presentará pequeñas diferencias con respecto a la del modelo 1-A.

c) Para que el modelo 1-C prediga los resultados del modelo 1-E, los parámetros de Ergun deben re-estimarse, y obviamente se teridrá el modelo $1-E$.

d) Finalmente para que el modelo 1D, de los mismos resultados que el modelo 1-E los parámetros ya re-estimados deben tener un pequeño ajuste para reducir las pequeñas diferencias que se tienen.

El objetivo de hacer esta comparación es mostrar que los diferentes modelos que se emplean en la literatura, que pueden no estar adecuadamente planteados, puedan ser utilizados en la predicción de los perfiles de velocidad en un lecho empacado, unicamente si se hace una consideración importante en el modelo empleado. En la Figura 2.10 se muestra una comparación entre los modelos 1-A a $1-E$ explicando las sugerencias anteriores $y$ utilizando un $\mathrm{Re}_{\mathrm{p}}$ igual a 785 . 

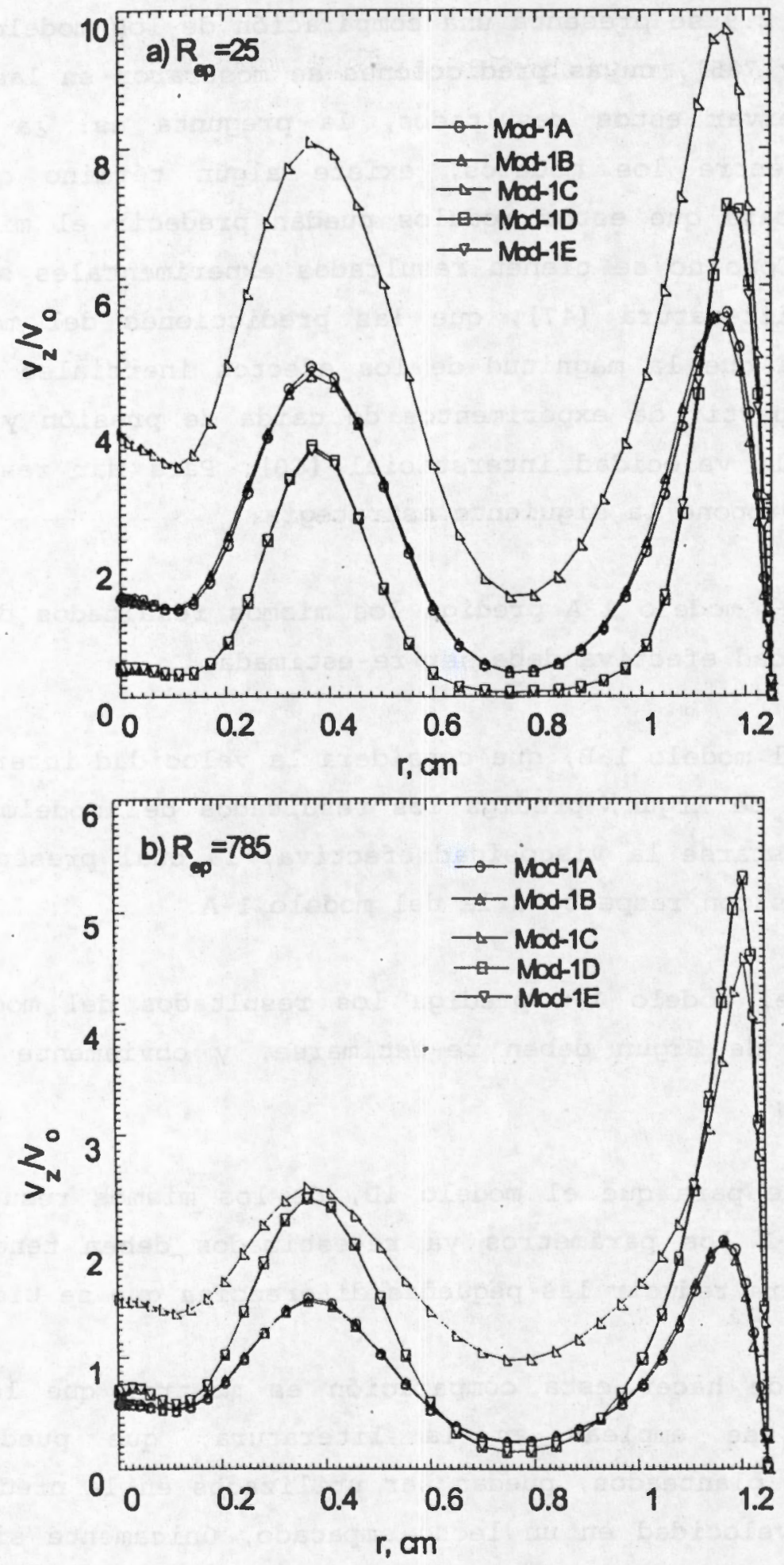

Figura 2.9. Predicción de los campos de velocidad axial a lo largo del radio con los modelos $1 \mathrm{~A}-1 \mathrm{E}$ : a) $R e_{p}=25$ Y b) $R e_{p}=785$ 

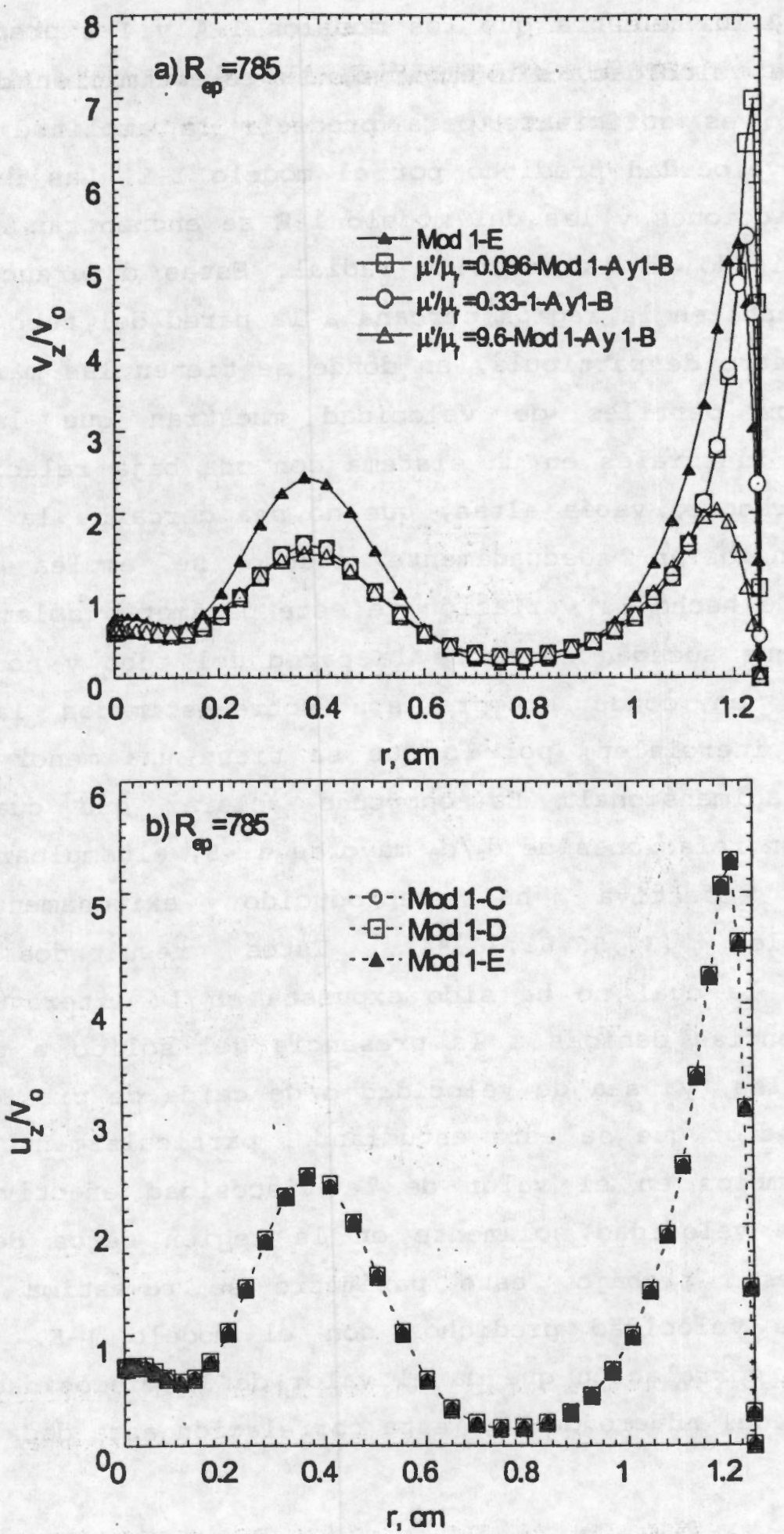

Figura 2.10. Predicción de los campos de velocidad axial a lo largo del radio estimando: a) la viscosidad efectiva $y$ b) los parámetros $\alpha$ y $\beta$. 
La Figura 2.10 a muestra que los modelos 1-A y 1-B predicen los mismos perfiles de velocidad, sin embargo, la re-estimación de la viscosidad efectiva no es suficiente para predecir la amplitud $Y$ magnitud del perfil de velocidad predicho por el modelo $1-E$. Las diferencias entre estas predicciones y las del modelo $1-E$ se encuentran alrededor del 2807 dependiendo de la posición radial. Estas diferencias se observan principalmente en la región cercana a la pared del tubo y a la posición de un diámetro de partícula, en donde se tienen las máximas fracciones vacias. Los perfiles de velocidad muestran que las resistencias viscosas e inerciales en un sistema con una baja relación de $d_{k} / d_{p}$, en zonas de fracción vacía altas, que no sea cerca de la pared del tubo, no se consideran adecuadamente cuando se emplea una viscosidad efectiva. De hecho la variación de este parámetro solamente afecta los fenómenos que suceden cerca de la pared del tubo $y$ no en el interior del lecho, en donde siempre son sobre-estimados las resistencias viscosas e inerciales, por lo que se tiene una menor magnitud en la velocidad adimensional. Es oportuno aclarar que cuando se tienen sistemas con relaciones de $d_{\varepsilon} / d_{p}$ mayores a -5 , el emplear el concepto de viscosidad efectiva ha reproducido exitosamente resultados experimentales $[37,56,61,76,84]$. Estos resultados muestran la relevancia, la cual no ha sido expuesta en la literatura, de estimar las resistencias debidas a la presencia del solido a partir de datos experimentales, ya sea de velocidad o de calda de presión $[27,76]$, del lecho o reactor que se esta estudiando, particularmente con $d_{\mathrm{r}} / d_{\mathrm{p}}<5$. Si bien cambios en el valor de la viscosidad efectiva modifica los perfiles de velocidad solamente en la región cerca de la pared del tubo, en este trabajo, este parámetro se re-estima utilizando los perfiles de velocidad predichos con el modelo $1-\mathrm{E}$, con lo que se propuso una correlación que da el valor de la viscosidad efectiva como una función del número de Rep, esta correlación esta dada por:

$$
\frac{\tilde{\mu}}{\mu_{q}}=0.007 \exp \left(0.0025 R_{e p}\right)
$$


La Figura 2.11 muestra una comparación de las predicciones de esta correlación con respecto a la reportada en la literatura [56]. Las diferencias entre estas correlaciones son importantes y muestran la necesidad de re-estimar este parámetro para cada lecho en particular. Sin embrago, los resultados expuestos arriba ponen en duda el uso de este parámetro en la predicción de perfiles de velocidad en lechos de esta clase, por lo que estudios, contando con mediciones experimentales de la velocidad, tienen que hacer realizados para abordar las observaciones realizadas.

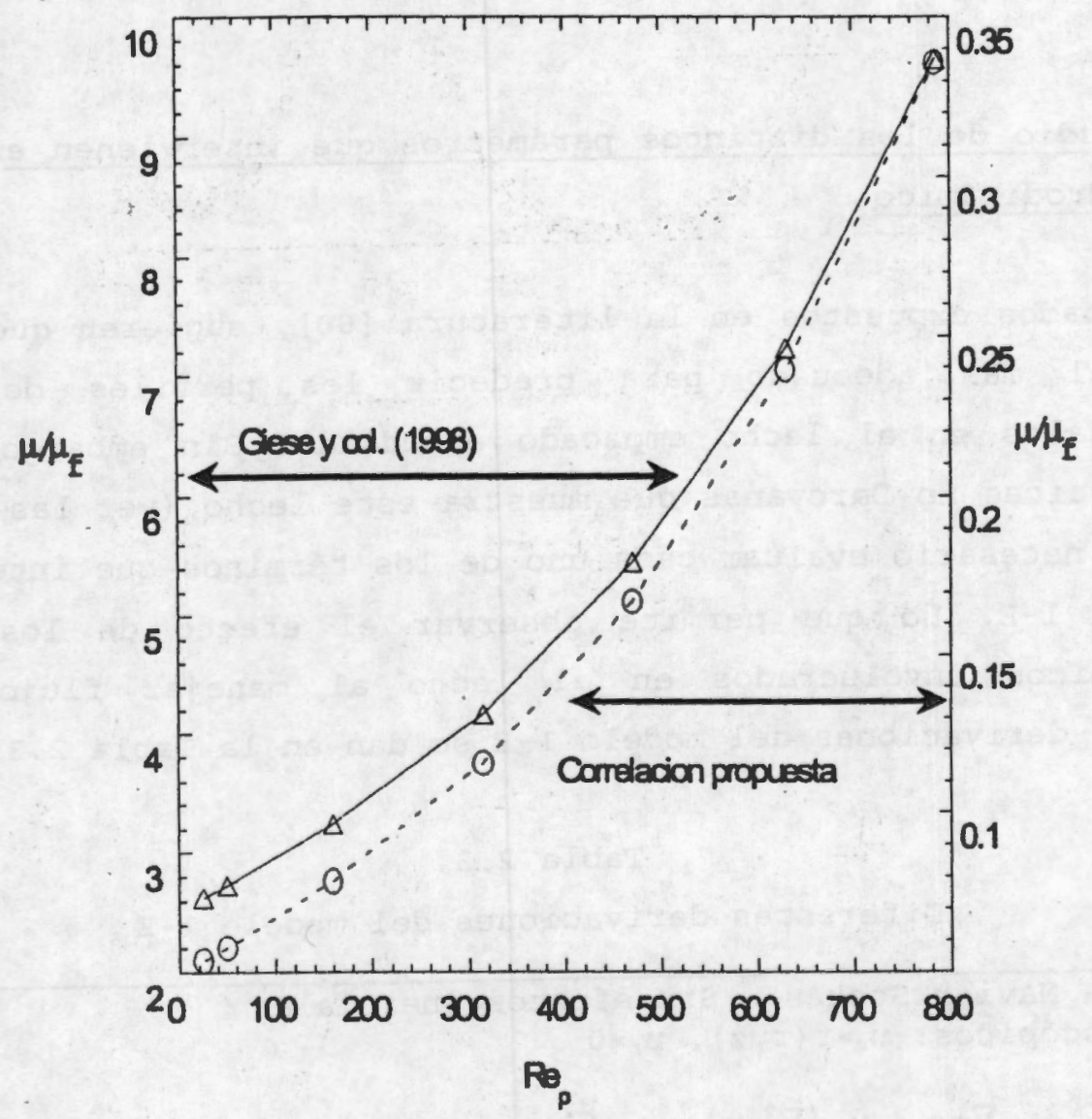

Figura 2.11. Comportamiento de la viscosidad efectiva.

En la Figura $2.10 \mathrm{~b}$ se muestra que los modelos $1-\mathrm{C}$ y $1-\mathrm{D}$ predicen adecuadamente los resultados predichos por el modelo $1-\mathrm{E}$, esto es porque ambos modelos tienen como parámetros de ajuste $\alpha$ Y $\beta$ los cuales 
son claves para considerar los efectos viscosos e inerciales en un lecho empacado con una fracción vacia alta.

En esta sección se puede notar que la estimación de los parámetros que consideran los efectos inerciales $y$ viscosos debidos a Darcy $y$ Forchheimer deben ser estimados en cada sistema de estudio, por ejemplo de datos de caída de presión. Este procedimiento resulta ser más confiable que utilizar la viscosidad efectiva, la cual, excluyendo la zona cercana a la pared del tubo, no podría predecir los perfiles de velocidad, particularmente en sistemas con relaciones de $d_{\varepsilon} / d_{p}<5$.

\subsubsection{Estudio de 108 distintos parámetros que intervienen en el modelo hidrodinámico}

Los resultados expuestos en la literatura [60], sugieren que el modelo 1-E es el más adecuado para predecir los perfiles de velocidad intersticiales en el lecho empacado estudiado. Sin embargo, para las caracteristicas no Darcyanas que muestra este lecho (ver las Figura 2.1 Y 2.5) es necesario evaluar cada uno de los términos que intervienen en el modelo 1-E. Lo que permite observar el efecto de los fenómenos hidrodinámicos involucrados en el lecho al manejar flujos bajos y altos. Las derivaciones del modelo $1-\mathrm{E}$ se dan en la Tabla 2.3.

Tabla 2.3.

Diferentes derivaciones del modelo $1-\mathrm{E}$

2-A Darcy- Navier Stokes - Sin efectos inerciales macroscópicos: $u_{z}=f(x, z), u_{x}=0$

$\rho_{t} \frac{\partial \varepsilon u}{\partial t}=-\nabla \varepsilon p+\mu_{t}\left(\nabla^{2} \varepsilon u\right)-\frac{\mu_{\varepsilon}}{k(\varepsilon) d_{p}^{2}} \varepsilon u$

2-B Darcy- Navier Stokes: $u_{z}=f(r, z), u_{r}=0$

$p_{\varepsilon}\left(\frac{\partial \varepsilon u}{\partial t}+\varepsilon u \cdot \nabla \varepsilon u\right)=-\nabla \varepsilon p+\mu_{\varepsilon}\left(\nabla^{2} \varepsilon u\right)-\frac{\mu_{p}}{k(\varepsilon) d_{p}{ }^{2}} \varepsilon u$ 
(Continuation Tabla 3)

2-C Darcy-Forcheimer Navier Stokes: $u_{x}=f(r, z), u_{r}=0$

$$
\begin{aligned}
& \rho_{\varepsilon}\left(\frac{\partial \varepsilon u}{\partial t}+\varepsilon u \cdot \nabla \varepsilon u\right)=-\nabla \varepsilon p+\mu_{z}\left(\nabla^{2} \varepsilon u\right)-\frac{\mu_{l}}{k(\varepsilon) d_{p}^{2}} \varepsilon u \\
& -\frac{\rho_{\varepsilon}}{k_{2}(\varepsilon) d_{p}} \varepsilon u^{2}
\end{aligned}
$$

2-D Forcheimer- Navier Stokes: $u_{z}=f(r, z), u_{r}=0$

$$
\rho_{\varepsilon}\left(\frac{\partial \varepsilon u}{\partial t}+\varepsilon u \cdot \nabla \varepsilon u\right)=-\nabla \varepsilon p+\mu_{\varepsilon}\left(\nabla^{2} \varepsilon u\right)-\frac{\rho_{\varepsilon}}{k_{2}(\varepsilon) d_{p}} \varepsilon u^{2}
$$

2-E Darcy-Forcheimer Navier Stokes - sin

efectos viscosos macroscópicos axiales : $v_{z}=f(r, z), v_{r}=0$

$$
\begin{aligned}
\rho_{\varepsilon}\left(\frac{\partial \varepsilon u}{\partial t}+\varepsilon u \cdot \nabla \varepsilon u\right)=-\nabla \varepsilon p+\mu_{\varepsilon}\left(\nabla^{2} \varepsilon u\right) & -\frac{\mu_{\varepsilon}}{k(\varepsilon) d_{p}{ }^{2}} \varepsilon u \\
& -\frac{\rho_{\varepsilon}}{k_{2}(\varepsilon) d_{p}} \varepsilon u^{2}
\end{aligned}
$$

2-F Darcy-Forcheimer Navier Stokes en dos dimensiones: $u_{z}=f(r, z), u_{r}=f(x, z)$

$$
\begin{aligned}
\rho_{\varepsilon}\left(\frac{\partial \varepsilon U}{\partial t}+\varepsilon u \cdot \nabla \varepsilon u\right)=-\nabla \varepsilon p+\mu_{t}\left(\nabla^{2} \varepsilon u\right) & -\frac{\mu_{\varepsilon}}{k(\varepsilon) d_{p}^{2}} u \\
& -\frac{\rho_{\varepsilon}}{k_{2}(\varepsilon) d_{p}} \varepsilon u^{2}
\end{aligned}
$$

2-G Darcy-Forchheimer

$$
\frac{\partial \varepsilon u}{\partial t}=-\nabla \varepsilon p-\frac{\mu_{\varepsilon}}{k(\varepsilon) d_{p}^{2}} \varepsilon u-\frac{p_{\varepsilon}}{k_{2}(\varepsilon) d_{p}} \varepsilon u^{2}
$$

E1 modelo 2-A considera los términos viscosos (de Darcy y el considerado con la ecuación de Navier-stokes) y omite los términos inerciales (de Forchheimer $y$ de la ecuación de Navier-stokes). El modelo 2-B, a diferencia del modelo 2-A, incluye el término inercial de la ecuación de Navier-Stokes. Para observar el efecto de las resistencias inerciales definidas con el término de Forchheimer, este término fue incluido al modelo 2-B proponiendo el modelo 2-C. Con la idea de conocer la importancia de los efectos viscosos el término de Darcy se desprecia y únicamente se consideraron los términos inerciales de la ecuación de Navier-Stokes y el de Forchheimer, este modelo fue codificado como 2-D. El modelo 2-E, a diferencia del modelo 2-C, no 
considera el término viscoso axial contenido en la ecuación de NavierStokes. Para el lecho considerado, que presenta altas fracciones vacias, la necesidad de considerar la componente radial de la velocidad es inevitable, por lo que se decidio incorporarla dentro del modelo hidrodinámico, éste se codifica como 2-F. En la sección anterior se observo que los términos de Darcy y Forchheimer son dominantes en la predicción de los perfiles de velocidad. Con base en esto se decidió proponer un modelo que únicamente considera estos dos términos: el modelo 2-G.

En la Figura 2.12 se presenta la predicción de los perfiles de velocidad intersticial al emplear los modelos 2-A y 2-B, para el intervalo de $\mathrm{Re}_{\mathrm{p}}$ de 25 a 785. La comparación de estos modelos muestra que el término inercial de la ecuación de Navier-stokes, no tiene ninguna influencia en la predicción de los perfiles de velocidad a lo largo del radio. Ya que los perfiles de velocidad obtenidos por ambos modelos son idénticos. De tal forma que, este termino es despreciable comparado con el de Darcy. Asimismo, los perfiles de velocidad predichos con los modelos 2-A y 2-B son mayores en un 150\% a aquéllos predichos con el modelo 1-E (Figura 2.8b), esto indica que los efectos inerciales, considerados con el termino de Forchheimer en el modelo 1E, son dominantes a flujos bajos $y$ altos en el lecho estudiado. En la Figura 2.13 se muestra la comparación. entre los perfiles de velocidad axial adimensional predichos con los modelos 2-C y 2-D a dos diferentes números de Reynolds $\left(R e_{p}=25\right.$ y $\left.R e_{p}=750\right)$. Los perfiles de velocidad predichos con el modelo 2-D indican que las resistencias viscosas juegan un papel dominante en su predicción, ya que si estas se desprecian (no considerando el termino de Darcy), no se obtienen los perfiles de velocidad obtenidos con el modelo $2 \mathrm{C}$. A un número de Reynolds bajo (Figura 2.13a) las diferencias entre estos dos modelos son de un $100 \%$, mientras que a un número de Reynolds alto. (Figura $2.13 b)$ los perfiles de velocidad son iguales cerca de la pared del tubo $y$ diferentes en un $30 \%$ en la parte central. Estos resultados muestran que los efectos viscosos e inerciales que se consideran con la ecuación de Ergun, son indispensables a números de Reynolds bajos y altos. 

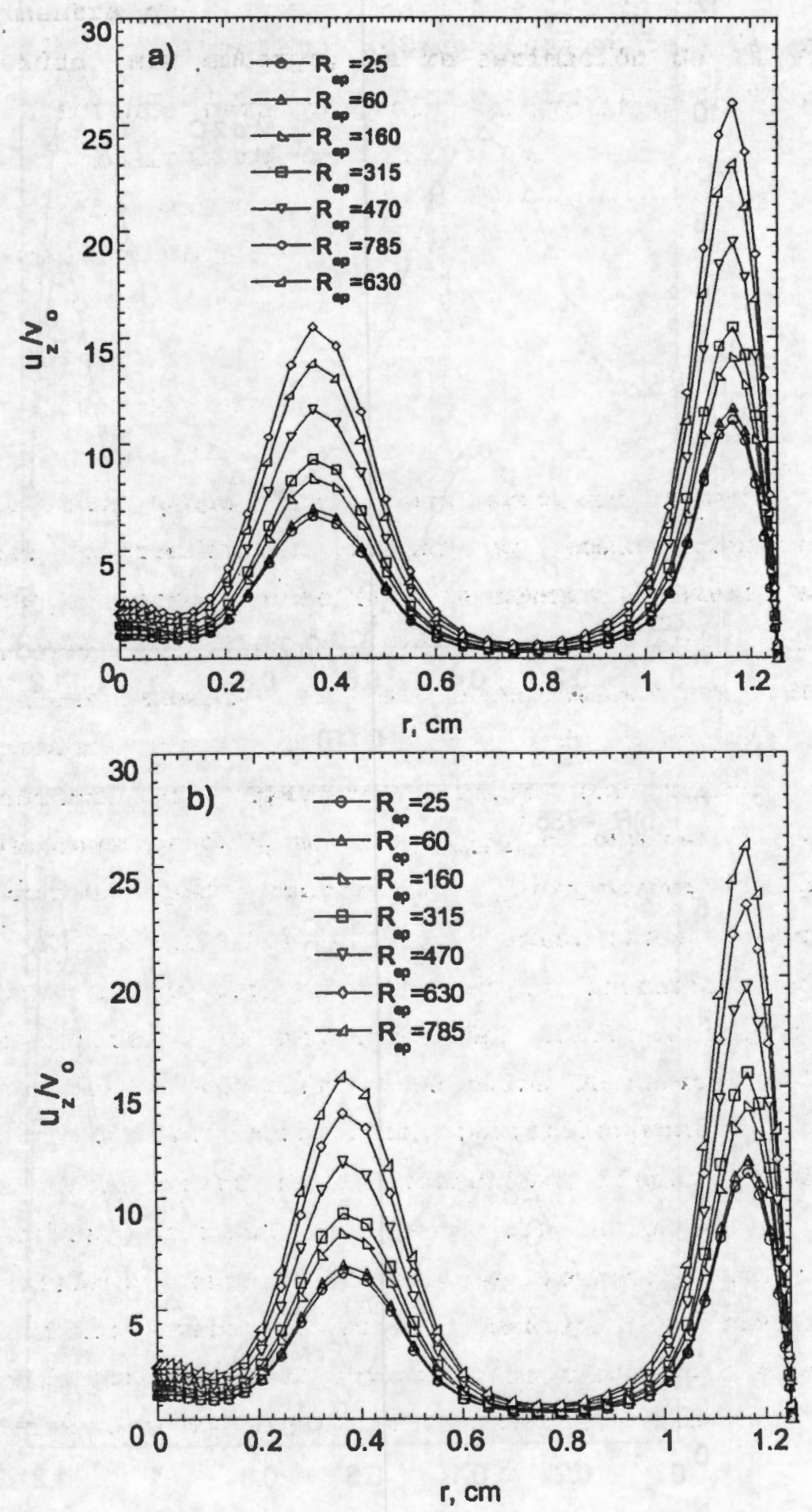

Figura 2.12. Predicción de los campos de velocidad axial a lo largo del radio, para diferentes $R e_{p}:$ a) modelo $2-A$ y b) modelo $2-B$. 

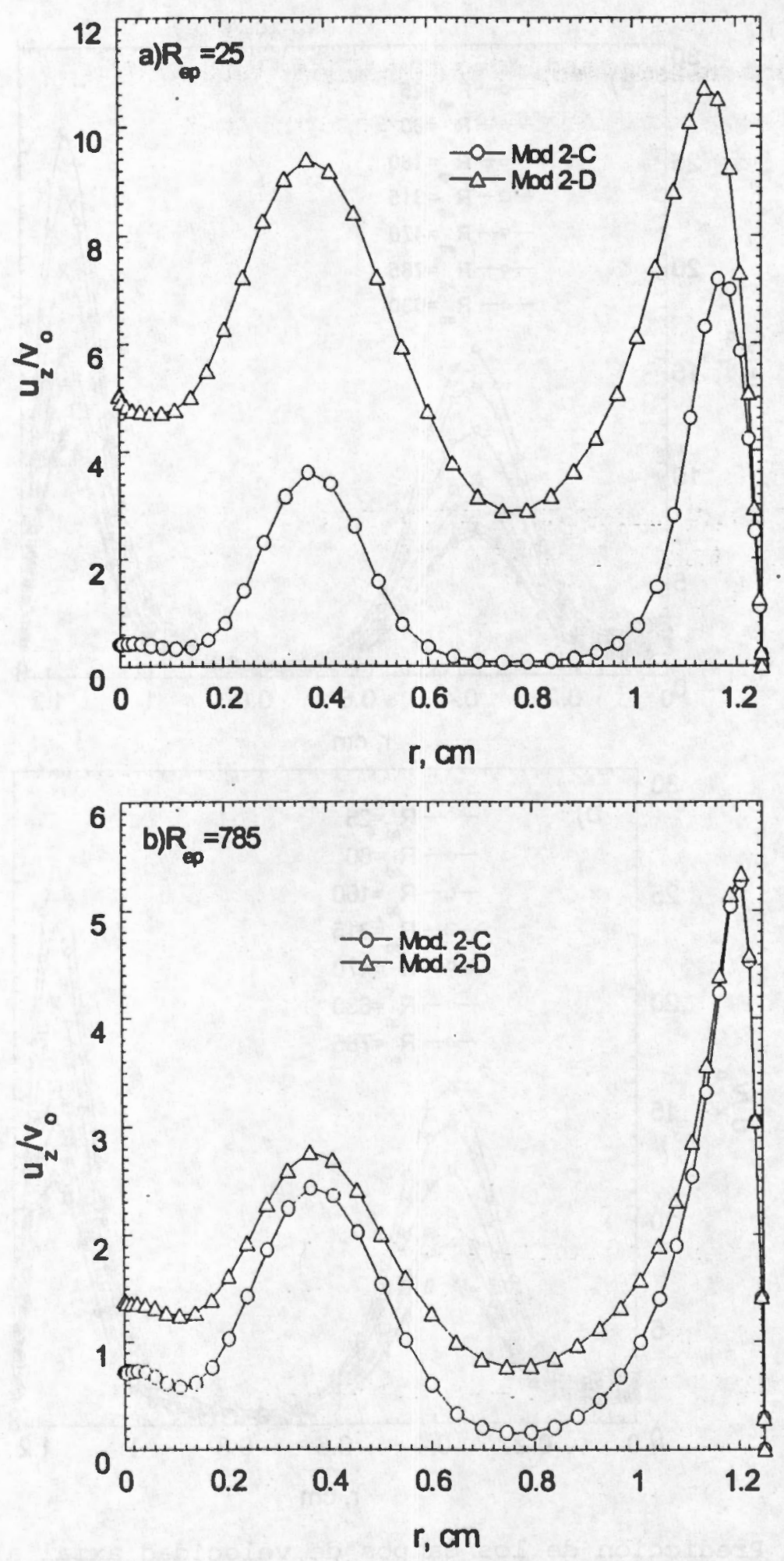

Figura 2.13. Comparación de las predicciones de los campos de velocidad axiales a lo largo del radio con el modelo 2-C y el modelo 2-D. 
Si bien la predicción del perfil de velocidad cuando se utilizó el modelo $2-E$ no se muestra en esta tesis, los resultados indican que el término viscoso axial es despreciable con respecto a los demás términos en el modelo hidrodinámico, obteniendo un perfil igual al del modelo 2C. La Figura 2.14 presenta 10 s perfiles de velocidad determinados con el modelo $2-F$. Los resultados muestran que la velocidad radial, a lo largo del radio, es cero despues de los primeros $8 \mathrm{~mm}$ del lecho, y a partir de ahi la velocidad axial es constante. Asimismo, cuando la velocidad radial es cero, los perfiles de velocidad predichos son iguales a los del modelo $2-C$. Los resultados que se tienen, muestran que la componente de la velocidad radial es insignificante con respecto a la componente de velocidad axial, comportamiento que podría deberse a que el gradiente de presión radial, utilizado para darle solución a la Ecuación (2.42), fue cero.

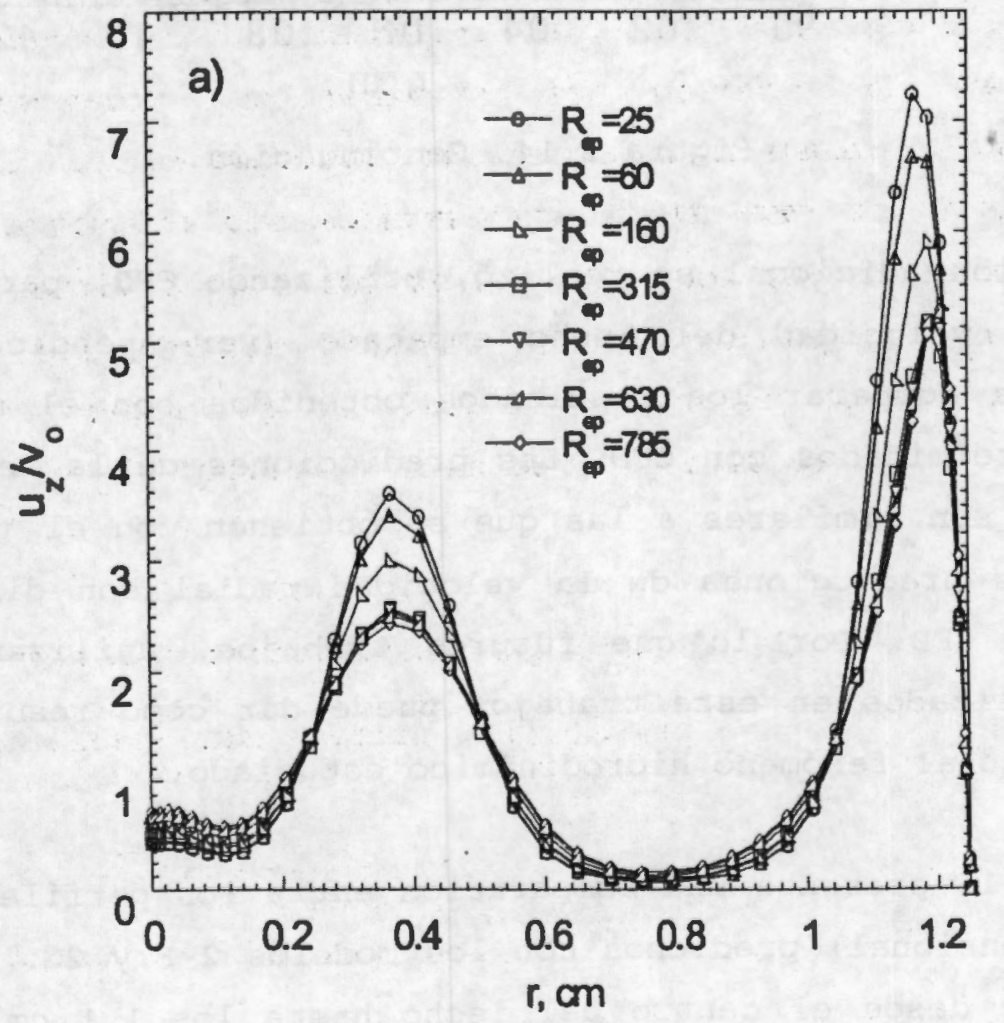

Figura 2.14. Predicciones de los campos de velocidad a lo largo del radio para el modelo $2-F$ : a) la componente axial y b) la componente radial. 


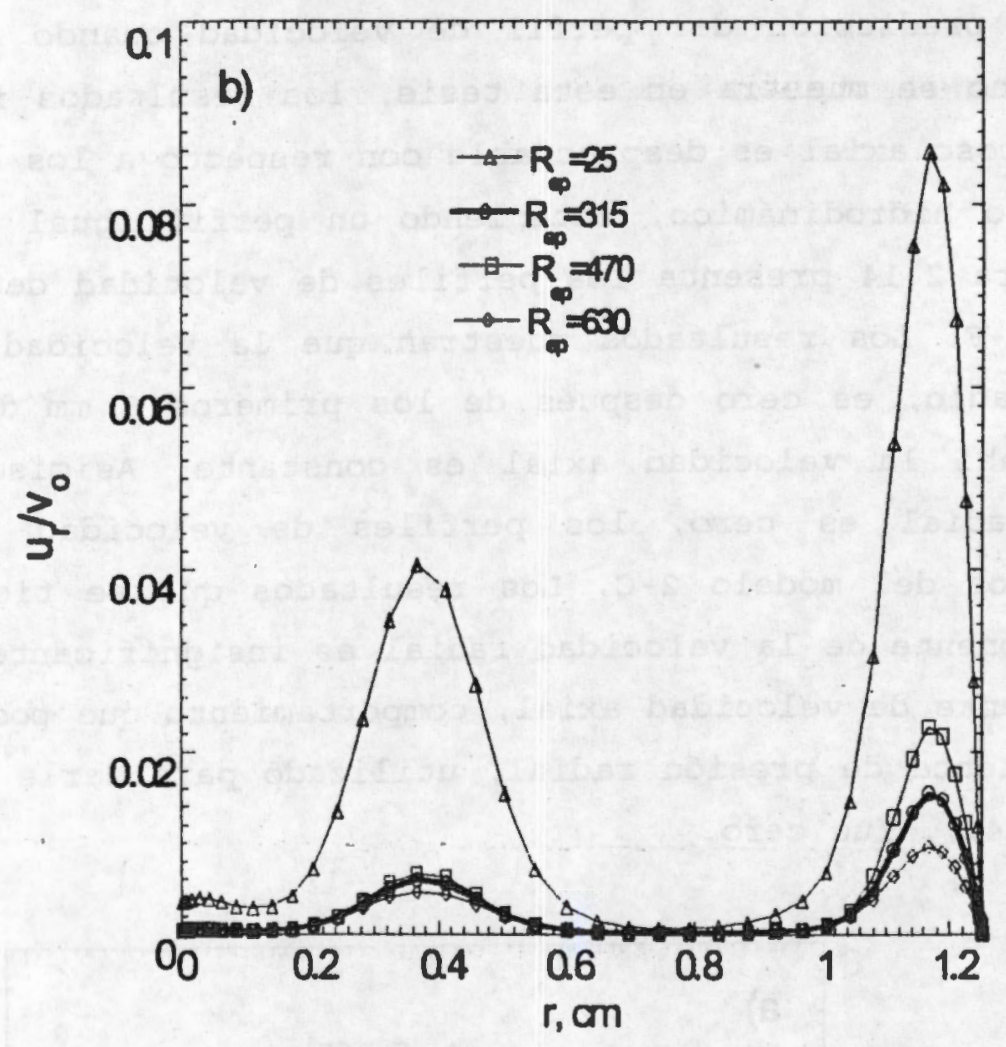

Figura 2.14. Continuación.

Una simulación adicional se realizo, utilizando CFD, para predecir los perfiles de velocidad del lecho empacado (ver Apéndice C). Esta se realizo para comparar los resultados obtenidos con el modelo $2-F$ con aquéllos determinados con CFD. Las predicciones de la velocidad axial, usando CFD, son similares a las que se obtienen con el modelo 2-F, sin embargo, las predicciones de la velocidad radial son diferentes a los que predice CFD. Por lo que futuros trabajos, utilizando CFD $Y$ los modelos utilizados en este trabajo, puede dar como resultado un mejor entendimiento al fenómeno hidrodinámico estudiado.

La Figura 2.15 presenta una comparación entre los perfiles de velocidad axial (adimensional) predichos con los modelos 2-F y 2G. Los resultados muestran que desde el centro del lecho hasta los $1.1 \mathrm{~cm}$, la velocidad obtenida con el modelo $2-\mathrm{F}$ es menor en un $10 \%$ con respecto a la determinada con el modelo 2-G. Sin embargo, desde los $1.1 \mathrm{~cm}$ hasta la pared del tubo, el modelo 2-G sobre predice la velocidad más de un $200 \%$ 
con respecto a la predicha con el modelo 2-G. Esto indica que el términos viscoso, de la ecuación de Navier-stokes, juegan un papel importante en los esfuerzos de corte que se tienen en la pared. Sin embargo en la parte anular de lecho, los términos empiricos de Darcy y Forchheimer son indispensables para considerar los fenómenos inerciales Y viscosos.

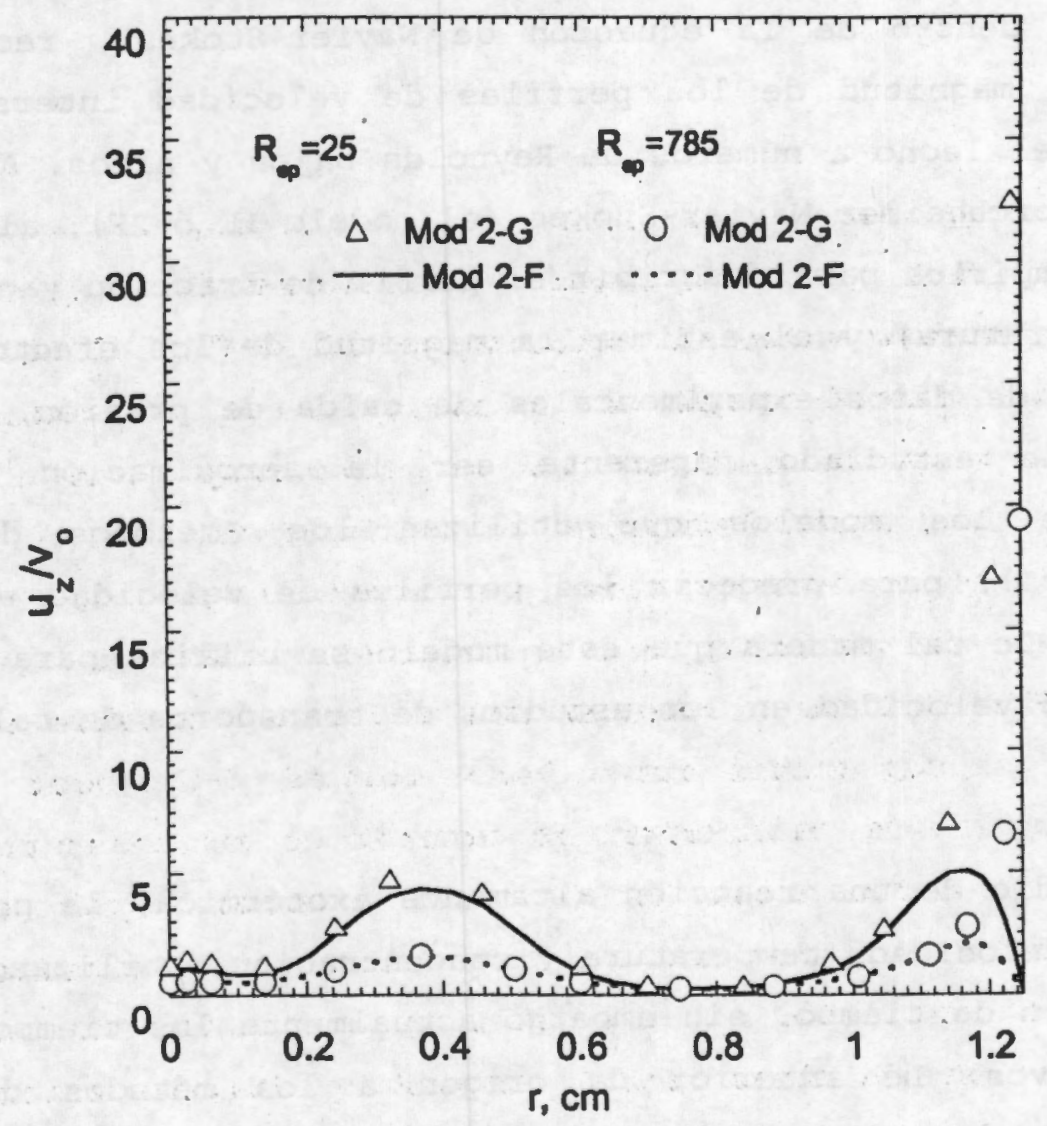

Figura 2.15. La comparación entre los modelos $2-G$ y 2-F a dos diferentes flujos.

Para tener más clạo el fenómeno hidrodinámico en un lecho empacado. En el Apendices $\mathrm{E}$ se muestra un estudio que muestra el efecto de la temperatura en la predicción de los perfiles de velocidad. Mientras que, en el Apéndice $F$ se muestra la variación de los perfiles de velocidad con el tiempo, desde el arranque hasta alcanzar el estado estacionario en el lecho. 


\subsection{Conclusiones y perspectivas}

Este estudio permitió observar las diferencias en la prediccion de lo perfiles de velocidad al utilizar la viscosidad efectiva o estimar $\alpha$ y $\beta$ de la ecuación de calda de presión de Ergun, los cuales no predicen los mismos resultados en el sistema estudiado. Los fenómenos viscosos e inerciales, considerados con los términos de Darcy y Forchheimer (acoplados dentro de la ecuación de Navier-Stokes), respectivamente, afectan la magnitud de los perfiles de velocidad intersticial en el interior del lecho a números de Reynolds bajos y altos. Asi, el modelo de Darcy-Forchheimer-Navier-Stokes (el modelo 1E 6 2F), al utilizar una ecuación empirica para describir el perfil de fracción vacía (reportada en la literatura) y al estimar la magnitud de los efectos viscosos e inerciales de datos experimentales de caida de presión, obtenidos en mismo lecho estudiado, aparenta ser la aproximación más adecuada (dentro de los modelos que utilizan los "métodos de ingenierla aproximados"), para predecir los perfiles de velocidad en el reactor estudiado. De tal manera que este modelo se utiliza para predecir los perfiles de velocidad en 10 estudios de transporte de calor sin y con reaccion.

En el estudio de una reacción altamente exotérmica, la predicción del perfil de velocidad, temperatura y concentración, utilizando CFD parece ser cuestión de tiempo, sin embargo actualmente los tiempos de cómputo son excesivos. Lo anterior da origen a los métodos de ingeniería aproximados, los cuales permiten predecir el comportamiento observado del reactor en tiempos de cómputo cortos. Además que la predicción de los perfiles de velocidad con estos métodos no fue tan alejada de aquella predicha con CFD, sin embargo, es necesario resolver algunas de sus debilidades. Por esto, en próximos estudios se deben considerar los fenómenos de turbulencia, las variaciones de la fracción vacía en las tres dimensiones, y los gradientes de presión en la dirección radial. 


\section{Capítulo 3.}

\section{Estudio del transporte de calor}

sin reacción 


\section{Estudio de trasporte de calor sin reacción}

En el presente capitulo se estudia el transporte de calor en dos lechos empacados, uno a nivel laboratorio $y$ otro a escala industrial, con similar diámetro de tubo, mismo empaque, pero diferente longitud. Ambos sistemas manejan una relación de diámetro de tubo/particula baja, $d_{e} / d_{p}-3$. El lecho a nivel laboratorio se opera de forma casi-adiabática y no-adiabática, mientras que el lecho a escala industrial solamente se opera de forma no-adiabática. El empaque utilizado es el catalizador $\mathrm{V}_{2} \mathrm{O}_{5} / \mathrm{TiO}_{2}$, empleado en el reactor industrial. En este capitulo se presenta una discusion sobre las diferentes aproximaciones que se han seguido en la literatura para estimar los parámetros de transporte de calor ( $u, k_{e z}, k_{e r} y h_{w}$ ) que son requeridos en el modelamiento del reactor a escala industrial [14].

En la sección 3.1 se presenta una breve introducción del estado del arte sobre el transporte de calor sin reacción en lechos que operan de forma casi-adiabática y no-adiabática. En la sección 3.2 se presentan los sistemas experimentales $y$ condiciones de operación utilizados en este estudio. Mientras que en la sección 3.3 se muestran los modelos que describen la situación fisica para ambas formas de operar los lechos estudiados (casi-adiabática y no-adiabática). En la sección 3.4 se discuten 108 resultados, comparando los ajustes de los perfiles de temperatura, a partir de diferentes aproximaciones utilizadas para estimar los parámetros de transporte de calor. En la sección 3.5 se presentan las conclusiones $y$ perspectivas que se tienen en el estudio de transporte de calor sin reacción.

\subsection{Transporte de calor en ausencia de reacción}

Para lograr una adecuada predicción del comportamiento observado en el reactor de oxidación parcial de o-xileno a anhidrido ftálico (u otras reacciones altamente exotérmicas; por ejemplo la oxidación de etileno) sin considerar la cinética de reacción, se necesita tener estimaciones 
- correlaciones adecuadas de los parámetros de transporte de calor y de masa que intervienen en el modelo del reactor $[14,18,19$, $27,29,30,86,87]$. Cuando se simula un reactor de lecho fijo con un modelo en dos-dimensiones (2D), se tiene que estimar $k_{\text {ez }}, k_{a x} y h_{w}$; mientras que cuando se emplea un modelo en una dimension (ID), es suficiente con estimar $U$ $y k_{a z}$. Diversas correlaciones se han reportado en la literatura para estimar los parámetros de transporte de calor. Sin embargo, la mayoría de éstas se han obtenido utilizando sistemas que manejan un amplio rango de relaciones $d_{t} / d_{p}$, dominando aquéllos que trabajan con valores de $d_{t} / d_{p} \gg 10$ [86,88-123]. Esto provoca que las correlaciones generen valores de $h_{w} y k_{t z}, k_{a x}$ alejados de los que se tienen en sistemas con $d_{t} / d_{p}<10$ y por consecuencia se tengan predicciones inadecuadas de los perfiles de temperatura observados en esta clase de reactores $[14,115]$.

Diversos estudios $[27,105,116-118]$ han sido realizados en lechos empacados que operan de forma casi-adiabática donde la dispersión axial de calor es dominante. Estos estudios utilizan normalmente un modelo pseudo-homogéneo en una dimensión para estimar la conductividad térmica efectiva axial. Si bien, hay pérdidas pequeñas de calor a través de la pared del lecho, el modelo que han utilizado es el que describe el transporte de calor en un lecho adiabático. Por esto, en el presente estudio el lecho se considera que opera en forma adiabática.

La mayoría de los estudios de transporte de calor se han llevado a cabo en lechos que operan de forma no-adiabática [14,27,90-115,119-123]. En estos se utiliza un modelo pseudo-homogéneo en $2 \mathrm{D}$ para predecir los perfiles de temperatura en la dirección axial y radial. En algunos casos se utiliza un modelo pseudo-heterogéneo $[97,98,102,104]$. Estos estudios han mostrado una relación estadistica entre $h_{w}$ y $k_{e r}$ cuando ellos se estiman del mismo conjunto de datos experimentales $[94,99,100,115]$ (ver la Figura 3.1). Esta relación estadistica se ha relacionado con los gradientes de temperatura en el interior del lecho. Es decir, dependiendo de la zona radial en el reactor en donde se tenga un gradiente temperatura mayor (que es equivalente a tener mayores 
resistencias al transporte de calor), se sabe que parámetro es estimado sin tener problemas estadísticos $[100,120]$. Por ejemplo, si el gradiente de temperatura es mayor en la región cercana a la pared del lecho, $h_{w}$ se estima con menor incertidumbre que $k_{\text {er }}$, mientras que si éste es mayor en la parte central del lecho, $k_{\text {of }}$ se estima con una mayor certidumbre que $h_{w}$.

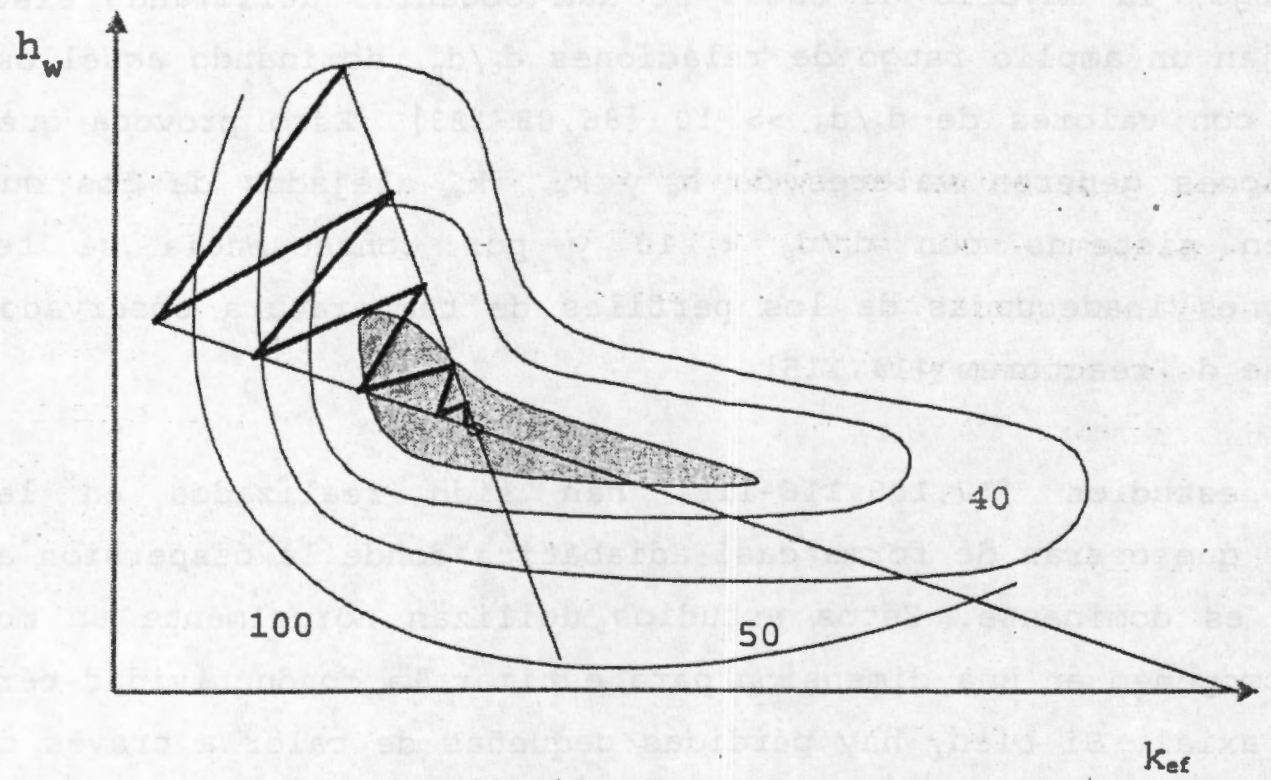

Figura 3.1. Relación estadística entre los parámetros de transporte de calor en un lecho empacado [94].

Pocos son los estudios $[27,111,112,121]$ de transporte de calor que consideran la ecuación de Navier-stokes y la distribución del empaque en el lecho para estimar $h_{w} y k_{e r}$, o unicamente $k_{e r}$ cuando el modelo hace irrelevante la existencia de $h_{w i}$. Esto es esencial cuando se tiene un lecho con una relación de $d_{c} / d_{p}$ menor a 10. En esta dirección la necesidad de considerar la hidrodinámica en la estimación de los parámetros de transporte de calor en lechos con $d_{t} / d_{p}$ menor a 10 parece ser esencial para tener éxito en la simulación del reactor industrial.

El estudio de transporte de calor sin reacción involucra dos problemas que tienen que ser resueltos en la estimación de $k_{e r} y h_{w}$. principalmente en un lecho empacado con una relación de $d_{t} / d_{p}$ baja. La 
alta correlación de $k_{\text {m }} y h_{w}$ cuando son estimados de un mismo conjunto de datos experimentales utilizando un modelo 2D; las posibles diferencias en el valor estimado de $k_{\text {ar }} y h_{w}$ cuando se considera flujo pistón o un campo de velocidad en el lecho empacado. El presente capitulo va encamino a buscar diferentes metodologís experimentales, así como evaluar diferentes estrategias teoricas para estimar con la menor incertidumbre los parámetros de transporte de calor que son utilizados en la simulación del reactor a escala industrial.

\subsection{Sistema experimental}

Las Figuras 3.2a y $3.2 \mathrm{~b}$ muestran el sistema experimental, a nivel laboratorio, utilizado en el estudio de transporte de calor sin reacción. Las características geométricas del lecho utilizado se dan en la Tabla 2.1. En ésta se resalta que la longitud del lecho de laboratorio es de $41 \mathrm{~cm}$ y se realizan dos tipos de experimentos. En el primero de ellos (ver Figura 3.2.a), se suministro calor, utilizando un foco de luz infrarroja, en el extremo superior del lecho, y se hizo pasar aire por el extremo opuesto. Esto se realizó con el propósito de medir los perfiles de temperatura a lo largo del lecho, cuando el calor se propaga en la dirección opuesta al flujo del gas. Se considera que el lecho opera en forma adiabática, ya que después de alcanzar el estado estacionario se esperaría tener un gradiente de temperatura radial despreciable. La temperatura a la entrada del lecho se mantuvo constante a $302 \mathrm{~K}$. Los perfiles axiales de temperatura se monitorearon cada 15 segundos, empleando un sistema de adquisición de datos acoplado a un sistema de cómputo. Las lecturas de temperatura se monitorearon en 10 posiciones axiales distribuidas a lo largo del eje axial del tubo, utilizando termopares (tipo $K$ ) que se colocaron en el centro del lecho. El estado estacionario se alcanzó en alrededor de una hora desde el arranque del experimento. Esto se observó cuando ya no hubo cambio en el perfil de temperatura. En la Tabla 3.12 se dan los números de Reynolds de particula $\left(R e_{p}\right)$ que se utilizaron en este experimento. La 
corrida 6 y 10 corresponden a los $\left(R e_{p}\right)$ que se utilizan en un reactor industrial.

Tabla 3.I. Condiciones experimentales

\begin{tabular}{cccc}
\hline $\begin{array}{l}\text { Los experimentos } \\
\text { axiales }\end{array}$ & \multicolumn{2}{c}{$\begin{array}{c}\text { Los experimentos } \\
\text { axiales y radiales. }\end{array}$} \\
\hline Corrida & $\mathrm{Re}_{\mathrm{p}}$ & Corrida & $\mathrm{Re}_{\mathrm{p}}$ \\
1 & -0 & 7 & 25 \\
2 & 80 & 8 & 50 \\
3 & 190 & 9 & 160 \\
4 & 360 & 10 & 630 \\
5 & 510 & & \\
6 & 630 & & \\
\hline
\end{tabular}

En el segundo tipo de experimentos (ver Figura $3.2 \mathrm{~b}$ ) se utilizó el mismo lecho empacado, pero en esta ocasión éste se opero en forma noadiabática.. El. lecho se calentó a través de la pared con una resistencia eléctrica. La temperatura de la pared del lecho se mantuvo alrededor de $673 \mathrm{~K}$, teniendo el punto de control de ésta a la mitad de la longitud total del lecho.

a)

$$
\text { Lampara Infrarroja }
$$

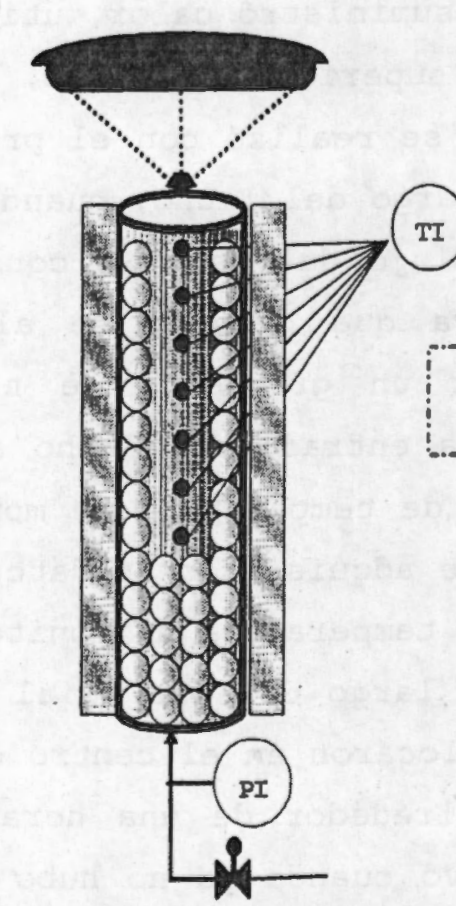

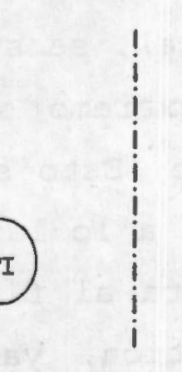

Lectura de Temperatura

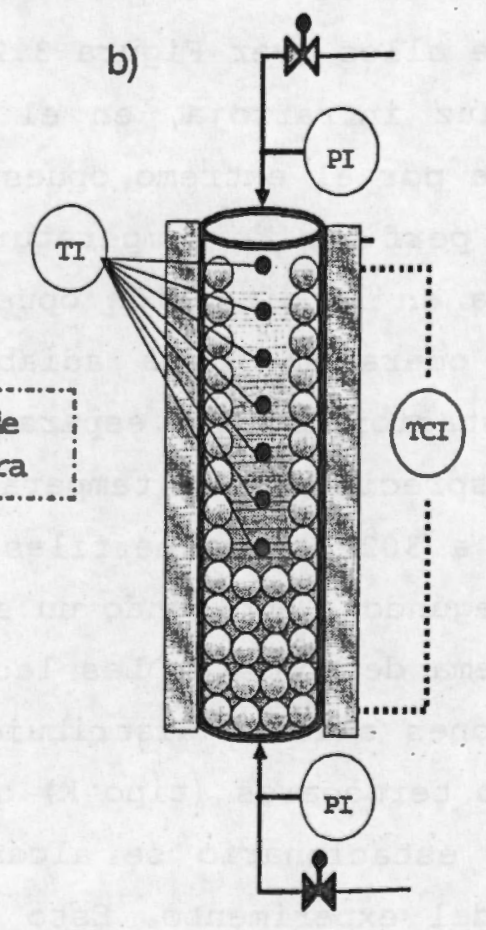

Figura 3.2. Sistemas experimentales usados en el estudio de transporte de calor en el lecho de laboratorio. a) Adiabatico; b) no-adiabático. 
Esta temperatura se eligio porque es la misma que se utiliza en la operación de un reactor industrial, cuyo calentamiento es con un baño de sal [14]. La temperatura en el interior del lecho se monitoreó en las dixecciones axial y radial, y el estado estacionario se alcanzó en aproximadamente una hora. En la Tabla $3.1 \mathrm{~b}$ se muestran los $\mathrm{Re}_{\mathrm{p}}$ que fueron utilizados en la operación del lecho de forma no-adiabatica. Asimismo, en este estudio se contó con los perfiles de temperatura observados, sin reacción en un lecho a escala industrial operado de forma no-adiabática [14], el cual presenta una relación de $d_{t} / d_{p}$ $=3.097$, una fracción vacía promedio igual a 0.5 y una longitud de 250 $\mathrm{Cm}$. La Figura 3.3 muestra el arreglo de los termopozos que se soldaron en la pared del tubo para introducir los termopares. Este arreglo permitió monitorear la temperatura a lo largo de la posición axial y radial en el lecho. La misma configuración de los termopozostermopares se tenia en el lecho de escala laboratorio e industrial. Más detalles sobre los sistemas experimentales en ambos lechos se puede revisar en los trabajos de Castillo-Araiza y Lopez-Isunza $[14,38]$.

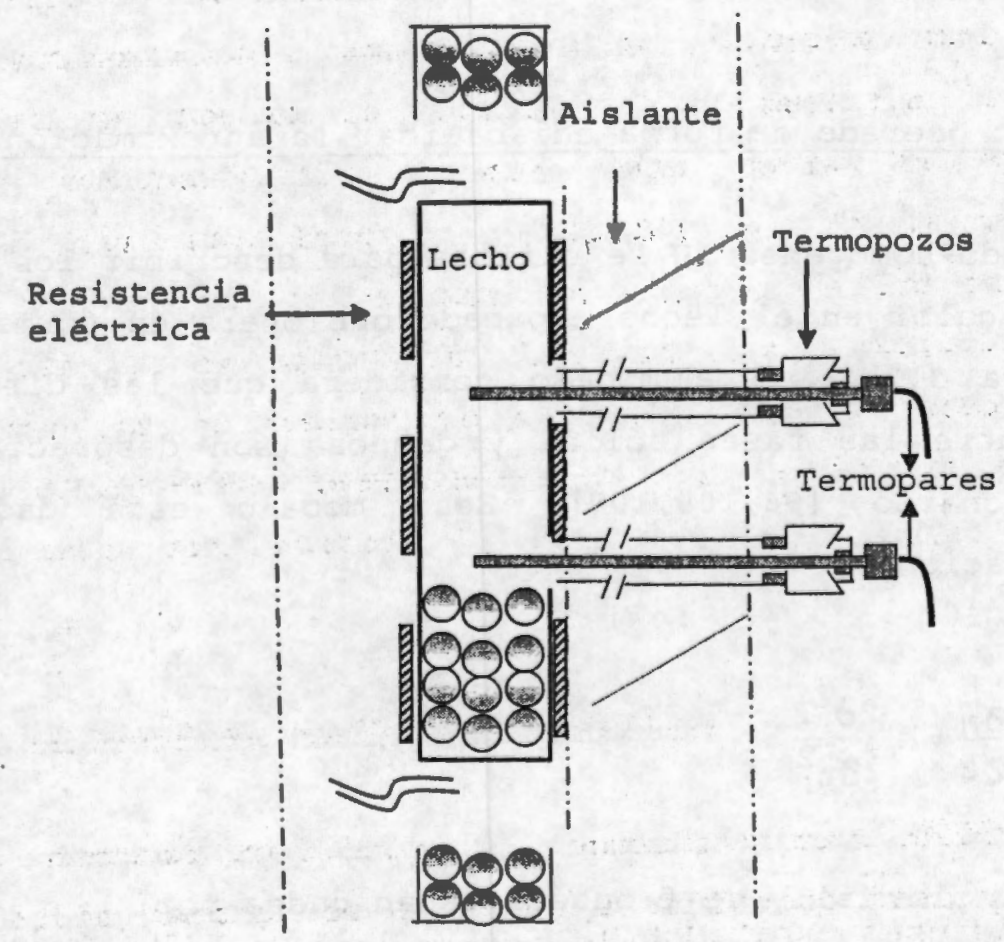

Figura 3.3. Arreglo de los termopares y termopozos a 10 largo del lecho. 


\subsection{Modelos de transporte de calor en el lecho empacado}

En esta sección se presentan los modelos que describen el comportamiento fenomenológico del lecho, cuando éste se opera de forma adiabática y no-adiabática (ver la Figura 3.2). Estos modelos, en conjunto con los datos experimentales obtenidos en cada tipo.de experimento, se emplean en la estimación de los parámetros de transporte de calor efectivos. El desarrollo de estos modelos se baso en lo siguiente:

1. -El fluido es newtoniano.

2. Las propiedades físicas del fluido, viscosidad $\left(\mu_{f}\right)$, calor especifico $\left(C_{p t}\right)$ y densidad $\left(\rho_{\varepsilon}\right)$, son constantes.

3. -El modelo hidrodinámico, cuando éste es utilizado, considera únicamente la componente de velocidad axial, como una función del radio y la longitud del lecho. La componente de velocidad radial se considera despreciable (modelo $1-E$, ver el capitulo 2 ).

\subsubsection{El lecho operado de forma adiabática: la aproximación clásica}

El modelo pseudo-homogéneo $1 \mathrm{D}$ se utiliza para describir los procesos de transporte de calor en el lecho empacado que opera en forma adiabatica (ver la Figura 3.2b). Este modelo considera que las diferencias de temperatura entre las fases sólida y gaseosa son despreciables en el estado estacionario $[98,105,107]$. Este modelo está dado por las siguientes ecuaciones:

$\rho_{s} C_{p=} \frac{\partial \bar{z}}{\partial t}+v_{o} \rho_{z} C_{p t} \frac{\partial \dot{z}}{\partial z}=k_{e z} \frac{\partial^{2} T}{\partial z^{2}}$

Las condiciones inicial y de frontera están dadas por:

$t=0 ; \quad T(t, z)=T_{s s}$ 


$$
\begin{array}{ll}
z=0 ; & v_{z o} \rho_{f} C_{p t}\left(T_{0}-T\right)=-k_{e z} \frac{\partial T}{\partial z} \\
z=L ; & -k_{e z} \frac{\partial T}{\partial z}=q_{0}
\end{array}
$$

Donde $q_{0}$ es el flujo de calor suministrado por la lámpara en la parte superior del lecho, $y$ vo es el flujo volumetrico dividido entre la área transversal del lecho.

Las ecuaciones anteriores en forma adimensional toman la siguiente forma :

$w \frac{\partial Y}{\partial \tau}=\operatorname{Re}_{1}\left(\frac{1}{\operatorname{Pe}_{z}} \frac{\partial^{2} Y}{\partial \zeta^{2}}-\frac{\partial Y}{\partial \zeta}\right)$

La cual está sujeta a las siguientes condiciones, inicial y de frontera adimensionales :

Tiempo adimensional:

$\tau=0 ; \quad Y(\tau, \zeta)=Y_{s}$

En la posición axial adimensional:

En $\zeta=0 ; \quad(Y-1)=\frac{1}{\mathrm{Pe}_{z} V_{z}} \frac{\partial Y}{\partial \zeta}$

En $\zeta=1 ; \quad \frac{\partial Y}{\partial \zeta}=\mathrm{Pe}_{2} \mathrm{Q}_{0}$

\subsubsection{El lecho operado de forma no-adiabática: la aproximación clásica}

En esta sección se presentan los modelos que se emplean para describir los procesos de transporte de calor en ambos lechos, a escala laboratorio e industrial, cuando éstos operan de forma no-adiabática. Un primer modelo considera que $k_{a x} y h_{w}$ se agrupan dentro de un 
coeficiente global de transporte de calor U [124], esto se logra cuando se aplica colocación ortogonal, un punto de colocación ortogonal en la dirección radial, al modelo en $2 \mathrm{D}$, teniendo asi un modelo en ID espacial. El segundo modelo utiliza $k_{a x}, k_{\text {er }} y h_{w}, y$ un modelo en 2D. En ambos modelos se considera que las diferencias de temperatura entre las fases sólida y gaseosa son despreciables.

\subsubsection{Modelo en 1D espacial}

Finlayson (1971) [125] desarrolla dos expresiones para U, aplicando colocación ortogonal con un sólo punto de colocación en el modelo de transporte de calor en 2D. Estas expresiones anteriormente fueron encontradas por Beek Y Singer (1951) [123], promediando el perfil radial de temperatura en el lecho. El modelo en $1 D$, que es el resultado de aplicar colocación ortogonal al modelo en $2 \mathrm{D}$, que utiliza el coeficiente global de transporte de calor $U$ y considera la dispersión axial de calor, es:

$\rho_{s} C_{p s} \frac{\partial T}{\partial t}+v_{z o} \rho_{z} C_{p l} \frac{\partial T}{\partial z}=k_{e z} \frac{\partial^{2} T}{\partial z^{2}}+\frac{4 U}{d t}(T-T)$

Las correspondientes condiciones inicial y de frontera son:

$t=0 ; \quad T(t, z)=T_{s,}$

$z=0 ; \quad v_{z o} \rho_{f} C_{p t}\left(T_{o}-T\right)=-k_{e z} \frac{\partial T}{\partial z}$

$z=L_{i} \quad \frac{\partial T}{\partial z}=0$

Estas ecuaciones en forma adimensional son:

$W \frac{\partial Y}{\partial \tau}=\operatorname{Re}_{s}\left(\frac{1}{P e_{z}} \frac{\partial^{\prime} Y}{\partial \zeta^{2}}-\frac{\partial Y}{\partial \zeta}+A\left(Y_{w}-Y\right)\right)$ 
Condición inicial:

$\tau(0, \zeta)=0, \quad Y=Y_{a}$

Las condiciones de frontera son:

En $\zeta=0 ; \quad(Y-1)=\frac{1}{\mathrm{Pe}_{z} \mathrm{~V}_{2}} \frac{\partial Y}{\partial \zeta}$

$\operatorname{En} \zeta=1 ; \quad \frac{\partial y}{\partial \zeta}=0$

\subsubsection{Modelo en 2D espaciales}

El modelo en 2D considera, además del transporte de calor por convección, la dispersión axial y radial de calor. Este modelo también podría utilizarse en la descripción del transporte de calor en el lecho operado de forma adiabatica, utilizando su respectiva condición de frontera en la pared del tubo. Este modelo esta dado por las siguientes ecuaciones :

$\rho_{s} C_{p z} \frac{\partial T}{\partial t}+v_{z} \rho_{z} C_{p \varepsilon} \frac{\partial T}{\partial z}+v_{r} \rho_{z} C_{p t} \frac{\partial T}{\partial r}=k_{a x}\left(\frac{\partial^{2} T}{\partial x^{2}}+\frac{1}{r} \frac{\partial T}{\partial r}\right)+k_{e x} \frac{\partial^{2} T}{\partial z^{2}}$

Las condiciones, inicial $y$ de frontera, que representan la situación fisica del sistema, ver la Figura $3.2 \mathrm{~b}$, son:

Condición inicial:

$t(0, r, z)=0 ; \quad T=T_{E s}$

Condiciones de frontera:

Posición axial:

En $z=0 ; \quad v_{z}(r) \rho_{q} C_{p s}\left(T-T_{0}\right)=k_{a x} \frac{\partial T}{\partial z}$ 
En $z=L ; \quad \frac{\partial T}{\partial z}=0$

Posición radial:

En $r=0 ; \quad \frac{\partial T}{\partial r}=0$

Donde $v_{2} \quad y \quad v_{r}$ son las componentes de la velocidad axial $y$ radial respectivamente. Cuando toma lugar la aproximación clásica: $v_{r}=0$ y $v_{z}$ es el flujo volumétrico dividido entre la área transversal del lecho ( $\left.v_{0}\right)$. Para este modelo tres posibles condiciones de frontera pueden ser utilizadas en la pared del lecho:

Para la aproximación clásica, la condición de frontera es:

En $r=R_{T i} \quad-k_{\text {er }} \frac{\partial T}{\partial r}=h_{w}\left(T-T_{w}\right)$

La condición de frontera al considerar el modelo hidrodinámico es:

En $r=R_{T} ; \quad T=T_{w}(z)$

En donde $T_{w}(z)$, es la temperatura en la pared interna, a diferentes posiciones axiales, del lecho. Los datos experimentales de $T_{w}$ se ajustaron a un polinomio, el cual se utiliza en la solución numérica del modelo. Si se utiliza la temperatura del baño de enfriamiento ( $T_{b}$ ), en lugar de $T_{w}(z)$, los ajustes de temperatura están alejadas de las observadas.

La condición de frontera en la pared del lecho, cuando se opera el lecho de forma adiabática, es:

En $r=R_{T} ; \quad \frac{\partial T}{\partial r}=0$ 
Cuando el modelo hidrodinámico se acopla al de transporte de calor, y únicamente se conoce la $\mathrm{T}_{\mathrm{b}}$, puede emplearse la aproximación de la capa IImite, la cual aplica cuando la velocidad radial es cero. En este caso se utiliza la condicion de frontera (3.21a) y $T_{w}=T_{b}$. Diversos trabajos $[61,109,112,121]$, que acoplan la hidrodinámica al modelo de transporte de calor, utilizan la condición de frontera (21a), sin embargo, no es claro si aplican la aproximación de la capa limite.

Las ecuaciones anteriores, al definir algunas variables adimensionales, $(3.17-3.21)$ toman la siguiente forma:

$W \frac{\partial Y}{\partial \tau}=\frac{R_{e}}{P e}\left[\frac{1}{\xi} \frac{\partial Y}{\partial \xi}+\frac{\partial^{2} Y}{\partial \xi^{2}}\right]+\frac{R_{e}}{P e_{2}} \frac{\partial^{2} Y}{\partial \zeta^{2}}-R_{e s} V_{z}(\xi, \zeta) \frac{\partial Y}{\partial \zeta}-R_{e} V_{r}(\xi, \zeta) \frac{\partial Y}{\partial \xi}$

Condición inicial:

Al $\tau=0, \quad Y=Y_{s:}$

Las condiciones de frontera son:

Posición axial adimensional:

En $\zeta=0 ; \quad(Y-1)=\frac{1}{\operatorname{Pe}_{2} V_{2}(\xi, \zeta)} \frac{\partial Y}{\partial \zeta}$

$\operatorname{En} \zeta=1 ; \quad \frac{\partial Y}{\partial \zeta}=0$

Posición radial adimensional:

En $\xi=0 ; \quad \frac{\partial y}{\partial \xi}=0$

En $\xi=1 ; \quad \frac{\partial Y}{\partial \xi}=B i_{v}\left(Y_{w}(\zeta)-Y\right)$

$$
Y=Y_{w}(\zeta)
$$

$$
\frac{\partial Y}{\partial \xi}=0
$$




\subsection{Resultados y discusión sobre el estudio de transporte de calor}

En la sección 3.4 .1 se ajustan los perfiles de temperatura observados, a diferentes números de Reynolds, al operar el lecho de laboratorio de forma adiabática (ver Figura 3.2a). El ajuste de estas observaciones al utilizar las ecuaciones $(3.1-3.4)$ sirvio para estimar la $k_{02}, y$ asf proponer una correlación modificada (de Yagi-Kunii-Wakao (YKW); dada por la Ecuación (3.30)), que da el valor del número de Peclet en términos del número de Reynolds. Esta correlación se emplea para conocer el valor de la conductividad térmica efectiva que se utiliza en el modelo en 2D, cuando se consideran, $\sigma$ no, los perfiles de fracción vacia y los campos velocidad en el interior del lecho.

En la sección 3.4 .2 se discute el comportamiento observado en ambos lechos, de laboratorio y a escala industrial, cuando estos se operan de forma no-adiabática. Mientras que en la sección 3.4 .3 se discute el ajuste de los perfiles de temperatura de ambos lechos cuando se consideran dos casos y flujo pistón en éstos. En el primero se estima $U$, empleando el modelo pseudo-homogéneo en 1D (ver las ecuaciones (3.93.12)), que se obtiene al aplicar colocacion ortogonal al modelo en 2D. En el segundo se estiman $h_{w} y k_{a r}$ utilizando el modelo pseudo-homogéneo en 2D (ver las ecuaciones (3.17-3.21a)). Finalmente, en la sección 3.4 .4 se estiman $h_{w} y k_{a r}$ al considerar la hidrodinámica $y$ el perfil de fracción vacía en el lecho.

\subsubsection{Transferencia de calor axial: la operación adiabática del lecho}

La Figura 3.4 muestra una comparación entre los perfiles de temperatura observados y los ajustados a diferentes números de Reynold Re (Tabla 3.1; corridas 1-6). Las diferencias entre éstas son alrededor de 1-3 k, dependiendo del flujo de alimentacion. Esta Figura muestra que el perfil de temperatura, en ausencia de flujo de aire, presenta los mayores gradientes de temperaturas, mostrando la contribución de las conductividades moleculares del sólido y el gas estancado en el lecho. 
Asimismo, conforme el flujo de aire se incrementa estos gradientes de temperatura disminuyen debido al mayor arrastre de calor en el lecho.

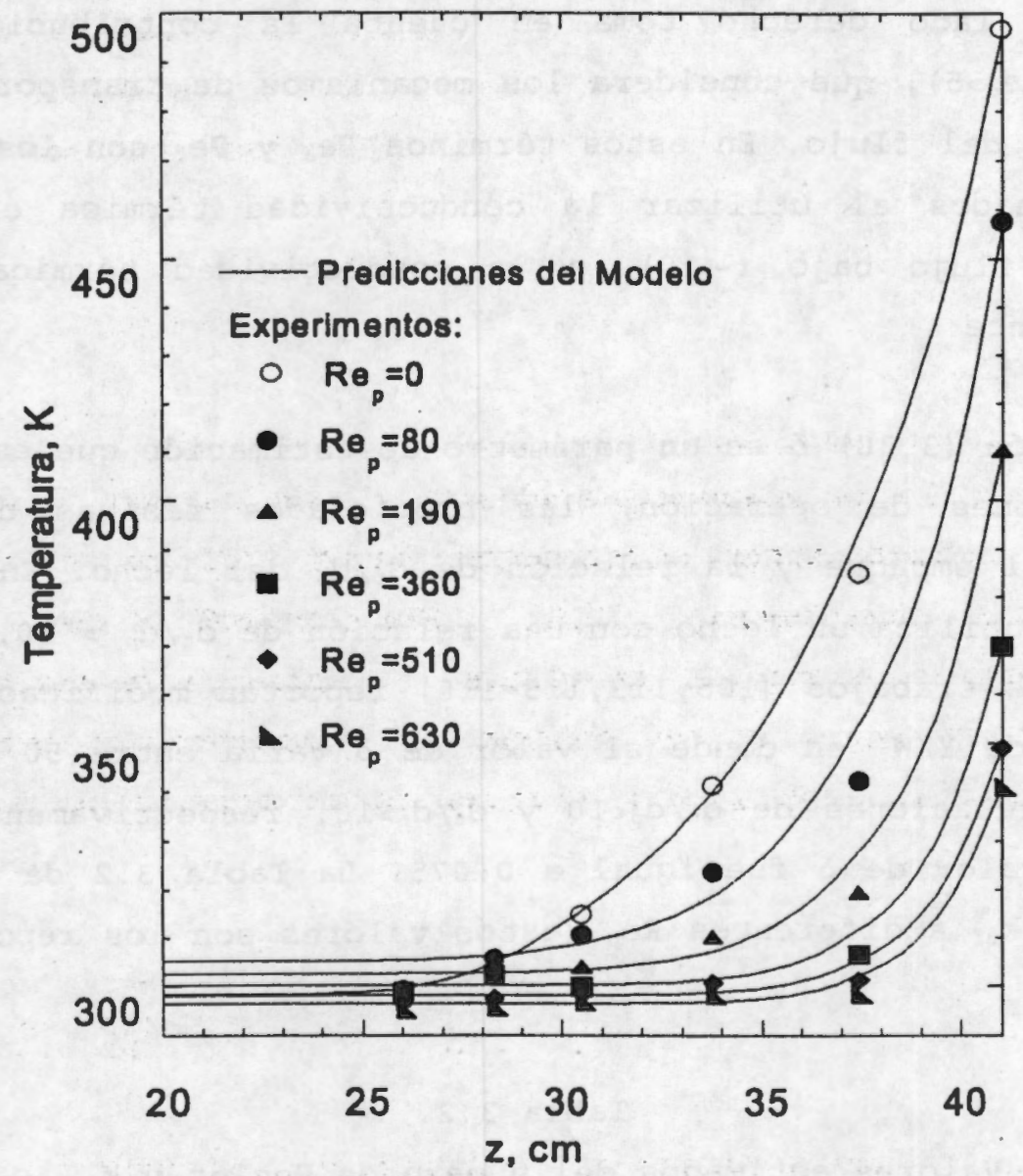

Figura 3.4. Comparación entre los perfiles de temperatura experimental $y$ ajustado en la dirección axial al estimar la $k_{e x}$.

Los valores estimados de la conductividad térmica efectiva $k_{a z}$, a diferentes flujos de alimentación, se pueden expresar en términos del número de Reynolds de particula, como lo hizo YKW [116], quienes proponen la siguiente correlación:

$\frac{1}{\mathrm{Pe}}=\frac{1}{\mathrm{Pe}^{0}}+\frac{\delta}{\mathrm{Pe}_{\mathrm{f}}} \mathrm{Re}_{\mathrm{p}} \mathrm{P}_{\mathrm{r}}$ 
El primer termino del lado derecho de esta ecuación considera la contribución estática (cuando el $\mathrm{Re}_{\mathrm{p}}<5$ ), que toma en cuenta los mecanismos de transporte de calor independientes del flujo; el segundo término del lado derecho toma en cuenta la contribución dinámica (cuando el $\mathrm{Re}_{\mathrm{p}}>5$ ), que considera los mecanismos de transporte de calor dependientes del flujo. En estos términos $\mathrm{Pe}_{\circ}$ y $\mathrm{Pe}_{t}$ son los números de Peclet evaluados al utilizar la conductividad térmica efectiva del lecho a un flujo bajo ( 0$)$, y la conductividad térmica del aire, respectivamente.

En la ecuación (3.30) $\delta$ es un parámetro de estimación que es función de las condiciones de operación, las propiedades físicas del gas, la geometría del empaque y la relación de $d_{\varepsilon} / d_{p}$ del lecho. En el estudio de YKW, que utiliza un lecho con una relación de $d_{k} / d_{p}>10, \delta$ es igual 0.8 . Diversos trabajos $[105,113,115-118]$ reportan modificaciones de la correlación de $\mathrm{YKW}$, en donde el valor de $\delta$ varía entre 50 y $300 \%$ para lechos con relaciones de $d_{c} / d_{p}<10$ y $d_{c} / d_{p}>10$, respectivamente. En este estudio el valor de $\delta$ fue igual a 0.075 . La Tabla 3.2 da los valores del Pe y la ket a diferentes $R_{p}$ lestos valores son los reportados para el caso A).

Tabla 3.2.

\begin{tabular}{ccc} 
Valores estimados del nümero de Peclet $y k_{e z}$ \\
\hline $\operatorname{Re}_{\mathrm{p}}$ & Pe & $k_{\text {ez }}$ J. (m.s.K) $)^{1}$ \\
\hline 25 & 4.3 & 0.36 \\
60 & 5.1 & 0.60 \\
160 & 7.5 & 1.31 \\
630 & 15.5 & 2.47 \\
\hline
\end{tabular}

La Figura 3.5 compara los valores estimados (en este estudio) del número de Peclet, a diferentes números de Reynolds de particula, con los predichos al utilizar diferentes modificaciones de la correlación de YKW (ya reportadas en la literatura $[105,113,115-118]$ ), y la Ecuación (3.30) utilizando el valor modificado de $\delta(0.075)$. La 
correlación de YKW fue desarrollada utilizando sistemas con una relación de $d_{\varepsilon} / d_{p}$ mayor a 10 . Mientras que las correlaciones modificas de YKW propuestas por Bey Y Eigenberger (2001) y Demirel et al. (2000) fueron utilizadas en un sistema con una relación de $d_{c} / d_{p}$ menor a 10 . Caracteristicas de estas correlaciones $y$ otras se puede revisar en el trabajo de Castillo-Araiza $y$ Went et al. $[38,115]$. Esta comparación muestra la necesidad de tener estimaciones independientes del Pe, ya que de lo contrario las diferencias son considerables. Los resultados anteriores muestran que el parámetro $\delta$ considera el impacto de los campos de velocidad (por la alta fracción vacía) en los procesos de transporte de calor en el lecho. La Figura 3.5b muestra la línea de paridad del Pe donde se muestra que si no estima el Pe de forma independiente en el sistema estudiado las desviaciones son importantes.

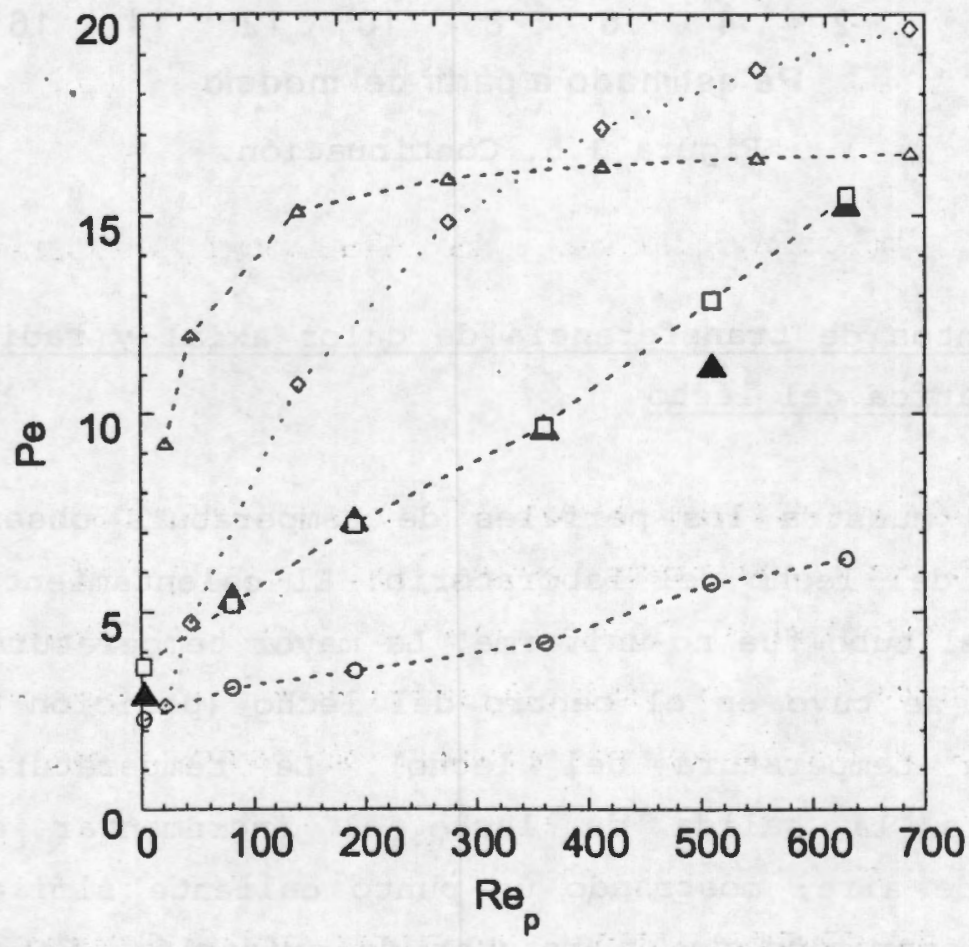

Figura 3.5. a) Dependencia del Pe con el número de Reynolds $R_{p}$. b) Linea de paridad. (A) Ajuste, ( $\square$ ) correlación modificada de YKW, (O) correlación de YKW (1960), ( $\Delta$ ) Bey y Eigenberger (2001) y (0) Demirel et al. $(2000)$. 


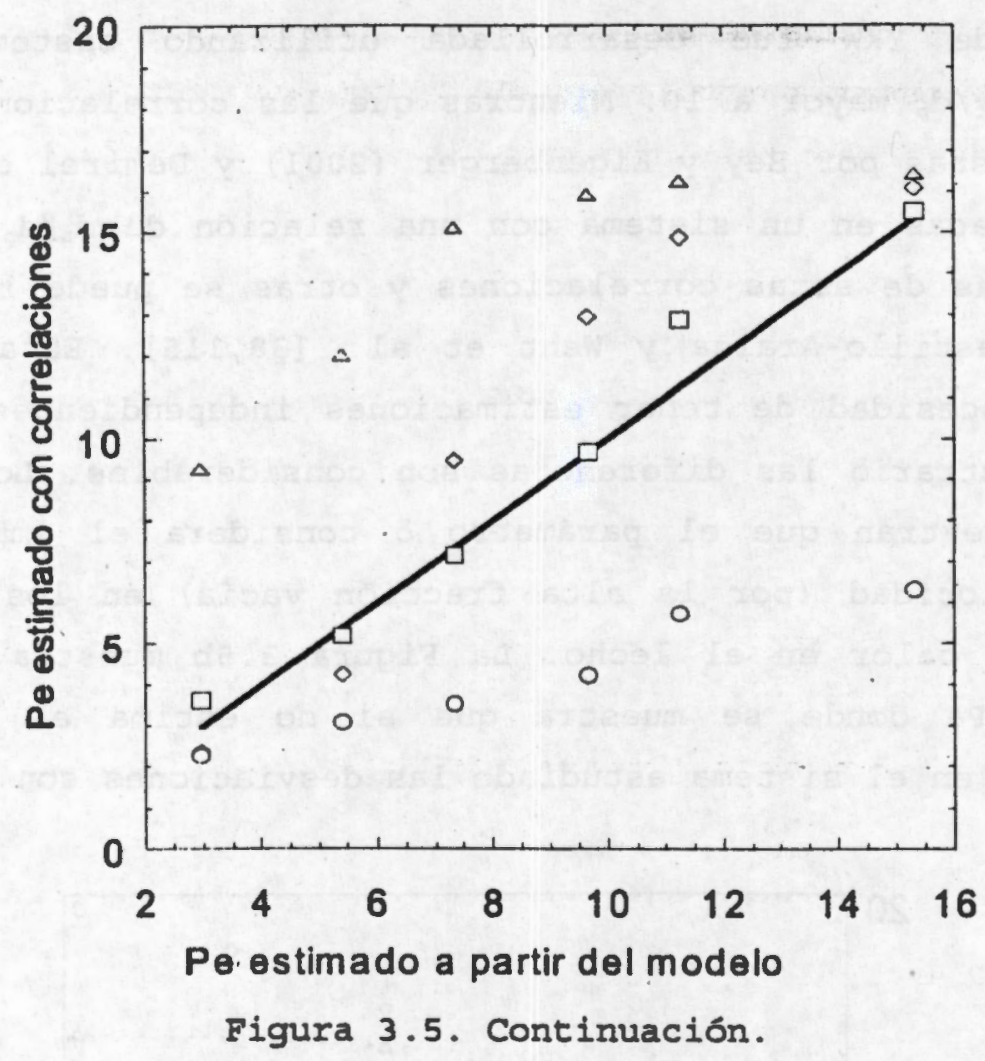

3.4.2 Experimentos de transferencia de calor axial y radial: operación no-adiabática del lecho

La Figura 3.6 muestra los perfiles de temperatura observados en la pared interna del lecho del laboratorio. El calentamiento a lo largo de la pared del tubo fue no-uniforme. Ia mayor temperatura, a un flujo igual a cero, se tuvo en el centro del lecho (posición en la que se controlaba la temperatura del lechol. La temperatura máxima se desplazo hacia la salida de lecho al incrementar el flujo de alimentación de aire; mostrando un punto caliente similar al que se tienen en la operación de un reactor de oxidación. Estos resultados muestran que el calentamiento del lecho empacado es deficiente. 


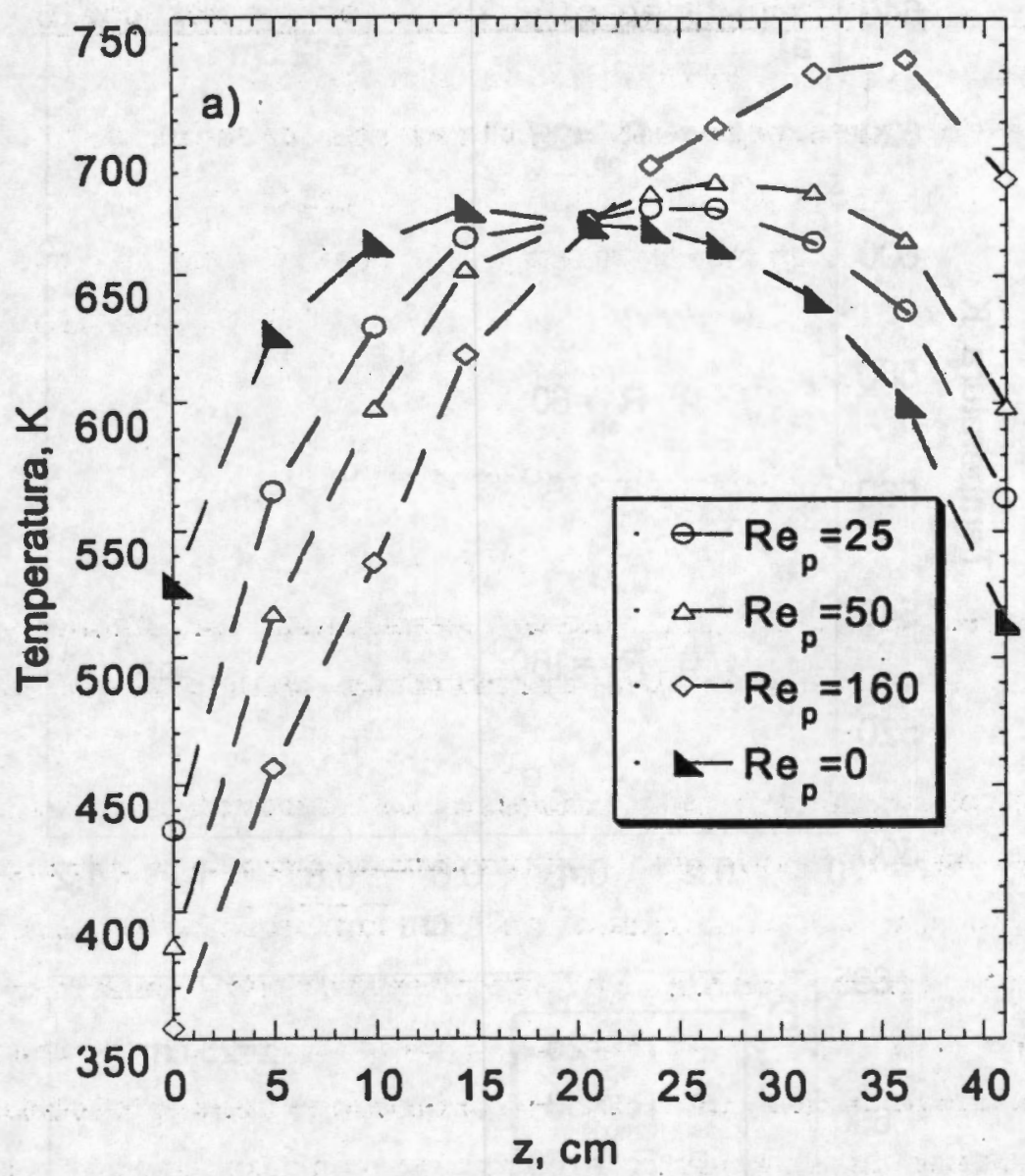

Figura 3.6. Perfiles de temperatura experimentales en la pared interna del lecho a diferentes números de $R_{p}$.

La Figura 3.7 muestra los perfiles de temperatura radiales observados en el lecho, a diferentes posiciones axiales $y \operatorname{Re}_{p}$, para las corridas 7-9 (ver la Tabla 3.1). En ésta se observa que, a una distancia de $2 \mathrm{~mm}$ de la pared del reactor, los gradientes de temperatura son alrededor de 10-30 $\mathrm{K}$ (que es un 20-50\% del gradiente total), dependiendo de la posición axial y flujo que se maneje. Esto indica la presencia de importantes resistencias de transporte de calor en esta región. Esta calda de temperatura se podría deber al impacto de la fracción vacia en esta región. 

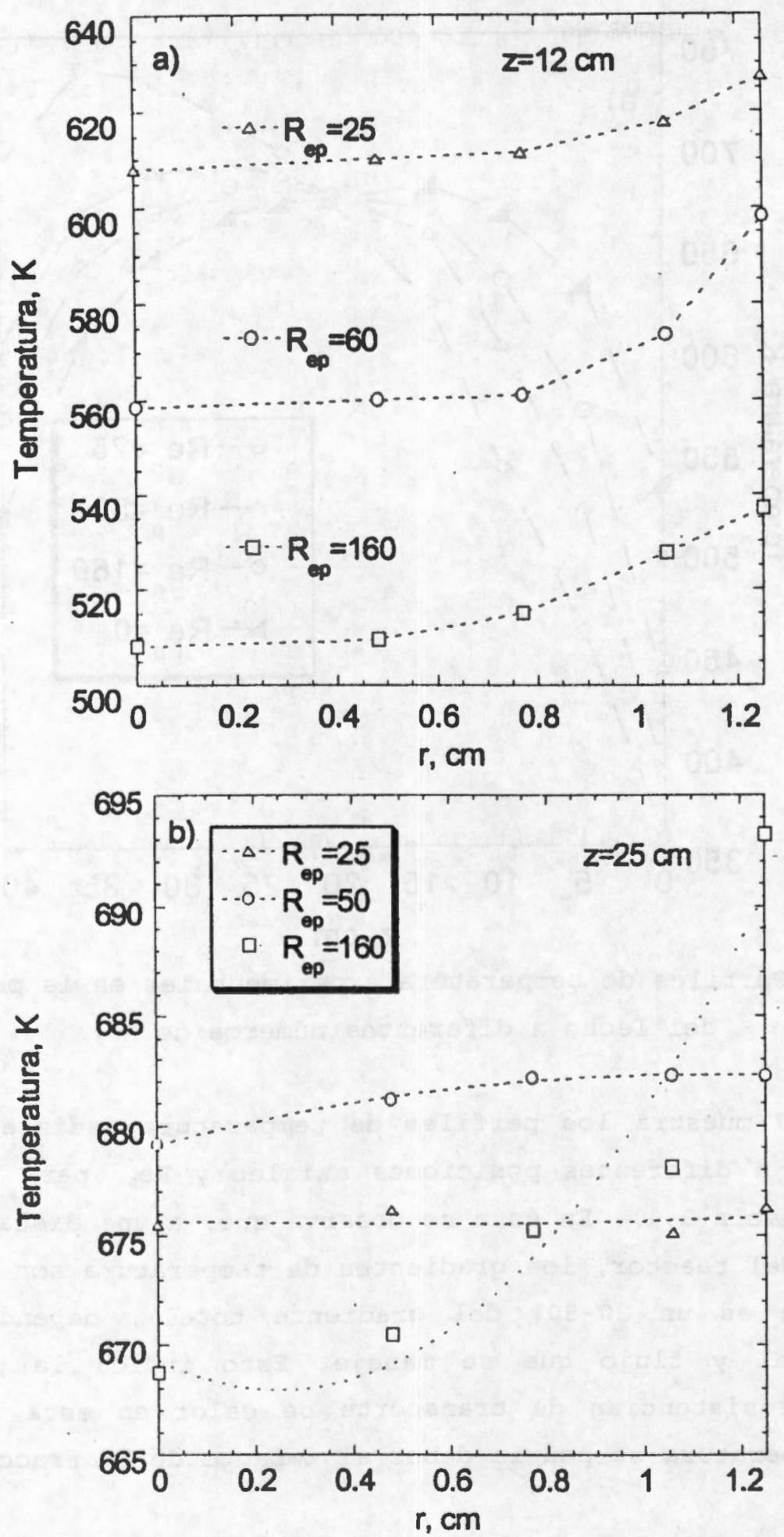

Figura 3.7. Perfil de temperatura radial en función del $\operatorname{Re}_{\mathrm{p}} \mathbf{a}$ diferentes posiciones axiales del lecho. a) $z=12 \mathrm{~cm}$ y b) $z=25 \mathrm{~cm}$. 
La Figura 3.8 muestra los perfiles de temperatura observados, en la dirección radial, a diferentes posiciones axiales del lecho a escala industrial [14]. Ya que la relación de $d_{r} / d_{p}$ de este reactor es similar a la del lecho de laboratorio, el transporte de momento afecta al de calor de la misma manera. Sin embargo, los perfiles de temperatura radiales en el lecho a escala industrial, a diferencia del lecho de laboratorio, no son simétricos. Esto se debe a que los termopozostermopares fueron dispositivos que actuaron como medio de transporte de calor desde el exterior hasta el interior del lecho o viceversa (dependiendo si habia reacción o no).

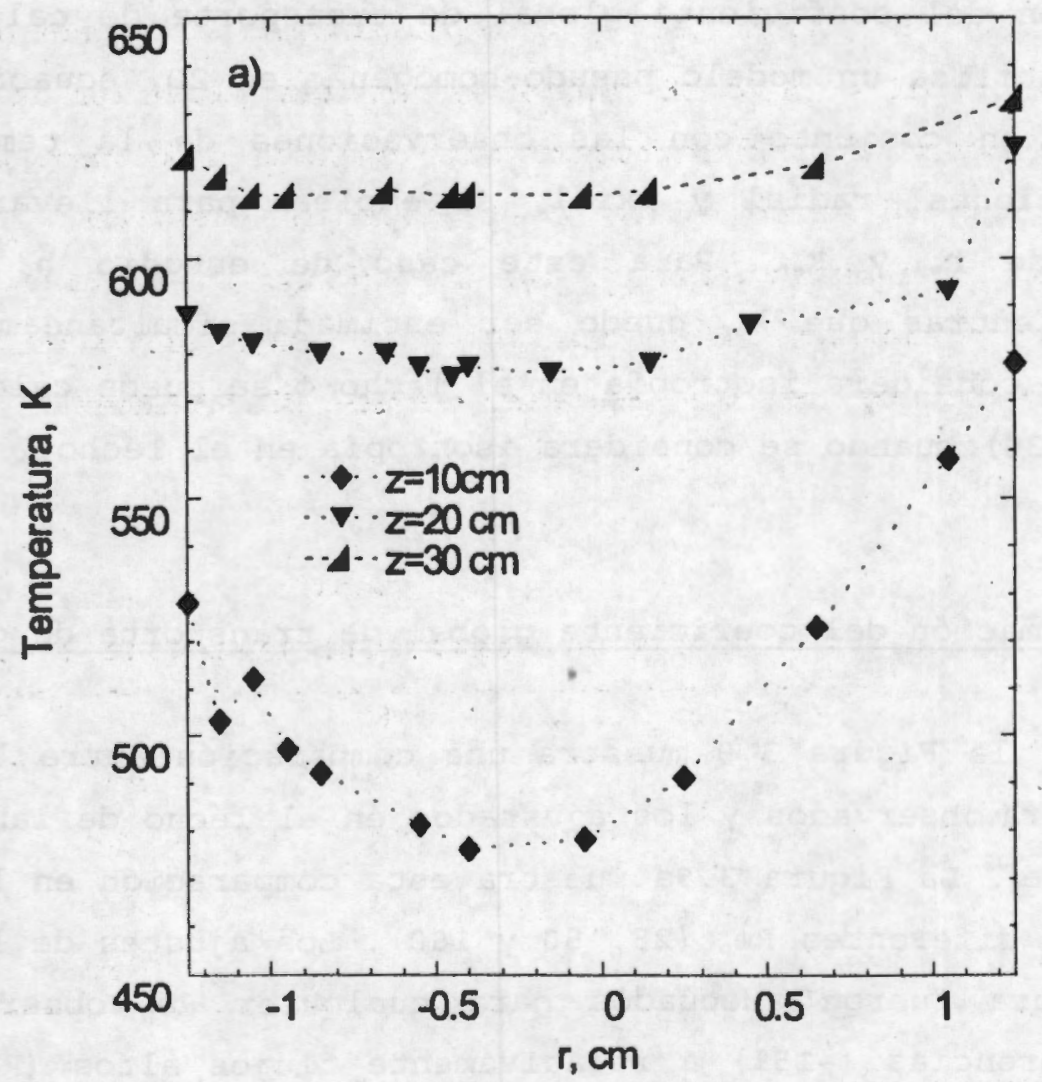

Figura 3.8. Perfiles de temperatura experimentales, en el lecho a escala piloto, en la dirección radial para un $R_{p}=630$ (Corrida 10 ).

En el ajuste de los perfiles de temperatura no se consideran las pérdidas de calor $y$ se utilizan los datos de temperatura del lado 
izquierdo de ambos tubos (ver Figura 3.3). No obstante, en trabajo a futuro estos perfiles de temperatura deben ser reconstruidos como lo muestra Lobez-Isunza et al. [26] .

\subsubsection{Estimación de los parámetros de transporte de calor: la aproximación clásica}

El ajuste de los perfiles de temperatura en el lecho empacado se llevó a cabo de dos formas. En la primera se utiliza un modelo pseudohomogéneo en 1D, ecuaciones $(3.9-3.12)$, que en conjunto con las observaciones de temperatura en la dirección axial, se emplearon para la estimación del coeficiente global de transporte de calor U. En la segunda se utiliza un modelo pseudo-homogéneo en 2D, ecuaciones (3.173.21c), que en conjunto con las observaciones de la temperatura en ambas direcciones, radial y axial, se emplean para llevar a cabo la estimación de $h_{w} y k_{a r}$. Para este caso de estudio $h_{w}$ siempre es estimado; mientras que $k_{e r}$ puede ser estimada simultáneamente con $h_{w}$ cuando no se considera isotropía en el lecho $\delta$ se puede calcular con la Ecuación (3.30) cuando se considera isotropia en el lecho.

\subsubsection{Estimación del coeficiente global de transporte de calor (U)}

Por un lado, la Figura 3.9 muestra una comparación entre los perfiles de temperatura observados y los ajustados en el lecho de laboratorio, a diferentes $R_{p}$. La Figura 3.9 a muestra esta comparación en la dirección axial a tres diferentes $\operatorname{Re}_{p}(25,50$ y 160). Los ajustes de los perfiles de temperatura fueron adecuadós para cualquier $R e_{p}$ observándose las mayores diferencias $(\sim 15 \%)$ a relativamente flujos altos $\left(R e_{p}=150\right)$. En la Figura $3.9 \mathrm{~b}$ se muestra esta comparación en la dirección radial (cuando se consideró un unico punto de colocación radial), en diferentes pociones axiales, a un $\mathrm{Re}_{\mathrm{p}}$ igual a 25 mostrando diferencias mayores al 30\%. 

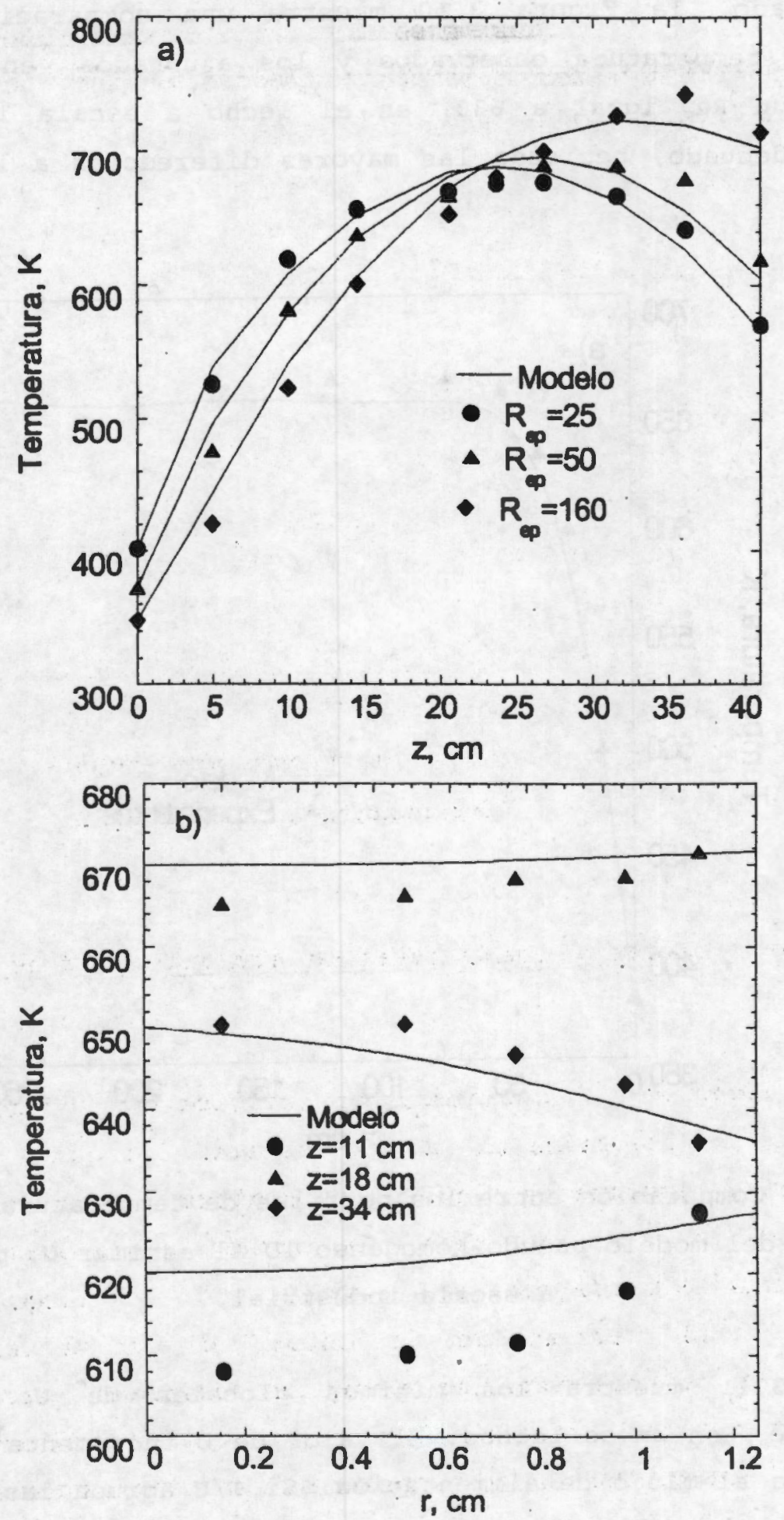

Figura 3.9. Comparación entre los resultados experimentales y los ajustes del modelo pseudo-homogéneo 1D. a) perfiles de temperatura axial a diferentes $\operatorname{Re}_{p} Y$ b) el perfil de temperatura radial a $R_{p}=25$. 
Por otro lado, la Figura 3.10 muestra una comparación entre los perfiles de temperatura observados y los ajustados, en la dirección axial y a un $\mathrm{Re}_{p}$ igual a 630 , en el lecho a escala industrial. El ajuste es adecuado, teniendo las mayores diferencias a la entrada del lecho.

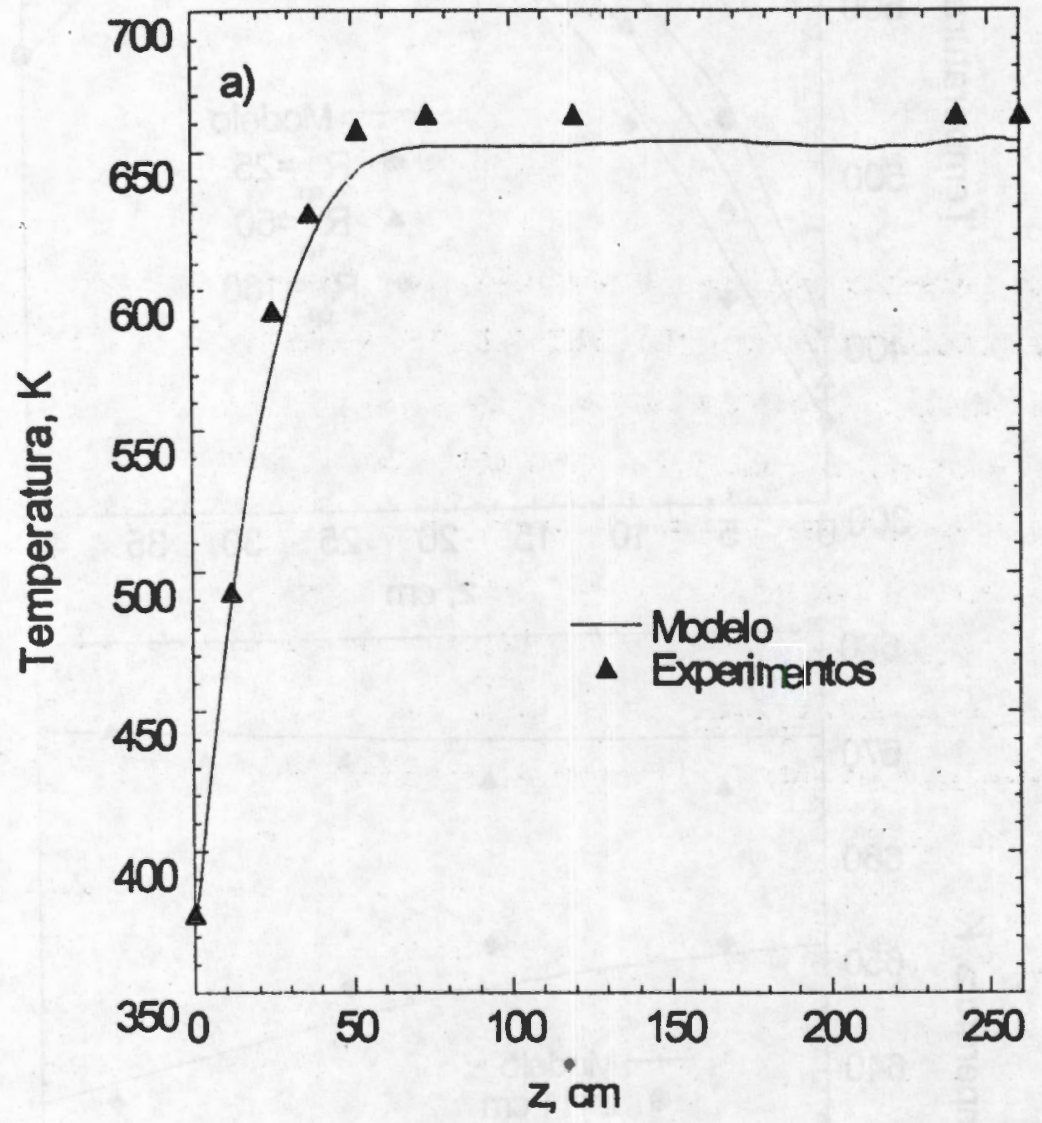

Figura 3.10. Comparación entre 10 perfiles de temperatura observados y los ajustes del modelo pseudo-homogéneo ID al estimar U, para el lecho a escala industrial.

La Figura 3.11 muestra los minimos globales de $U$ estimados, a diferentes $\mathrm{Re}_{\mathrm{p}}$, en ambos lechos. El valor de $\mathrm{U}$ incrementa a medida que se incrementa el flujo de alimentación. Si $1 / U$ agrupa las resistencias al transporte de calor total, la suma las resistencias en la región cercana a la pared $1 / h_{w} y$ en la parte central del lecho $1 / k_{\text {ar }}$, el incrementar el flujo de alimentación provoca que estas resistencias 
disminuyan, teniendo valores de $h_{w} y$ de $k_{a f}$ más altos (ver Tabla 3.3 ). Los valores estimados de $U$ se utilizan para modelar el reactor industrial estudiado. Esto es posible ya que ambos lechos manejan la misma relación de $d_{t} / d_{p} y$ utilizan el mismo empaque de catalizador que el industrial

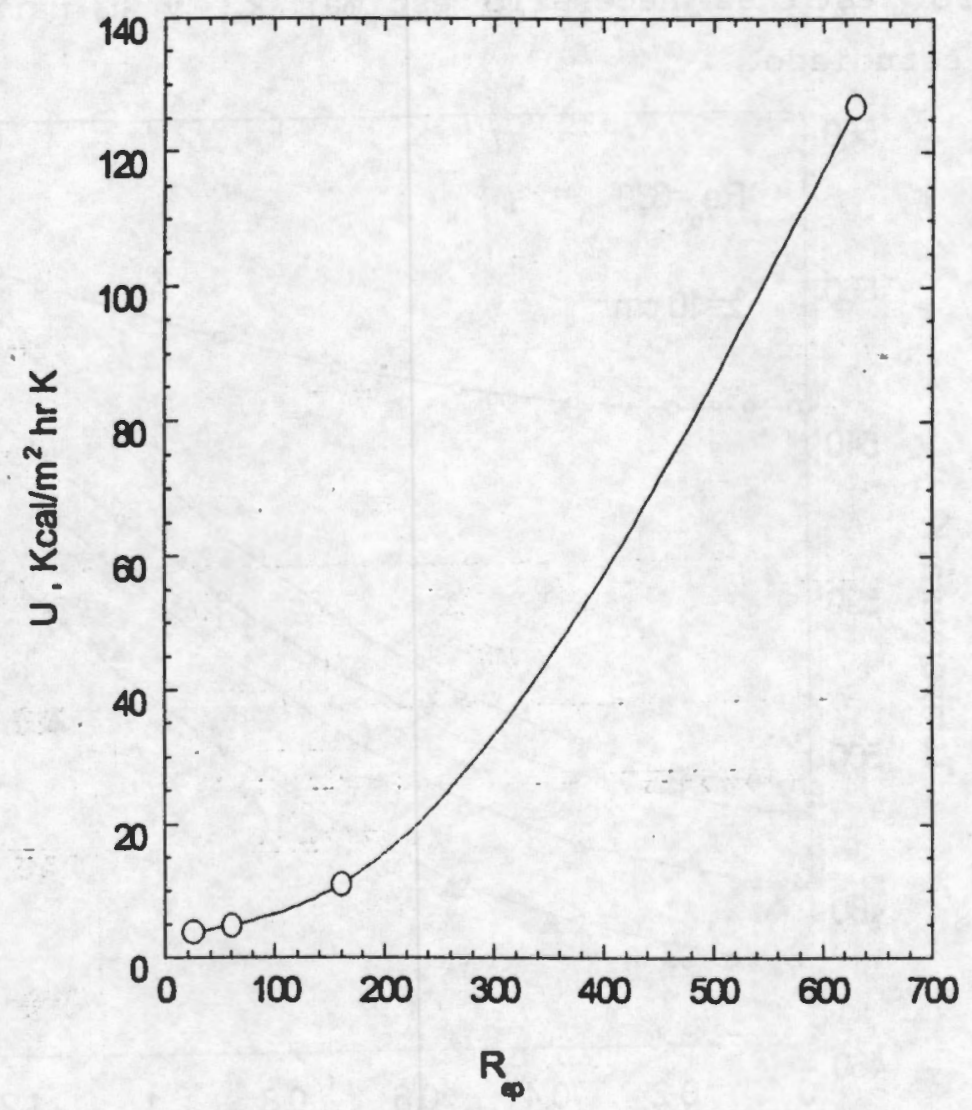

Figura 3.11. Estimación del coeficlente global de transporte calor en función del $\mathbf{R e}_{\mathrm{p}}$.

\subsubsection{Estimación del coeficiente de transporte de calor en la pared $\underline{h}_{w}$, y la conductividad térmica efectiva $k_{\text {er }}$}

La Figura 3.12 muestra la comparación de la predicción de los perfiles de temperatura que se obtienen en el lecho industrial a un $R e_{p}=630$,

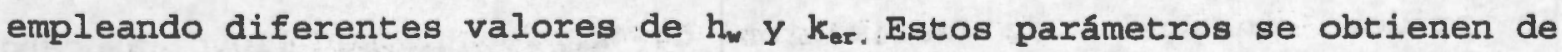
las correlaciones propuestas en la literatura $[14,115,116]$, de estimar 
ambos parámetros del modelo pseudo-homogéneo en $2 \mathrm{D}$ y de estimar estos parámetros con un modelo pseudo-heterogéneo (valores tomados de la literatura) [14]. Se observa que no existe una buena predicción de los perfiles de temperatura observados en el lecho industrial con los valores de $h_{w} y k_{e r}$ que se obtienen de correlaciones reportadas en la literatura. Por esto es necesario estimar $k_{\text {er }} y h_{w}$ para cada sistema experimental estudiado.

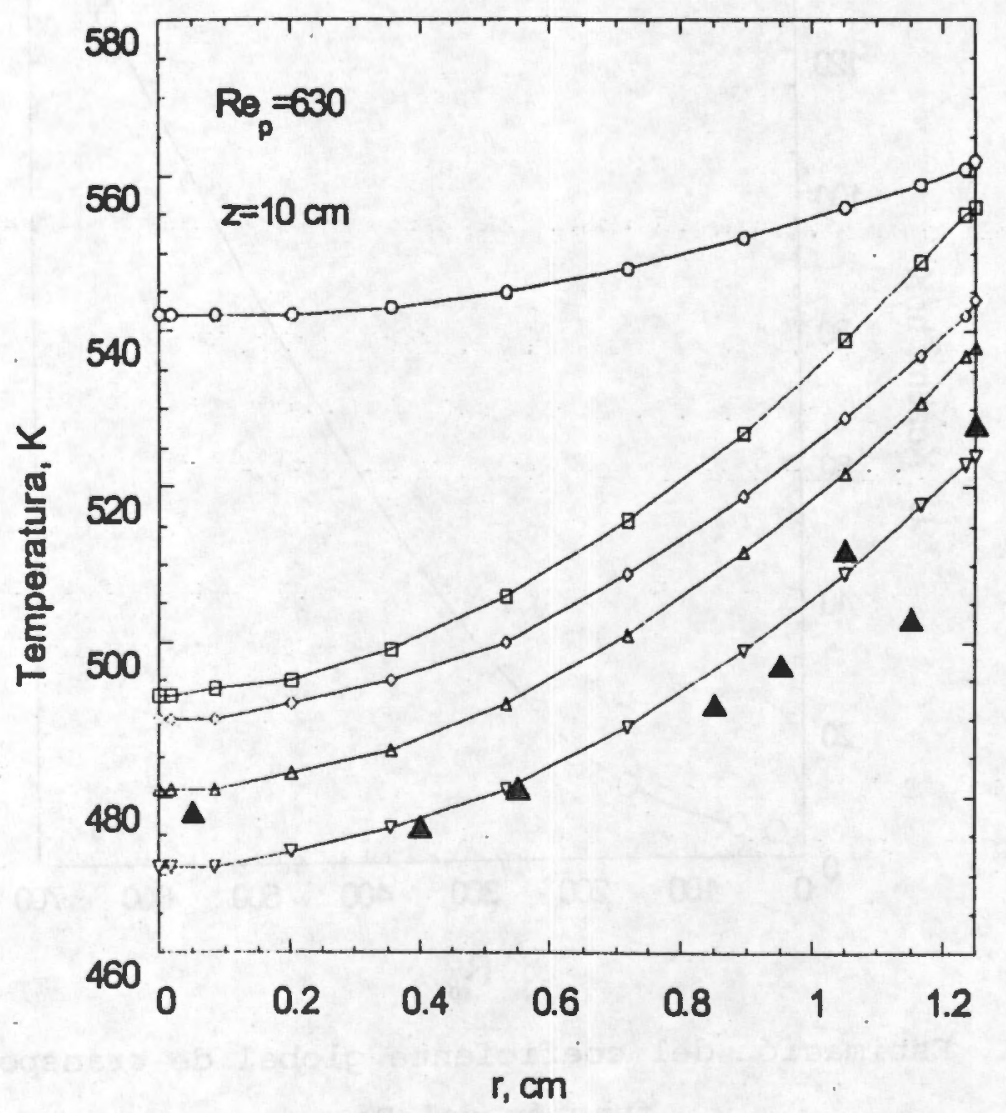

Figura 3.12. Comparación de los perfiles de temperatura experimentales Y los predichos al emplear distintas correlaciones para calcular los números de Peclet y Biot. (A) Experimentos, O) $P e=6, B_{1 w}=0.38[116]$, 口) $\mathrm{Pe}=18.62, \mathrm{~B}_{1 w}=1.05$-Demirel et al. [115] (0) Pe=16.4, $\mathrm{B}_{1 w}=0.8$ [15] ( $\left.\Delta\right)$ $\mathrm{Pe}=17.82, \mathrm{~B}_{\mathrm{iw}}=0.83$ [14] Y $(\nabla)$ estimados en este trabajo (Caso 1-B; Tabla 3.31

En primera instancia la estimación de $k_{e r} y h_{w}$ se llevó a cabo considerando dos casos: 
Caso 1-A) La aproximación clásica: el valor de $\boldsymbol{k}_{\text {ex }}$ se obtiene con la Ecuación (3.30) y hw es el único parámetro a estimar dentro del modelo pseudo-homogéneo en 2D.

Caso 1-B) La aproximación clásica: el valor de $k_{e r}$ que se obtiene con la Ecuación (3.30) se utiliza como valor inicial en la estimación de $k_{\text {er }}$ y $h_{w}$ dentro del modelo pseudo-homogéneo en $2 D$.

Las Figuras 3.13-3.20 muestran los ajustes de los perfiles de temperatura observados para los casos $1-A$ y $1-B$ en el rango de $R e_{p} 25-$ 630 en ambos lechos (de laboratorio e industrial). Estos casos se compararon con aquéllos que incluyen el modelo hidrodinámico en la estimación de $k_{\text {er }} y h_{w}, y$ que se discuten en la siguiente sección. Los perfiles de temperatura ajustados en la dirección axial son adecuados para los casos 1-A y 1-B siendo menos satisfactorios conforme el $\mathrm{Re}_{\mathrm{p}}$ incremento. Haciendo dificil decir cual aproximación es la mejor. El ajuste adecuado de las observaciones en ambos lechos, utilizando el caso 1-B, muestra que la hipotesis planteada de utilizar dos distintos sistemas experimentales, adiabático y no-adiabático, para estimar un parámetro de transporte de calor en cada experimento bajo la consideración de isotropia permitio eliminar las incertidumbres de tener que estimar dos parámetros de transporte de calor utilizando un solo tipo de experimento (Caso 1-A). Aunque, el ajuste fue adecuado para ambos casos, los valores estimados de $h_{w} y \quad k_{a r}$ mostraron diferencias alrededor del 1-20\%; esta diferencia se incrementó conforme el flujo de alimentación también incremento (ver la Tabla 3.3).

\subsubsection{Estimación de los parámetros de transporte de calor: incorporación de los perfiles de fracción vacia y velocidad}

Como se comentó anteriormente, los perfiles de fracción vacía y velocidad juegan un papel importante en la prediccion de los perfiles de temperatura en el lecho estudiado (ver Apéndice G) [14]. Por lo 
consiguiente, los siguientes cuatro casos consideran la hidrodinámica, más el perfil de fracción vacía, para estimar $h_{w} y k_{\text {er }}$ de experimentos en el lecho que opera de forma no-adiabática (tanto de laboratorio como escala industrial), para un rango de $R_{p}$ entre 25 y 630 :

Caso 2-A) La hidrodinámica: el valor de $k_{\text {er }}$ se calcula con la Ecuación (3.30); este valor se emplea para predecir los perfiles de temperatura observados. Se utiliza el modelo pseudo-homogéneo $2 D$ y la condición de frontera (21.b).

Caso 2-B) La hidrodinámica: $k_{\text {er }}$ es el único parámetro a estimar en el modelo pseudo-homogéneo $2 \mathrm{D}$, utilizando la condición de frontera (21.b).

Caso 3-A) La aproximación de la capa limite: $k_{e r}$ se calcula con la Ecuación (3.30), y $h_{w}$ es el único parámetro a estimar en el modelo pseudo-homogéneo 2D, utilizando la condición de frontera (21.a).

Caso 3-B) La aproximación de la capa limite: los parámetros $h_{w} y k_{a x}$ se estiman simultáneamente empleando la condición de frontera (21a) y el modelo pseudo-homogéneo 2D; la ecuación (3.30) proporciona los valores iniciales de la estimación $k_{a r}$.

En las Figuras 3.13 a 3.20 se muestra una comparación entre los ajustes de los perfiles de temperatura, que se obtuvieron bajo las consideraciones de los casos 1-3 (A Y B), Y las observaciones de temperatura en el lecho. Las Figuras 3.13 a 3.18 muestran los perfiles de temperatura que se tienen en el lecho de laboratorio ( $\left.R e_{p}=25-150\right)$; mientras que en la Figuras 3.19 y 3.20 se presentan los perfiles de temperatura que se tienen en el lecho a escala industrial $\left(R_{p}=630\right)$.

El análisis de los perfiles de temperatura ajustados, a un $\mathrm{Re}_{\mathrm{p}}$ bajo $(<160)$, da lugar a la siguiente discusión:

i) Los perfiles de temperatura radiales ajustados, a un $\operatorname{Re}_{p}$ igual a 25. con los casos 2 y 3 (A Y B) son menores al $5 \%$ con respecto a 
los experimentales y los ajustados con la aproximación clásica (Casos 1 (A Y B)). Observando como principal diferencia que los perfiles de temperatura ajustados con la aproximación clásica son parabblicos. Para todos los casos, el ajuste de los perfiles de temperatura para $\mathrm{Re}_{\mathrm{p}}$ mayores son adecuadas, aún cuando hay problemas en el ajuste a la entrada del lecho $y$ al incrementar el flujo de alimentación.

ii) El ajuste es razonablemente bueno para los perfiles de temperatura observados en la dirección axial para todos los casos, aún cuando el ajuste se complica cuando se incrementa el flujo.

iii)Los resultados muestran un ajuste ligeramente mejor, de los perfiles de temperatura (en las direcciones axial y radial), cuando el valor de $k_{a x}$ se estima a partir de experimentos en el lecho noadiabático (Casos B). Sin embargo, el valor de $k_{\text {ex }}$ estimado muestra diferencias hasta de un 10 fon respecto a aquel obtenido en los casos A (ver Tabla 3.3).

El análisis de los perfiles de temperatura ajustados, cuando se manejo un $R e_{p}$ igual a 630 , da lugar a la siguiente discusión:

i) La comparación de los perfiles de temperatura ajustados, en la dirección radial, para los tres casos (A y B), no fue fácil por la distorsión de los perfiles de temperatura que se tienen en el lecho. Sin embargo, el ajuste de estos perfiles, en tres posiciones axiales, es tan buena para la aproximación clásica como para aquella en donde se incorpora la hidrodinámica; lo cual resulta contradictorio a comentarios expuestos en recientes investigaciones [115].

ii) Los perfiles de temperatura ajustados, fueron ligeramente mejor, para los casos 1B, 2A Y 3A, existiendo diferencias menores al 108 entre estas. No obstante, el caso $2 \mathrm{~A}$ esta libre de la incertidumbre que genera la estimación de $h_{w}$ ya que solamente se estima un solo 
parámetro; por esto, se sugiere que el parámetro estimado bajo las consideraciones de este caso (siempre y cuando se conozca la temperatura interna de la pared), se debe utilizar en la simulación del reactor.

Una de las limitaciones para ajustar los perfiles de temperatura, cuando se introdujo la hidrodinámica en el lecho empacado, es que la velocidad radial es despreciable y la posible presencia de fenómenos de turbulencia no se consideraron en el modelo. Por lo que, futuros estudios van dirigidos a buscar una forma de considerar los campos de velocidad (para ambas componentes: axial y radial) y emplear modelos turbulentos que describan más rigurosamente la hidrodinámica en el lecho empacado.

La Tabla 3.3 da los valores estimados de $k_{\text {ex, }} h_{w}$, $P e y$ $B_{1 w}$ para cualquiera de los tres casos (A Y B). El Pe incrementó conforme lo hizo

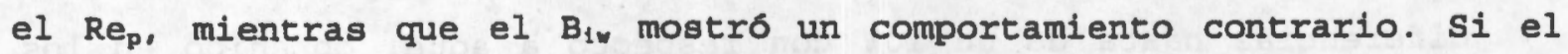
$B i_{w}$ disminuye al incrementar el $R_{p}$, el valor de $k_{e r}$ se incrementa más rápidamente que $h_{w}$, es decir, la resistencia al transporte de calor en el centro del lecho $\left(1 / k_{e r}\right)$ disminuye más rápido que en la pelicula del fluido inmediata a la pared del lecho $\left(1 / h_{w}\right)$; esto provoca que los perfiles de temperatura radiales, sean más pronunciados cerca de la pared que en la parte central del lecho.

Como se comentó anteriormente, $k_{\text {er }} y h_{w}$ se calculan como la suma de dos contribuciones (ver la Ecuación (3.30)): una estática y una dinámica. La contribución estática decrece conforme se incrementa la fracción vacía del lecho empacado (que es cuando se tiene una relación $d_{\varepsilon} / d_{p}$ (10). La contribución dinámica es función de los números de Reynolds $\left(R e_{p}\right), y$ de prandtl $\left(P_{r}\right)$; si se trabaja en un lecho con una relación de $d_{c} / d_{p}<10$, podría también ser función de la velocidad intersticial. En este estudio, para los casos 2 y 3 (A $y$ B), $k_{\text {er }}$ y $h_{w}$ se estimaron agrupando todos los efectos hidrodinámicos en los procesos de transporte de calor en la dirección radial y axial; es decir, estos parámetros no fueron una función de la posición radial y axial lo mejor 
dicho de la velocidad intersticial), y su estimación se realizó siguiendo la forma clásica. En trabajos futuros, se sugiere que los parámetros de transporte de calor deben evaluarse como una función de la contribución estática y dinámica, lo que permitiría observar el efecto de la velocidad intersticlal en las resistencias al transporte de calor en este tipo de sistemas.

Tabla 3.3.

Valores estimados de los parámetros de transporte de calor. Pe $y k_{0 r}$

\begin{tabular}{|c|c|c|c|c|}
\hline$R_{a p}$ & Casos A & Caso IB & Саво 2B & Caso $3 B$ \\
\hline 25 & 4.3 & 4.3 & 4.9 & 3.9 \\
\hline 60 & 5.1 & 7.1 & 7.9 & 6.9 \\
\hline 160 & 7.5 & 7.5 & 9.1 & 10.0 \\
\hline 630 & 15.5 & 16.5 & 14.1 & 14.1 \\
\hline \multicolumn{5}{|c|}{$k_{\mathrm{ax}}, \mathrm{J} \cdot \mathrm{m}^{-1} \cdot \mathrm{s}^{-1} \cdot \mathrm{x}^{-1}$} \\
\hline 5 & 0.36 & 0.36 & 0.31 & 0.39 \\
\hline 60 & 0.60 & 0.43 & 0.39 & 0.44 \\
\hline 160 & 1.31 & 1.31 & 1.08 & 0.98 \\
\hline 630 & 2.47 & 2.32 & 2.71 & 2.70 \\
\hline \multicolumn{5}{|c|}{$\mathrm{Bi}_{\text {r }} \mathrm{Y} \mathrm{h}$} \\
\hline & Caso 1A & Caso 1B & Caso 3A & Caso 3B \\
\hline 25 & 9.1 & 9.1 & 9.8 & 7.4 \\
\hline 60 & 7.8 & 8.5 & 7.5 & 6.5 \\
\hline 160 & 5.4 & 4.3 & 4.4 & 3.1 \\
\hline 630 & 0.8 & 0.7 & 1.4 & 1.3 \\
\hline \multicolumn{5}{|c|}{$h_{m,}, 3 \cdot m^{2} \cdot s^{-1} \cdot K^{-1}$} \\
\hline 25 & 261.9 & 261.3 & 282.4 & 227.3 \\
\hline $60^{\circ}$ & 374.5 & 294.3 & 361.0 & 230.2 \\
\hline 160 & 564.9 & 451.0 & 461.3 & 242.8 \\
\hline 630 & 158.2 & 130.0 & 286.7 & 292.4 \\
\hline
\end{tabular}



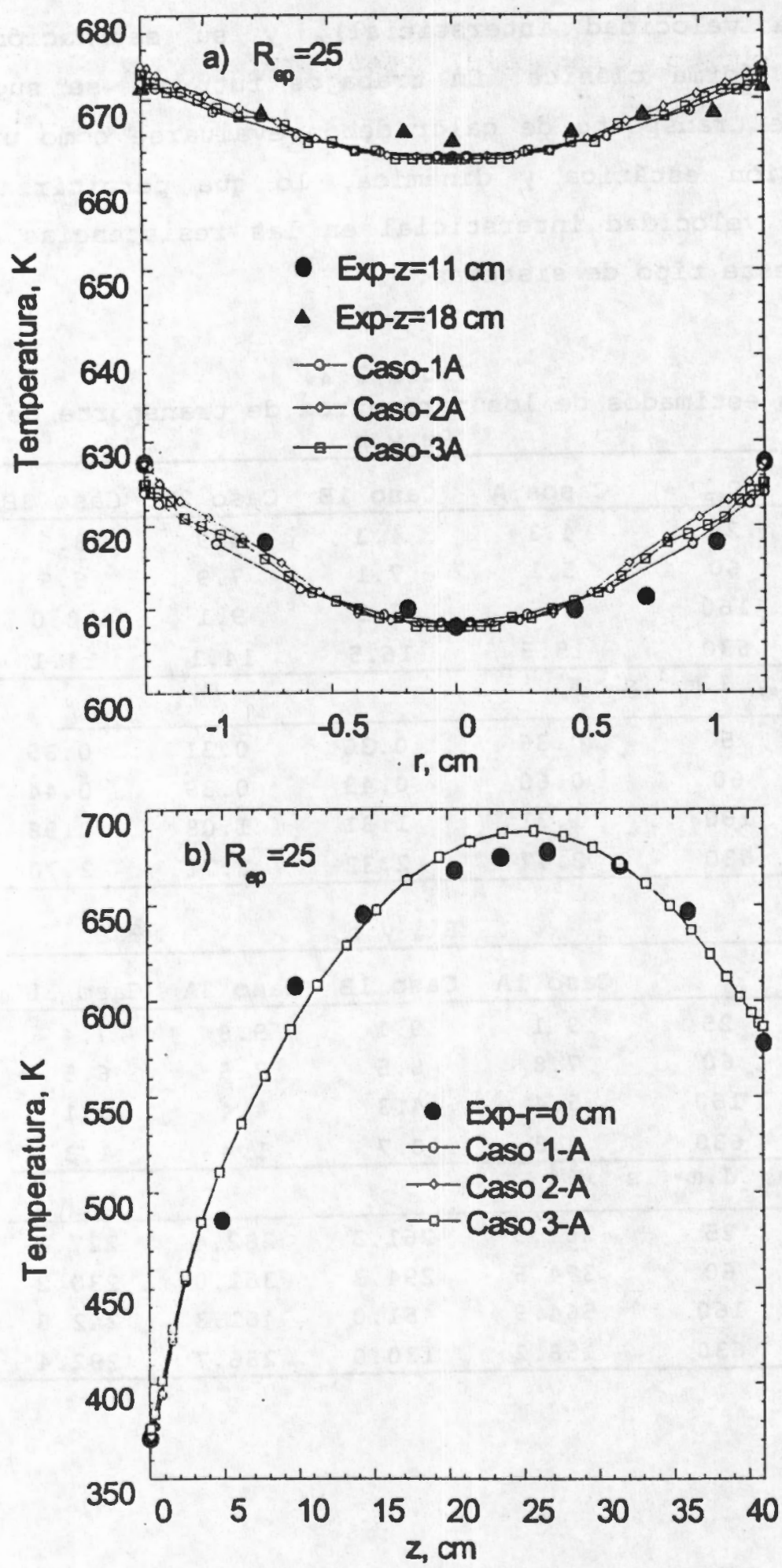

Figura 3.13. Ajuste de los perfiles de temperatura, estimando unicamente $h_{w}$ para un $R_{p}=25$. En las direcciones: a) radial $y$ b) axial. 

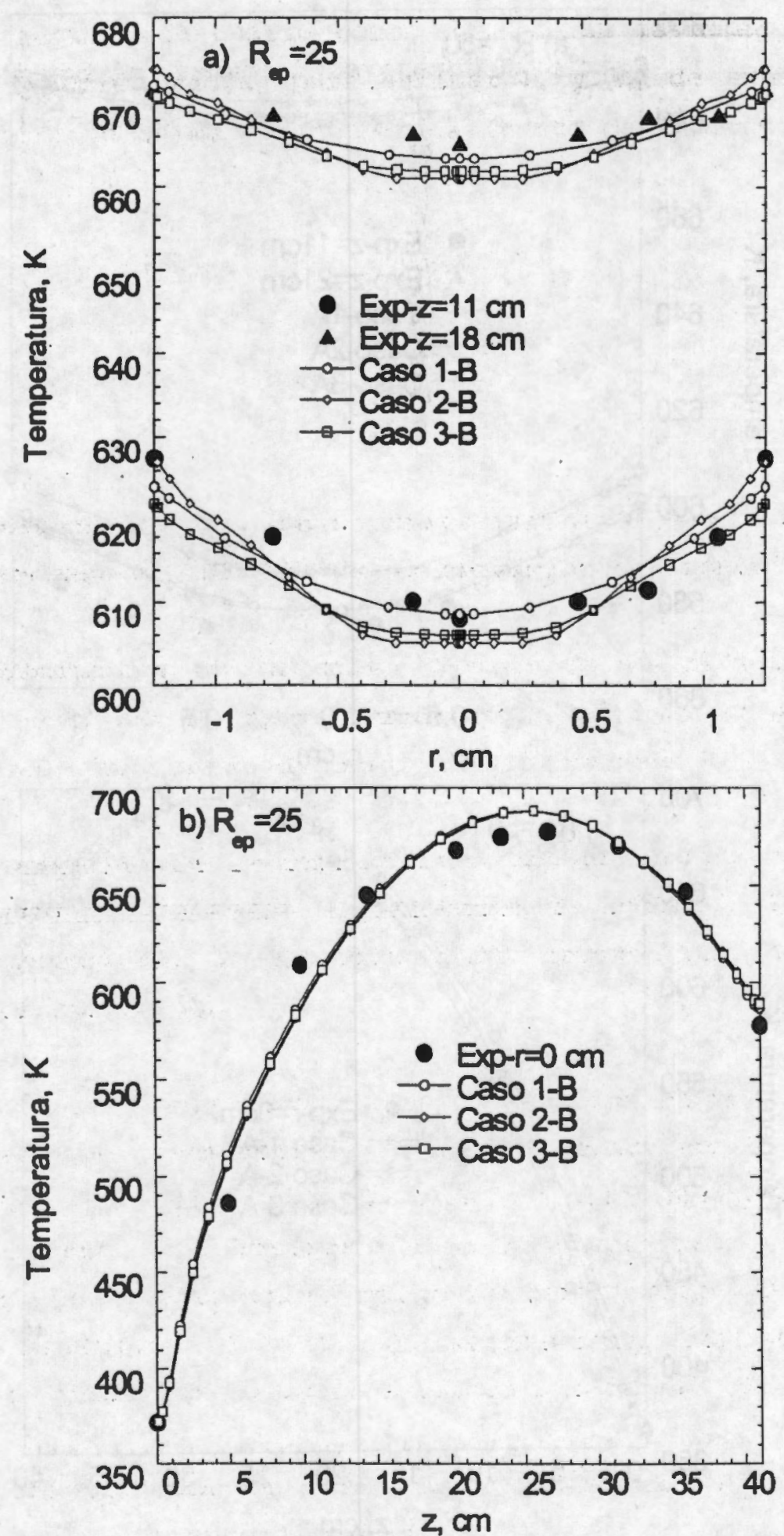

Figura 3.14. Ajuste de los perfiles de temperatura estimando $h_{w} y k_{m}$ para un $R e_{p}=25$. En las direcciones: a) radial y b) axial. 

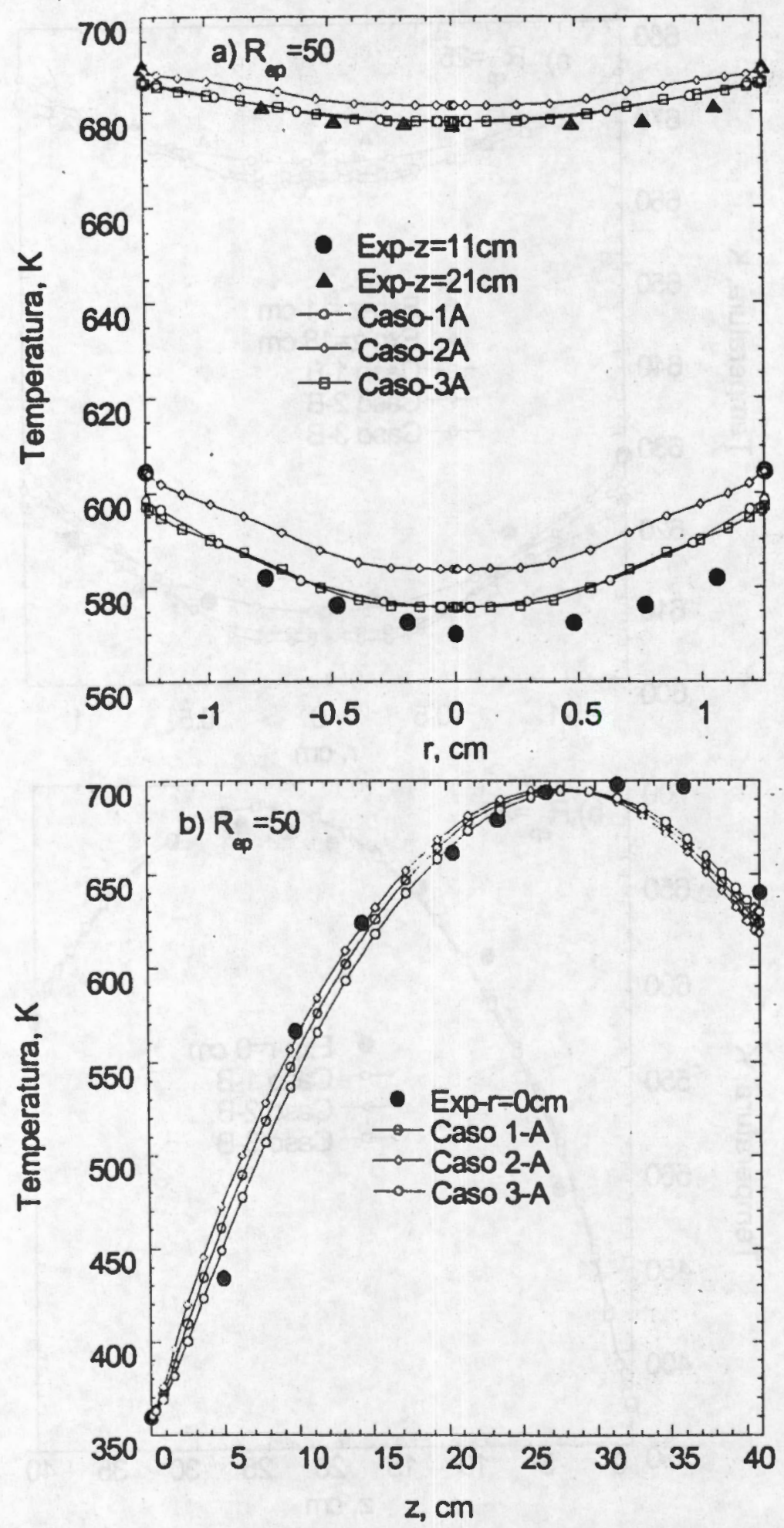

Figura 3.15. Ajuste de los perfiles de temperatura estimando funicamente $h_{w}$ para un $R e_{p}=50$. En las direcciones: a) radial y b) axial. 

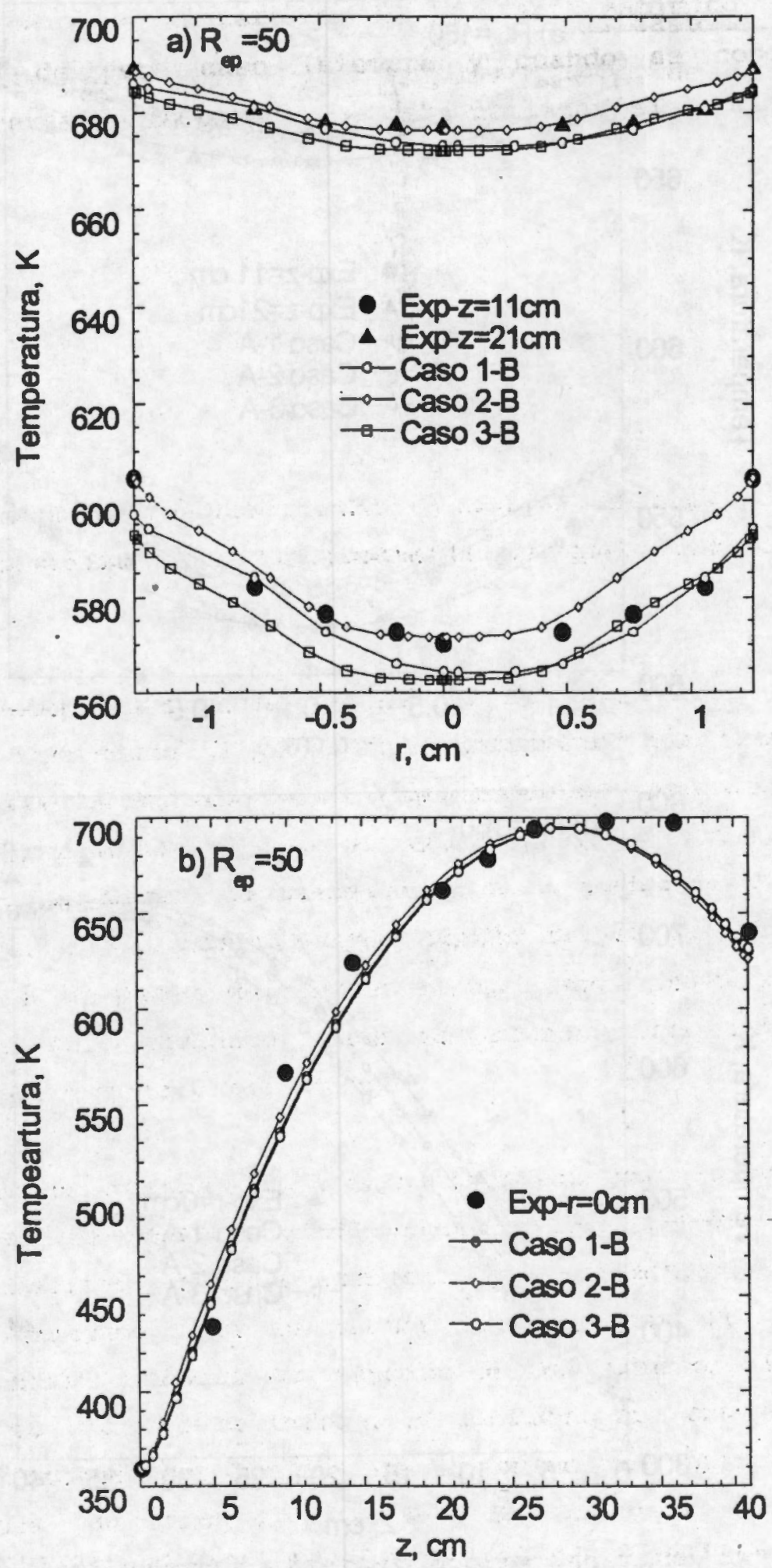

Figura 3.16. Ajuste de los perfiles de temperatura estimando $h_{w} y k_{e r}$ para un $R_{p}=50$. En las direcciones: a) radial y b) axial. 

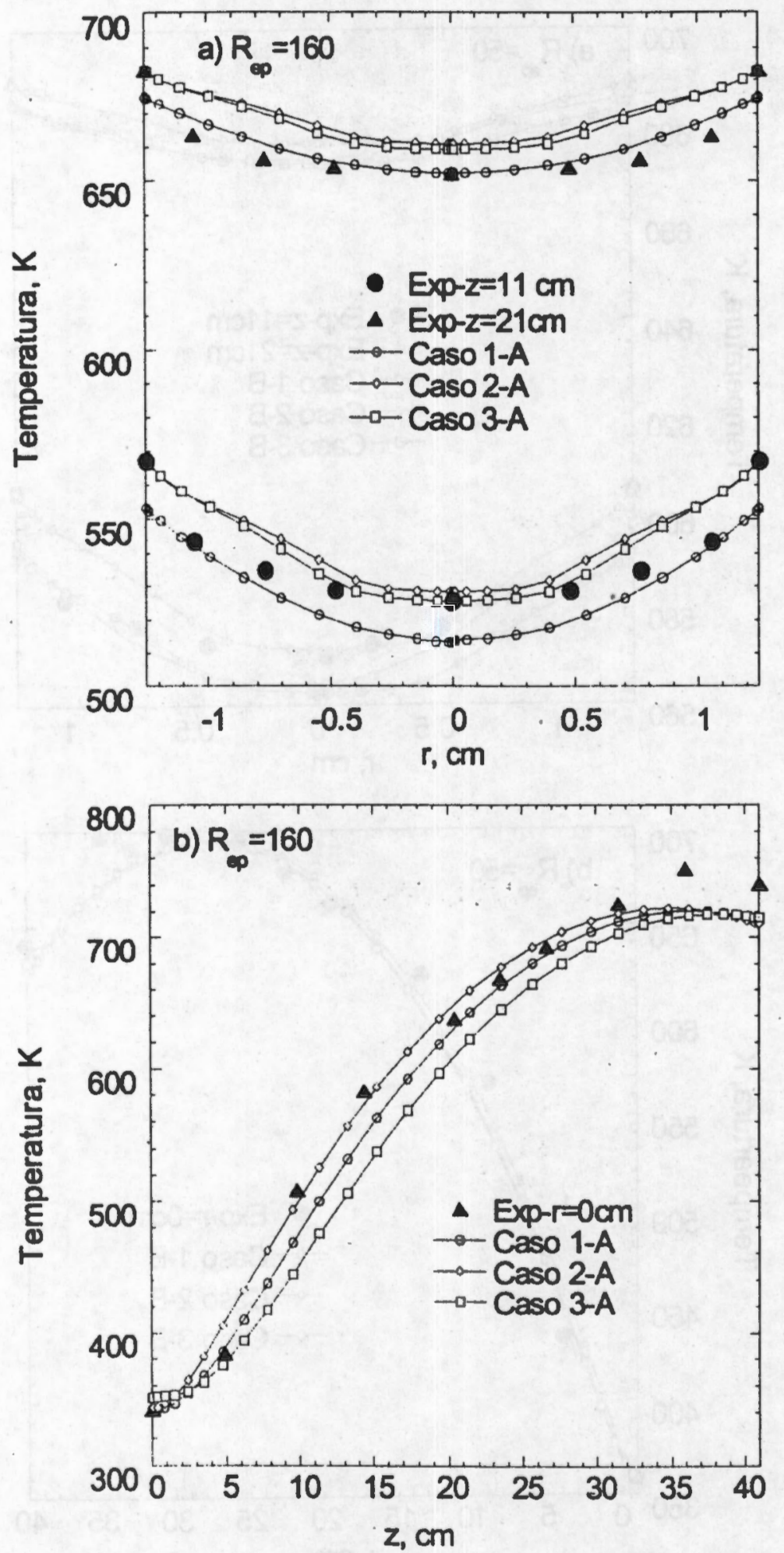

Figura 3.17. Ajuste de los perfiles de temperatura estimando únicamente $h_{w}$ para un $R_{c p}=160$. En las direcciones: a) radial $y$ b) axial. 

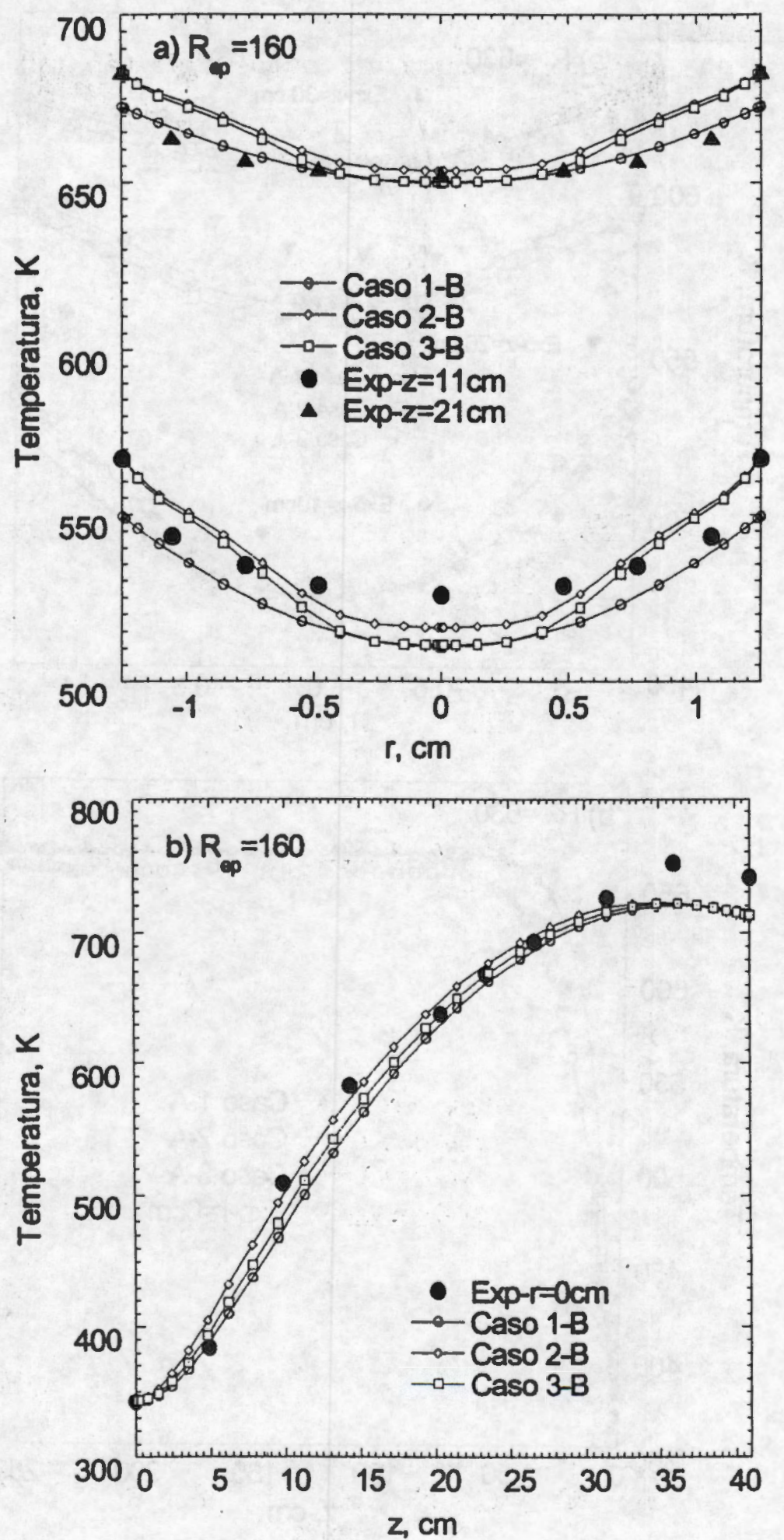

Figura 3.18. Ajuste de los perfiles de temperatura estimando $h_{w} y k_{a r}$ para un $R e_{p}=160$. En las direcciones: a) radial y b) axial. 

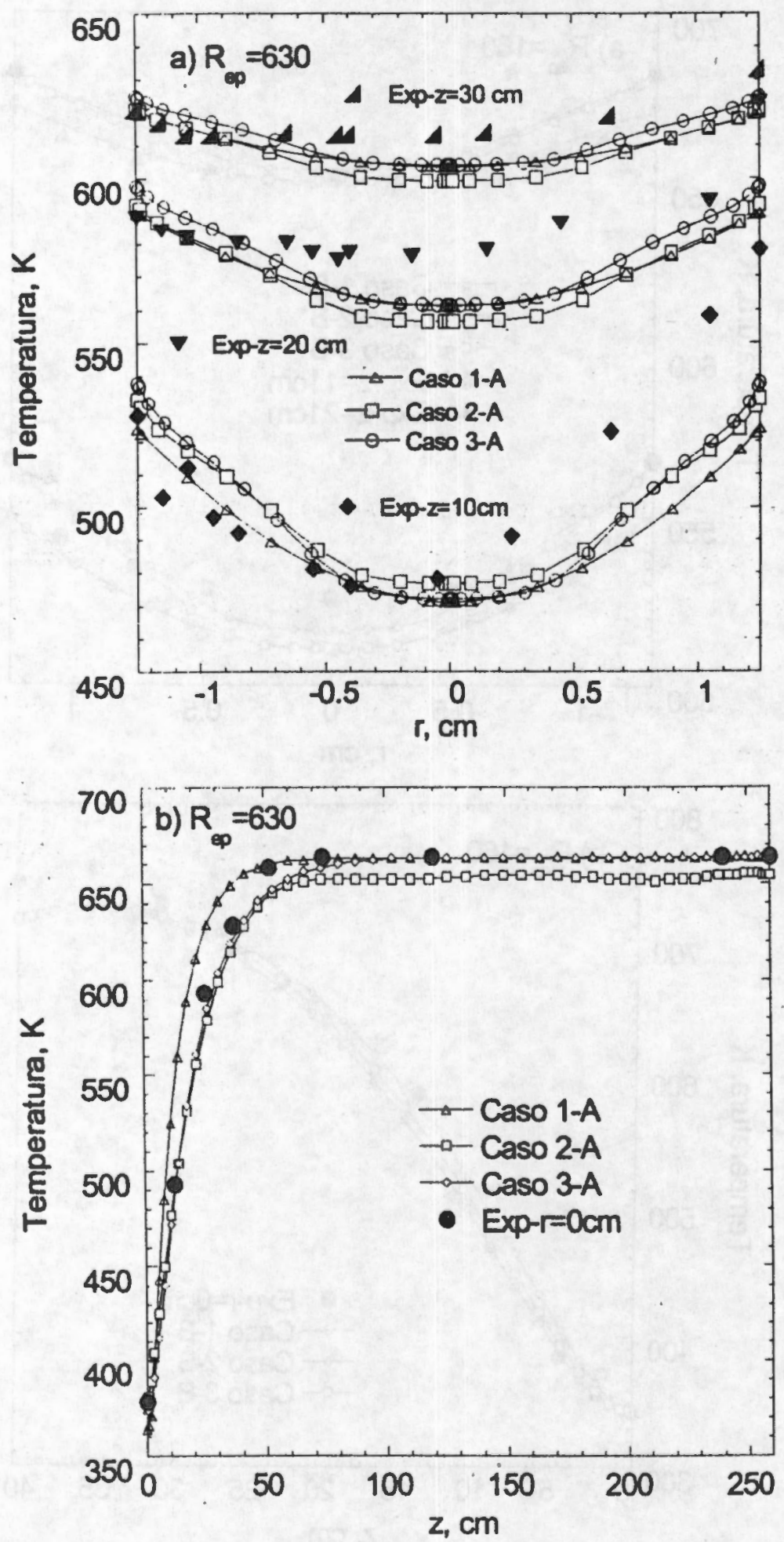

Figura 3.19. Ajuste de los perfiles de temperatura estimando únicamente

$h_{w}$ para un $R e_{p}=630$. En las direcciones: a) radial y b) axial. 

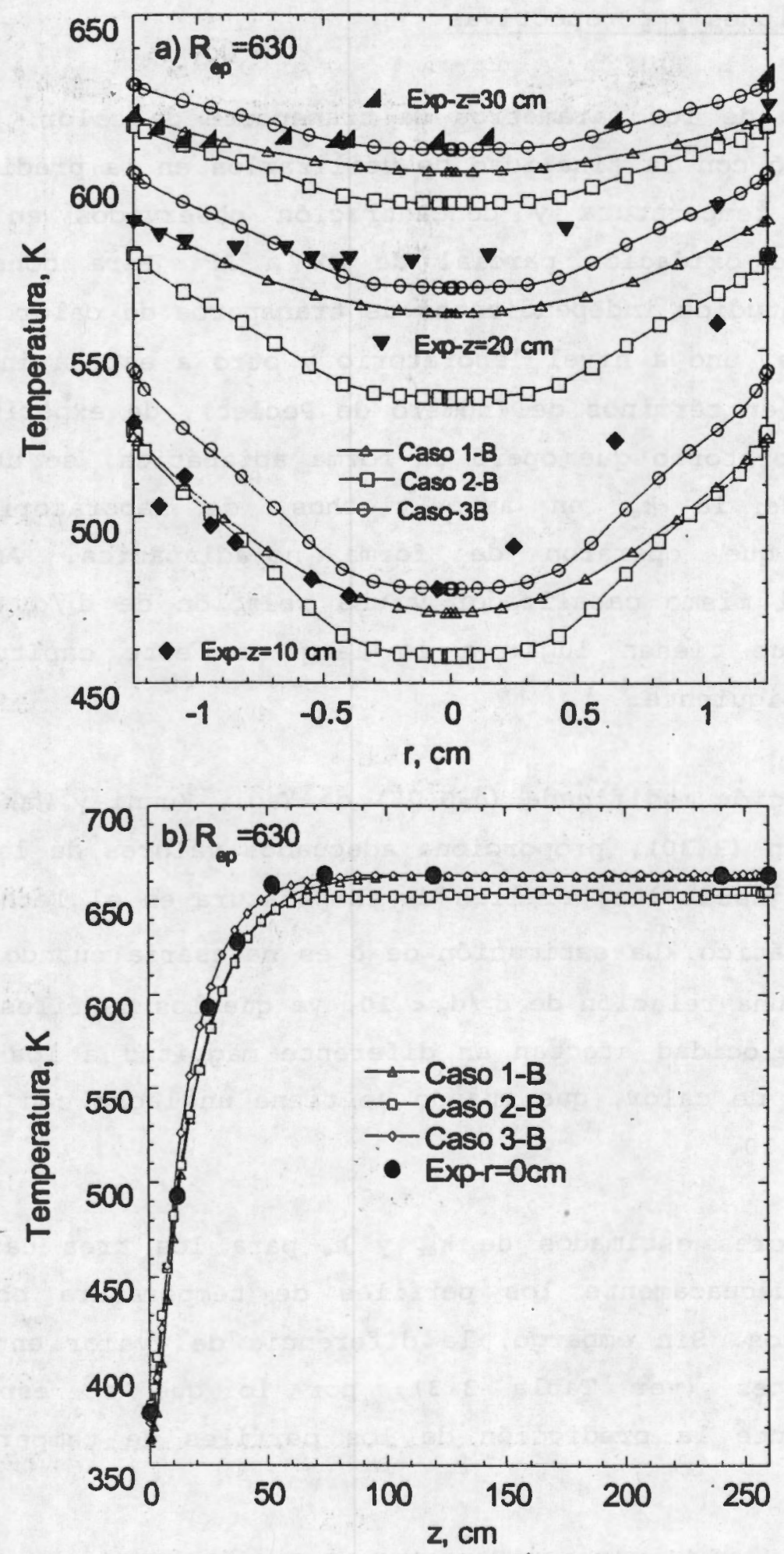

Figura 3.20. Ajuste de los perfiles de temperatura estimando $h_{w} y k_{a r}$ para un $R e_{p}=160$. En las direcciones: a) radial $y$ b) axial. 


\subsection{Conclusiones y perspectivas}

La estimación de los parámetros de transporte de calor, $U, k_{e z}, k_{e r} y$ $h_{w}$, se realizb con la finalidad de utilizarlos en la predicción de los perfiles de temperatura $Y$ concentración observados en el reactor industrial de oxidación parcial de OX a AF. Para conseguirlo, se realizaron estudios independientes de transporte de calor sin reacción en dos lechos, uno a nivel laboratorio y otro a escala industrial. La $\mathrm{k}_{\mathrm{ez}}$ estimada (en términos del número de Peclet), de experimentos en un lecho de laboratorio que operó en forma adiabática, se utilizó en la estimación de la $k_{e r}$ en ambos lechos, de laboratorio $y$ escala industrial, que operaron de forma no-adiabática. Ambos lechos utilizaron el mismo catalizador y una relación de $d_{c} / d_{p}$ similar. Los resultados que tienen lugar a lo largo de este capitulo permiten concluir lo siguiente:

1) La correlación modificada $(\delta=0.75)$ de Yagi, Kunni y Wakao, dada por la Ecuación (3.30), proporciona adecuados valores de la $k_{e z}$, ya que permitio ajustar los perfiles de temperatura en el lecho adiabático y no-adiabático. La estimación de $\delta$ es necesaria cuando se tiene un lecho con una relación de $d_{c} / d_{p}<10$, ya que los perfiles de fracción vacia y velocidad afectan en diferente magnitud a los procesos de transporte de calor, que cuando se tiene un lecho con una relación de $d_{t} / d_{p}>10$.

i1) Los valores estimados de $k_{\text {er }} y h_{w}$ para los tres casos (A $y$ B) ajustan adecuadamente los perfiles de temperatura observados en ambos lechos. Sin embargo, la diferencia del valor entre estos es significantes (ver Tabla 3.3), por lo que se espera afecten sensiblemente la predicción de los perfiles de temperatura en el reactor.

iii) El ajuste de los perfiles de temperatura al utilizar la aproximación clásica (Casos 1-A y 1-B) es tan buena como al 


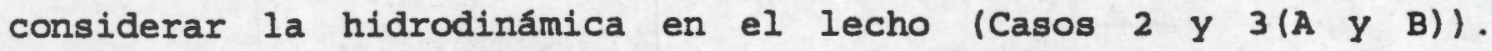
Resultados que son contradictorios a lo reportado en la literatura.

iv) Los casos 2, que acoplan el modelo hidrodinámico al de transporte de calor, requieren lecturas de temperatura en el interior de la pared; si no se cuentan con ellas, los casos tres, que utilizan la aproximacion de capa límite, puede utilizarse para la estimación de $k_{\text {ex }} \mathrm{Y} \mathrm{h}_{\mathrm{w}}$.

Finalmente, se sugiere abordar lo siguiente para futuros trabajos. 1) Considerar un modelo hidrodinámico riguroso, que considere los perfiles de fracción en las tres dimensiones, la componente de la velocidad radial, y la turbulencia presente en el lecho. Lo cual parece ser esencial $y$ tener un efecto importante en la estimación de los parámetros de transporte de calor. 2) Los parámetros de transporte de calor, $k_{e r}, y h_{w}$ deben considerar sus contribuciones, estática $y$ dinámica, para observar el efecto de los campos de velocidad en las resistencias al transporte de calor en cada zona del reactor. 

reactor de oxidación parcial de OX a AF

\section{Capítulo 4.}

Estudio del comportamiento en estado

estacionario del reactor de oxidación parcial de o-xileno a anhídrido ftálico 

reactor de oxidación parcial de OX a AF

4. Estudio del comportamiento en estado estaclonarlo del reactor de oxidactón parciel de o-xileno a anhidrido ftilleo

En este capitulo se discute el comportamiento del reactor de oxidación parcial de o-xileno (OX) a anhidrido ftálico (AF) en el estado estacionario. Para ello éste se divide en tres partes:

En la primera parte se revisa brevemente el estado del arte sobre la reacción estudiada. La sección 4.1 da algunos antecedentes sobre el modelamiento de este reactor en estado estacionario. En la sección 4.2 se revisa brevemente el comportamiento catalitico del catalizador de $\mathrm{V}_{2} \mathrm{O}_{3} / \mathrm{TiO}_{2}$ en las reacciones de oxidación parcial de hidrocarburos $y$ en la sección 4.3 se muestran los modelos cinéticos reportados en la literatura destacando sus principales cualidades e inconsistencias.

En la segunda parte, la sección 4.4 muestra las principales caracteristicas del sistema experimental. En la sección 4.5 se presentan los resultados experimentales que se tienen en el reactor, mientras que en la sección 4.6 se dan 108 modelos del reactor que describen su comportamiento fenomenológico.

Finalmente, con el objetivo de estudiar las principales fuentes de incertidumbre en el modelamiento del reactor de oxidación parcial de Ox, la tercera parte se divide en dos secciones. En la sección 4.7 se estudia el impacto de diferentes factores, es decir la desactivación del catalizador, los procesos de transporte de momento, de calor y de masa, en la capacidad de predicción del modelo del reactor. En la sección 4.8 se predice el comportamiento observado en el reactor al utilizar los parámetros de transporte de calor estimados bajo la consideración de isotropía y no isotropia utilizando la aproximación clásica y aquélla que considera a los perfiles de velocidad. En la sección 4.9 se presentan las conclusiones y perspectivas. 

reactor de oxidación parcial de OX a AF

4.1 Antecedentes sobre el estudio del reactor en el estado estacionario

La mayoría de los autores $[8,12,13-16,18,22,23,25,29-34,87,125,135,137$ 140,144,145] han enfocado sus investigaciones a tratar de predecir $y$ explicar el comportamiento observado del reactor. Algunos de éstos han tenido como objetivo prevenir un comportamiento fuera de control del reactor (runaway) y de esta forma proponer diferentes criterios que den como resultado una operación segura del reactor, por ejemplo menores temperaturas en la posición del punto caliente, mayores conversiones de OX Y altas selectividades a AF. Sin embargo, diversas publicaciones teórico-experimentales [14-16,19] han demostrado que estudios teoricos no predicen el comportamiento observado en el reactor industrial $[87,101]$.

Los estudios cinéticos que aparentan ser los más exitosos en la actualidad, soiore la oxidación parcial de o-xileno son los realizados por Calderbank et al. (1977) [8], Skrzypek et al. (1985) [17] y Papageorgiou et al. (1994) [132]. Ellos proponen y evalúan modelos cinéticos utilizando un catalizador industrial $y$ en condiciones de operación cercanas a las industriales. Estos estudios utilizan un reactor de lecho fijo con una relación de $d_{\mathfrak{c}} / d_{p} \sim 6$. Para evitar gradientes de temperatura radiales importantes, el catalizador se diluye con partículas inertes de la misma forma y tamaño. El modelo del reactor considera las dos fases (sólido-gas), en tanto que las dispersiones axial y radial, de masa y de calor, se desprecian. Estos modelos predicen los resultados experimentales de sus sistemas de reacción, sin embargo, los parámetrog cinéticos no se evallian para un catalizador con desactivación. En la actualidad, para tener una predicción adecuada de los perfiles de temperatura y concentración en esta clase de reactores, los parámetros cinéticos son re-estimados $[23,34]$ o se incorporan expresiones empiricas en el término de reacción (en el modelo del reactor), con el propósito de considerar la desactivación que sufre el catalizador [28-33]. Actualmente el modelo 
más usado e para predecir el comportamiento cinético del catalizador es el propuesto por Calderbank et al. $[8,12,13-16,18,19]$.

En diversas publicaciones $[13-16,30,41,43,61,88,89,135-139]$ se ha estudiado el efecto de los fenómenos de transporte de momento, calor $Y$ masa en la predicción de los perfiles de concentración y temperatura en el interior del reactor. La predicción de los perfiles de temperatura y concentración observados en reactores industrial y de escala piloto, se ha llevado a cabo con modelos pseudo-homogéneos $[15,16,19,31$ $33,87,125,144] \quad y$ pseudo-heterogéneos $[8,12,14,17,18,22,23,29,30,132-$ 134]. Actualmente, se sabe que el empleo de modelos pseudos-homogéneos, principalmente en una dimensión, solo sirven de forma exploratoria, teniendo como principal ventaja los tiempos de cómputo cortos. Para esta clase de reactores, cuando se emplean modelos pseudo-homogéneos en dos dimensiones, se considera la capacitancia de masa $y$ de calor si se trata de un estudio en estado transitorio. Cuando estos modelos se han empleado para predecir el comportamiento observado de un reactor industrial éstos fallan en la predicción de experimentos en estado estacionario y transitorio. Una extensa revisión de la literatura [6$8,11,14-16,23,87,133,19,144-173$ ] muestra que las principales fallas se relacionan con los fenómenos de desactivación del catalizador [8,12,13$16,18,23,25,29,31-34,133,134]$ y la sensibilidad de los perfiles de temperatura $Y$ concentración a los campos de velocidad $[30,41,43,58,61]$ y a los parámetros de transporte de calor $[14,15,87,88]$ (ver capitulos 2 y 3). Es importante considerar que las simulaciones empleando los modelos homogéneos 6 heterogéneos predicen los mismos resultados si se consideran diferentes valores de la magnitud de los parámetros de transporte de calor, o si se varía el perfil de desactivación a lo largo del lecho [14]. Con estos antecedentes, el presente trabajo va encaminado a esclarecer el efecto de los fenómenos hidrodinámicos, de transporte de calor y desactivación en la predicción del comportamiento observado en el reactor estudiado. 


\subsection{Catalizador}

El catalizador de $\mathrm{V}_{2} \mathrm{O}_{5} / \mathrm{TiO}_{2}$ ha sido caracterizado utilizando diversas técnicas analiticas (TPR-TPO, DRX, XPS, FT-IR, V-NMR, etc.), que han permitido un mejor entendimiento de las propiedades físico-químicas presentes en la superficie del catalizador $[11,15,16,35,146-173]$. Estas han sido relacionadas con la acidez 0 basicidad, con los tipos de oxígeno presentes en la superficie (nucleofilicos y electrofilicos) y con los estados de oxidación del vanadio. En la actualidad no se tiene claro cómo interaccionan los sitios activos del catalizador de $\mathrm{V}_{2} \mathrm{O}_{5} / \mathrm{TiO}_{2}$ con los hidrocarburos presentes, sin embargo, se han formulado varias propuestas.
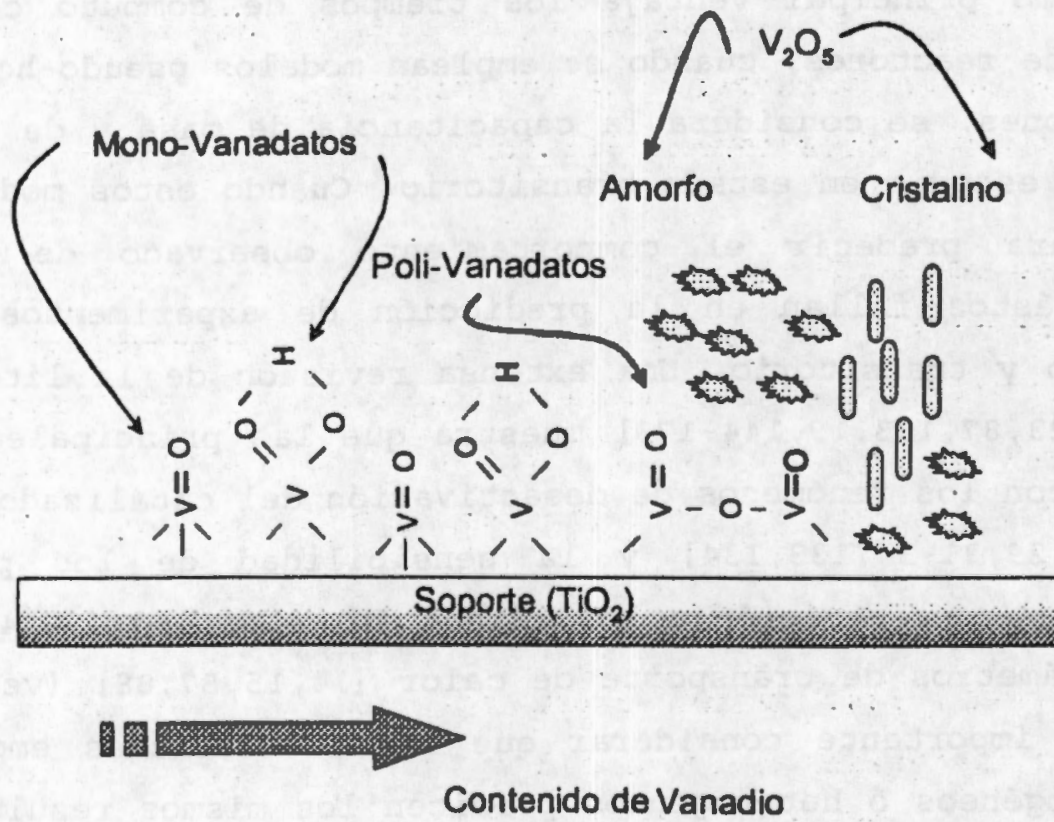

Figura 4.1. Los sitios presentes de $\mathrm{V}_{2} \mathrm{O}_{5}$ en la superficie del catalizador [149].

Algunos investigadores han encontrado que el vanadio se deposita en la superficie del Titanio en forma de capas, desde una monocapa hasta decenas de ellas, las cuales contienen principalmente especies monoméricas, poliméricas, cristalinas $y$ amorfas de vanadio [146-173]. En cuanto al desempeño de estas capas se ha reportado [146-173] que conforme mayor es la concentración de vanadio en la superficie de la 
Capitulo 4.

Estudio del comportamiento en estado estacionario del reactor de oxidación parcial de $\mathrm{OX}$ a $\mathrm{AF}$

titania, la selectividad a AF aumenta proporcionalmente; sin embargo, se han encontrado efectos contrarios $[150,153,154]$. Aparentemente, 10 anterior se puede atribuir a las diferentes metodologias de sintesis utilizadas por cada grupo de investigación $Y$, en menor grado, a las condiciones de evaluación del sరlido sintetizado. El catalizador que se utiliza en la industria contiene un poco más de una monocapa de vanadio con 4.8\% peso de este metal [168]. En la Figura 4.1 se muestra un esquema representativo de $10 s$ diferentes tipos de sitios activos que están presentes en el catalizador.

Algunas investigaciones $[11,146,149,152,155-157]$ consideran que el carácter reactivo del sistema $\mathrm{V}_{2} \mathrm{O}_{3} / \mathrm{TiO}_{2}$ esta relacionado con la presencia de sitios ácidos, Lewis y Bronsted, en la superficie. Los sitios Brensted se asocian con la superficie de la $\mathrm{TiO}_{2}$, y otros, de Lewis y Brensted con las capas de $\mathrm{V}_{2} \mathrm{O}_{5}$ [149]. Se propone que la combinación de un sitio fuerte Lewis con un exceso de oxígeno provocaría la ruptura de los anillos aromáticos, lo que daría como resultado la oxidación total $[11,149,157,158]$. Otras investigaciones [147,151] concluyen que dos tipos de sitios ácidos están presentes en su superficie, los débiles asociados con $V=0$ y los fuertes a $V-O-V$. Los sitios $V=0$ selectivos a la formación de $A F$, debido a la adsorción débil de ox $[147,151]$; sin embargo, se han reportado resultados contradictorios a éstos $[11,149,159,160]$.

En diversas publicacionea $[6,7,147,155,161-163]$ se concluye que el vanadio sobre la superficie de la $\mathrm{TiO}_{2}$ presenta diferentes estados de oxidación $\left(v^{+3}, v^{+4} y v^{+5}\right)$, los cuales tienen una fuerte influencia en el desempeño del catalizador. Se propone que la especie de $\mathbf{V}^{+5}$, que se encuentra en la parte superior de la capa, se reduce hasta $\mathrm{V}^{+3}$, lo cual de acuerdo con varios estudios $[7,11,147,149,157,158,161,162]$ se favorece en condiciones no oxidantes. Aparentemente la $\mathrm{TIO}_{2}$ no sufre una reducción importante. Los estudios sobre la evaluación del catalizador durante la oxidación de ox indican que al incrementar el contenido de $\mathrm{V}^{*}$ en las capas cercanas a la superficie se obtiene alta conversión ( $95 \%$ ) aunque una baja selectividad a AF (-35\%) [149]. Esto sugiere que 

reactor de oxidación parcial de OX a AF

el $\mathrm{V}^{+4}$ es el estado de oxidación selectivo para la oxidación total de ox a $\mathrm{CO}_{x}$. Sin embargo, al incrementar el contenido de $\mathrm{V}_{2} \mathrm{O}_{5}$ en la superficie de la $\mathrm{TiO}_{2}$, se obtiene un aumento en la selectividad a AF ( $\left.65 \%\right)$, para la misma conversión. Resultados semejantes se encontraron en los primeros estudios de Mars y van Krevelen (1954) [6] y Simard et al. (1955) [7]. Pocos estudios $[149,164]$ consideran que el $\mathrm{V}^{\text {t4 }}$ puede ser la fase activa para la oxidación parcial, mientras que otros [149,157,158,165] consideran que el buen desempeño del catalizador se debe a la presencia sitios $\mathrm{V}^{+4} \mathrm{y}^{+5}$ en la superficie del catalizador. Bond et al. (1998) [11,158] proponen un mecanismo de reacción, en donde sitios $\mathrm{V}^{+3}$ y $\mathrm{V}^{+5}$ son los estados de oxidación selectivos para la. quimisorción de las moléculas aromáticas en la superficie del catalizador. Si otro sitio $\mathrm{V}^{+3}$ se encuentra cerca, se tendrá una adsorción fuerte de la molécula aromática, la cual tiende a polimerizarse y tener una deshidrogenación. Parte de estas moléculas aromáticas, principalmente el $\mathrm{OX}$, son convertidas a $\mathrm{CO}$ y $\mathrm{CO}_{2}$, mientras que si un sitio $\mathrm{V}^{+5}$ se encuentra cerca de la molécula adsorbida se tendrá como resultado la oxidación parcial del hidrocarburo.

Un grupo de investigadores $[152,164,166-171]$, con una hipotesis totalmente diferente a las anteriores, al realizar estudios transitorios de la oxidación parcial de Tolueno, concluyen que el catión $\mathrm{V}^{+4}$ es el sitio que da origen a la formación de dos estados $\mathrm{V}^{+5}$, uno conteniendo oxígeno de carácter electrofilico el cual es el responsable de llevar a cabo la oxidación total del anillo aromático, y el otro conteniendo oxígeno con carăcter núcleofilico, que lleva a cabo la oxidación parcial del anillo aromático.

La mayoría de los estudios para evaluar los catalizadores, nunca se ha llevado a cabo en un reactor industrial, ya que esto implica un nivel de incertidumbre importante en la interpretación del proceso cinêtico. Por lo que, una combinación entre los estudios cinéticos realizados a nivel laboratorio $y$ el estudio del comportamiento del reactor industrial a las correspondientes condiciones de operación, es decir, temperatura del baño de enfriamiento $T_{b}$. Temperatura de entrada $T_{0} Y$ 

reactor de oxidación percial de OX a AF

concentración de entrada del reactante al reactor $C_{o x}$ pidiera dar interesante información sobre el sistema de estudio.

\subsubsection{Papel del oxigeno durante la reacción}

Actualmente existe una gran controversia sobre la explicación de la posible interacción entre el oxígeno y el catalizador. Gracias a los trabajos de diversos autores $[11,14,15,149,157,158,166-171]$ se sabe que el oxigeno adsorbido en el catalizador mantiene un equilibrio dinámico con el oxígeno de la fase gaseosa durante la reacción. Durante este equilibrio dinámico se tiene la regeneración parcial de los sitios activos, que son selectivos a la oxidación parcial del ox. Durante el transcurso de la reacción hay una relación directa entre la dinámica oxido-reducción del catalizador y su desempeño catalitico.

La dispersión del vanadio sobre la superficie de la titania es uno de los principales factores que controlan la interacción del oxígeno con la superficie $y$, consecuentemente, su desempeño catalitico. Diversos estudios proponen $[132,149,157,158,165-171]$, con base en un análisis de TPR/TPO, que el oxigeno presente en los grupos terminales $V=0$ es más lábil que el oxigeno que forma 108 puentes en 108 enlaceg $\mathrm{V}-\mathrm{O}-\mathrm{V}$ o $\mathrm{V}-\mathrm{O}$ Ti. Por ende el grupo $V=0$, además de provocar una adsorción débil con la superficie, su oxígeno lábil. sirve tanto para llevar a cabo la oxidación parcial de los hidrocarburos presentes como la re-oxidación de algunos sitios que se encuentran reducidos.

Diversos trabajos $[15,19]$, mencionan que la oxidación de los sitios selectivos a la oxidación parcial es muy rápida, esto se debe a que la caracterización del catalizador empleado en un reactor a escala piloto. no muestra la presencia de estados de oxidación bajos en la superficie del catalizador [15]. Por otro lado, la caracterización por TGA realizada a este mismo catalizador, muestran la presencia de hidrocarburos fuertemente químiadsorbidos $y$ deshidrogenados en la superficie del catalizador, aparentemente siendo estos los responsables 
de la pérdida de actividad del catalizador durante la reacción $[11,157,158,168]$.

\subsubsection{Fenómenos de desactivación en la superficle del catalizador}

En la literatura $(11,14,16,18,19,157,158]$ se ha reportado que los catalizadores de vanadio empleados en reacciones de oxidación parcial se desactivan de forma reversible e irreversible. La primera se ha asociado a una fuerte reducción del vanadio como consecuencia de una operación prolongada a alta tempęraturas y alta concentración de hidrocarburo. La segunda ha sido vinculada con cambios estructurales en el catalizador, un cambio de fase del $\mathrm{TiO}_{2}$, de Anatasa a Rutilo, lo cual se relaciono con las altas temperaturas en la posición del punto caliente. Este ultimo tipo de desactivación se ha cuestionado $[15,19]$, ya que caracterizaciones por difracción de Rayos $X$ (DRX), que se realizaron a un catalizador industrial sometido a reacción por más de 5 años presento un cambio minimo de fase de la $\mathrm{TiO}_{2}$, lo que ha dado lugar a investigar otras posibles fuentes de desactivación.

Lo anterior sugiere que, para el sistema $\mathrm{V}_{2} \mathrm{O}_{5} / \mathrm{TiO} \mathrm{O}_{2}$, existen dos tipos de perfiles de actividad. El primero es un perfil de óxido-reducción, el cual se conoce como reversible $y$ requiere de un cierto tiempo para ajustarse a diferentes condiciones de operación. Este perfil de actividad considera una adsorción de moléculas de hidrocarburos en la superficie del catalizador, las cuales provocan que sitios selectivos a la oxidación parcial no sean re-oxidados durante la reacción $[11,15,16,19,157,158,168]$. Se considera como reversible, porque la oxidación a altas temperaturas del catalizador por varias horas, con aire $y$ en ausencia de hidrocarburo, restablece el nivel original de actividad del catalizador $[14,15]$. Por esto, el uso de una atmósfera oxidante incrementa la actividad y selectividad a AF, mientras que una atmósfera reductora disminuye estas propiedades [11,14$16,19,157,158,168]$. Conforme el tiempo operación del reactor avanza (meses $\sigma$ años), se tiene una fuerte adsorción de las moléculas de 

reactor de oxidación parcial de OX a AF

hidrocarburo (OX) en la superficie catalizador, desactivando de forma gradual la superficie del catalizador. Este efecto se puede ver en los perfiles de temperatura observados en un reactor industrial [14-16] .

El segundo perfil considera una superficie que ha sido sometida a condiciones drásticas de reacción por varios meses (hasta años), principalmente a altas temperaturas $y$ altas concentraciones de hidrocarburo alrededor de la zona del punto caliente. Este perfil se podría deber a dos motivos $[14-16]$ : 1) en menor grado al cambio de la fase de la $\mathrm{TiO}_{2}$ y 2 2) muy probablemente a la fuerte adsorción de hidrocarburos polimerizados en la superficie del catalizador, lo que da lugar a la formación de "coque" por un fenómeno de polimerización y deshidrogenación. Experimentos en el reactor industrial [14-16], muestran que este coque no puede ser totalmente oxidado a $\mathrm{CO}$ y $\mathrm{CO}_{2}$ bajo las condiciones normales de re-oxidación del catalizador con aire $y$ una temperatura de $683 \mathrm{~K}$ por más de $20 \mathrm{~h}$; lo cual ocasiona que la actividad del catalizador no se vea completamente recuperada, este fenómeno se observa cuando el reactor ha sido operado por meses o hasta años. Los experimentos transitorios en un reactor a escala industrial $[14,18,19]$, han mostrado que importantes incrementos de la temperatura del baño, bajo condiciones de reacción, pueden hacer que parte de estos hidrocarburos se eliminen de la superficie del catalizador, reactivando parcialmente la zona desactivada (que se pensaba estaba desactivada irreversiblemente) del lecho. Lo anterior sugiere que durante la etapa de re-oxidación del catalizador deben utilizarse condiciones más severas (mayores temperaturas del baño y tiempos de flujo de aire) conforme se incrementa su tiempo de uso. Tendiendo en mente que si la conversion $y$ selectividad no se ven alteradas, como ha sido observado en algunos estudios [14,16], este fenomeno de desactivación se puede emplear favorablemente para no tener altas temperaturas en la posicion del punto caliente y asi evitar el probable cambio de fase de la. $\mathrm{TiO}_{2}$.

Lo anterior muestra que la actividad del catalizador presenta cambios sustanciales con el tiempo de reacción durante largos periodos de operación; sin embargo, el perfil de actividad no es una cantidad que 

reactor de oxidación parcial de OX a AF

pueda ser medida en sí misma, aunque en un principio este se puede inferir de mediciones de temperatura y concentracion. Por esto, es necesario proponer un modelo que describa la actividad del catalizador, tomando en cuenta la desactivación reversible, por ejemplo un modelo mecánistico, e irreversible, por ejemplo una ecuación empirica, del catalizador.

\subsection{Estudios cinéticos en la actualidad}

El modelo cinético propuesto por Mars y van Krevelen [6] está basado en un mecanismo de óxido-reducción (redox). Aqur el hidrocarburo aromático reduce la superficie del catalizador y a su vez el sitio reducido es oxidado con el oxígeno proveniente de la fase gas. Aunque recientemente se cuestiona a este modelo cinético [173], sus ideas sobre la dinámica redox en la superficie del catalizador se siguen empleando. Simard et al. [7] llega a conclusiones semejantes a las de Mars $y$ van Krevelen [6]. Esta hipotesis ha entrado en conflicto con otros estudios $[15,19]$, ya que se ha encontrado que la re-oxidación del catalizador de vanadio de $\mathrm{V}^{+3}$ a $\mathrm{V}^{+5}$ es muy rápida, como consecuencia de esto, otro mecanismo de reacción (por ejemplo del tipo Langmuir-Hinshelwood que considere la adsorción de los hidrocarburos y oxigeno como paso de reacción controlante) tendría que ser propuesto $[17,19]$.

La Figura 4.2 y la Tabla 4.1 muestran los esquemas de reacción y las etapas de reacción publicados a la fecha para la oxidación de OX a AF, respectivamente. Una revisión completa de los diferentes estudios cinéticos se puede consultar en las referencias $[25,35,149,172]$. La mayoría de los modelos cinéticos se evaluaron bajo condiciones de operación diferentes a las que se tienen en el reactor a escala industrial; principalmente aquéllas que tienen lugar en la primera parte del reactor, es decir, alrededor del punto caliente. Esto ocasiona que se tengan predicciones diferentes a las que suceden en el reactor industrial, en donde se tienen altas concentraciones de hidrocarburos $y$ altas temperaturas e importantes fenómenos de 

reactor de oxidación parcial de OX a AF

desactivación toman lugar. Contrario a esto, el estudio cinético desarrollado por Calderbank et al. [8] utilizo las condicione de operación que se trabajan en el reactor industrial para evaluar su modelo cinético. Sin embargo este modelo cinético ha mostrado tener algunas desventajas en la predicción de los datos experimentales $[14,15,18,19,23,133,134]$, ya que no incorpora la desactivación del catalizador y ni la formación del anhídrido maleico (AM); no obstante, sólo este modelo cinético ha sido empleado para simular el comportamiento observado de un reactor industrial $[14,15,18,19,23,133,134]$.

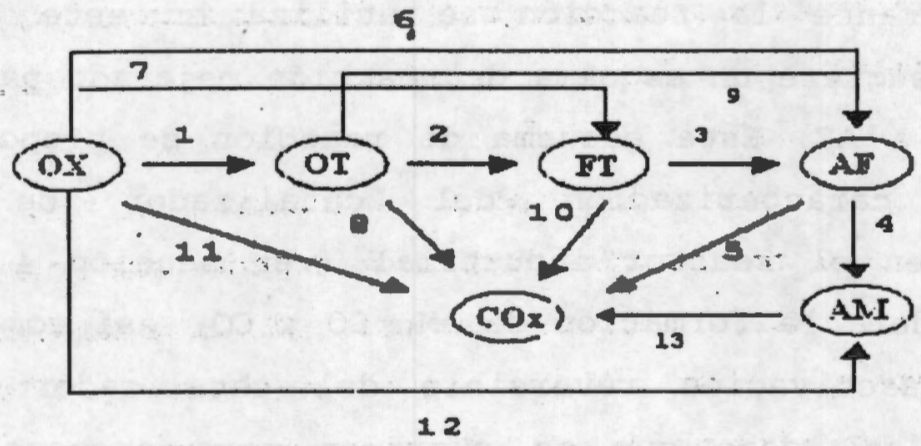

Figura 4.2. Esquemas cinéticos de la oxidación de ox a AF publicados por varios investigadores (ver Tabla 4.1).

Tabla 4.1. Diferentes etapas de reacción considerados en la literatura, de acuerdo a la Figura 4.2

\begin{tabular}{|c|c|c|c|c|c|c|c|c|c|c|c|c|}
\hline Etapa de reacción & 1 & 2 & 3 & 4 & 5 & 6 & 7 & 8 & 9.10 & 11 & 12 & 13 \\
\hline Simard $y$ et al.. 1956 & $\mathrm{x}$ & & & & & $\bar{x}$ & & & $\mathrm{x}$ & $\bar{x}$ & $\bar{x}$ & \\
\hline Lyubarskii et al., 1973 y 1974 & $x$ & & & & & $\mathrm{x}$ & & & $x$ & $x$ & $x$ & \\
\hline Costa et al., 1963 & $x$ & & & & & & & $x$ & $\mathrm{x}$ & $\mathbf{x}$ & $\mathbf{x}$ & $\mathbf{x}$ \\
\hline Boag et al., 1975 & $x$ & $\mathbf{x}$ & & & & $x$ & $x$ & $x$ & $\mathrm{x}$ & $\mathbf{x}$ & & \\
\hline Herten y Froment, 1968 & $x$ & $\mathrm{x}$ & $\mathbf{x}$ & & $x$ & $\mathbf{x}$ & $\mathbf{x}$ & $x$ & $\mathrm{x} x$ & & & \\
\hline Ellis 1972 & $x$ & $\mathbf{x}$ & $\mathrm{x}$ & & $\mathrm{x}$ & & & & & $x$ & & \\
\hline Skrzypek et al., 1985 & $x$ & $x$ & $x$ & $x$ & $x$ & $x$ & & $\mathbf{x}$ & & $\mathbf{x}$ & & \\
\hline Papageorgiou et al., 1994 & $x$ & $\mathrm{x}$ & $x$ & & $\mathrm{x}$ & $x$ & & $\mathrm{x}$ & & $\mathbf{x}$ & & \\
\hline Wainwright et al., 1977 & $x$ & $x$ & $x$ & & & & & $\mathbf{x}$ & & $\mathrm{x}$ & & \\
\hline Calderbank et al., 1977 & $x$ & $x$ & $x$ & & $x$ & $\mathrm{x}$ & & & & $\mathrm{x}$ & & \\
\hline Chandraekharan et al.. 1979 & $x$ & & & & & $\mathbf{x}$ & & & $\mathrm{x}$ & $x$ & & \\
\hline Froment et al.. 1994 & $x$ & $\mathbf{x}$ & $\mathrm{x}$ & & & & & & $x$ & $\mathrm{x}$ & & \\
\hline
\end{tabular}


En el Apéndice $\sharp$ se evalúan los modelos cinéticos de Calderbank et al. [8], Skrzypek et al. [17] y Papageorgiou et al. [132] para predecir los perfiles de temperatura $Y$ concentración observados en el reactor industrial a la condiciones del Exp-1 (ver Tabla 4.3). Estos resultados $y$ aquéllos expuestos por diversos autores [19,23] sugieren que el modelo cinético de Calderbank et al. [8] es la mejor alternativa a la fecha para predecir el comportamiento cinético del catalizador industrial de $\mathrm{V}_{2} \mathrm{O}_{5} / \mathrm{TiO}_{2}[19,23]$, en la operación del reactor en estado estacionario. Si bien el modelo cinético de Calderbank et al. [8] no considera la desactivación reversible e irreversible que sufre el catalizador durante la reacción se utiliza en este estudio. En el Apéndice I se sugiere un esquema de reacción mejorado para la oxidación parcial de OX a AF. Este esquema de reacción se propone a partir de estudios de caracterización del catalizador de $\mathrm{V}_{2} \mathrm{O}_{5} / \mathrm{TiO}_{2}$, $\mathrm{y}$ observaciones en el reactor industrial (ver sección 4.5). El esquema cinético considera la formación de $\mathrm{AM}, \mathrm{CO}$ y $\mathrm{CO}_{2}$, asi como una etapa que explica la desactivación reversible del catalizador. Es importante destacar que las ideas que se sugieren para proponer este esquema cinético se emplean en la discusión del estụdio con reacción en estado estacionario y transitorio.

\subsection{Sistema experimental}

Los datos experimentales que se emplean en este trabajo fueron tomados de los trabajos de López-Isunza [14] y Mongkhonsi [15]. En ambos trabajos el reactor es el mismo, en tanto que la única diferencia reside en 10 equipos periféricos empleados. La Figura 4.3 muestra el diagrama de flujo de la planta piloto donde se efectuó la oxidación parcial de $O X$ a AF. La Tabla 4.2 muestra las caracteristicas geométricas del reactor y algunas propiedades físicas del catalizador empleado. 
Tabla 4.2. Caracteristicas del reactor y

del catalizador

\begin{tabular}{|c|c|}
\hline Reactor & Valor \\
\hline Longitud, m & 3.350 \\
\hline Diámetro, m & 0.025 \\
\hline Espesor de la pared, m & 0.0025 \\
\hline Lecho empacado, $\mathrm{m}$ & 2.6 \\
\hline Densidad del lecho empacado, $\mathrm{kg} \cdot \mathrm{m}^{-3}$ & 1312 \\
\hline \multicolumn{2}{|l|}{ Catalizador } \\
\hline Dismetro, m & 0.008 \\
\hline Densidad*, $\mathrm{kg}_{\text {e611ido }} \cdot \mathrm{m}^{-3}$ s61ido & 2000 \\
\hline Capacịdad calorifica especifica*, $\mathrm{kJ}(\mathrm{kg} \cdot \mathrm{K})^{-1}$ & 0.1047 \\
\hline Conductividad Térmica*, W(m K) ${ }^{-1}$ & 0.1047 \\
\hline
\end{tabular}

La mezcla de reacción estuvo compuesta de 1 mol de ox en aire a condiciones normales de temperatura y presión (298 K y 1 atm). El OX antes de alimentarse al reactor es vaporizado a $378-383 \mathrm{~K}$. El OX vaporizado es. arrastrado por el aire que se alimenta al reactor. El reactor consta de un tubo de $0.025 \mathrm{~m}$ de diámetro interno y $3.35 \mathrm{~m}$ de longitud, en donde se empacan $2.5 \mathrm{~m}$ de catalizador esférico (no poroso de $\mathrm{V}_{2} \mathrm{O}_{5}$ externamente depositado en $\mathrm{TiO}_{2}$ / fabricado por Chemische Frabrik von Heyden. El reactor se encuentra sumergido en un baño de sal fundida conteniendo una mezcla de $59 \%$ de nitrato de potasio con $41 \%$ de nitrato de sodio, el cual provee la temperatura necesaria para que tenga lugar la reacción y actúa como medio de enfriamiento. El gas que sale del reactor pasa a través de un condensador donde el AF se sublima. Los gases remanentes son lavados con agua en una torre empacada para eliminar los compuestos orgánicos y el gas restante se ventea a la atmósfera. 


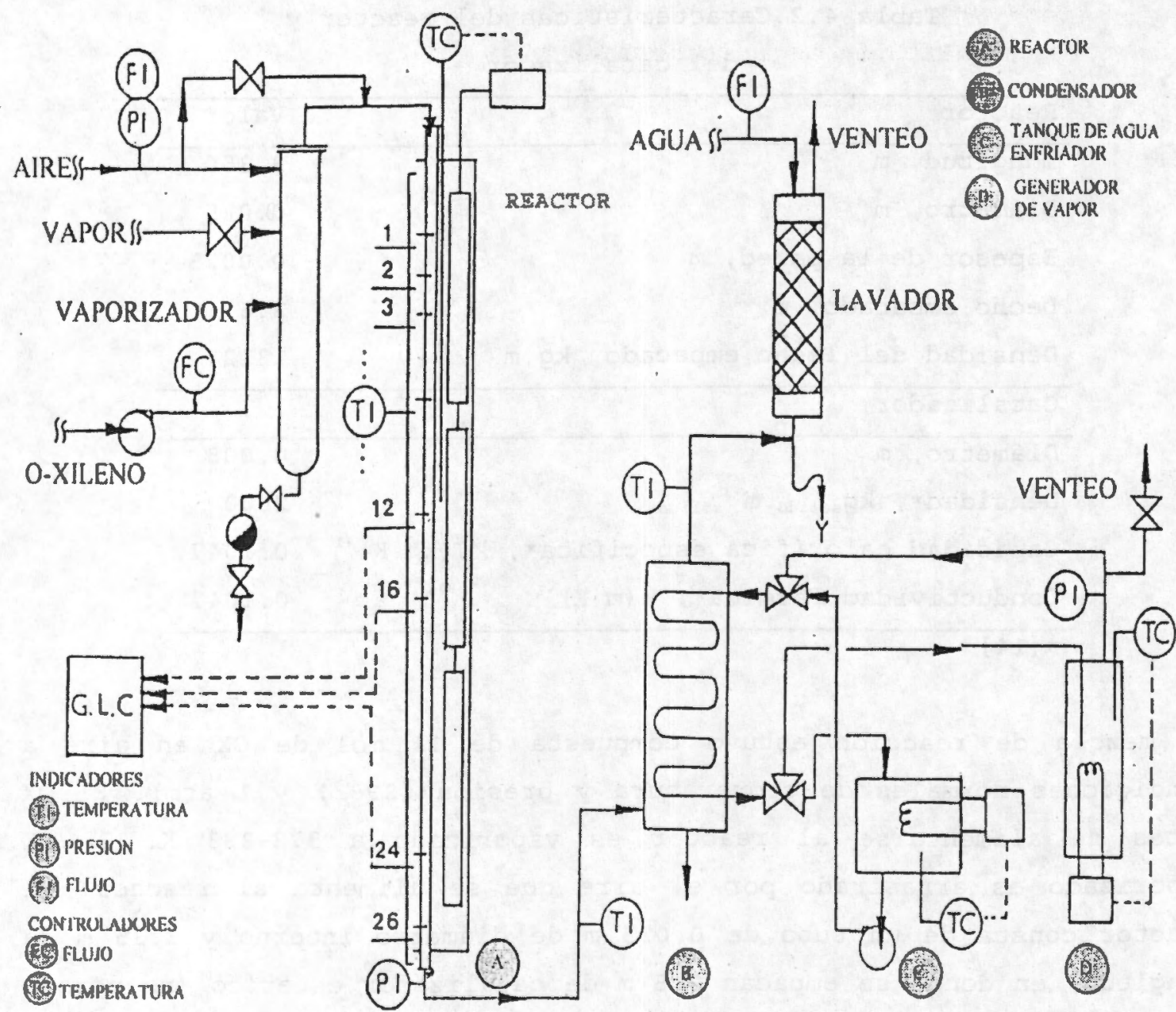

Figura 4.3. Diagrama de la planta piloto para la oxidación parcial de OX a AF [14].

A lo largo del reactor se tienen 26 termopozos para medir la temperatura, $y$ en cinco de ellos muestrear los gases de reacción. La determinación de la composición de hidrocarburos es en línea, mediante cromatografía de gases. Los termopares se pueden desplazar libremente a lo largo de la posición radial. Sin embargo, los termopozos provocaron la distorsión de los perfiles de temperatura radiales, ya que proporcionaron un área adicional de transporte de calor. Más detalles sobre el sistema experimental se puede encontrar en los trabajos de López-Isunza [14] y Mongkhonsi [15] . 


\subsection{Experimentos y resultados en el reactor}

Ia Tabla 4:3 muestra las condiciones de operación del reactor correspondientes a los casos de estudio en este capitulo y que consideran la simulación del reactor en estado estacionario. Estos casos fueron seleccionados ya que son los que se manejan en el reactor industrial. Las Figuras 4.4-4.6 muestran los perfiles de temperatura y concentración observados en el reactor industrial a diferentes condiciones de operación junto con los diferentes grados de desactivación del catalizador. La Figura 4.4a muestra la comparación de los perfiles de temperatura axiales a las condiciones de los experimentos Exp-1 y Exp-1-3. La Figura 4.4.b muestra los perfiles de temperatura radiales únicamente a las condiciones del Exp-1. La Figura 4.5 muestra 10 perfiles axiales de concentración de OX y AF a la condiciones del Exp-1 y la Figura 4.6 presenta los perfiles de temperatura que se registran a diferentes tiempos de reacción a las condiciones de operación del Exp-3. En este experimento el catalizador tiene alrededor de una semana de uso.

Tabla 4.3. Condiciones experimentales

\begin{tabular}{|c|c|c|c|}
\hline Vardable & $\operatorname{Exp}-1[14]$ & $\operatorname{Exp}-2$ [15] & $\operatorname{Exp}-3[14]$ \\
\hline $\begin{array}{l}\text { Temperatura del baño } \\
\text { de sal, } K\end{array}$ & 661 & 676 & 649 \\
\hline $\begin{array}{l}\text { Temperatura de } \\
\text { Alimentación, } \mathrm{K}\end{array}$ & 463 & 463 & 565 \\
\hline $\begin{array}{l}\text { ox alimentado, } \mathrm{g} \cdot \mathrm{h}^{-1} \\
\text { (1tmol en aire) }\end{array}$ & 176 & 176 & 176 \\
\hline Flujo volumétrico de & 4 & 4 & 4 \\
\hline $\begin{array}{l}\text { aire alimentado al } \\
\text { reactor, } \mathrm{m}^{3} \cdot \mathrm{h}^{-1} \quad(\mathrm{P}=1 \\
\text { atm y } 298 \mathrm{k})\end{array}$ & & & \\
\hline $\begin{array}{l}\text { Flujo másico de } \\
\text { aire, } \mathrm{kg}\left(\mathrm{m}^{2} \mathrm{~h}\right)^{-1}\end{array}$ & 10548 & 10548 & 10548 \\
\hline
\end{tabular}



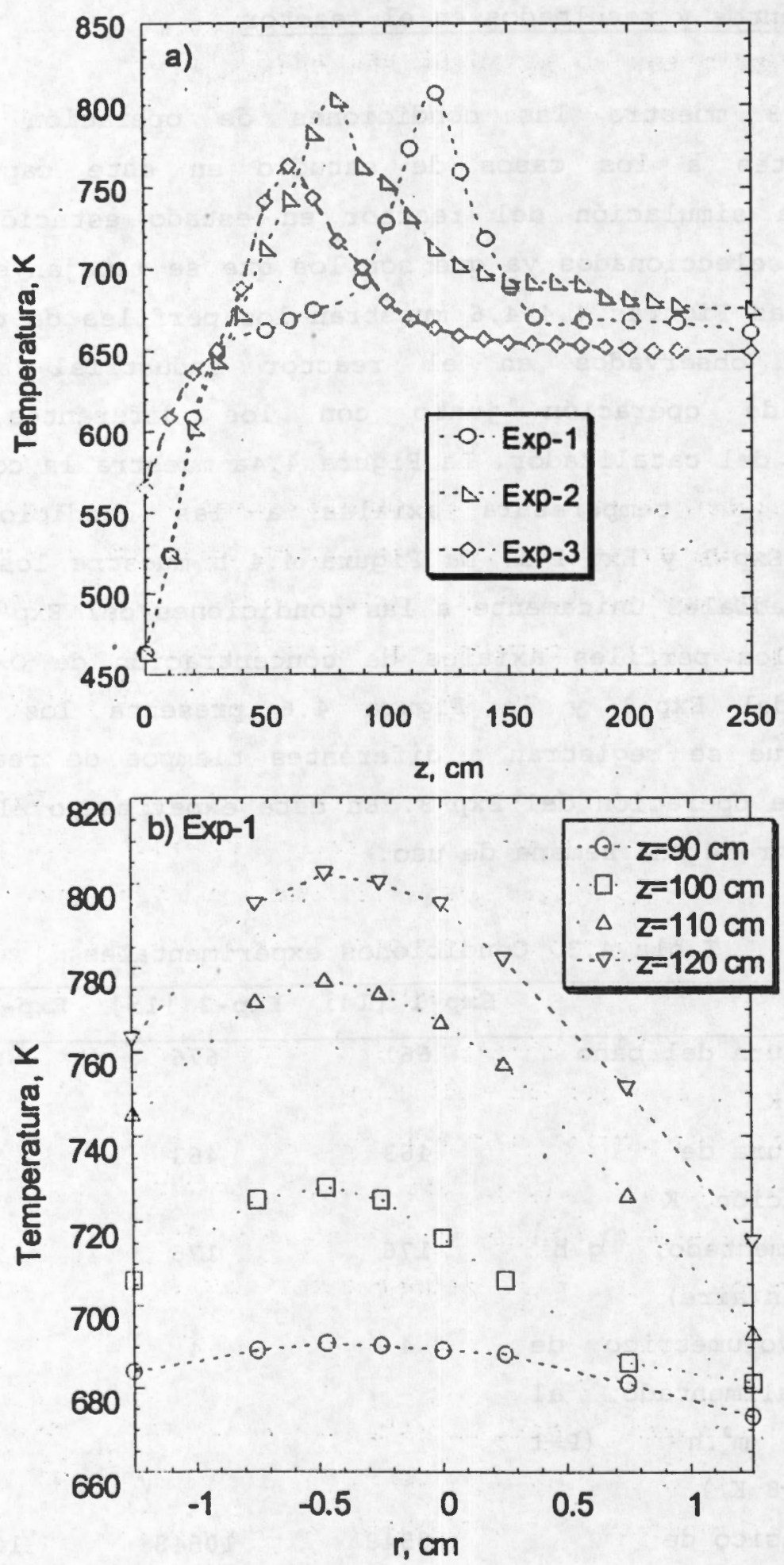

Figura 4.4. Perfiles de temperatura en el reactor alanzados desde el arranque al primer estado estacionario. a) Axial y b) radial [14]. 


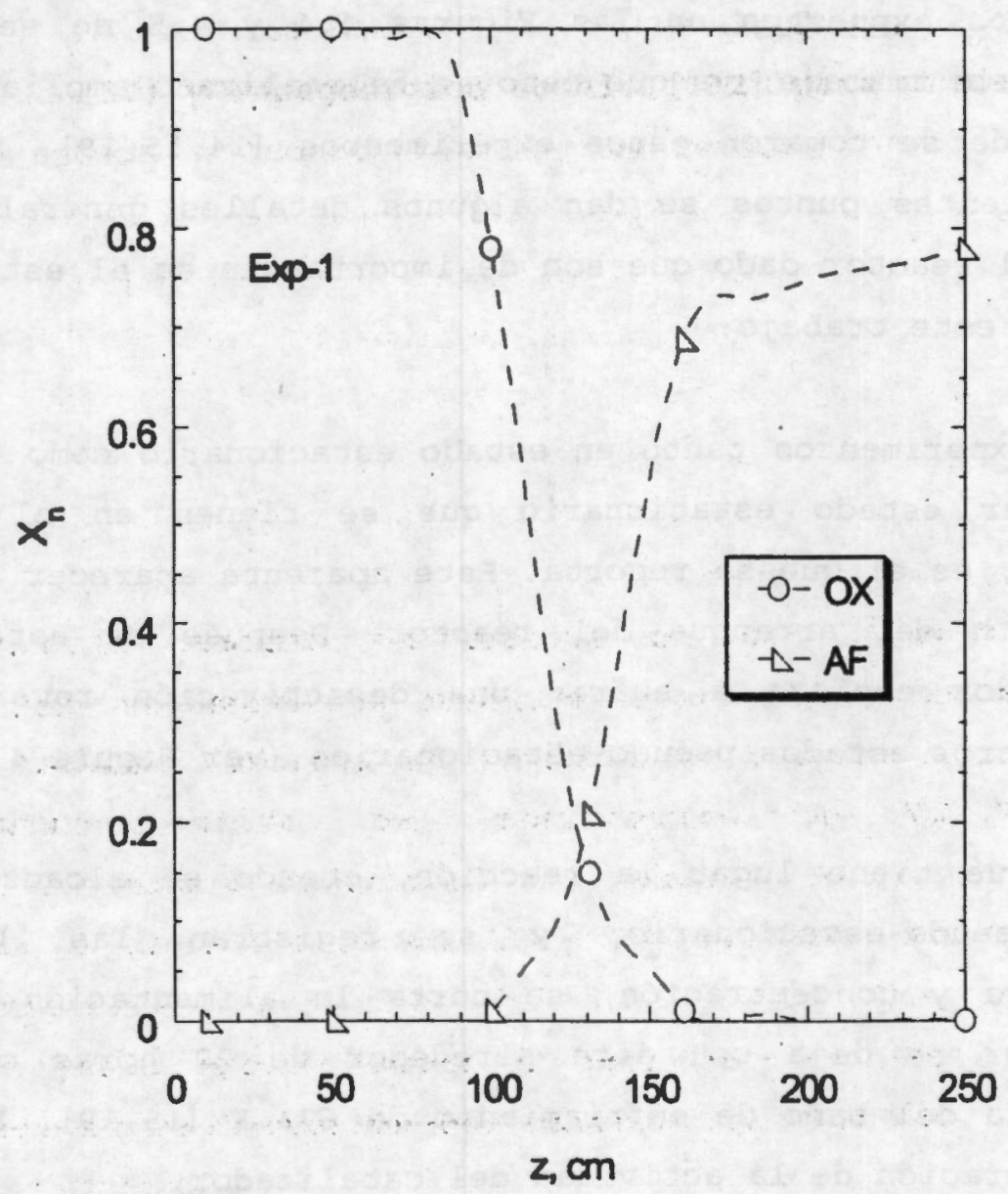

Figura 4.5. Los perfiles de concentración de OX y AF para las condiciones del Exp-1.

En este trabajo se emplean los experimentos Exp-1 y Exp-3 para estudiar los siguientes puntos:

1. - El efecto de la desactivación del catalizador en la capacidad de predicción de las observaciones que se tienen en el reactor. Asimismo, se estudia la capacidad de predicción del modelo del reactor al considerar diferentes fenómenos de transporte de momento calor y masa en el reactor, ver la sección 4.7.

2. -El impacto de los parámetros transporte de calor, $h_{w} \mathbf{y} k_{\text {er, }}$ estimados sin reacción (ver el Capitulo 3), en la capacidad de predicción en el modelo del reactor, ver la sección 4.8 . 
Capitulo 4.

Estudio del comportamiento en estado estacionario del reactor de oxidación parcial de OX a AF

Los resultados expuestos en las Figuras 4.4 y 4.5 no se discuten a detalle en este trabajo, ya que esto se ha realizado ampliamente en los trabajos donde se tomaron estos experimentos [14,15,19]. Sin embargo, en los siguientes puntos se dan algunos detalles generales sobre la operación del reactor dado que son de importancia en el estudio teórico realizado en este trabajo:

a) En los experimentos tanto en estado estacionario como transitorio, el primer estado estacionario que se tienen en el sistema de reacción, es el que se reporta. Este aparenta aparecer alrededor de las $0.7 \mathrm{~h}$ del arranque del reactor. Después de este tiempo el catalizador empieza a sufrir una desactivación reversible $y$ se tienen otros estados pseudo-estacionarios (ver Figura 4.6).

b) Una vez que tiene lugar la reacción, cuando se alcanza el primer estado pseudo-estacionario, $y$ se registran las lecturas de temperatura y concentración, se corta la alimentación de ox y el catalizador se deja con aire alrededor de 20 horas o más a una temperatura del baño de enfriamiento de $673 \mathrm{~K}[15,19]$. Esto permite una regeneración de la actividad del catalizador.

c) Los perfiles radiales de temperatura (ver Figura 4.4b) presentan no-simetría como una consecuencia de la pérdida de calor a lo largo del termopar-termopozo. El modelamiento del reactor considera el lado izquierdo del perfil de temperatura radial, ya que da valiosa información de los procesos de transporte de calor y masa.

d) Los perfiles radiales de temperatura observados en el reactor (ver Figura 4.4b) muestran que la diferencia de temperatura entre la superficie del catalizador $y$ el gas que la rodea no es mayor a $10^{\circ}$ [14]. Esto se observó ya que a una misma posición axial, las lecturas de temperatura, entre dos posiciones radiales adyacentes, nunca se increment 6 considerablemente. 

reactor de oxidación parcial de $\mathrm{OX}$ a $\mathrm{AF}$

La Figura 4.6 muestra los perfiles de temperatura axiales desde el arranque del reactor hasta las 38 horas de reacción, los cuales no han sido reportados en la literatura. Estos muestran principalmente el efecto de la desactivación reversible que sufre el catalizador y se discuten en los siguientes puntos:

a) El primer estado estacionario se presenta desde $0.7 \mathrm{~h}$ hasta las primeras $19 \mathrm{~h}$ de reacción. Durante este intervalo de tiempo se observan variaciones pequeñas entre los perfiles axiales de temperatura como consecuencia de variaciones en la presión del condensador debidos a la sublimación de algunos productos. Después de este tiempo, el perfil de temperatura se desplaza hacia la salida del reactor $(-50 \mathrm{~cm})$, hasta alcanzar un nuevo estado estacionario (30-38 h). Esta región de operación, de acuerdo a trabajos publicados $[23,133,134]$, se tiene en el reactor industrial.

b) La magnitud del punto caliente, $715 \mathrm{~K}$ a $\sim 110 \mathrm{~cm}$, a las $38 \mathrm{~h}$ es $40^{\circ}$ menor del observado a las $19 \mathrm{~h}, 755 \mathrm{~K}$ A $-60 \mathrm{~cm}$. Esto podría deberse a un fenómeno de desactivación reversible del catalizador a la entrada del reactor. Lo cual se relaciona con la dosificación de la reacción a lo largo del reactor, asi la concentración de ox que llega a la superficie del catalizador cercana al punto caliente a las $38 \mathrm{~h}$ es menor a aquélla que se tenía a la entrada del lecho a las $19 \mathrm{~h}$. Lo anterior se observa cuando se compara el perfil de temperatura con reacción con aquel que presenta únicamente un proceso de transporte de calor, en donde después de los primeros 20 $\mathrm{cm}$, cuando se tiene una temperatura de $630 \mathrm{~K}$, siempre tiene lugar la reacción.

c) Un experimento independiente a éste, reportado por Lopez-Isunza [14], muestra que la producción total de AF es similar (67-75\%), no importando el perfil de temperatura o el nivel de desactivación que sufre el catalizador a la entrada del lecho. Esto se debe a que el AF se produce despues de la posición del punto caliente y necesita solamente de una corta distancia de la longitud del reactor para 

reactor de axidación parcial de $\mathrm{OX}$ a AF

alcanzar su máximo rendimiento (ver Figura 4.5). Ya que esta zona debe estar rica en sitios selectivos a la oxidación parcial (ver Apéndice I) [11].

Estas observaciones no son empleadas en el estudio teorico del reactor, sin embargo, se utilizan en la propuesta del esquema de reacción que se presenta en el Apéndice $I$.

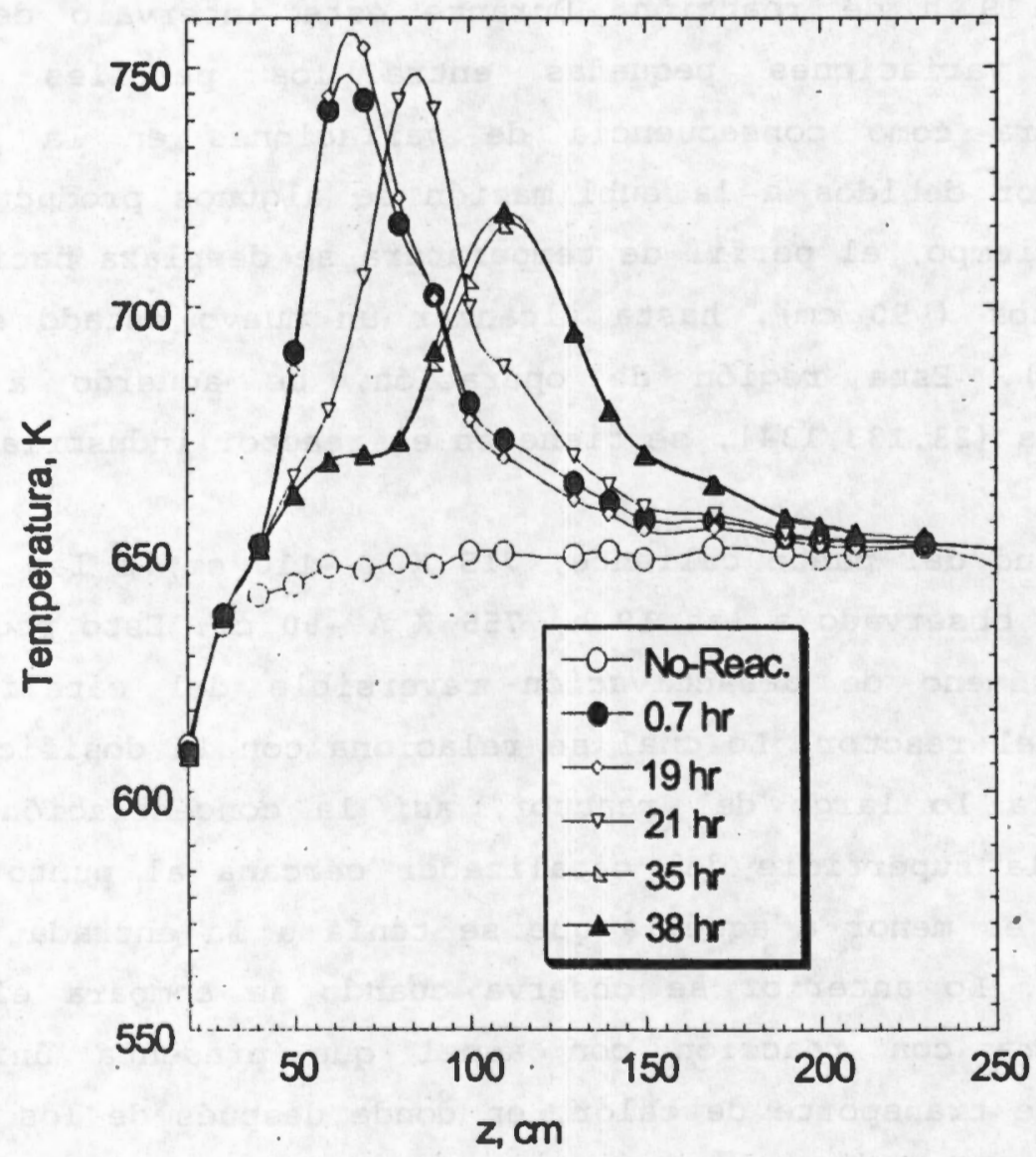

Figura 4.6. Perfiles de temperatura a las condiciones del Exp-3, a diferentes tiempos de reacción.

\subsection{Modelos del rector empleados en el presente estudio}

La finalidad de emplear el modelo del reactor con diferentes niveles de complejidad es observar su capacidad de predicción de los experimentos 

reactor de oxidación parcial de $\mathrm{OX}$ a AF

mostrados en las Figuras 4.5 y 4.6, que corresponden a las condiciones de operación de los experimentos 1-3. En la simulación del reactor se estudia el efecto tanto de los procesos de transporte de momento calor y masa, como de la desactivación del catalizador. En los siguientes puntos se describe brevemente cada uno de los modelos empleados para predecir el comportamiento del reactor tanto en el estado estacionario como en el transitorio.

\subsubsection{Modelo pseudo-homogéneo en una dimensión espacial}

El modelo homogéneo en una dimensión espacial (HOM-1D) considera que la concentración de las distintas especies y la temperatura varian unicamente en la dirección axial. El transporte de masa y calor por convección es el mecanismo dominante. Normalmente los fenómenos de dispersión axial son depreciados, sin embargo, aqui se consideraran debido a la magnitud de los gradientes axiales de temperatura que se tienen. El modelo que describe esta situación física está dado por las siguientes ecuaciones:

$\varepsilon \frac{\partial C_{n}}{\partial t}+V_{0} \frac{\partial C_{n}}{\partial z}=\varepsilon D_{e z} \frac{\partial^{2} C_{n}}{\partial z^{2}}+\rho_{B} \sum_{j=1}^{N} v_{n j} R_{f}$

$\rho_{B} C_{p s} \frac{\partial T}{\partial t}+V_{o} \rho_{z} C_{p f} \frac{\partial T}{\partial z}=k_{e z} \frac{\partial^{2} T}{\partial z^{2}}+\frac{4 U}{d_{t}}(T-T)+\rho_{B} \sum_{j=1}\left(-\Delta H_{j} R_{j}\right.$

Las condiciones inicial y de frontera son:

En $t=0 ; \quad C_{n}(0, z)=C_{n}(0, z)_{\text {ss }}$

$$
T(0, z)=T(0, z) s s
$$



reactor de oxidación parcial de OX a AF

$$
\begin{array}{ll}
\text { En } z=0 ; \quad V_{0} C_{n o}=V_{0} C_{n}-\varepsilon D_{e z} \frac{\partial C_{n}}{\partial z} \\
V_{o} \rho_{\varepsilon} C_{p t} T_{0}=V_{o} \rho_{\varepsilon} C_{p t} T-k_{e z} \frac{\partial T}{\partial z} \\
\text { En } z=L ; \quad \frac{\partial C_{n}}{\partial z}=0 \\
\frac{\partial T}{\partial z}=0
\end{array}
$$

\subsubsection{El modelo pseudo-heterogéneo en una dimensión espacial}

En reacciones altamente exotérmicas, en donde se tienen. importantes resistencias interfaciales, es importante hacer la distinción entre la fase sólida y gas debido al efecto de la temperatura en la desactivación irreversible del catalizador. En operaciones del reactor en estado transitorio es conveniente emplear un modelo pseudoheterogéneo, ya que la reacción tiene lugar en el catalizador y los procesos de transporte de calor son controlados por éste. En el modelo pseudo-heterogéneo en una dimensión espacial (HET-1D) hace la distinción entre la fase sólida y el gas (ver la Figura 4.7). Las ecuaciones de masa y calor del sólido y el gas se unen a través del transporte en la interfase sólido-gas.

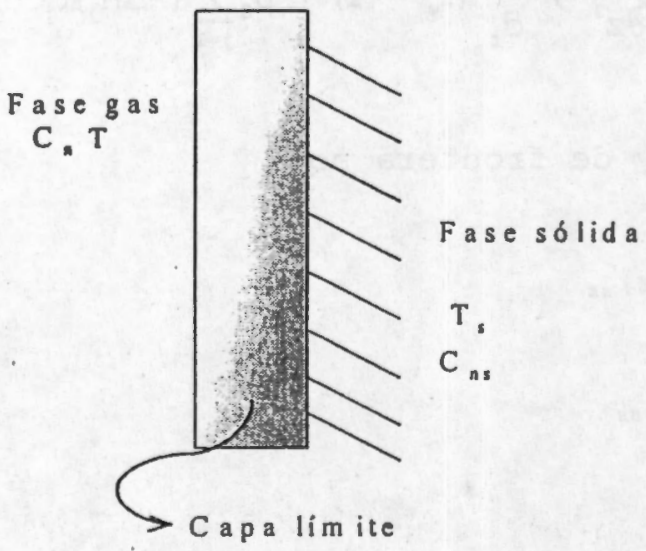

Figura 4.7 Conexión entre las fase sólido y gas. 
Capitulo 4.

Estudio del comportamiento en estado estacionario del reactor de oxidación parcial de OX $\mathrm{OAF}$

El modelo que describe esta situación física es:

Fase gas:

$\varepsilon \frac{\partial C_{n}}{\partial t}+v_{0} \frac{\partial C_{n}}{\partial z}=\varepsilon D_{e z} \frac{\partial^{2} C_{n}}{\partial z^{2}}+(1-\varepsilon) k_{g} a_{8}\left(C_{n e}-C_{n}\right)$

$\varepsilon \rho_{\varepsilon} C_{p t} \frac{\partial T}{\partial t}+V_{o} \rho_{\varepsilon} C_{p t} \frac{\partial T}{\partial z}=k_{e z} \frac{\partial^{2} T}{\partial z^{2}}+U A_{1}(T-T)+(I-\varepsilon) h_{g} a_{e}(T-T)$

Fase sólida:

$\left(\varepsilon^{\prime}\right) \frac{\partial C_{n s}}{\partial t}=(1-\varepsilon) k_{s} a_{s}\left(C_{n}-C_{n s}\right)+\rho_{n} \sum_{j=1}^{x} v_{n j} R_{j}$

$\rho_{B} C_{p=} \frac{\partial T}{\partial t}=(1-\varepsilon) h_{g} a_{s}(T-T)+\rho_{B} \sum_{j=1}\left(-\Delta H_{j} R_{j}\right.$

En donde:

En $t=0 ; C_{n s}(0, z)=C_{n \in}(0, z) s$

$T_{s}(0, z)=T_{s}(0, z):$

Las condiciones inicial $y$ de frontera para la fase gas son las mismas que las mostradas en las ecuaciones $(4 \cdot 3-4.8)$.

\subsubsection{Modelo pseudo-homogéneo en dos dimensiones espaciales}

En los modelos en una dimensión, los fenómenos de transporte de calor y masa por dispersión en la dirección radial fueron despreciados, por lo que los perfiles radiales de temperatura $y$ concentracion son planos. Los modelos en dos dimensiones 81 consideran este transporte $y$ su efecto es importante cuando se tienen gradientes importantes de temperatura en la dirección radial. Estos modelos usan el concepto de transporte efectivo para modelar el transporte de calor y masa en la dirección radial. El modelo pseudo-homogéneo en dos dimensiones espaciales (HOM-2D) está dado por: 
$\varepsilon \frac{\partial C_{n}}{\partial t}+V_{0} \frac{\partial C_{n}}{\partial z}=\varepsilon D_{e z} \frac{\partial^{2} C_{n}}{\partial z^{2}}+\varepsilon D_{e r}\left(\frac{1}{r} \frac{\partial C_{n}}{\partial r}+\frac{\partial^{2} C_{n}}{\partial r^{2}}\right)+P_{B} \sum_{j=1} v_{n j} R_{j}$

$\rho_{B} C_{p z} \frac{\partial T}{\partial t}+V_{d} \rho_{t} C_{p t} \frac{\partial T}{\partial z}=k_{e z} \frac{\partial^{2} T}{\partial z^{2}}+k_{e r}\left(\frac{1}{r} \frac{\partial T}{\partial r}+\frac{\partial^{2} T}{\partial r^{2}}\right)+\rho_{g} \sum_{j=1}\left(-\Delta H_{y} R_{j}\right.$

Las condiciones de frontera para la dirección radial están dadas por las siguientes ecuaciones:

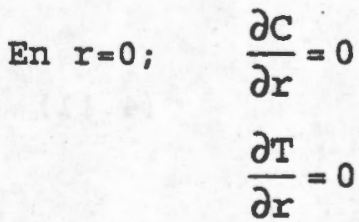

En $r=R_{e} ; \quad \frac{\partial C_{n}}{\partial r}=0$

$$
-k_{e r} \frac{\partial T}{\partial r}=h_{w}\left(T-T_{w}\right)
$$

\subsubsection{Modelo pseudo-heterogéneo en dos dimensiones espaciales}

El modelo pseudo-heterogéneo en dos dimensiones espaciales (HET-2D) incorpora la mayoría de los fenómenos que tienen lugar tanto en la fase gas como sólida en un sistema de reacción (ver la Figura 4.7). El modelo esta dado por las siguientes ecuaciones:

Fase Gas:

$\varepsilon \frac{\partial C_{n}}{\partial t}+v_{0} \frac{\partial C_{n}}{\partial z}=\varepsilon D_{e z} \frac{\partial^{2} C_{n}}{\partial z^{2}}+\varepsilon D_{e r}\left(\frac{1}{r} \frac{\partial C_{n}}{\partial r}+\frac{\partial^{2} C_{n}}{\partial x^{2}}\right)+(1-\varepsilon) k_{g} a_{s}\left(C_{n=}-C_{n}\right)$

$\varepsilon \rho_{e} C_{p t} \frac{\partial T}{\partial t}+v_{o} \rho_{f} C_{p e} \frac{\partial T}{\partial z}=k_{e z} \frac{\partial^{2} T}{\partial z^{2}}+k_{e r}\left(\frac{1}{r} \frac{\partial T}{\partial r}+\frac{\partial^{2} T}{\partial r^{2}}\right)+(1-\varepsilon) h_{g} a_{s}(T-T)$ 
Fase sరlida:

$\varepsilon \cdot \frac{\partial C_{n s}}{\partial t}=(1-\varepsilon) k_{g} a_{a}\left(C_{n}-C_{n s}\right)+p_{n} \sum_{j=1}^{x} v_{n j} R_{j}$

$\rho_{8} C_{p a} \frac{\partial T_{a}}{\partial t}=(I-\varepsilon) h_{g} a_{a}\left(T-T_{s}\right)+\rho_{z} \sum_{j=1}\left(-\Delta H_{j} R_{j}\right.$

Las condiciones inicial y de frontera están dadas por las ecuaciones (4.3-4.8), (4.13-4.14) y (4.17-4.20). Durante la predicción del comportamiento observado de este reactor, normalmente se considera flujo pistón y se emplea la velocidad superficial del sistema. Si los campos de velocidad se incorporan dentro del modelo del reactor, el término convectivo en el modelo del reactor cobra importancia, y el término de velocidad ya no es constante, sino que es una función de la posición radial y axial dentro del lecho. por lo que se debe acoplar al modelo anterior el modelo hidrodinámico para describir esta situación fisica. El modelo hidrodinámico esta dado por las siguientes ecuaciones :

La ecuación de movimiento se expresa mediante:

$$
\frac{\partial}{\partial t}\left(\rho_{\varepsilon} \varepsilon u\right)+\rho_{\varepsilon} \varepsilon u \cdot(\nabla \varepsilon u)=-\nabla \varepsilon p+\mu_{f} \nabla^{2} \varepsilon u+\rho_{t} g+R_{g-=}
$$

Las interacciones entre el solido y el gas se consideran mediante:

$$
R_{g=}=\frac{\mu_{\varepsilon}}{k(\varepsilon) d_{p_{m}}^{2}} \mathbf{u}+\frac{\rho_{f}}{k_{2}(\varepsilon) d_{p_{m}}} u \mathbf{u}
$$

$$
\begin{aligned}
& k(\varepsilon)=-\frac{1}{\alpha\left(\frac{(1-\varepsilon)^{2}}{\varepsilon^{2}}\right)} \\
& k_{2}(\varepsilon)=-\frac{1}{\beta\left(\frac{1-\varepsilon}{\varepsilon}\right)}
\end{aligned}
$$


Los perfiles de fracción vacía:

a) Cerca de la pared del lecho:

Para; $\frac{\left(R_{T}-r\right)}{d_{p}}<0.637$

$\varepsilon(r)=2.14\left(\frac{\left(R_{T}-r\right)}{d_{p}}\right)^{2}-2.53\left(\frac{\left(R_{T}-r\right)}{d_{p}}\right)+1$

b) En el centro del lecho:

$$
\begin{aligned}
& \text { Para; } \frac{\left(R_{I}-r\right)}{d_{p}}>0.637 \\
& \varepsilon(r)=\varepsilon_{\infty}+0.29 \exp \left[-0.6 *\left(\frac{\left(R_{T}-r\right)}{d_{p}}\right)\right] *\left(\cos \left(2.3 \pi\left[\frac{\left(R_{T}-r\right)}{d_{p}}-0.16\right]\right)\right. \\
& +0.15 \exp \left(-0.9\left[\frac{\left(R_{T}-r\right)}{d_{b}}\right]\right)
\end{aligned}
$$

Los detalles de estas ecuaciones se discutieron en el capitulo 2. Si se consideran los campos de velocidad en el modelamiento del reactor la condición de frontera de calor en la pared del reactor debe ser $T=T_{w}$. Sin embargo, como la componente de la velocidad radial es cero (ver capitulo 2), e igual que en el estudio de transporte de calor sin reacción, se puede emplear su aproximación de capa límite (ver capítulo 3). por lo que se utiliza la ecuación (4.20) como condición de frontera.

\subsubsection{Modelo cinético y los perfiles de desactivación}

Durante este trabajo no se realizó ningún programa experimental para evaluar cuantitativamente un esquema cinético, sino se emplea el modelo cinético de calderbank et al. [8] para estudiar diversos fenómenos en 
Capirulo 4.

Estudio del comportamiento en estado estacionario del reactor de oxidación parcial de OX a AF

el modelamiento del reactor, es decir, la hidrodinámica, el transporte de calor y la desactivación del catalizador. Este modelo cinético se basa en el modelo redox de Mars y van Krevelen [6]. El esquema de reacción consiste en seis pasos de reacción:

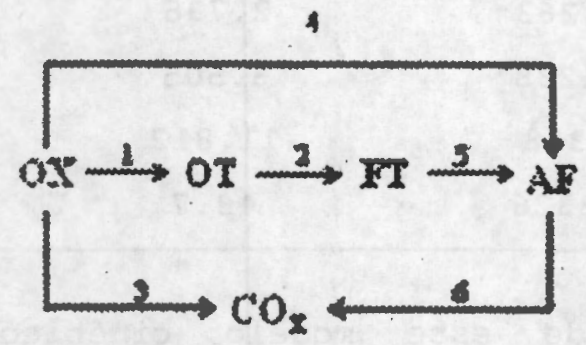

ox=o-xileno, OT=o-tolualdehido, FT=Ftalida, AF= Anhidrido Ftálico $y$ $\mathrm{CO}_{\mathrm{x}}=$ Óxidos de Carbono.

Figura 4.8. Esquema de reacción de Calderbank et al. [8].

El comportamiento de los reactantes y productos en el sistema de reacción señala que las velocidades de reacción se consideran de primer orden con respecto al hidrocarburo:

$R_{j}=\theta, k_{j} P_{n d y}$ para $j=1, \ldots, 6$

La fracción de sitios libres en el catalizador durante la evolución de la reacción esta dado por:

$\theta_{s}=\frac{k_{0} P_{o_{2} s}}{k_{0} P_{o_{20}}+\sum_{j=1}^{6} m_{j} k_{j} P_{n=j}}$

En donde:

$k_{y}=A_{j} \exp \left(-E_{a y} / R_{g} T\right)$ para $j=1, \ldots, 6$

La Tabla 4.4 da los parámetros cinéticos que se usan este trabajo. 
Tabla 4.4. Los parámetros cinéticos y calores de reacción [22]

\begin{tabular}{ccccc}
\hline$R_{j}$ & $m_{j}$ & $\begin{array}{c}-\Delta H_{j} / 10^{-3} \\
(\mathrm{~kJ} / \mathrm{kgmol})\end{array}$ & $\begin{array}{c}\mathrm{A}_{\mathrm{j}} / 10^{-3} \\
(\mathrm{kmol} / \mathrm{kg} \mathrm{h} \mathrm{htm})\end{array}$ & $\begin{array}{c}\mathrm{m}_{\mathrm{j}} / 10^{-3} \\
(\mathrm{~kJ} / \mathrm{kgmol})\end{array}$ \\
\hline 1 & 1 & 456 & 7.078 & 56.7 \\
2 & 1 & 414 & 11.45 & 56.7 \\
3 & 10.5 & 4263 & 2.736 & 56.7 \\
4 & 3 & 1238 & 6.505 & 56.7 \\
5 & 1 & 368 & 11.812 & 56.7 \\
6 & 7.5 & 3326 & 48.7 & 127.6 \\
\hline
\end{tabular}

A pesar del éxito de este modelo cintetico, diversos trabajos $[14,15,18,19,23,133,134]$ han sugerido que para tener una adecuada predicción de comportamiento observado en el reactor, tanto en estado estacionario como transitorio, se debe considerar la desactivación reversible e irreversible del catalizador. Esto se debe a que los perfiles de temperatura y concentración se desplazan como una función del tiempo de operación del reactor (ver Figuras 4.4-4.6), lo cual es imposible de predecir con un modelo cinético que no considere la desactivación del catalizador (ver Apéndice I). En el estudio del estado estacionario únicamente fue considerada la desactivación irreversible del catalizador.

Actualmente la forma más común de describir una aparente desactivación irreversibles es mediante dos formas: a) la incorporación de una expresión empirica que considera la desactivación dentro del termino de reacción en el modelo del reactor [18]; y b) la multiplicación del termino de reacción por un constante de actividad que típicamente toma valores entre 0-1 ( 6 a veces mayores), dependiendo de la zona del reactor [14,18]. En este trabajo la desactivacion irreversible se considera con un modelo empirico que considera las desactivación del catalizador por sinterizado y la fuerte adsorción de hidrocarburos a la entrada del reactor en términos de tres parámetros, los cuales están asociados con la actividad a la entrada del lecho catalítico $\left(a_{0}\right)$, la localización del punto caliente en el reactor (p) y su magnitud (q). 
Capitulo 4.

Estudio del comportamiento en estado estacionario del reactor de oridación parcial de $O X_{2} A F$

Esta ecuación esta dada por una expresión que describe una curva sigmoidal y se ajusta a partir de observaciones de temperatura en el estado estacionario [18].

$a(z)=\frac{a_{0} \exp (p z)}{1-q a_{0}[1-\exp (p z)]}$

Cuando esta expresión se incorpora a la velocidad de reaccion ésta se representa mediante:

$R_{j}=\theta_{s} k_{j} P_{n j} a(z)$ Para $j=1, \ldots, 6$

\subsubsection{Modelo adimensional del reactor}

En este punto sólo se presenta el modelo HET-2D, es decir, el de mayor complejidad en forma adimensional, ya que los demás modelos se derivan a partir de éste.

Fase gas:

$\tau_{1} \frac{\partial X_{n}}{\partial t}=\frac{1}{P_{e m z}} \frac{\partial^{2} X_{n}}{\partial \zeta^{2}}-v_{2}(\xi) \frac{\partial X_{n}}{\partial \zeta}+\frac{1}{P_{\max }}\left(\frac{\partial^{2} X_{n}}{\partial \xi^{2}}+\frac{1}{\xi} \frac{\partial X_{n}}{\partial \xi}\right)+S_{t m a}\left(X_{n=1}-X_{n}\right)$

$\tau_{1} \frac{\partial Y}{\partial t}=\frac{1}{P_{e h z}} \frac{\partial^{2} Y}{\partial \zeta^{2}}-V_{z}(\xi) \frac{\partial Y}{\partial \zeta}+\frac{1}{P_{e h r}}\left(\frac{\partial^{2} Y}{\partial \xi^{2}}+\frac{1}{\xi} \frac{\partial Y}{\partial \xi}\right)+S_{t h}\left(Y_{a}-Y\right)$

Fase s6lida:

$\tau_{1} \frac{\partial X_{n s}}{\partial t}=S_{t m}\left(X_{n}-X_{n s}\right)+\delta_{m} \hat{R}_{n}\left(X_{n=}, Y_{n}, \Omega_{0}, a(\zeta)\right)$

$\tau_{2} \frac{\partial Y_{s}}{\partial t}=S_{e n}\left(Y-Y_{s}\right)+\delta_{n} \sum_{n=1}^{N} \beta_{n} \hat{R}_{n}\left(X_{n=}, Y_{s}, \Omega_{0}, a(\zeta)\right)$ 
Con las siguientes condiciones iniciales:

AI $t=0 ; \quad(0, \xi, \zeta) ; \quad x_{n}(0, \xi, \zeta)=x_{\text {nes }}(0, \xi, \zeta)$

$$
Y_{s}(0, \xi, \zeta)=Y_{\text {nes }}(0, \xi, \zeta) ; \quad Y(0, \xi, \zeta)=Y_{e s}(0, \xi, \zeta)
$$

Y las siguientes condiciones de frontera:

Direccion axial:

En $\zeta=0 ; \quad 1=x_{n}-\frac{1}{V_{z}(\xi) P_{\text {emz }}} \frac{\partial x_{n}}{\partial \zeta}$

$$
1=Y-\frac{1}{V_{3}(\xi) P_{\text {ens }}} \frac{\partial Y}{\partial \zeta}
$$

En $\zeta=1 ; \quad \frac{\partial x_{n}}{\partial \zeta}=0$

$$
\frac{\partial Y}{\partial \zeta}=0
$$

Dirección radial:

En $\xi=0 ; \quad \frac{\partial x_{n}}{\partial \xi}=0$

$$
\frac{\partial Y}{\partial \xi}=0
$$

En $\xi=1 ; \quad \frac{\partial x_{n}}{\partial \xi}=0$

$$
-\frac{\partial Y}{\partial \xi}=B i_{w}\left(Y-Y_{w}\right)
$$

Aquí se utiliza el modelo cinético de Calderbank et al. [8] en su forma adimensional para las concentraciones en la superficie del catalizador.

$\mathbb{R}_{j}=R_{j} / R_{q}^{0}=k_{j}^{\prime} a(\zeta) \Omega_{0} Y_{*} X_{n=1}$ para $j=1, \ldots, \sigma$

$\hat{\mathfrak{R}}_{\mathrm{n}}=\sum_{j=1}^{N} \mathbb{R}_{j} \quad$ Para $\mathrm{n}=\mathrm{OX}, \mathrm{OT}, \mathrm{AF}, \ldots$, etc . 
Donde ;

$$
\begin{aligned}
& \mathbf{R}_{4}{ }^{0}=k_{d}{ }^{0} \theta_{2}{ }^{\circ} R_{g} T_{0} \bar{C}_{\alpha_{0}} \\
& k_{4}{ }^{\circ}=A_{4} \exp \left(-Y_{4}^{0}\right) \\
& k_{j}^{\prime}=\frac{k_{y}}{k_{4}^{0}}=\frac{A_{1}}{A_{4}} \exp \left(Y_{4}^{0}-Y_{9} / \bar{Y}_{4}\right)
\end{aligned}
$$

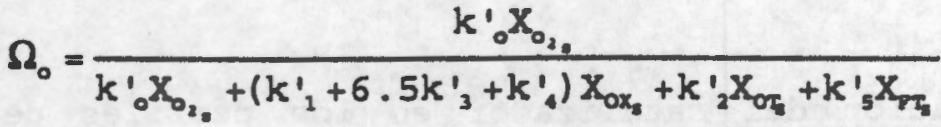

Finalmente, la expresión que considera la desactivación del catalizador en función de la posición adimensional del catalizador es:

$$
a(\zeta)=\frac{a_{0} \exp (p L \zeta)}{1-q a_{0}[1-\exp (p L \zeta)]}
$$

La Tabla 4.5 da los parámetros que se emplean en el modelo del sistema de reacción. Los parámetros de transporte de calor y masa interfaciales se obtienen de la correlaciones de Petrovic y Thodos y Aerov y Todes $[14,28,109,174]$, respectivamente. Los parámetros de transporte de calor, $U, h_{w} y k_{o r}$, se estiman de observaciones en este reactor en

\begin{tabular}{|c|c|c|}
\hline$c_{a,} \mathrm{kmol} . \mathrm{kg}_{\mathrm{atat}}{ }^{-1}$ & 0.01 & {$[10]$} \\
\hline$\varepsilon, m_{t}^{3} \cdot m^{-3} n$ & 0.48 & {$[14]$} \\
\hline$\rho_{\mathrm{f}}, \mathrm{kg} \cdot \mathrm{m}^{-3}$ & 0.54 & $\#$ \\
\hline$p_{B}, \mathrm{~kg} \cdot \mathrm{m}^{-3}$ & 1315 & {$[14,87,30]$} \\
\hline Continuación (Tabla 4.5) & & \\
\hline$D_{\text {ex }}, m^{2} \cdot h^{-1}$ & 32 & {$[28,109,174]$} \\
\hline$C_{p t}, k J(k g ~ K)^{-1}$ & 1.1 & \# \\
\hline$k_{g}, m \cdot h^{1}$ & 579 & {$[14,28,109]$} \\
\hline$h_{g}, k J \cdot m^{-2} \cdot h^{-1} \cdot K^{-1}$ & 930 & {$[7,2,3,85]$} \\
\hline
\end{tabular}
ausencia de reacción (ver capitulo 3 ). Los parámetros de dispersión de masa también se tomaron de la literatura $[28,174]$.

Tabla 4.5. Parámetros empleados en la simulación del reactor 

reactor de axidación parcial de OX a AF

$\begin{array}{lll}\text { (Continuacion Tabla } & 4.5) \\ k_{\text {er }} \mathrm{kJ} \cdot \mathrm{m}^{-1} \cdot \mathrm{h}^{-1} \cdot \mathrm{K}^{-1} & 8.2 & {[27] \text { * }} \\ \mathrm{h}_{\mathrm{w}} \mathrm{kJ} \cdot \mathrm{m}^{2} \cdot \mathrm{h}^{-1} \cdot \mathrm{K}^{-1} & 457 & {[27] \text { * }} \\ \mathrm{U}, \mathrm{kJ} \cdot \mathrm{m}^{2} \cdot \mathrm{h}^{-1} \cdot \mathrm{K}^{-1} & 532 & {[27] \text { * }}\end{array}$

- Ese 1 mado de los experimentos atr reacción cuando se incorpora la hidrodinámica y se cansidera no-isotropia en el aiatema de rescción

* Calculados a la temperatura de reacción

\subsection{Efecto de la desactivación del catalizador en los perfiles de temperatura y concentración}

En esta sección se estudia la sensibilidad del perfil de actividad en la respuesta del modelo del reactor. Está se divide en dos puntos. En el primero estudia la sensibilidad de los perfiles de temperatura y concentración ante variaciones en el perfil de actividad que se establece en la superficie del catalizador en el estado estacionario. Esto se realiza al manipular los parámetros $p$ y $q$ de la Ecuación desactivación del catalizador (Ecuación (4.28)). En el segundo se predicen los datos experimentales a las condiciones de operación del Exp-1 y Exp-3, empleando la familia de modelos del reactor mostrados en la sección 4.6 .

\subsubsection{Efecto de los parámetros de la Ecuación de desactivación del catalizador}

Las Figuras 4.9 y 4.10 muestran la sensibilidad de los perfiles de temperatura y concentración ante variaciones en el perfil de actividad que se establece en la superficie del catalizador en el reactor. Esto se realiza manipulando los parámetros de ajuste $a_{0}, p$ y $q$ de la Ecuación (4.28). Aquí sólo se muestra el efecto de $p$ y q que manipulan el punto de la inflexión y el máximo de la sigmoide y están unidos de tal forma que se asocian con la magnitud y posición del punto caliente, afectando directamente a la cinética de la reacción. La simulación del 
Capirulo 4.

Estudio del comportamiento en estado estacionario del reactor de oxidación parcial de OX a AF

reactor se realiza utilizando las condiciones de operación del Exp-1 y el modelo ном-2D.

La Figura 4.9 a muestra el efecto de $q$ en los perfiles de temperatura y concentración mientras $a_{0}$ y q permanecen constantes. El valor de $q$ fija la magnitud del punto caliente. La variación de este parámetro de ajuste provoca un efecto en los perfiles de temperatura similar al que se observó al hacer un estudio de sensibilidad de los parạmetros de transporte de calor, $h_{w} y k_{\text {er }}$. Esto se puede observar en la referencias [87,89] Y en el Apéndice J. Sị el catalizador sufre desactivación por sinterizado mínima, como ha sido reportado $[15,19], y$ todas las posibles gomas formadas de hidrocarburo adsorbidas en el catalizador han sido oxidadas, el valor de $q$ debe ser 1 . De otra forma se tendra un efecto importante e indeseable en la cinética de las reacciones, $y$ esto no es recomendable.

La Figura 4.10a muestra la sensibilidad de los perfiles de concentración y temperatura al variar $p$ de la Ecuación (4.28) y que se asocia con la posición del punto caliente en el reactor. Este parámetro no modifica la magnitud del punto caliente, por lo que su variación se relaciona con la desactivación del catalizador a la entrada del lecho.

Los perfiles de actividad, que se tienen al variar $q$ y en la ecuación (4.28), se muestran en las Figuras 4.9b y 4.10b, respectivamente. En donde la forma del perfil de actividad se relaciona con los perfiles de temperatura y concentración en el reactor. Esto muestra que la sigmoide es una expresión que no tiene más justificación que hacer que la predicción del modelo ajuste los datos experimentales, sin embargo, su uso es necesario. 

reactor de coxidación parcial de OX a AF
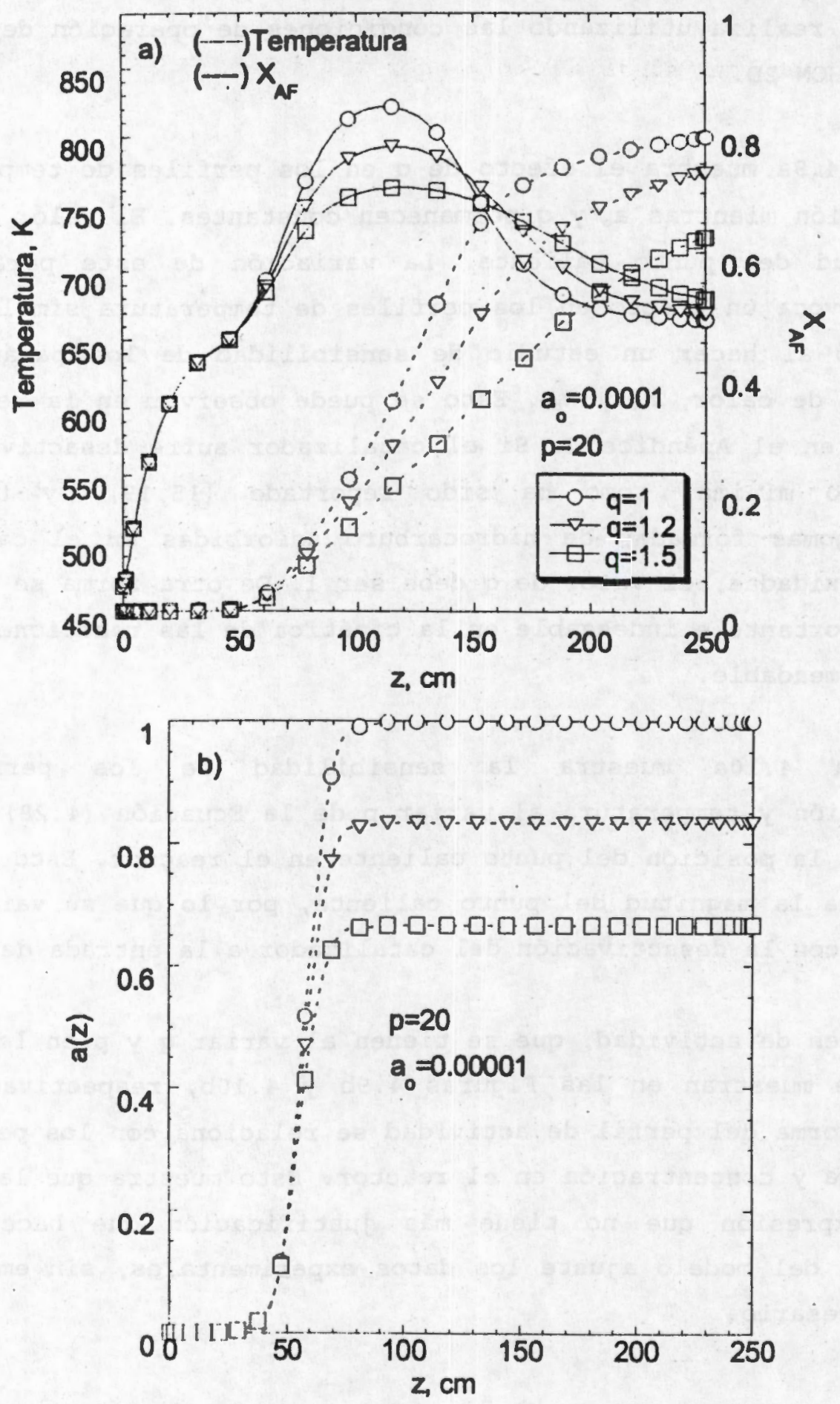

Figura 4.9. Efecto del parámetro q de la función de desactivación. (a) En los perfiles de concentración y temperatura y (b) en el valor de la función de desactivación $a(z)$, en función de la posición axial del reactor. 

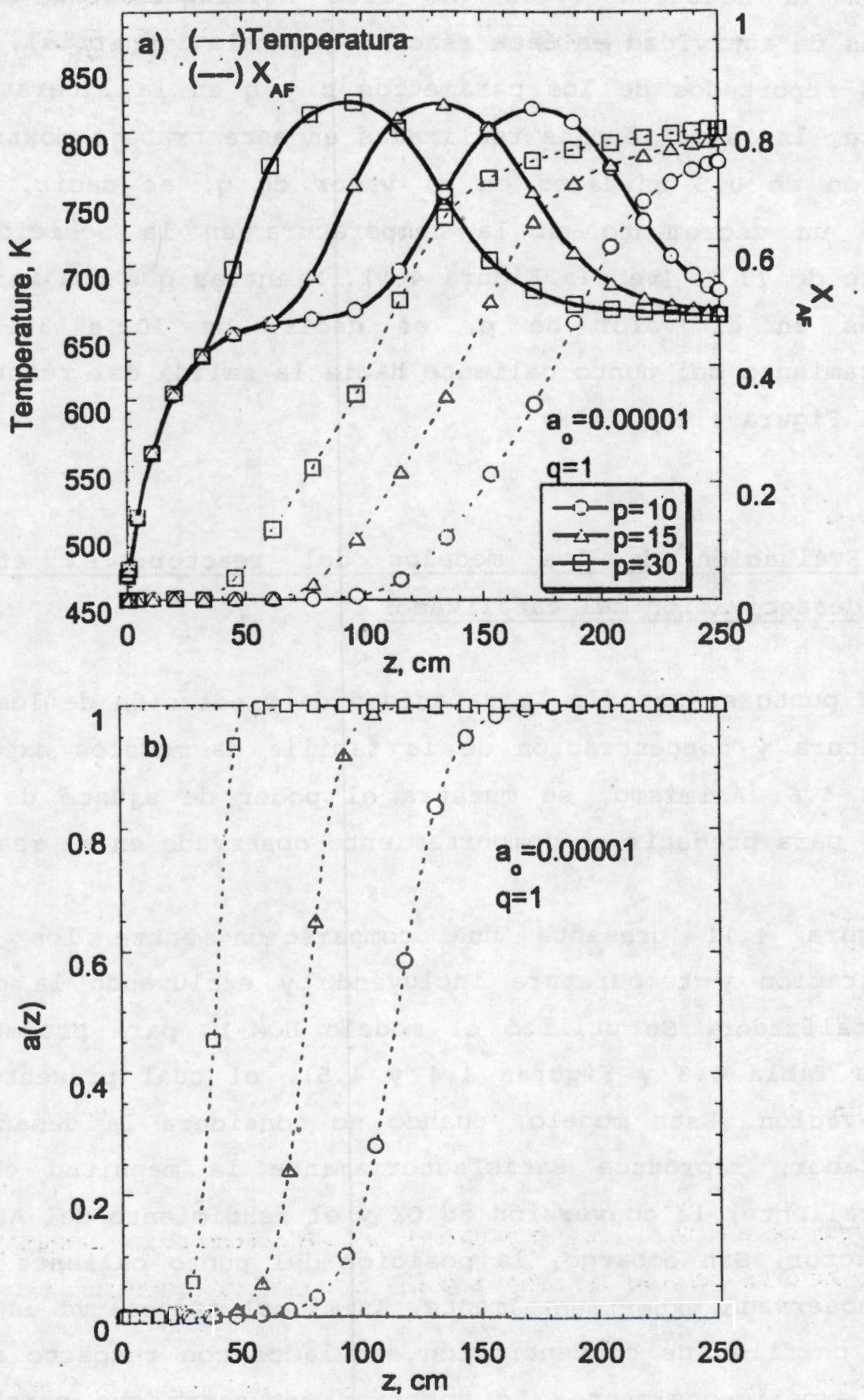

Figura 4.10. Efecto del parámetro p de la función de desactivación. (a) En los perfiles de concentración y temperatura y (b) en el valor de la función de desactivación a(z), en función de la posición axial del reactor. 
Si bien la Ecuación (4.28) ha sido utilizada para describir los perfiles de actividad en éste reactor a escala industrial, no se tienen valores reportados de los parámetros $p$ y $q$ en la literatura [14]. No obstante, las simulaciones realizadas en este trabajo mostraron que una variación de 0.5 unidades en el valor de $q$, es decir, de 1 a 1.5 , provocó un decremento en la temperatura en la posición del punto caliente de $70^{\circ} \mathrm{C}$ (ver la Figura 4.9). Mientras que una variación de 20 unidades en el valor de $p$, es decir, de 10 a 30 , provoco un desplazamiento del punto caliente hacia la salida del reactor de $100 \mathrm{~cm}$ (ver la Figura 4.10).

\subsubsection{Evaluación de los modelos del reactor: el efecto de la} desactivación del catalizador

En este punto se estudia la capacidad de predicción de los perfiles de temperatura y concentración de la familia de modelos expuestos en la sección 4.6. Asimismo, se muestra el poder de ajuste de la Ecuación (4.28), para predecir el comportamiento observado en el reactor.

La Figura 4.11 presenta una comparación entre los perfiles de concentración $y$ temperatura incluyendo $y$ excluyendo la desactivación del catalizador. Se utilizó el modelo HoM-1D para predecir el Exp-1 (ver la Tabla 4.3 y Figuras 4.4 y 4.5), el cual presenta una fuerte desactivación. Este modelo, cuando no considera la desactivación del catalizador, reproduce satisfactoriamente la magnitud observada del punto caliente, la conversión de ox y el rendimiento del AF a la salida del reactor. Sin embargo, la posición del punto caliente está alejada de la observada experimentalmente. Asimismo, se observa un defasamiento de los perfiles de concentración simulados con respecto a los que se tienen experimentalmente. Lo anterior demuestra que para predecir de manera adecuada el comportamiento observado de temperatura $y$ concentración, se tiene que emplear una función que tome en cuenta a la desactivación del catalizador existente a la entrada del lecho. Cuando se usa la Ecuación (4.28) para considerar la desactivación del 
Capitulo 4.

Estudio del comportamiento en estado estacionario del reactor de oxidación parcial de OX a AF

catalizador se observa un comportamiento igual al experimental, ya que los perfiles de concentración y temperatura se desplazan hacia la salida del reactor, lo cual indica que a la entrada del reactor la velocidad de reacción es muy baja, casi nula.

En esta Figura también se observa que la amplitud en el perfil de temperatura predicho es mayor que el observado, lo cual ha sido reportado ya en la literatura $[14,15]$. Esto podría deberse a que la desactivación que sufre el catalizador en la zona alrededor del punto caliente no ha sido considerada adecuadamente en el modelo cinético [14,18] (ver el capitulo 5). Esta desactivación podría relacionarse con la adsorción de hidrocarburos y su efecto en la dinámica oxidoreducción que ocurre en la superficie activa del catalizador.

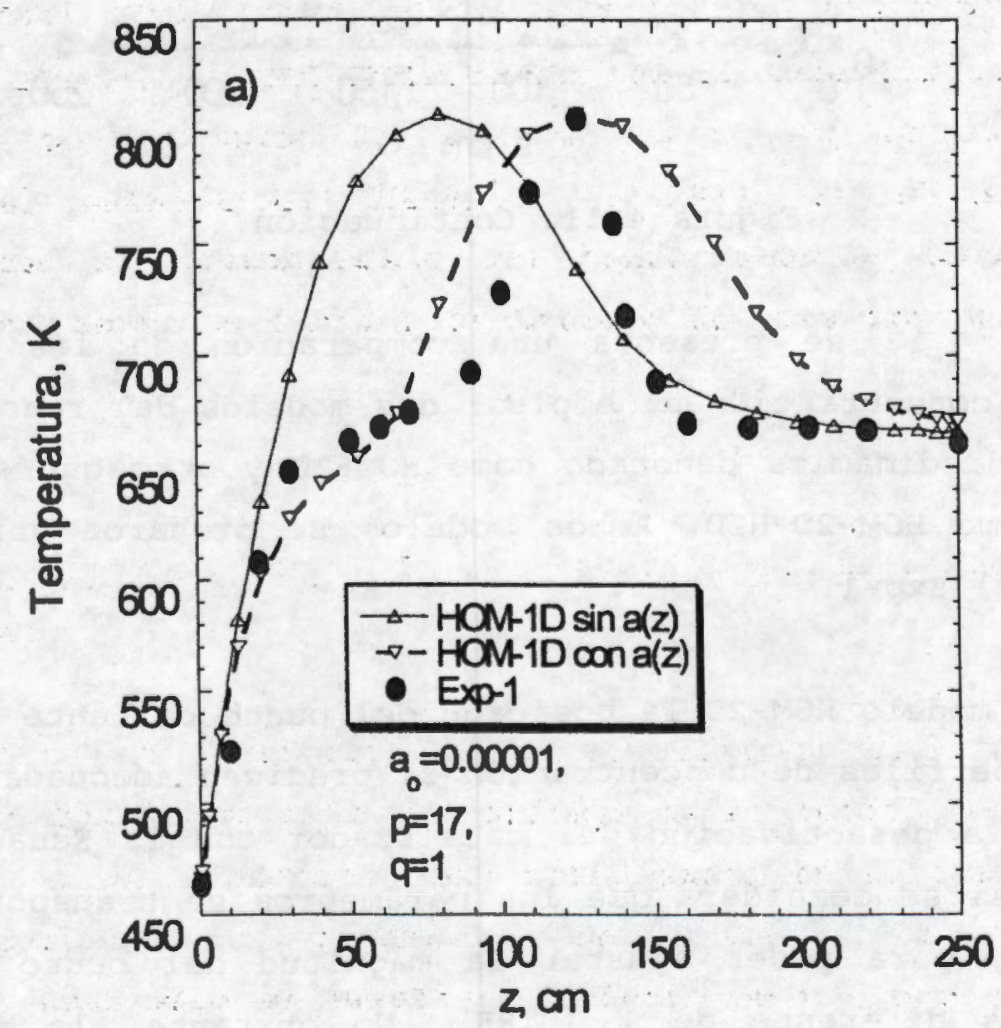

Figura 4.11. Efecto de la desactivación del catalizador en la predicción del modelo HOM-1D a las condiciones del Exp-1. (a) Los perfiles de temperatura; (b) los perfiles de concentración. 


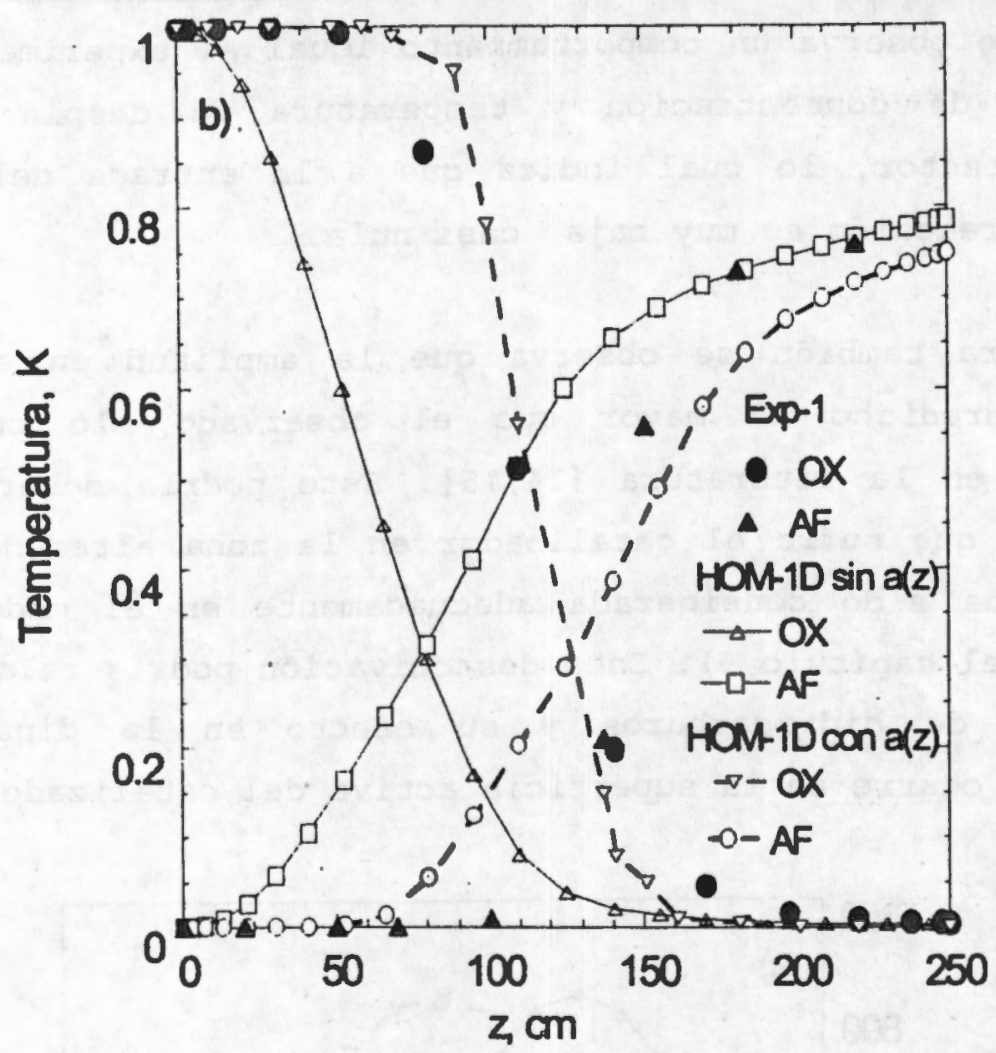

Figura 4.11. Continuación.

En la Figura 4.12 se presenta una comparación de los perfiles de temperatura y concentración al emplear dos modelos del reactor: uno que excluye la hidrodinámica denotado como HOM-2D y otro que si lo hace $y$ se refiere como HOM-2D-HID. Ambos modelos se probaron utilizando las condiciones del Exp-1.

En el uso del modelo HOM-2D la posición del punto caliente experimental así como los perfiles de concentración se predicen adecuadamente cuando se consideró la desactivación del catalizador con la Ecuación (4.28). Sin embargo, si se considera que los parámetros de transporte de calor son correctos, para poder ajustar la magnitud del punto caliente el valor de $q$ es diferente de 1 (0.85). No obstante, la amplitud del perfil de temperatura axial sigue estando alejada de la experimental. 

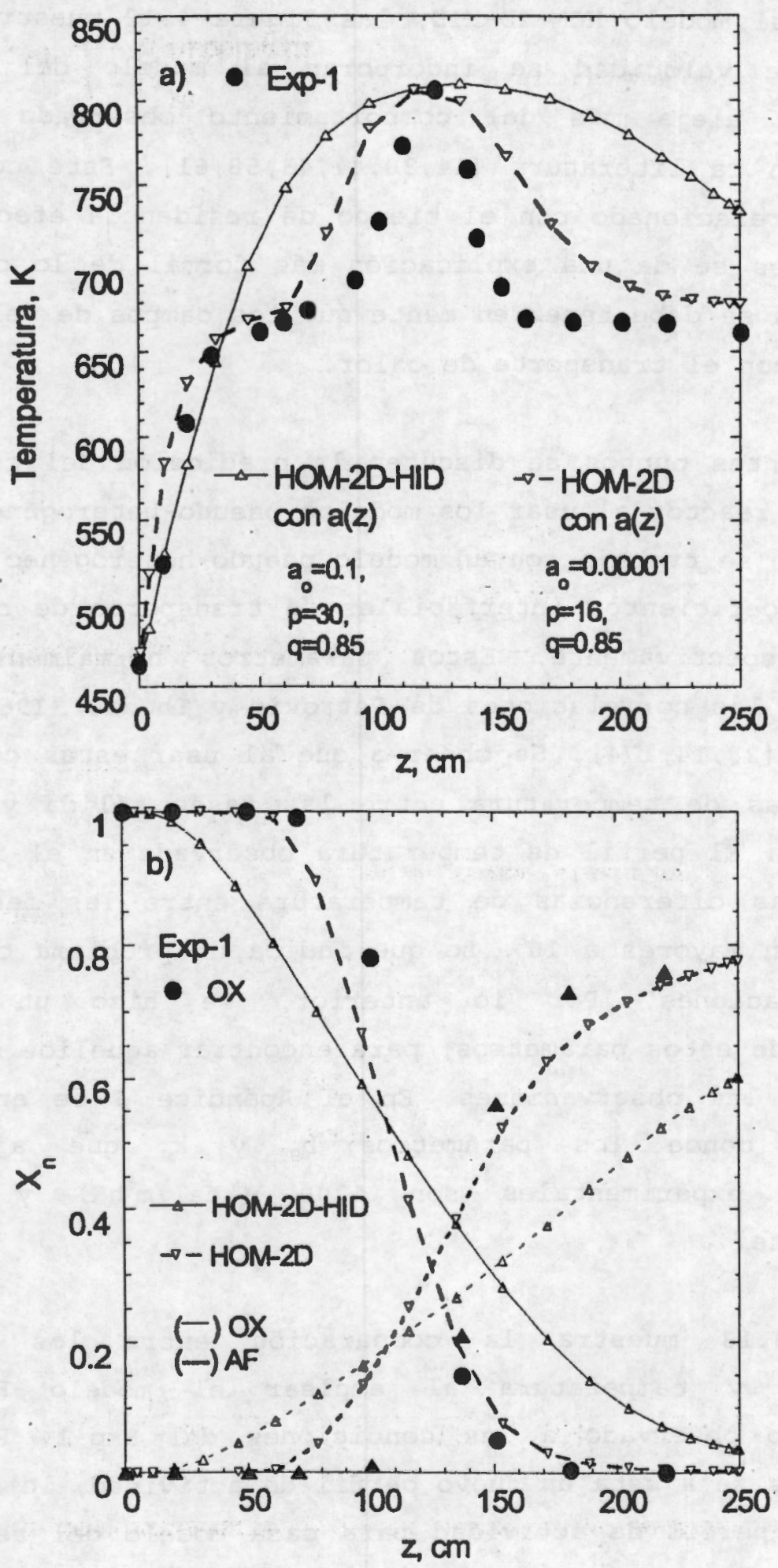

Figura 4.12. Evaluación de los modelos HOM-2D y HOM-2D-HID en la predicción del Exp-1. a) Temperatura y b) Concentración. 
Con respecto al modelo HOM-2D-HID, las Figura 4.12 muestra que cuando los campos de velocidad se incorporan al modelo del reactor, la predicción se aleja más del comportamiento observado lo cual se contradice con la literatura $[14,30,41,43,58,61]$. Este comportamiento podría estar relacionado con el tiempo de residencia efectivo. En los próximos puntos se da una explicación más formal de lo observado. En estos momentos se debe tener en mente que los campos de velocidad están relacionados con el transporte de calor.

En los siguientes puntos se discuten la predicción del comportamiento observado del reactor al usar los modelos pseudo-heterogéneos, HET-ID Y HET-2D. Cuando se trabaja con un modelo pseudo-heterogéneo es necesario conocer los coeficientes interfaciales de transporte de calor y masa, $h_{g} y k_{g}$. respectivamente. Estos parametros normalmente han sido evaluados con las correlaciones de Petrovic y Thodos (1968) y Aerov y Todes (1968) $[28,14,174]$. Se observo que al usar estas correlaciones, las diferencias de temperatura entre las fases sólida y gaseosa son mayores a $20^{\circ}$. El perfil de temperatura observado en el reactor [14], mostro que las diferencias de temperatura entre las fases solida y gaseosa no son mayores a $10^{\circ}$. Lo que indica un problema con el uso de estas correlaciones. Por 10 anterior, se hizo un estudio de sensibilidad de estos parámetros, para encontrar aquellos que describan adecuadamente las observaciones. En el Apéndice $J$ se encuentra este estudio, en donde los parámetros $h_{g} y \quad k_{g}$ que ajustaron las observaciones experimentales son 1200 (kcal/ $\left.\mathrm{m}^{2} \mathrm{hK}\right)$ y 1000 (m/h) respectivamente.

La Figura 4.13 muestra la comparacion entre los perfiles de concentración $y$ temperatura al emplear el modelo HET-1D $y$ el comportamiento observado a las condiciones del Exp-1. Para predecir estos perfiles se ajusta un nuevo perfil de actividad; lo que indica el ajuste de un perfil de actividad para cada modelo del reactor que se utilice. Al usar el modelo HET-1D tampoco se puede predecir la amplitud experimental del perfil de temperatura. 

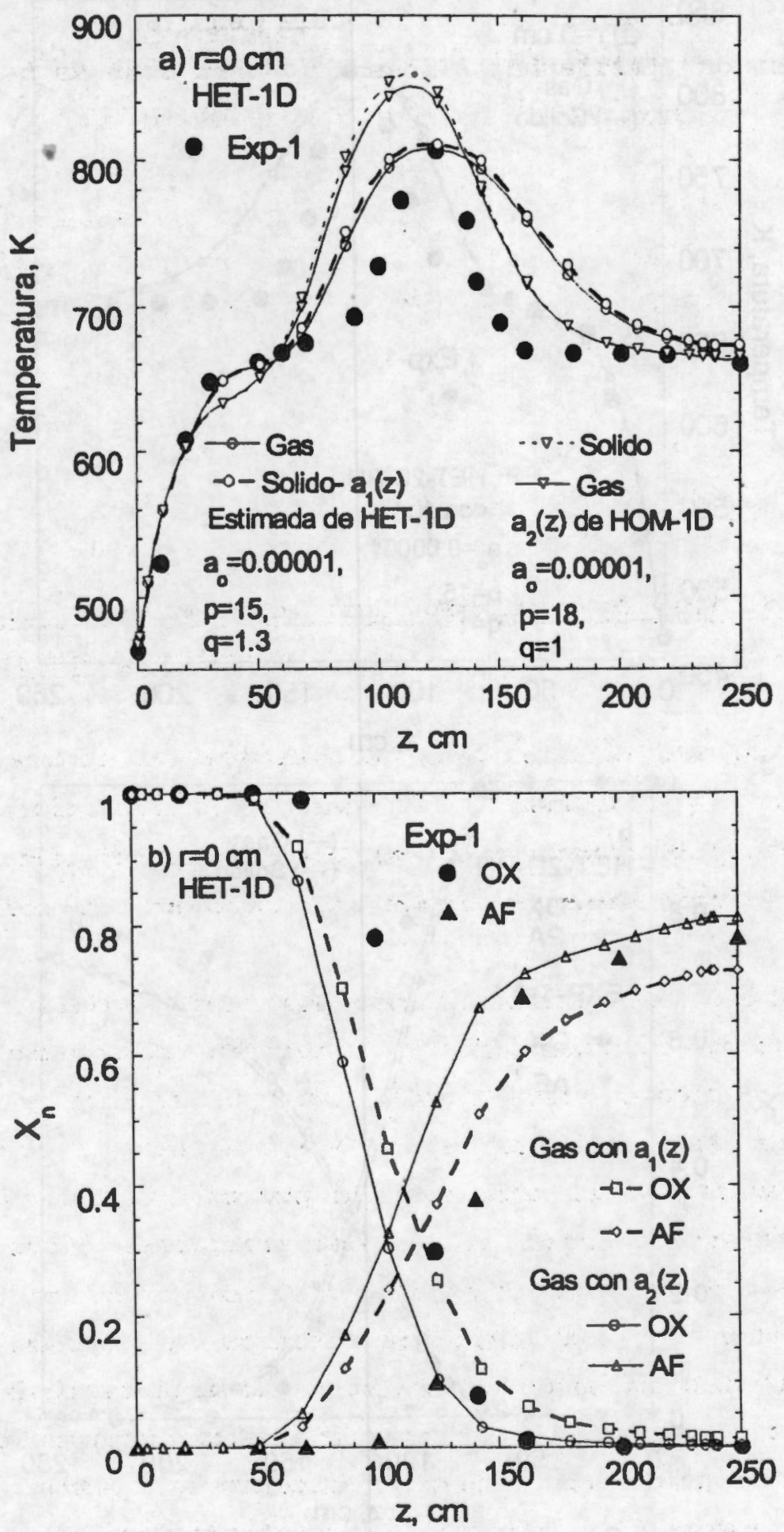

Figura 4.13. Predicción del Exp-1 con el modelo HET-ID considerando dos diferentes perfiles de desactivación. a) Temperatura y b) concentración. 

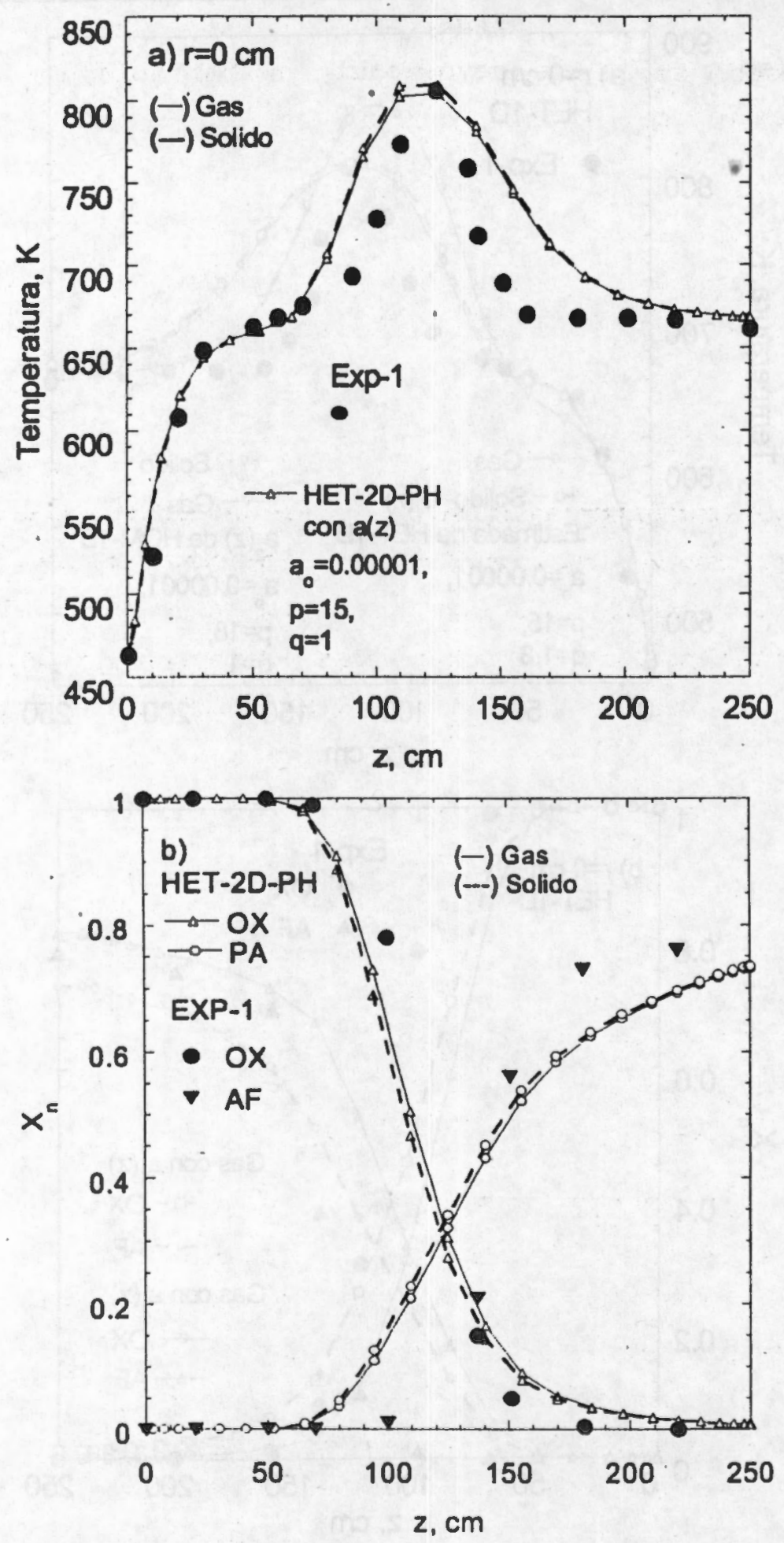

Figura 4.14. Predicción de los perfiles de temperatura y concentración del EXP-I empleando el modelo HET-2D-PH. a) Temperatura y b) concentración. 


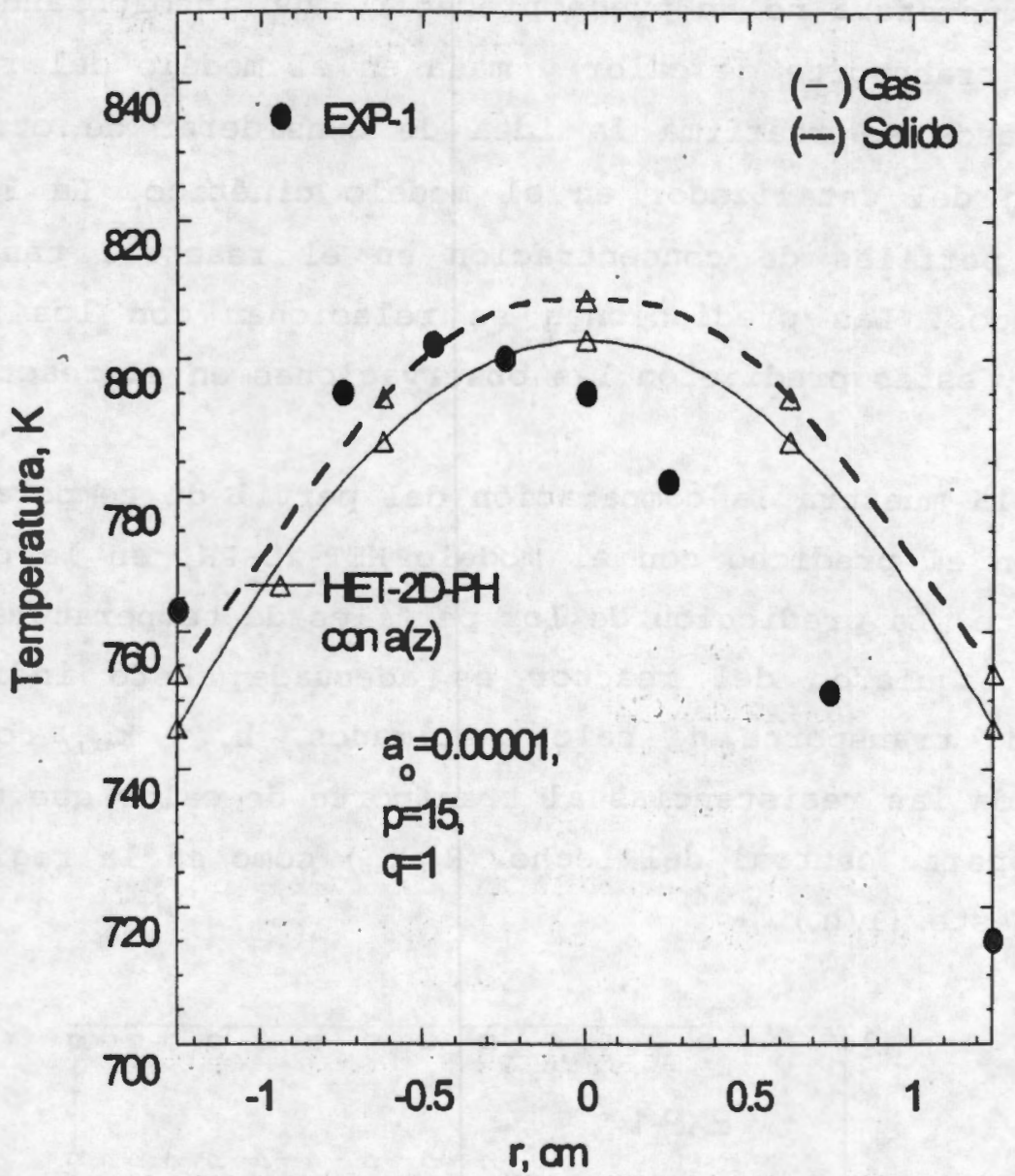

Figura 4.15. Predicción del perfil de temperatura radial del Exp-1 con el modelo HET-2D-PH.

Las Figuras 4.14 y 4.15 muestran una comparación entre los datos experimentales de temperatura y concentración del Exp-1; con aquellos predichos al utilizar el modelo HET-2D. Para la simulación del Exp-1 se emplean los parámetros de transporte de calor estimados considerando la aproximación de capa límite y no-1sotropía en el lecho (ver el capitulo 3). Este modelo es nombrado HET-2D-PH.

En la Figura 4.14 a se presentan los perfiles axiales de temperatura. Las predicciones muestran que para predecir los resultados experimentales es necesario ajustar un perfil de actividad diferente al que se ajusto con otro modelo del reactor. La magnitud y posición del punto caliente es similar a lo observado, sin embargo, la amplitud del 

reactor de oxidación parcial de OX a AF

perfil de temperatura no se puede predecir, aún incorporando todos los fenómenos de transporte de calor y masa en el modelo del reactor. Con estos resultados se reafirma la idea de considerar de otra forma la desactivación del catalizador en el modelo cinético. La Figura 4.15b muestra los perfiles de concentración en el reactor: tanto predicho como observados. Las predicciones se relacionan con los perfiles de temperatura y estas predijeron las observaciones en el reactor.

La Figura 4.15 muestra la comparación del perfil de temperatura radial observado con el predicho con el modelo HET-2D-PH, en la posición del punto caliente. La predicción de los perfiles de temperatura observados en el lado izquierdo del reactor es adecuada. Esto indica que los parámetros de transporte de calor estimados, $h_{1}, y k_{a x}$, consideran de forma adecuada las resistencias al transporte de calor que tienen lugar tanto en la parte central del lecho $\left(R_{t} / k_{e r}\right)$ como en la region cerca de la pared de éste $\left(1 / h_{w}\right)$.

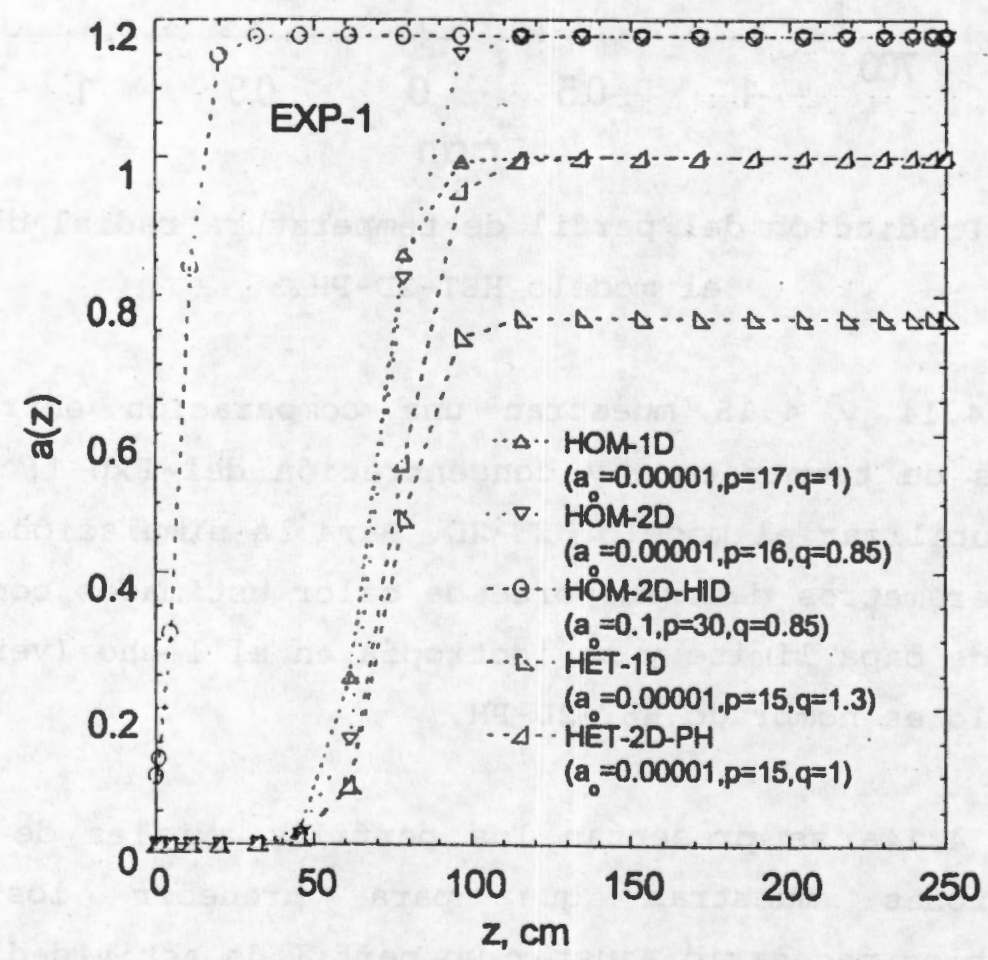

Figura 4.16. Comparacion de los perfiles de actividad émpleando diferentes modelos del reactor para predecir el Exp-1. 

reactor de cxidación parcial de OX a AF

La Figura 4.16 muestra una comparación de los perfiles de actividad que se tendrían en el lecho, si se considera que todos los modelos del reactor son factibles para predecir su comportamiento. Un criterio para elegir el modelo adecuado del reactor se relaciona con el ajuste del parámetro q de la Ecuación (4.28). En este caso q debe ser igual a uno, lo que implica que sea considerado lo siguiente: que los parametros de transporte de calor, $h, y k_{\text {or }}$, son correctos, que el catalizador sufre una desactivación por deformaciones estructurales casi nula y el modelo cinético predice adecuadamente la magnitud del punto caliente sin incorporar la ecuación $(4.28),(14,15,18,19,23,133,134)$. Si se considera que en este reactor se tienen gradientes radiales de temperatura entre 5 y $40^{\circ}$, dependiendo de su posición axial. (ver la Eigura 4.4), el modelo HET-2D-PH es necesario para su simulación, además que este modelo incorpora a los fenómenos que son indispensables, tanto de transporte de calor como de masa.

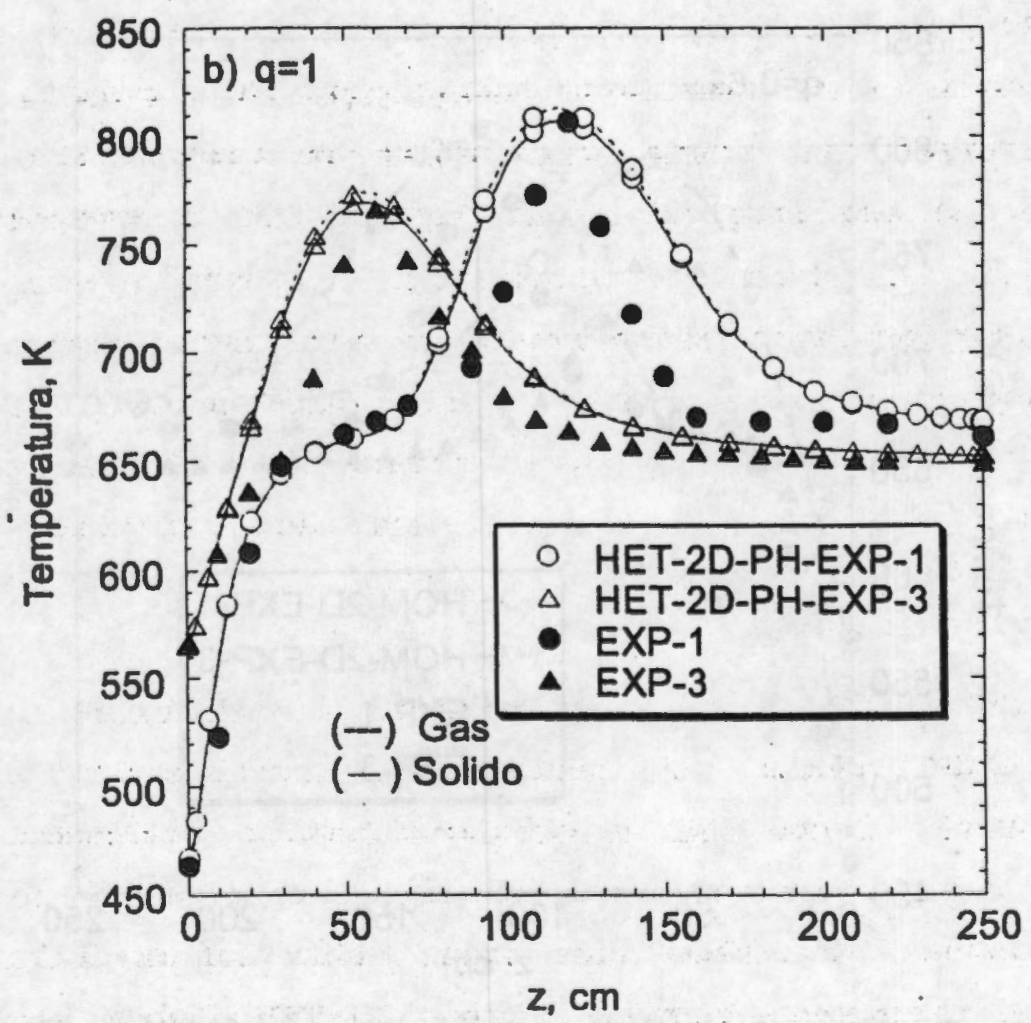

Figura 4.17. Predicción del modelo HET-2D-PH al emplear diferentes condiciones de operacion. 

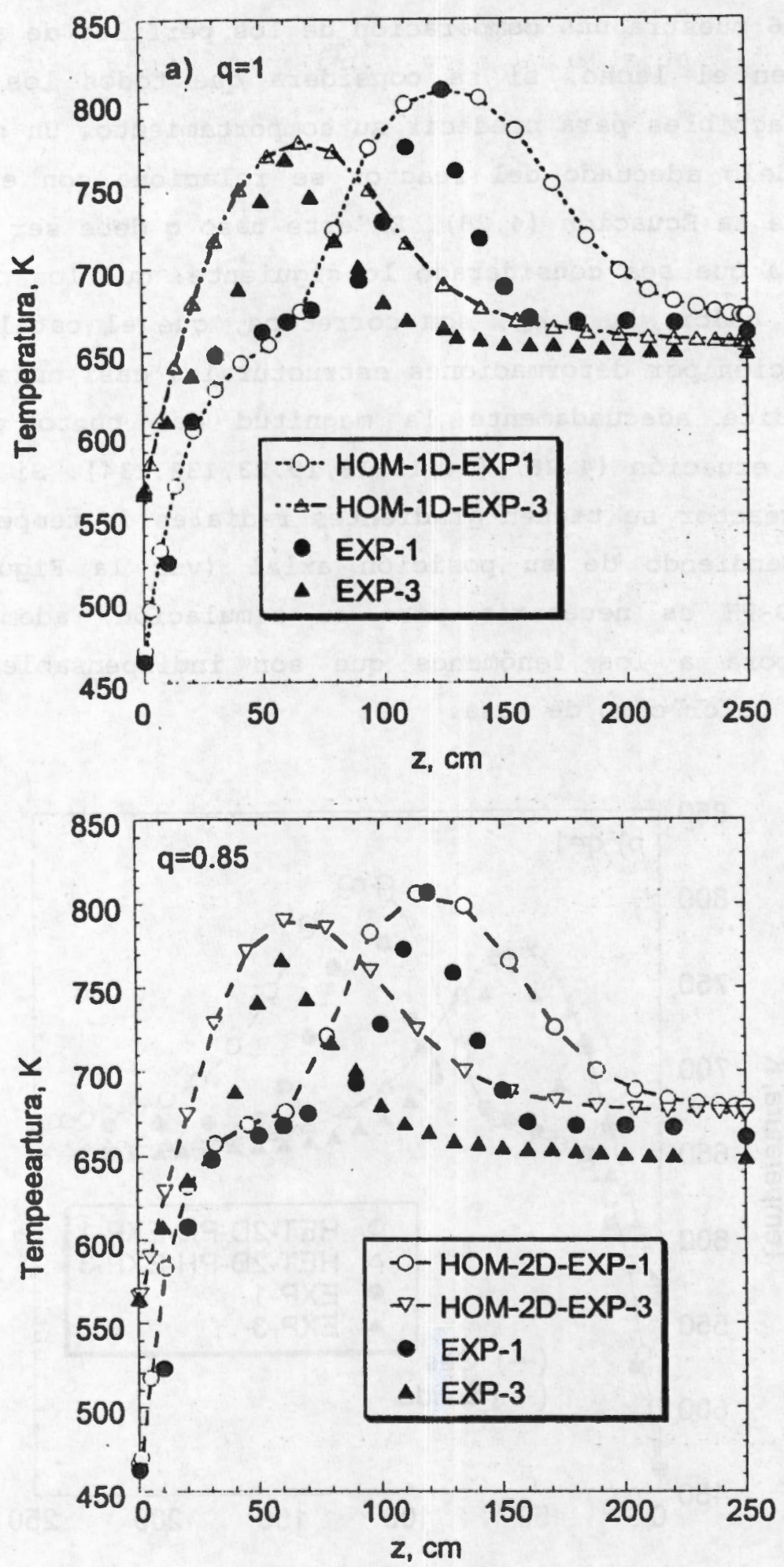

Figura 4.18. Predicción de los modelos homogèneos a dos diferentes condiciones de operación. a) HOM-1D y b) HOM-2D. 

reactor de coxidación parcial de $\mathrm{OX} 2 \mathrm{AF}$

Los resultados que se presentan en las Figuras 4.17 y 4.18 , muestran dos aspectos importantes: La eficiencia en la predicción de las observaciones a diferentes condiciones de operacion (EXP-3), utilizando el modelo HET-2D-PH; que los modelos homogéneos, HOM-1D Y HOM-2D, 10 cuales pueden ser utilizados para hacer estudios exploratorios del reactor en estado estacionario, teniendo como principal ventaja, el no necesitar de los coeficientes interfaciales de transporte de masa y calor, y que los tiempos computo son más cortos.

\subsection{Simulación del reactor al emplear los parámetros de transporte de calor estimados sin reacción.}

En esta sección se discuten la predicción del modelo HET-2D al utilizar los parámetros de transporte de calor, $h_{w} y k_{e r}$, estimados en ausencia de reacción en el reactor. Estos parámetros de transporte de calor se estiman de dos maneras: (1) de la forma clásica, que es cuando se considera flujo pistón en el lecho; (2) al considerar el transporte de momento al de calor: la forma más apropiada cuando se tiene un sistema con baja relación de $d_{t} / d_{p}$. Estas dos formas de estimar los parámetros de transporte de calor se realizaron bajo dos situaciones: al considerar isotropia en el lecho y al considerar no-isotropía en el (vex también Capítulo 3 ).

El objetivo de esta sección es usar estos parámetros de transporte de calor para predecir el comportamiento observado en el reactor. Esta sección se divide en dos partes: primero, se compara la predicción del modelo al utilizar $h_{w} y k_{e r}$ estimados tanto de la forma clásica como considerando la hidrodinámica; después, se discute la predicción del modelo al utilizar $h_{w} y k_{a r}$ que se estimaron al considerar isotropía, $y$ no isotropla en el lecho. 
4.8.1 Sensibilidad de $h_{v} y k_{e r}$ estimados de dos maneras: de la forma clásica y considerando la hidrodinámica

Las Figuras 4.19 y 4.20 muestran dos comparaciones entre los perfiles de temperatura y concentración, observados de los experimentos I y 3 , respectivamente, con aquellos predichos al usar el modelo HET-2D. Este modelo se evalúa bajo dos casos: a) al emplear 108 parámetros de transporte de calor estimados de la forma clásica, HET-2D-PC; b) al emplear los parámetros de trasporte de calor estimados al considerar la aproximación de capa limite, HET-2D-PH. La estimación de $h_{w} y k_{a x}$, no consideran isotropia en el lecho.

Debido a la sensibilidad que tiene la simulación del reactor a los parámetros de transporte de calor (ver también el Apéndice J). es necesario realizar la estimación independiente de éstos. Sin embargo, el estimarlos del mismo sistema de reacción, no garantiza tener los parámetros adecuados. Esto se observa al comparar las predicciones de los perfiles de temperatura y concentractón al usar el modelo HET-2D-PH $y$ el HET-2D-PC, en donde las diferencias de temperatura y concentracion son importantes. Estos resultados muestran la importancia del estudio hidrodinámico y de transporte de calor desarrollados en los capítulos 2 y 3 , en los cuales se obtienen aportaciones novedosas que son importantes en el estudio con reacción del sistema de estudio.

Se encontro que el modelo HET-2D-PH predice mejor los perfiles de temperatura y concentración de los experimentos 1 y 3 , que el modelo HET-2D-PC. Sin embargo, se debe buscar una explicación que pueda fundamentar los resultados obtenidos. Asimismo, se observa que la menor temperatura en la posición del punto caliente se obtuvo cuando se emplea el modelo HET-2D-PH, esto se debe a que los valores de $h_{w} y k_{e x}$ son mayores que los usados en el modelo HET-2D-PC (ver la Tabla 3.3), teniendo menores resistencias al transporte de calor en la parte central del lecho $\left(R_{t} / k_{a r}\right)$ y en la región cercana a la pared $\left(1 / h_{w}\right)$. teniendo como consecuencia un mejor enfriamiento. 

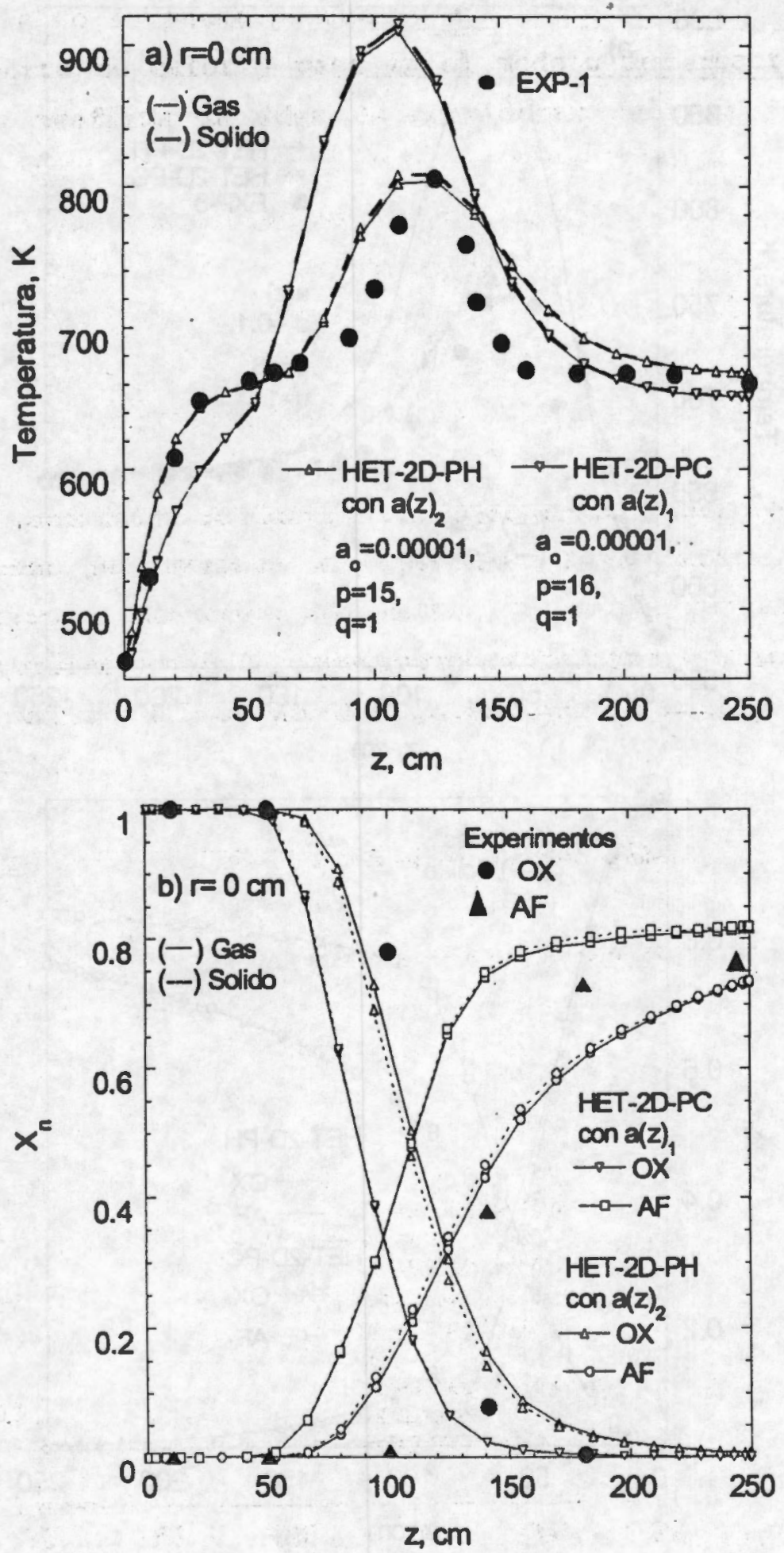

Figura 4.19. Predicción del modelo al usar los parámetros de transporte de calor estimados de la forma clásica y con la hidrodinámica, considerando no-isotropía en el reactor; el Exp-1; el modelo HET-2D. 

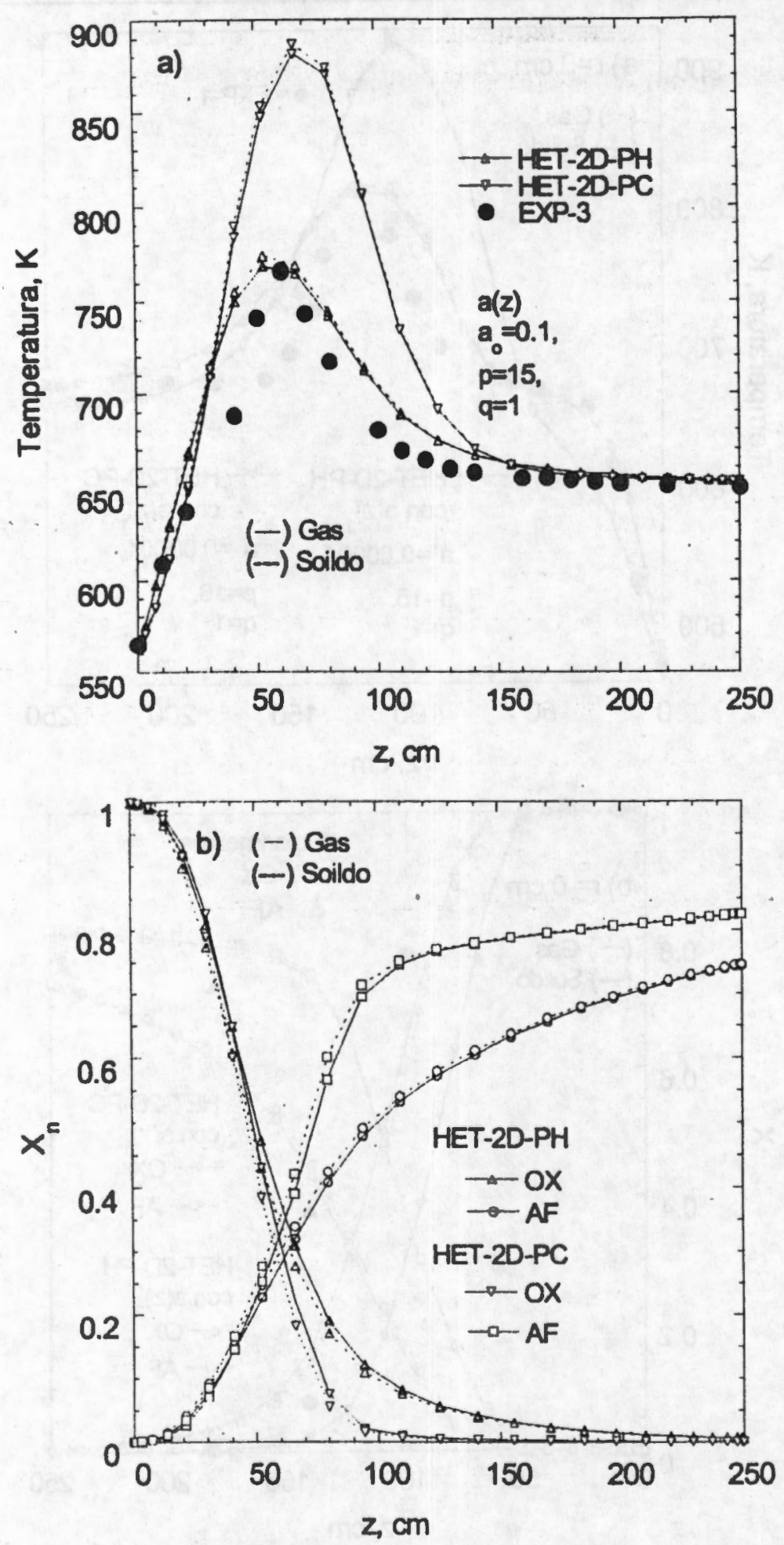

Figura 4.20. Predicción del modelo al usar los parámetros de transporte de calor estimados de la forma clásica y con hidrodinámica, considerando no-isotropía en el reactor; el Exp-3; el modelo HET-2D. 

reactor de oxidación parcial de $\mathrm{OX}$ a AF

La simulación del reactor permitio observar dos cosas: el incorporar el modelo hidrodinámico al modelo del reactor no da buena aproximación la predicción de las observaciones (ver la Figura 4.12), y el usar el modelo HET-2D-PH predice, con excepción de la amplitud del perfil axial de temperatura, el comportamiento observado.

La revistón del estado del arte sobre un problema que no ha podido ser esclarecido sugiere que el éxito de la predicción adecuada del comportamiento observado al usar el modelo HET2D-PH, se debe a que los parámetros de transporte de calor, al ser estimados como un promedio a lo largo del radio, contienen en forma intrínseca tanto los procesos de transporte de calor estáticos como los dinámicos $[27,38]$. Por esto, no es necesario incorporar el modelo hidrodinámico al modelo del reactor. Por esto, se sugiere que si se quiere considerar el transporte de momento en el modelamiento del reactor, los parámetros de transporte de calor deben incorporar explicitamente dos términos, uno que contenga los procesos de transporte de calor estáticos y otro los dinámicos $[18,19,23,31-33,133,134]$. En donde el término dinámico se verá afectado por la velocidad del fluido.

\subsubsection{Simulación del reactor utilizando los parámetros de transporte de} calor estimados considerando isotropía en el lecho

Una de las principales aportaciones en el estudio del transporte de calor (ver el capítulo 3 ), es su estudio utilizando dos tipos de experimentos en ausencia de reacción, recurriendo a un lecho de laboratorio $(40 \mathrm{~cm})$, operado en formas casi-adiabatica y no-adiabática. En ambos experimentos se considera isotropia, lo que facilita encontrar de forma más sencilla los mínimos globales. Estos experimentos en conjunto con el modelo de transporte de calor en el lecho, son utilizados para estimar los parámetros correspondientes. Esto permitio estimar estos parámetros sin usar ninguna correlación de la literatura. 
En la Figura 4.21 se presenta una comparación de los perfiles de temperatura al utilizar los parámetros de transporte de calor estimados bajo la consideración de isotropia en el lecho. Estos parámetros se estiman bajo dos situaciones: (1) utilizando un modelo clásíco que no incluye la hidrodinámica en el lecho empacado; (2) incorporando la hidrodinámica. Los resultados confirman que un buen ajuste de las observaciones en el reactor es posible cuando se incorporan los campos de velocidad y se considera Isotropía 6 no Isotropía en la estimación de los parámetros de transporte de calor en ausencia de reacción. .

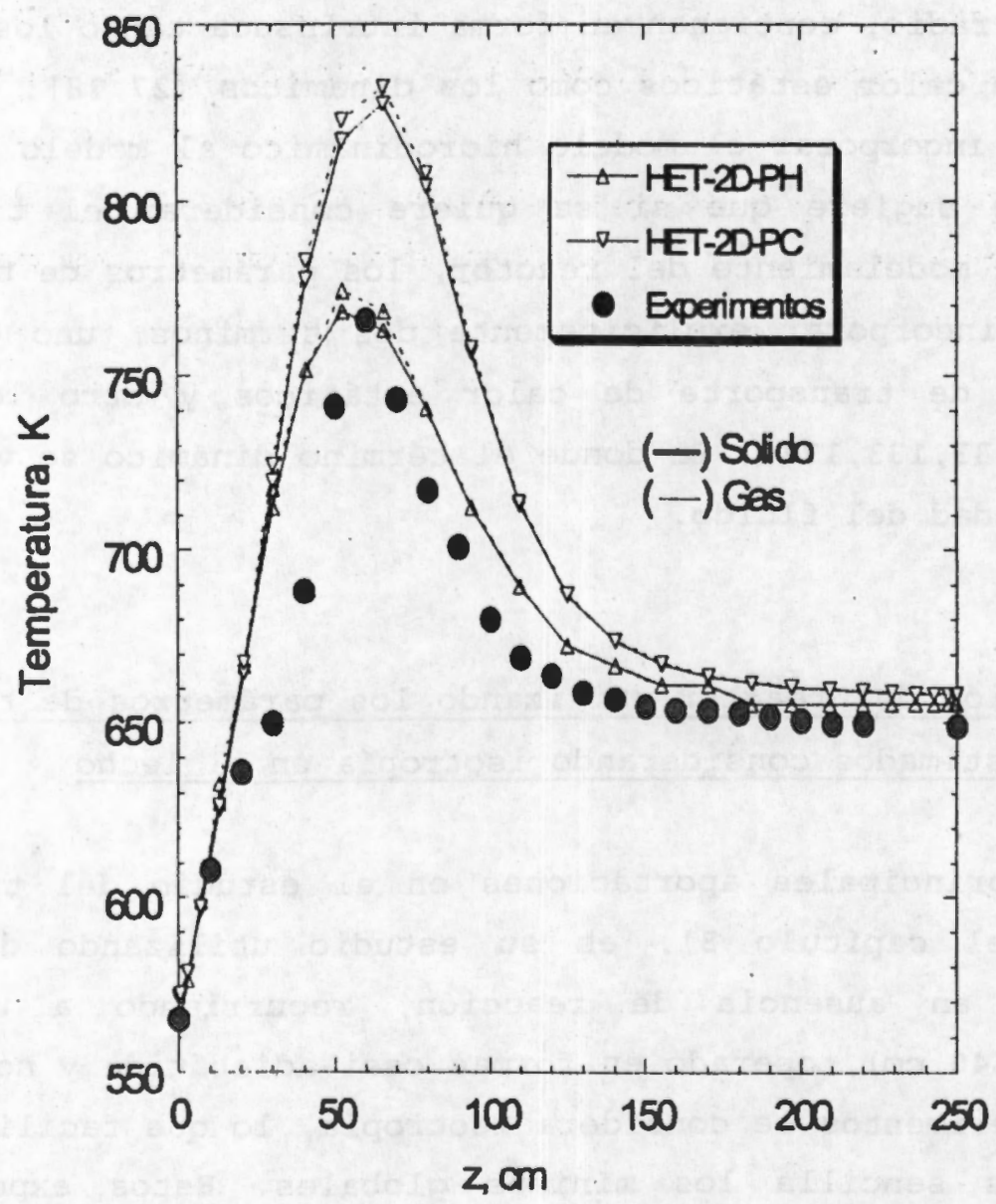

Figura 4.21. Predicción del modelo al usar los parámetros de transporte de calor estimados de la forma clásica y con hidrodinámica, considerando isotropía en el reactor; el Exp-3; el modelo HET-2D. 
Capitulo 4.

Estudio del cömportatniento en estado estacionario del reactor de oxidación parcial de $\mathrm{OX}$ a $\mathrm{AF}$

Uno de los puntos que queda pendiente en este trabajo, es comparar la predicción de los perfiles de temperatura y concentración, cuando se emplean los parámetros de transporte de calor estimados considerando los campos de velocidad en el lecho, y utilizando la condición de frontera en el radio del tubo, $T=T_{w}(z)$, metodología con la cual la $k_{a r}$ es el único parámetro a estimar. Lo anterior se debe a que en los experimentos que se tienen, no se conoce la temperatura en la pared del tubo a lo largo de todo el lecho.

\subsection{Conclusiones y perspectivas}

En el presente capitulo se desarrollo un estudio del estado estacionario de la reacción de oxidación parcial de ox a AF en un reactor a escala piloto. Se emplean modelos del reactor con diferentes complejidades; el modelo cinético de calderbank et al. para describir el coraportamiento del catalizador de V/Ti; y una ecuación empírica para considerar la desactivación del catalizador. Ia comparación de la simulación del reactor con su comportamiento observado mostró lo siguiente:

a) Con respecto a los parámetros de transporte de calor

1) El modelamiento del sistema de reacción es muy sensible a pequeños cambios en los parámetros de transporte de calor: $k_{e z}$, $k_{\text {er }} y h_{w}$. Por esto, es necesario un estudio independiente de transporte de calor en el lecho de estudio. El considerar isotropía y no isotropía junto con el transporte de momento en el lecho, durante la estimación de $k_{\text {er }} y h_{w}$ en ausencia de reacción, es una alternativa para predecir, el comportamiento observado en el reactor.

2) Cuando se incluyo la hidrodinámica en la simulación del reactor, las predicciones están alejadas con respecto al comportamiento observado. Sin embargo, al considerar flujo pistón en el 
reactor, se predice el comportamiento observado si se utilizan los parámetros de transporte de calor estimados, en ausencia de reacción, al incluir la hidrodinamica. El modelo. HET-2D-PH predice esta situación. Estos resultados sugieren que dichos parametros incluyen intrinsicamente los procesos de transporte de calor estáticos y los dinámicos que ocurren en el reactor.

3) Para esta clase de reactores, que operan con reacciones altamente exotérmicas, es indispensable estimar los coeficientes interfaciales de transporte de calor $y$ masa. Ya que la comparación de la predicciones del modelo del reactor con observaciones en éste, muestran que las correlaciones que cuantifican estos parámetros [14,28,174], sobre-estiman la temperatura experimental del catalizador.

b) Con respecto al catalizador:

2) El comportamiento experimental del reactor muestra la presencia de dos fenómenos de desactivación en la superficie del catalizador: una reversible $y$ otra irreversible. Esta desactivación se considera con una expresión empirica que describe a una sigmoide (ver la Ecuación 4.28), la cual contiene tres parámetros de ajuste, $a_{0}$, $p$ q. Los cuales se ajustan a partir de observaciones de temperatura en el reactor. El valor de p modifica la magnitud del punto caliente; ao y $p$ la desactivación reversible e irreversible a la entrada del lecho.

4) El valor de $q$ debe ser igual a uno, si se considera que los parámetros de transporte de calor y el modelo cinético son los correctos. Lo que se sugiere utilizar el valor de $q$ como un criterio para elegir el modelo del reactor. Para el estudio del reactor en estado estacionario, el modelo pseudo-heterogéneo es necesario para la simulación de esta clase de reactores. Sin embargo, los modelos pseudo-homogéneos son de gran utilidad para realizar estudios exploratorios de algunas variables que ayuden 

reactor de cridación parcial de $\mathrm{OX}$ a AF

a entender su comportamiento, además que los tiempos de computo son mucho menores:

En estudios en estado estacionario, el incluir la desactivación del catalizador con una ecuación empirica, que describe a una curva sigmoide, parece ser una forma exitosa para predecir la magnitud y posición del punto caliente y los perfiles de concentración. Sin embargo, la no adecuada predicción de la amplitud del perfil de temperatura axial, sugiere que, en próximos estudios, los procesos de desactivación se deben incluir dentro del modelo cinético, como se propone en el Apéndice $I$, en donde se da un esquema cinético que se basa en dos puntos: i) Describir la adsorción de las diversas moléculas en la superficie del catalizador y su parcial oxidación a $\mathrm{CO}_{x} ;$ y il) incorporar la dinámica oxido-reducción en la superficie del sólido. Lo que probablemente permitirá la predicción del estudio de esta clase de reactores en estado transitorio (ver capítulo 5). 


\title{
Capítulo 5.
}

Una introducción al comportamiento transitorio del reactor de oxidación parcial de oxxileno a

\author{
anhídrido ftálico
}



de oxidación parcial de oxileno a anhidrido frilice

5. Dna Introducción al conportantento traneltorlo del reactor de oxidación parcial de o-xileno a anhidrldo Ftrilico

En la operación de cierta clase de reactores de lecho fijo en forma transitoria se ha observado la existencia de múltiples estados estacionarios $[8-11,15,16,18,19,28,31,32,33,36,175-181]$. En la oxidación parcial de o-xileno (Ox) a anhidrido Ftalico (AF), utilizando un catalizador de $\mathrm{V}_{2} \mathrm{O}_{5} / \mathrm{TiO}_{2}$ (V/Ti), esta multiplicidad ha sido relacionada con la desactivación reversible e irreversible del catalizador $[15,18,19,11]$. Actualmente, no existe un modelo cinético capaz de predecir el comportamiento transitorio observado en el reactor.

En este capitulo se toma como referencia el mecanismo propuesto por Calderbank et al. (1977) [8], el estudio en estado transitorio del reactor de oxidación de OX a AF desarrollado por Lopez-Isunza et al. (1992) [14] Y las ideas expuestas en el Apéndice $\check{~}$ sobre el catalizador, con $e$ : fin de explicar, teoricamente, el comportamiento transitorio del reactor de oxidación parcial de ox a AF.

Este capitulo se divide en cuatro secciones:

1. En la sección 5.1 se describen los antecedes sobre el comportamiento transitorio del reactor de oxidación parcial de ox a AF.

2. En la sección 5.2 se plantea un modelo cinético, que considera la desactivación reversible del catalizador. Asi mismo, este modelo es validado, al ajustar el comportamiento observado en el reactor en el estado estacionario.

3. En la sección 5.3 se utiliza este modelo cinético para predecir cualitativamente el comportamiento transitorio del reactor, al incrementar la temperatura del baño en forma de rampa. 

de oxidación parcial de oxileno a anhidrido fralice

4. En la sección 5.4 se presentan las conclusiones y perspectivas sobre el estudio transitorio del reactor.

\subsection{Antecedentes}

Varios son los trabajos $[8-11,15,16,18,19,28,31,32,33,36,175-181]$ que se han interesado en estudiar la multiplicidad de soluciones en el reactor de lecho fijo para reacciones altamente exotérmicas. Esta multiplicidad de soluciones ha sido observada tebrica $[10,28,31$ $33,36,130,177,179]$ y experimentalmente $[9,15,16,16,19,144,175,176,180]$, al estudiar la sensibilidad de los perfiles de temperatura $y$ concentración al variar las condiciones de operación del reactor.

En la oxidación parcial de $O X$ a $A F$ en un reactor de lecho fijo a escala industrial, se han observado experimentalmente múltiples estados estacionarios al variar reversiblemente la temperatura del baño de enfriamiento $(14,15,16,18,19,144]$. Algunos trabajos sugieren que la causa principal de esta multiplicidad se relaciona con la desactivación reversible que sufre el catalizador de $\mathrm{V}_{2} \mathrm{O}_{5} / \mathrm{THO}_{2}(\mathrm{~V} / \mathrm{Ti})$ durante la reacción $[18,19,11]$.

López-Isunza et al. $[14,18]$ presenta un estudio té6rico-experimental en estado transitorio de un reactor industrial a escala de banco, para la reacción de OX a AF. Éste muestra el desplazamiento del perfil axial de temperatura al cambiar la temperatura del baño reversiblemente, en donde se obtienen dos perfiles axiales de temperatura diferentes a la misma temperatura del baño (ver la Figura 5.1). Estos resultados fueron discutidos como sigue: 


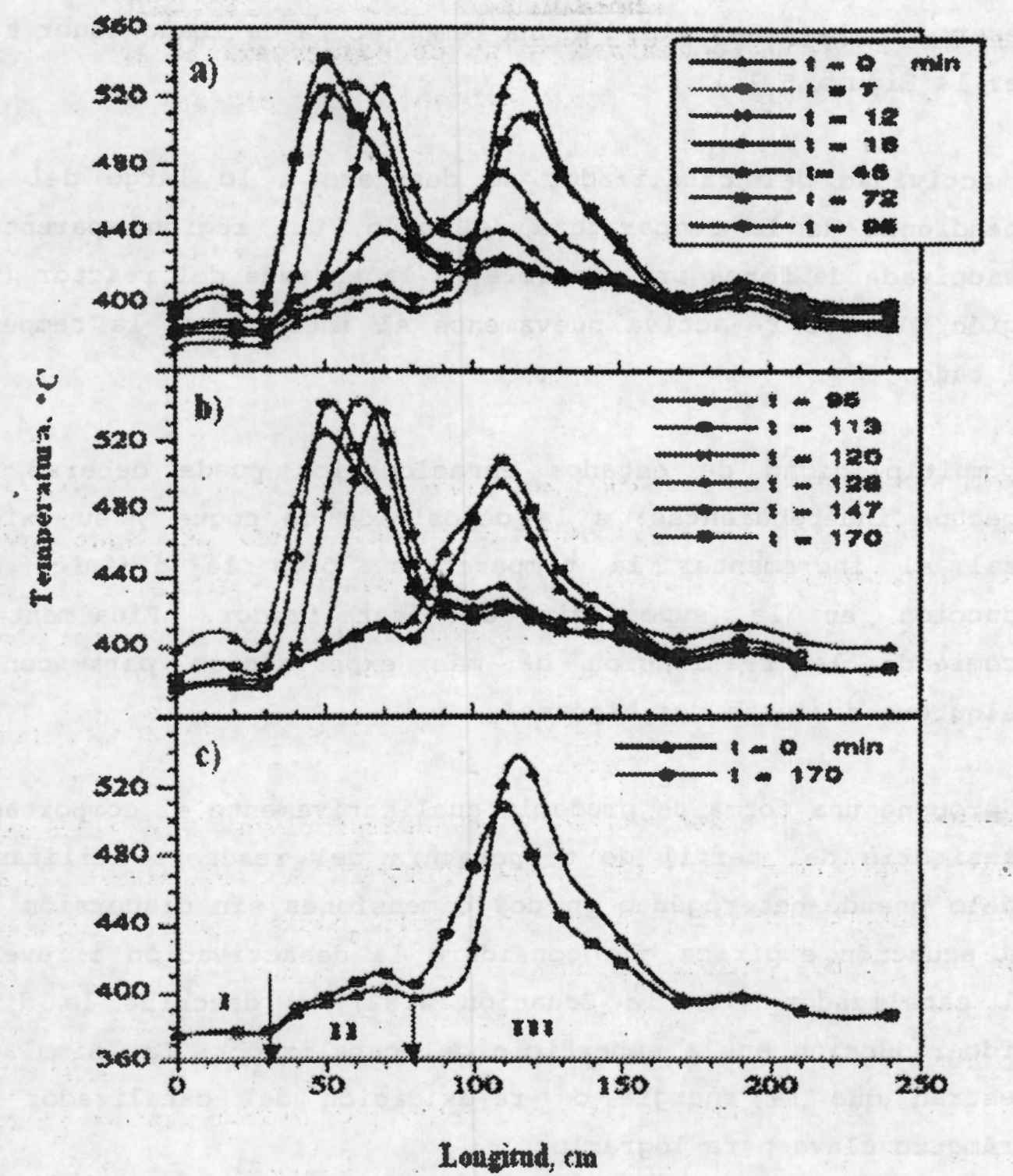

Figura 5.1. Desplazamiento de los perfiles de temperatura a) al variar la $\mathrm{T}_{\mathrm{b}}$ de $390{ }^{\circ} \mathrm{C}$ a $408^{\circ} \mathrm{C}$, b) al variar la $\mathrm{T}_{\mathrm{b}}$ de $408^{\circ} \mathrm{C}$ a $390^{\circ} \mathrm{C}$ y c) $\mathrm{la}$ comparación del estado estacionario inicial y final a la misma $T_{b}$ (390 $\left.{ }^{\circ} \mathrm{C}\right)[18]$.

a) En la región. I (los primeros $30 \mathrm{~cm}$ ), el catalizador aparenta mostrar una desactivación irreversible; en la región II (los siguientes $50 \mathrm{~cm}$ ), el catalizador se desactiva de forma reversible mostrando una alta actividad a una temperatura del baño mayor a 673 
$\mathrm{K} ; \mathrm{Y}$ en la región III (en los ultimos $160 \mathrm{~cm}$ ), el catalizador muestra una baja actividad a una temperatura del baño menor a $668 \mathrm{~K}$ (ver la Figura 5.1c).

b) La actividad del catalizador se desplazo a lo largo del lecho, dependiendo de la temperatura del baño. Una región aparentemente desactivada de forma irreversible, a la entrada del reactor (ver la región II), se re-activa nuevamente al incrementar la temperatura del baño.

c) La multiplicidad de estados estacionarios puede deberse a dos aspectos independientes: a la deposición de coque y su oxidación total al incrementar la temperatura, 6 a la dinámica oxidoreducción en la superficie del catalizador. Finalmente, se recomienda la realización de más experimentos para confirmar cualquiera de estas dos hipótesis.

d) Se propone una forma de predecir cualitativamente el comportamiento transitorio del perfil de temperatura del reactor: utilizando un modelo pseudo-heterogéneo en dos dimensiones sin dispersión axial; una ecuación empirica que considera la desactivación irreversible del catalizador (ver la Ecuación 4.82); $y$ describe la dinámica oxido-reducción en la superficie del catalizador. Las simulaciones muestran que la energía de re-oxidación del catalizador es un parámetro clave para lograrlo.

Actualmente este estudio $[16,18]$, es la mejor aproximación que se tiene para predecir cualitativamente el comportamiento transitorio de la reacción de OX a AF. Sin embargo, este muestra algunas inconsistencias: diversos estudios señalan que el catalizador sufre una desactivación irreversible insignificante $[15,19]$; la oxidación del catalizador es muy rápida, y se sugiere que la desactivación del catalizador se debe a la fuerte adsorción de moléculas de $O X$, las cuales se polimerizan en la superficie del catalizador $[15,19,11]$; además se sugiere que el vanadio, durante la reacción, presenta dos estados de oxidación $\left(V^{+5}\right)$ y 

de oxidación parcial de oxileno an anhidrido furlico

$\left(\mathrm{v}^{+3}\right)$, los cuales son los responsables de la oxidación parcial y total del hidrocarburo, respectivamente [11]; el modelo cinético no es validado; al considerar la desactivación reversible del catalizador no se predice la amplitud del perfil axial de temperatura.

Mongkhonsi et al. [19], realiza un experimento similar para la misma reacción y reactor (ver la Figura 5.2). Sin embargo, al comparar los perfiles de temperatura de estos trabajos se observan algunas diferenclass. Mongkhonsi et al. [15,19] obtiene una mayor temperatura en la posición del punto caliente en el ultimo estado estacionario, mientras que López-Isunza et. al. $\{14,18\rceil$ la obtiene en el primero. Esto muestra la complejidad del estudio en estado transitorio de este reactor.

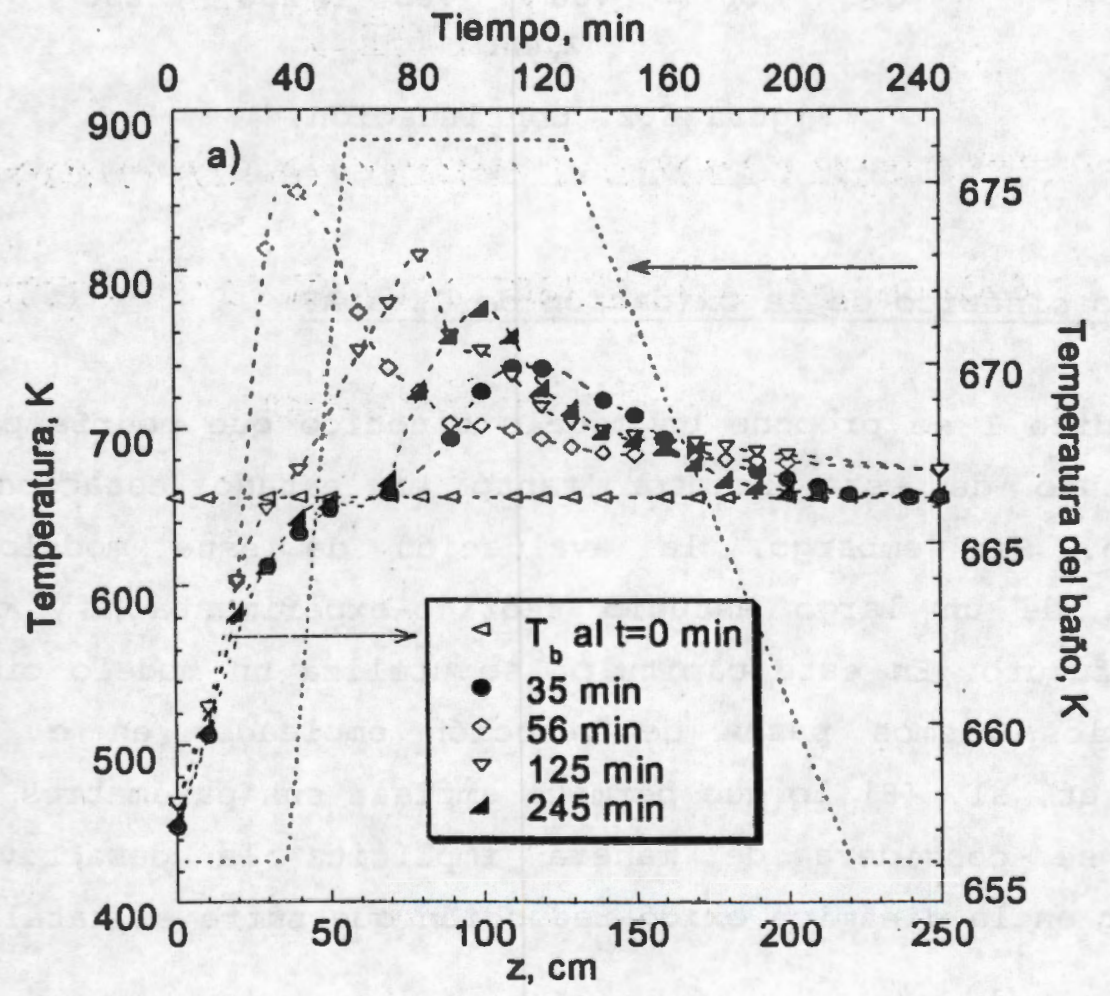

Figura 5.2. Comportamiento de la temperatura en el interior del reactor a cambios reversibles de la temperatura del baño [19] 


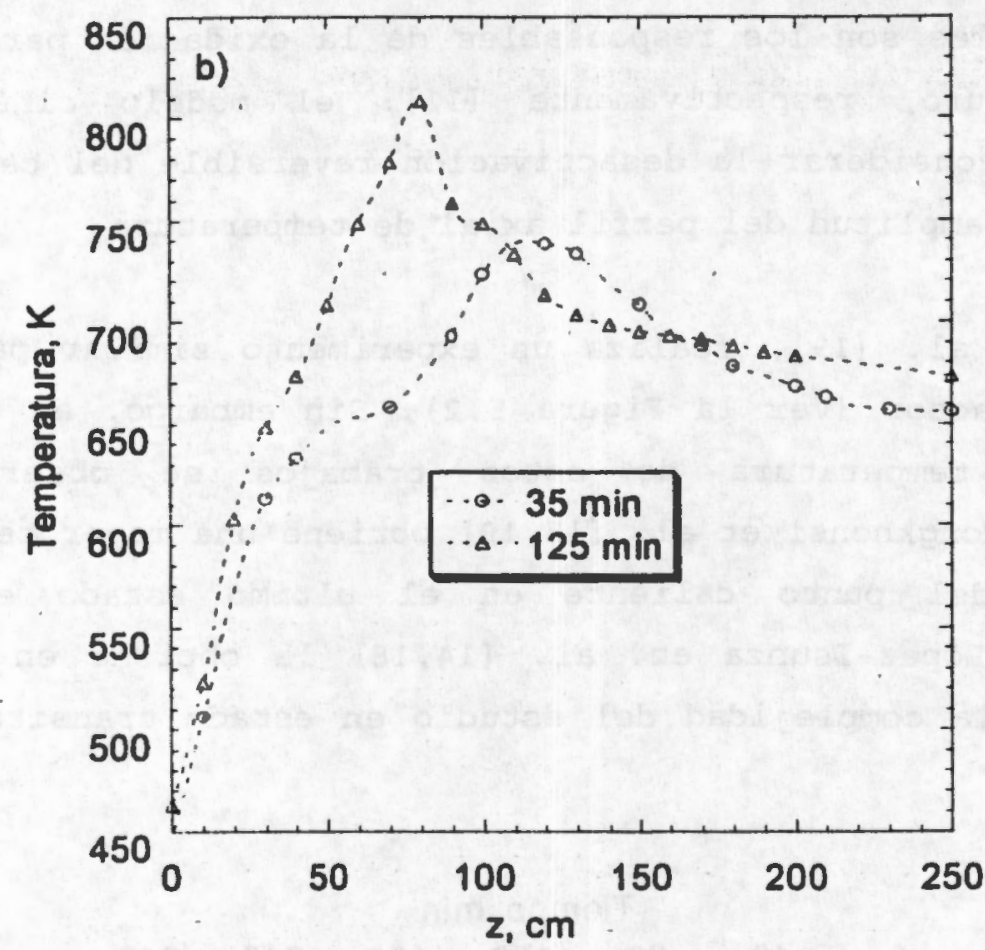

Figura 5.2. Continuación.

\subsection{Modelo cinético de la oxidación de OX a AF}

En el Apéndice I se propone un modelo cinético que podría predecir el comportamiento de este reactor tanto en estado estacionario como transitorio. Sin embargo, la evaluación de este modelo cinético necesitaría de un largo estudio teorico-experimental, y queda como trabajo a futuro. En este capitulo, se utiliza un modelo cinético que considera los mismos pasos de reacción empleados en el modelo de Calderbank et. al. [9] lo que permite emplear sus parámetros cinéticos. Asimismo, se considera de manera implicita la desactivación del catalizador en la dinámica oxido-reducción que sufre el catalizador.

El modelo cinético utiliza el esquema de reacción presentado en el Apëndice $I$. Este consideró dos tipos de sitios, los cuales se relacionan con el estado de oxidación del vanadio en la superficie: un estado de oxidación favorable a la oxidación parcial $\left(\mathrm{v}^{+5}\right)$ y otro a la 

de oxidarión parcial de oxileno a anhidrido frelico

oxidación total $\left(\mathrm{V}^{+3}\right)$ [11]. Así también, que la regeneración de los sitios reducidos depende de la temperatura y concentración que se tenga a lo largo del reactor. Es decir, una mayor concentración de ox indicaria una mayor cantidad de sitios reducidos, debido a la fuerte adsorción de hidrocarburos en la superficie del catalizador; mientras que, una mayor temperatura favorecería a la oxidación total de estos hidrocarburos adsorbidos, y por lo tanto una mayor cantidad de sitios oxidados. In este sentido si $\theta_{0}$ es el sitio responsable para llevar a cabo la oxidación parcial y $\theta_{\varepsilon}$ la oxidación total, se puede utilizar la el siguiente paso cinético para considerar tal fenómeno en la superficie del catalizador:

$\mathrm{O}_{2}+\mathrm{m} \theta_{\mathrm{r}} \underset{\mathrm{k}_{\mathrm{r}}}{\stackrel{\mathrm{k}_{0}}{\longrightarrow}} \mathrm{n} \theta_{0}$

Donde las velocidades de reacción se consideran de primer orden con respecto al hidrocarburo [8] .

$R_{j}=\theta_{0} k_{j} P_{n \in d}$ Para $j=1, \ldots, 6$

En donde:

$k_{j}=A_{j} \exp \left(-E a_{j} / R_{g} T\right)$ para $j=1, \ldots, 6$

El balance de sitios en la superficie del catalizador para considerar la desactivación reversible en la zona de reacción es:

$N_{=} \frac{d \theta_{0}}{d t}=\left[k_{0} \theta_{r}-\left\{k_{r}+\sum_{j=1}^{r} m_{j} k_{j} R_{g} T C_{n j}\right\} \theta_{0}\right]$

Esta Ecuación es muy sensible a variaciones en los valores de las constantes de re-oxidación, $k_{0}$ y reducción del catalizador, $k_{r}$. Variar la energía de re-oxidacion del catalizador esta relacionado directamente con el valor de estos parámetros. Si $\mathrm{E}_{\text {ao }}$ incrementa la 

de oxidación parcial de oxileno a anhidrido frilico

constante de re-oxidación disminuye $k_{0}=A_{0} e^{(- \text {Rao/RgT) }}$; por lo que, para tener sitios selectivos a la oxidación de hidrocarburos, la constante de re-oxidación, siempre debe ser mayor que la de reducción, por lo que $\mathrm{E}_{\mathrm{ar}}$ siempre es mayor que $\mathrm{E}_{\mathrm{ao}}$.

Se puede observar que si se considera el estado estacionario, se tiene nuevamente el modelo cinético de Calderbank et al. [8]:

$$
\theta_{3}=\frac{k_{0} P_{O_{2} s}}{k_{0} P_{O_{2,}}+\sum_{j=1}^{6} m_{j} k_{y} P_{n s y}}
$$

Para predecir la desactivación del catalizador a la entrada del lecho se utiliza la ecuación (4.28) y se consideran las ecuaciones (5.1-5.5) para predecir el comportamiento del catalizador en operaciones transitorias del reactor.

$$
a(z)=\frac{a_{0} \exp (p z)}{1-q a_{0}[1-\exp (p z)]}
$$

\subsubsection{Validación del modelo cinético en estudios en estado estacionario}

Para validar el modelo cinético se ajusta el comportamiento observado en el reactor en estado estacionario a las condiciones de operación del Exp-1 (ver Tabla 4.2; Capitulo 4); $Y$ se utilizan los parámetros geométricos $y$ de transporte mostrados en las Tablas 4.1 y 4.3 respectivamente (ver Capitulo 4). Para la simulación del reactor se considera un modelo pseudo-heterogéneo con dispersión axial en dos dimensiones espaciales $y$ se emplean los parámetros de transporte de calor $\left(k_{\text {er }} y h_{w}\right)$ estimados al considerar la aproximación de capa limite (HET-2D-PH) (ver el capítulo 4). Este modelo cinético se valido considerando dos casos: 

de oxidación parcial de oxileno a anhidrido frálico

1) Sim-1: Se utiliza la Ecuación (4.28) para considerar la desactivación reversible a la entrada del lecho, considerando que "q" es igual a uno.

$R_{j}=a(z) \theta_{0} k_{j} P_{n e j}$ para $j=1, \ldots, 6$

De los sitios que participan durante la reacción se conoce que:

Al $t=0: \theta_{0}=\theta_{\text {oss }}$

Y, durante la reacción:

$\theta_{0}+\theta_{x}=1$

2) Sim-2. Se manejan tres tipos de sitios durante la reaccion: los sitios que no participan durante ia reacción $\left(\theta_{d}\right)$, los sitios selectivos a la oxidación parcial $\left(\theta_{0}\right)$, y los sitios selectivos a la oxidación total $\left(\theta_{r}\right)$. De esta forma se tiene lo siguiente:

$R_{j}=\theta_{0} k_{j} P_{n=1}$

Al $t=0: \theta_{0}=\theta_{\text {ose }}$

Y durante la reacción:

$\theta_{0}+\theta_{r}+\theta_{d}=1$

Los sitios que no participan durante la reacción $\theta_{d}$, se consideran como $\theta_{d}=1-a(z)$ y $a(z)$ se obtiene con la Ecuación (4.28).

La Figura 5.3 muestra el ajuste del modelo para SIM-1. La Figura 5.3a muestra la comparación entre las predicciones de los perfiles de temperatura y concentración, y las observaciones del Exp-1. La Figura 

de oxidación parcial de oxileno a anhidrido ftallico

5.3b muestra el perfil de actividad que se tienen durante la operación del reactor: el perfil de sitios $\theta_{0}$, el perfil de $a(z), y$ para comparar el perfil de $\theta_{\mathrm{a}}$. Los resultados señalan lo siguiente:

Cuando no se predice la amplitud del perfil axial de temperatura, es decir al utilizar el modelo de Calderbank et al. sin considerar la desactivación en la superficie del catalizador, se obtiene el perfil $\theta_{3}$ (ver el capitulo 4), el cual difiriere del perfil de $\theta_{0}$ obtenido en el caso SIM-1. Esto permitio ajustar la magnitud $y$ posición del punto caliente, asi como la amplitud del perfil axial de temperatura observado en el reactor. El perfil de $\theta_{0}$ muestra que la mayor cantidad de sitios con un estado de oxidación menor $\theta_{r}$ se obtiene antes de la zona del punto caliente; lo cual se relaciona con las altas concentraciones de ox que se tienen en esta región. Esto valida teóricamente algunas de las ideas presentadas en la sección 4.6 para proponer el modelo cinético.

En la Figura 5.4 se muestra la comparación entre el perfil axial de temperatura ajustado al considerar el caso SIM-2 y las observaciones a las condiciones del Exp-1. La Figura 5.4b muestra el perfil de $\theta_{0}$ obtenidos durante la operación del reactor. Esta forma de considerar la desactivación reversible del catalizador ajusta adecuadamente la magnitud y posición del punto caliente, asi como la amplitud del perfil axial de temperatura.

Las dos formas (SIM-1 y SIM-2) de ajustar el comportamiento observado en el reactor son adecuadas. No obstante, SIM-1 proporciona más información sobre los perfiles de actividad en el lecho. En el Apéndice $K$ se presenta un estudio de la sensibilidad de los perfiles de temperatura, concentración y actividad del catalizador, al variar tanto la concentración de entrada de ox como la temperatura del baño de enfriamiento. 

de oxidación parcial de oxileno a anhidride fmlico
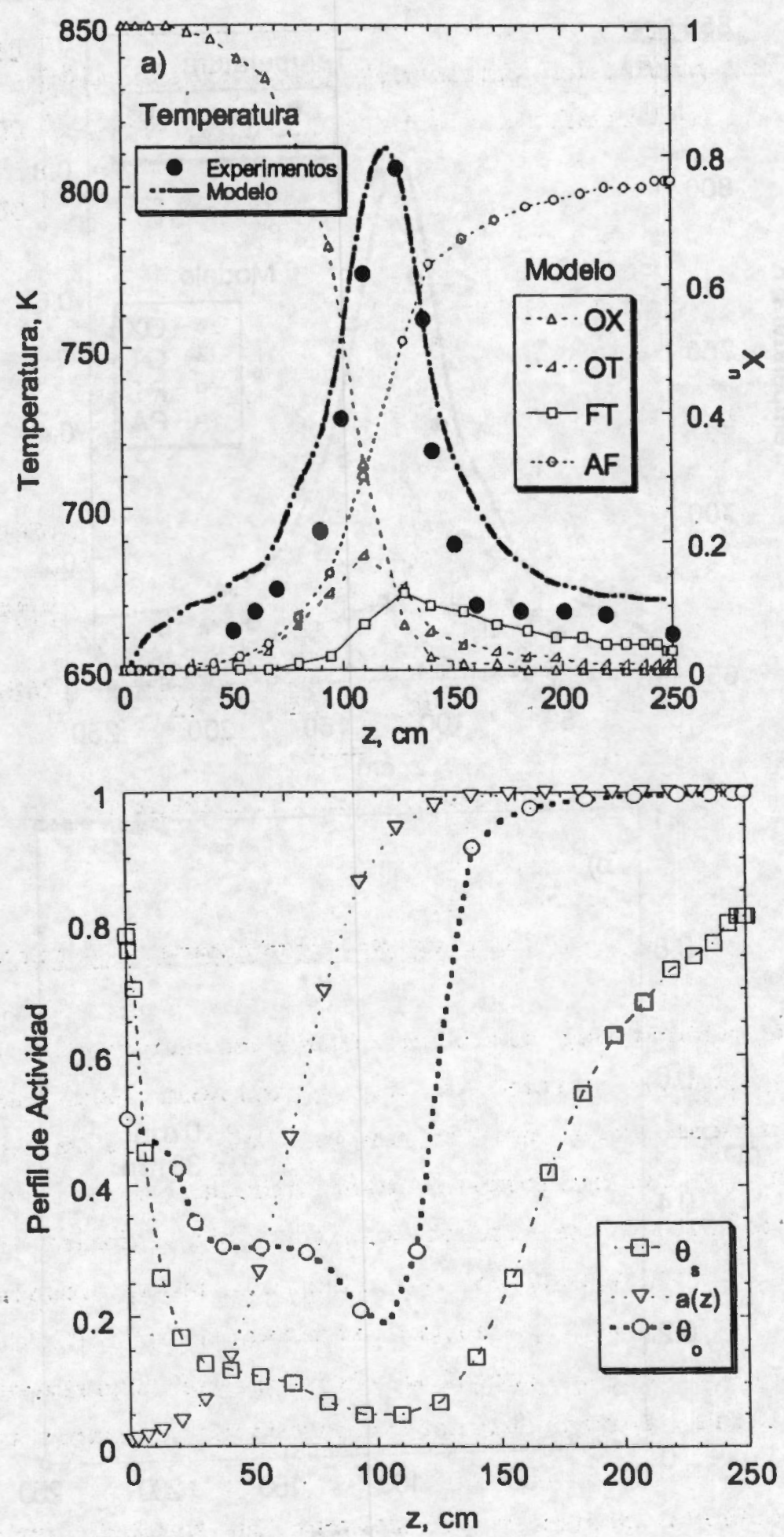

Figura 5.3 Ajuste de SIM-1 a las condiciones del Exp-1. a) Perfiles de temperatura y concentración y b) perfil de sitios presentes en la superficie del catalizador. 

de oxidación parcial de oxileno a anhidrido ftálico
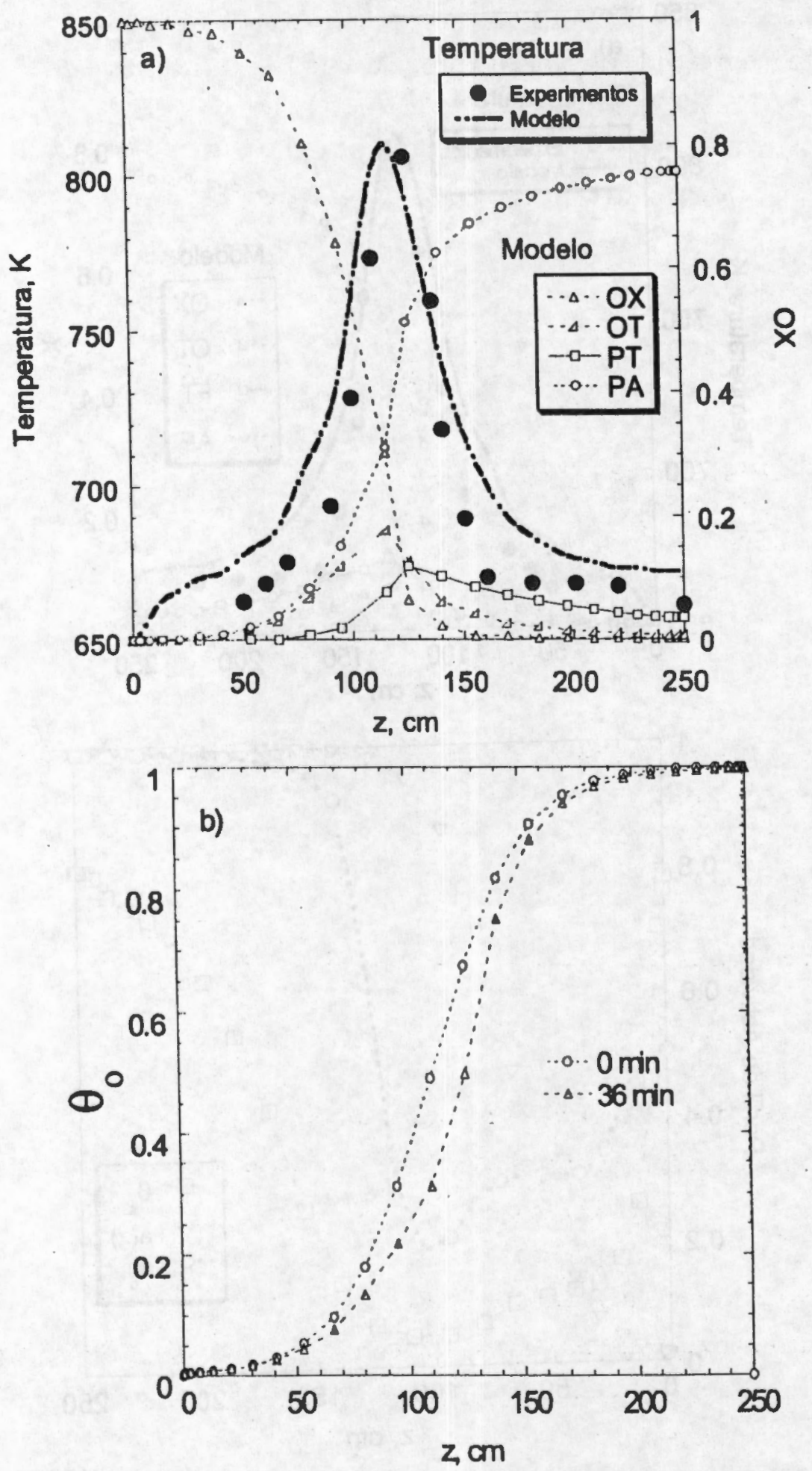

Figura 5.4 Ajuste de SIM-2 a las condiciones del Exp-1. a) Perfiles de temperatura y concentración y b) perfil de sitios presentes en la superficie del catalizador. 
5.3 Evaluación del modelo cinético en el estudio transitorio del reactor

En esta seccion se predice cualitativamente el comportamiento transitorio del reactor. Las condiciones de operación de arranque son: $T_{0}=T_{w}=660 \mathrm{~K}, Q=4 \mathrm{Nm} 3 / \mathrm{h}$ y $176 \mathrm{grox} / \mathrm{h}$. La temperatura del baño se incrementa en forma de rampa $10^{\circ}\left(1^{\circ} \times \mathrm{min}\right)$ despues de alcanzar el estado estacionario. Se utiliza el modelo HET-2D-PH para hacer la predicción del comportamiento transitorio.

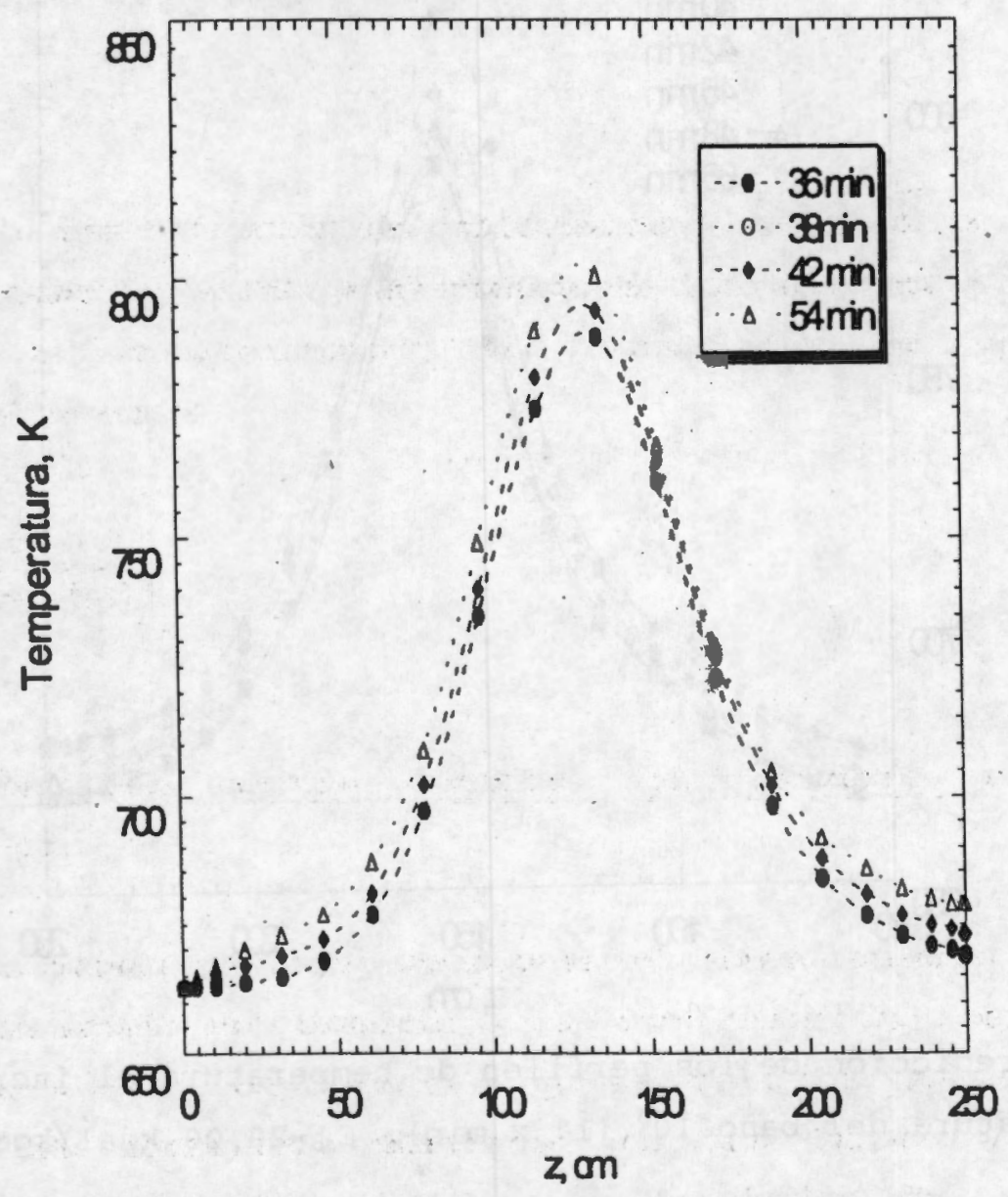

Figura 5.5. Predicción de los perfiles de temperatura al incrementar la temperatura del baño $10^{\circ}\left(1^{\circ} \mathrm{x} \mathrm{min}\right) ;$ y emplear el modelo cinético de Calderbank et al. [8]

La Figura 5.5 muestra el desplazamiento de los perfiles de temperatura al emplear el modelo cinético de Calderbank et al. [8]. En esta Figura, 

de oxidación parcial de oxileno a anhidrido frélico

el primer estado estacionario se tiene a los 36 minutos de reacción, mientras que el último se alcanza a los 18 minutos. Al comparar estos perfiles de temperatura con los observados en el reactor (ver la Figura 5.2b), se observa que es imposible predecir el desplazamiento del perfil de temperatura hacia la entrada del reactor.

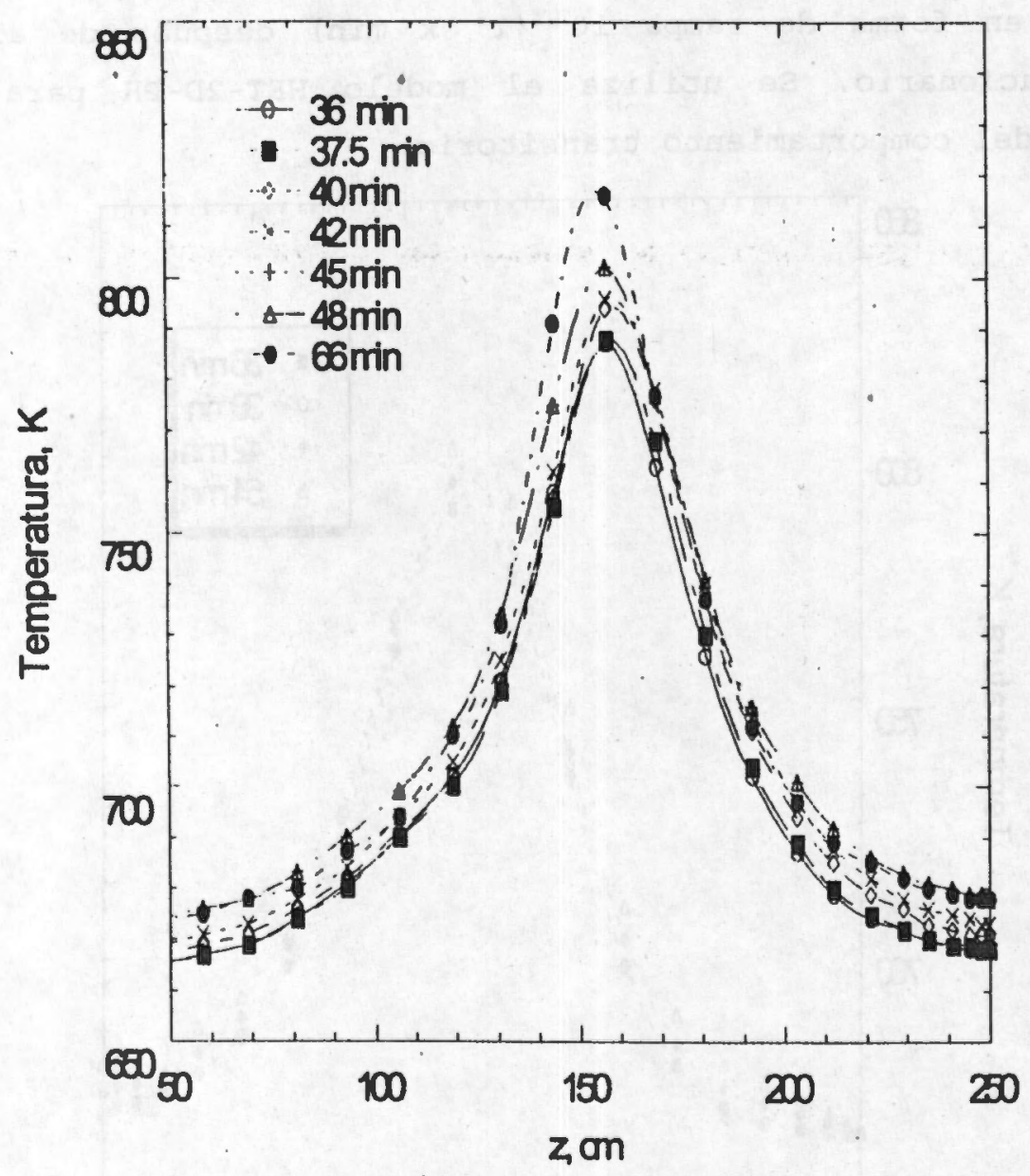

Figura 5.6. Predicción de los perfiles de temperatura al incrementar la temperatura del baño $10^{\circ}\left(1^{\circ} \times \mathrm{min}\right): \mathrm{E}_{\mathrm{ao}}=20,00 \mathrm{kcal} / \mathrm{kgmol}$.

La Figura 5.6 muestra la predicción del desplazamiento del perfil axial de temperatura al utilizar la propuesta SIM-1. Si se usa un valor de la $E_{\text {ar }}$ constante e igual a $45,000 \mathrm{Kcal} / \mathrm{Kgmol}$ y se utiliza un valor de la

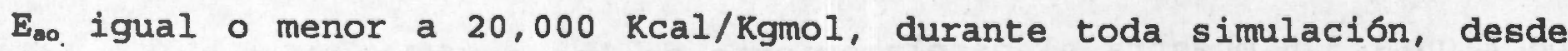
el arranque del reactor hasta el ultimo estado estacionario, no se 

de oxidación parcial de oxileno a anhidride fálice

puede predecir el desplazamiento del perfil de temperatura hacia la entrada del reactor, como se observo en la Figura 5.2b, y se habia sugerido en la literatura-[15] .

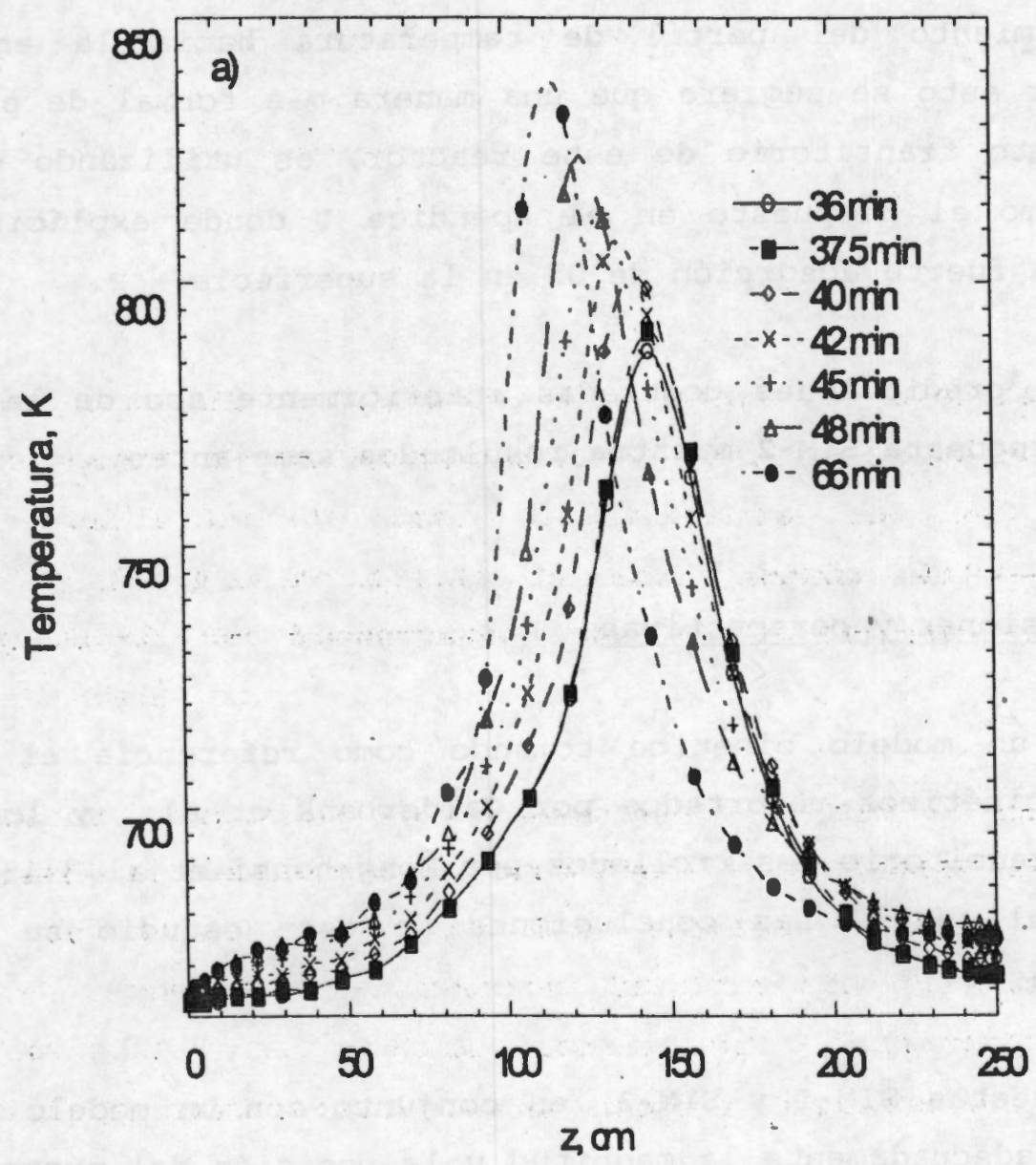

Figura 5.7. Predicción de los perfiles de temperatura al incrementar la temperatura del baño $10^{\circ}\left(1^{\circ} \times\right.$ min) y utilizar la propuesta SIM-1:

$$
E_{\Sigma_{0}}=15,00 \mathrm{kcal} / \mathrm{kgmol} \text {. }
$$

Para predecir el comportamiento transitorio observado en la Figura $5.2 b$, es necesario hacer lo siguiente: se utiliza desde el arranque del reactor hasta el primer estado estacionarlo (36 minutos), un valor de $k_{0}$ constante $\left(E_{a 0}=20,000 \mathrm{Kcal} / \mathrm{Kgmol}\right)$; al incrementar la temperatura del baño, $k_{0}$ mayor debe ser mayor, esto se logra cuando la $E_{a \circ}$ es menor a $20,000 \mathrm{Kcal} / \mathrm{Kgmol}$. La Figura 5.7 muestra el comportamiento transitorio del perfil axial de temperatura al mover $\mathbf{E}_{\mathbf{a}}$ para incrementar el valor 

de oxidación parcial de oxileno a anhidrido falico

$k_{0}$. Esta metodología es una manera informal e implicita de considerar la adsorción de ox en la superficie del catalizador y su oxidación total. Además que, el uso de una expresión como la (4.28), que considera la desactivación reversible a la entrada del rector, limita el desplazamiento del perfil de temperatura hacia la entrada del reactor. Por esto se sugiere que una manera más formal de predecir el comportamiento transitorio de este reactor, es utilizando un esquema cinética como el propuesto en el Apéndice I donde explícitamente se considera la fuerte adsorción de ox en la superficie.

Si bien las predicciones mostradas anteriormente son de la propuesta SIM-1, la propuesta SIM-2 muestra resultados semejantes.

\subsection{Conclusiones $Y$ perspectivas}

Se utilizo un modelo cinético tomando como referencia el esquema y parámetros cinéticos reportados por Calderbank et al., y los estudios en estado transitorio desarrollados por Mongkhonsi et al. [15] y LopezIsunza et al. [18]. Las conclusiones de este estudio se resumen a continuacion:

a) Las propuestas SIM-1 Y SIM-2, en conjunto con un modelo HET-2D-PH, predicen adecuadamente la magnitud y la posición del punto caliente, asi como la amplitud del perfil axial de temperatura. Esto se logro toda vez que el modelo cinético considera los fénómenos de desactivación reversibles en términos de considerar la dinámica oxido-reducción en el catalizador. Asimismo se observo que la amplitud del perfil axial de temperatura se relaciona directamente con el perfil de actividad que se establece a lo largo del lecho.

b) El considerar la dinamica oxido-reducción en el catalizador es una manera informal y cualitativa de considerar la desactivación reversible en el mismo. En donde los parámetros $k_{0}$ y $k_{r}$ juegan un 

de oxidación parcial de oxileno a anhidrido fálice

papel importante para predecir el comportamiento transitorio del perfil axial de temperatura.

c) El considerar una expresión empirica que considere la desactivación a la entrada del lecho, $a(z)$, limita el desplazamiento de los perfiles de temperatura hacta la entrada del lecho $y$ nuevas expresiones deben ser consideradas.

Finalmente, se sugiere utilizar un modelo cinetico que considere un esquema de reacción como el que se discute en el Apéndice $I, y$ con éste intentar simular el comportamiento transitorio del reactor. 
Capitulo 6.

Las conclusiones generales

Capítulo 6.

Conclusiones 


\section{Conclusiones}

En este trabajo se estudio el comportamiento, en estado estacionario y transitorio, de un reactor a escala industrial, de lecho fijo, en donde tiene lugar una reacción de oxidación de o-xileno a anhídrido ftálico. Los principales problemas en la simulación de este reactor se relacionaron con la no adecuada descripción de los fenómenos de transporte de calor en el reactor, y de desactivación en la superficie del catalizador. Para tener una adecuada predicción de los perfiles de temperatura y concentración observados en este reactor se realizaron estudios independientes sobre: la hidrodinámica; el transporte de calor en ausencia de reacción; $y$ de la reacción en estado estacionario $y$ transitorio. Cada uno de estos estudios dio lugar a diferentes conclusiones, comentadas al final de cada capitulo. Aquí únicamente remencionan las principales aportaciones que se tuvieron en esta tesis doctoral:

Se hace un estudio de diferentes modelos hidrodinámicos, derivados de uno general, que han sido utilizados con gran incertidumbre en la literatura. Con esto se sugiere un modelo hidrodinámico que podría predecir los campos de velocidad en un lecho empacado con una relación de $d_{c} / d_{p}<5$. Se encuentra que la estimación de los fenómenos inerciales $y$ viscosos es fundamental para predecir.los campos de velocidad en nuestro sistema. Estos fenbmenos se pueden estimar a partir de datos de caida de presión o de velocidad obtenidos en el mismo sistema experimental. Esto permitio omitir el empleo de la viscosidad efectiva del modelo hidrodinámico.

El empleo de un lecho a escala de laboratorio, para estimar los parámetros de transporte de calor, predijo adecuadamente los perfiles de temperatura en un reactor a escala industrial. La consideración de isotropía o no isotropía, en la estimación de $k_{a x} y h_{w}$, predice los perfiles de temperatura tanto en el lecho de escala de laboratorio como industrial. La consideración de isotropia permitio eliminar algunos problemas estadisticos que se tenian al estimar ambos parámetros de 
transporte de calor utilizando un mismo conjunto de experimentos. Asimismo, la predicción de los perfiles de temperatura empleando la aproximación clásica es tan buena como los modelos que incorporan los perfiles de velocidad. Los valores estimados de $k_{a x} y h_{w}$, bajo las consideraciones de los tres casos (A y B), se encuentran dentro de los limites de confiabilidad, sin embargo, éstos presentan importantes diferencias ente un caso $y$ otro, lo que afecta sensiblemente la predicción de los perfiles de temperatura en la simulación del reactor catalitico. Se sugiere, que si no se cuenta con medidas de temperatura de la pared interna del lecho, se puede emplear la aproximación de capa límite para estimar $k_{\text {er }}$ y $h_{w}$, la cual da adecuadas predicciones de los procesos de transporte de calor puros y con reacción.

El comportamiento observado en el reactor fue predicho toda vez que $k_{e x}, k_{o r} y h_{w}$ se estimaron al considerar la hidrodinámica, e isotropia $\sigma$ no-isotropia en el estudio de transporte de calor puro. Sin embargo, el considerar el modelo hidrodinámico en el modelamiento del reactor no predice correctamente los perfiles de temperatura y concentración observados en el reactor. Esto sugiere que los parámetros de transporte de calor contienen implicitamente el efecto de la hidrodinámica. Por otro lado, el considerar la desactivación del catalizador es necesario en la predicción de experimentos en estado estacionario. Todos los modelos del reactor predicen el comportamiento experimental del sistema de reacción; sin embargo, el perfil de actividad es diferente en cada caso. Esta diferencia permitio establecer un criterio para discriminar entre los modelos utilizados; el modelo pseudo-heterogéneo en dos dimensiones espaciales es el más adecuado para simular el reactor estudiado. Finalmente, al utilizar los resultados que se tienen en el estudio de la superficie del catalizador $y$ experimentos en un reactor industrial, se planteo un esquema de reacción que podría describir el comportamiento del catalizador.

Se planteo un modelo cinético basado en el esquema y parámetros cinéticos propuestos por Calderbank et. al. Este considera los fenómenos de desactivación reversibles en la superficie del 
Capitulo 6.

Las conclusiones generales

catalizador, con lo que se logra predecir los resultados observados en el reactor, en el estado estacionario, y cualitativamente en el transitorio; lo cual no sucedía cuando se empleaba únicamente el modelo cinético de Calderbank et al. 


\section{Referencias bibliográficas}

1. Taylor G., 1919. Heat of reaction of ammonia oxidation. The Journal of Industrial and Engineering Chemistry 11, 1121.

2. Wilson K. B., 1946. Calculation and analysis of longitudinal temperature gradients in tubular reactors. Trans. Inst. Chem. Engrs. (London) 24, 77.

3. Tasker G. J. H., 1946. The calculation of heat transfer and reaction rate in catalyst beds. Application of theory to phthalic anhydride pilot plant data. Trans. Inst. Chem. Engrs. (London) $24,84$.

4. Loftus J. And Satterfield C., 1965. Kinetics of the homogeneous partial oxidation of o-xylene vapor by air. I\&EC Process Design and Development 4, 102.

5. Loftus J. And Satterfield C., 1965. Mechanism of homogeneous gas-phase partial. oxidation of o-xylene. I\&EC Process Design and Development 60(3), 1966 .

6. Mars P. and van Krevelen D.W., 1954. Oxidation carried out by means of vanadium oxide catalysts. Special supplement to Chemical Engineering Science 3, 41.

7. Simard G.L., steger J.F., Arnott R.J., and Siegel L.A., 1955. Vanadium oxides as oxidation catalysts. Industrial and Engineering Chemistry $47(7), 1424$.

8. Calderbank P.H., Chandrasekharan K. and Fumagalli C., 1977. The prediction of the performance of packed-bed catalytic reactors in the air-oxidation of o-xylene. Chemical Engineering Science $32(12), 1435-1443$.

9. Vanhove D. and Blanchard M., 1976. Catalytic oxidation of oxylene. Journal of Catalysis 36,6 .

10. Elnashaie S.; Fouad M.; Elshishini S., 1990. The use of mathematical modeling to investigate the effect of chemisorption on the dynamic behavior of catalytic reactors. Partial oxidation of o-xylene in fluidized beds. Mathematical and Computer Modeling 13(5), 11. 
11. Bond G.C., 1997. What limits the selectivity attainable in the catalysed oxidation of o-xylene to pthalic anhydride. J. Chem Tech. Biotechnol 68,6 .

12. Anastasov A. 2002. A study of the influence of the operating parameters on the temperature of the hot spot in a fixed bed reactor. Chemical Engineering Journal, 86(3), 287.

13. Papageorgiou J.N. and Froment G.F., 1996. Pthalic anhydride synthesis. Reactor optimization aspects. Chemical Engineering Science $51(10), 2091$.

14. Lopez-Isunza F. 1983. Steady State and Dynamic Behavior, of an Industrial Fixed Bed Catalytic Reactor. Ph.D. Thesis, Imperial College, University of London.

15. Mongkhonsi T., 1994. Ph.D. thesis, Imperial College University of London.

16. Abi C.F., Ph.D. 1986. Ph.D. thesis, Imperial College; University of Iondon.

17. Skrzypek J.; Grzesik M.; Galantowicz M.; Solikski J., 1985. Kinetics of the catalytic air oxidation of o-xylene over a commercial $\mathrm{V}_{2} \mathrm{O}_{5}-\mathrm{TiO}_{2}$ catalyst. Chemical Engineering Science $40(4), 611$.

18. Lopez-Isunza F. and Kershenbaum L.S, 1992. The role of reversible changes in catalyst activity in the observed multiple steady stated during partial oxidation dymamics. Chemical Engineering Science $47(9-11), 2817$.

19. Mongkhonsi T.; Kershenbaum L., 1998. The effect of deactivation of a $\mathrm{V}_{2} \mathrm{O}_{5} / \mathrm{TiO}_{2}$ (anatase) industrial catalyst on reactor behavior during the partial oxidation of o-xylene to phthalic anhydride. Applied Catalysis A General, 170(1), 33.

20. www.belembassy.org/poland/pol/economics/lakokraskapl.pdf

21. Ying-Sheng C., Lopez-Isunza F., Mongkhons1 T. and Kershenbaum I., 1993. Estimation of catalyst activity profiles in fixed-bed reactors with decaying catalysts. Applied Catalysis A: General $106(2), 193-199$. 
22. Chandrasekharan K.; Calderbank P.H., 1980. Kinetics of the catalytic air-oxidation of o-xylene measured in a tube-wallcatalytic reactor. Chemical Engineering science $35(1-2), 341$.

23. Anastasov A.I.. 2003. An investigation of the kinetic parameters of the o-xylene oxidation process carried out in a fixed bed of high-productive vanadia-titania catalyst. Chemical Engineering Science 58(1), 89-98.

24. Dias C.R., Portela M.F., Bond G.C., 1996. Oxidation of O-xylene to phthalic anhydride over $\mathrm{V}_{2} \mathrm{O}_{5} / \mathrm{TiO}_{2}$ catalysts. Mathematical modelling study and analysis of the reaction network. Journal of Catalysis 164 (2) . 276-287.

25. Wainwright M.S. and Foster N.R., 1979. Catalysts, kinetics, and reactor design in phthalic anhydride synthesis. Catalysis Review: Science and Engineering.

26. Mongkhonsi T.; Lopez-Isunza H.F.; Kershenbaum L.S., 1992. The distortion of measured temperature profiles in fixed-bed reactors. Trans I ChemE 70 (A), 255.

27. Castillo-Araiza, Jiménez-Islas H. and López-Isunza F., 2007. Heat transfer studies in packed bed catalytic reactors of low tube/particle diameter ratio. Industrial and Engineering Chemistry Research 46,7426-7435.

28. Elnashaie S.S.E.H and Elshishini S.S., 1993. Modelling simulation and optimization of industrial fixed bed catalytic reactors, Amsterdam.

29. Nikolov V. A.: Anastasov A. I., 1992. Influence of the inlet temperature on the performance of a fixed-bed reactor for oxidation of o-xylene into phthalic anhydride. Chemical Engineering Science 47 (5), 1291.

30. Papageorgiou J.N.; Froment G.F.,. 1995. Simulation models accounting for radial voidage profiles in fixed-bed reactors. Chemical Engineering Science 50 (19), 3043.

31. Cheng Y.S., Lopez-Isunza F., Mongkhonsi T. and Kershenbaum L., 1993. Estimation of catalyst activity profiles in fixed-bed reactors with decaying catalysts. Applied Catalysis A: General 
Referencias bibliograficas

$106(2), 193-199$.

32. Cheng Y. S; Abi C. F.; Kershenbaum L. S., 1996. On-line estimation for a fixed-bed reactor with catalyst deactivation using nonlinear programming techniques. Computers chem. Engng. $20,793$.

33. Cheng Y.S.; Mongkhonsi T.; Kershenbaum L. S., 1996. Nonlinear dynamic estimation for a fixed-bed reactor with decaying catalysts. Chemical Engineering Science 51(10), 1909.

34. Anastasov A., 2005. The behaviour of a low-productive nonpretreated $\mathrm{V}_{2} \mathrm{O}_{5}-\mathrm{TiO}_{2}$ catalyst for oxidation of o-xylene to phthalic anhydride. Chemical Engineering Journal 109, 57.

35. Nikolov V., klissurski D. and Anastasov A., 1991. Phthalic anhydride from o-xylene catalysis: Science and Engineering. Catalysis Review- science and Engineering $33(3,4), 319$.

36. Elshishini S.S., Elnashaie S.S. and El-Rifaie M., 1987. Multiplicity of the steady state in fluidized bed reactorspartial oxidation of o-xylene. Computer in Chemical Engineering II (2), 95 .

37. Tsotsas E. and Schlunder E., 1990. Heat transfer in packed beds with fluid flow: remarks on the meaning and the calculation of a heat transfer coefficient at the wall. Chemical Engineering Science $45(4), 819-837$.

38. Castillo A. C. O., 2004. Estudios de Transferencia de Calor en un Lecho Empacado. Tesis de Maestría, Universidad Autónoma Metropolitana - Iztapalapa, México D.F.

39. Nijemeisland, M., Dixon, A.G., stitt, E.H., 2004. Catalyst design by CFD for heat transfer and reaction in steam reforming. Chemical Engineering Science 59, 5185-5191.

40. Nijemeisland, M., Dixon, A.G., 2001. Comparison of CFD simulations to experiment for convective heat transfer in a gas-solid fixed bed. Chemical Engineering Journal 82, 231-246.

41. Lerou, J.J., Froment, G.F., 1977. Velocity, temperature and conversion profiles in fixed bed catalytic reactors. Chemical Engineering Science 32, 853-861. 
Referencias bibliográficas

42. Lerou J.J.; Froment G.F., 1978. Estimation of heat transfer parameters in packed beds from radial temperature profiles. The Chemical Engineering Journal 15(3), 233.

43. MCGreavy C., FoumenY E.A., and Javed K.H., 1986. Characterization of transport properties for fixed bed in terms of local bed structure and flow distribution. Chemical Engineering Science 41(4), 787-797.

44. Kufner ' $R$. and Hofmann H., 1990. Implementation of radial porosity and velocity distribution in a reactor model for heterogeneous catalytic gas phase reactions. Chemical Ẹngineering Science 8, 2141-2146.

45. Morales M. Spinn C. W., and Smith J. M., 1951. Velocities and effective thermal conductivities in packed beds. Engineering and Process Development 43 (1), 225-232.

46. Benenati R.F. and Brosilow C.B., 1962. Void fraction distribution in beds of spheres. Journal of American Institute of Chemical Engineers 8(3), 359-.361.

47. Govindarao V.M. and Froment G.F., 1986. Voidage profiles in packed bed of spheres. Chemical Engineering Science 41, 533539.

48. Govindarao V.M. and Ramrao K.V., 1988. Prediction of location of particles in the wall region of a randomly packed bed of spheres. Chemical Engineering Science 43, 2544-4545.

49. Bear J., 1988. Dynamics of fluids in porous media, Dover Publications Inc., N.Y.

50. Govindarao V.M., Ramrao K.V. and Rao A.V., 1992. Structural characteristics of packed bed of low aspect ratio. Chemical Engineering Science 47, 2105-2109.

51. Stanek V, 1994. Fixed Bed Operations. Ellis Horwood, N. Y.

52. Kaviany M. , 1995. Principles of heat transfer in porous media. Springer-Verlag, N. Y.

53. Wang X., Thauvin F., Mohanty K.K., 1999. Non-Darcy flow through anisotropic porous media. Chemical Engineering science 54, 1859-1869. 
54. Winterberg. M., Tsotsas, 2000. Impact of tube-to-particle diameter ratio on pressure drop in packed beds. Journal of American Institute of Chemical Engineers $46(5), 1084-1088$.

55. Denys K. F., 2003. Flow of polymer solutions through porous media. Technische Universitelt Delft, P.h. Thesis, Holanda.

56. Giese M., Rottshafer K. and Vortmeyer D., 1998. Measured and modeled superficial flow profiles in packed beds with liguid flow. AIChe Journal 44, 484-490.

57. Magnico P., 2003. Hydrodynamic and transport properties of packed beds in small tube-to-particle diameter ratio: pore scale simulation using an Eulerian and Lagrangian approach. Chemical Engineering Science 58, 5005-5024.

58. Freund H., Zeiser T., Huber F., Klemm E., Brenner G., Durst F. and Emig G., 2003. Numerical simulations of single phase reacting flows in randomly packed fixed-bed reactors and experimental validation. Chemical Engineering Science 58, 903910.

59. De Klerk A., 2003. Voidage variation in packed beds at small column to particle diameter ratios. Journal of American Institute of Chemical Engineers 49, 2022-2029.

60. Eisfeld B., Schnitzlein K., 2005. A new pseudo-continuous model for the fluid flow in packed beds. Chemical Engineering science 60, 4105-4117.

61. Daszkowski T., Eigenberger G., 1992. A re-evaluation of fluid flow, heat transfer and chemical reaction in catalyst filled tubes. Chemical Engineering Science 47, 2245-2250.

62. Foumeny E.A. \& Ma J., 1994. Non-Darcian non-isothermal compressible flow and heat transfer in cylindrical packed beds. Chemical Engineering Technology 17, 50-60.

63. Bey O.: Eigenberger G.; 1997. Fluid flow and through catalyst filled tubes. Chemical Engineering Science 52, 1365-1376.

64. Logtenberg S.A. and Dixon A. G., 1998. Computational fluid dynamics studies of the effects of temperature-dependent physical properties on fixed-bed heat transfer. Industrial and 
Referencias bibliográficas

Engineering Chemistry Research 37(3), 739-747.

65. Logtenberg S.A., Nijemeisland M. and Dixon A. G., 1999. Computational fluid dynamics simulations of fluid flow and heat transfer at the wall-particle contact points in a fixed-bed reactor. Chemical Engineering Science 54 (13-14), 2433-2439.

66. Winterberg M., Tsotsas E., Krischke A., Vortmeyer D., 2000. A simple and coherent set of coefficients for modeling of heat and mass transport with and without chemical reaction in tubes filled with spheres. Chemical Engineering Science 55, 967-979.

67. Nassehi V., 1997. Modeling of combined Navier-Stokes and Darcy flows in crossflow. Chemical Engineering Science 536, P.p. 12531265 .

68. Nijemeisland M., 2000. Verification studies of Computational fluid Dynamics in fixed bed heat transfer. Faculty of the Worcester Polytechnic Institute, Master Thesis.

69. Darcy H., 1856. Les Fontaines Publiques de la Ville de Dijon, Dalmon, Paris.

70. Brinkman H. C., 1947. A calculation of the viscous force exerted by a flowing fluid on a dense swarm of particles. Applied Scientific Research (A1), 27-34.

71. Jiménez-Islas H., López-Isunza F. and Ochoa-Tapia J. A., 1999. Natural convection in a cylindrical porous cavity with internal heat source: a numerical study with Brinkman-extended Darcy model. International Journal of Heat and Mass Transfer $42(22)$, 4185-4195.

72. Nield \& Bejan, 1992. Convection in porous media. SpringerVerlag, N.Y.

73. Ergun S., 1952. Fluid flow through packed columns. Chemical Engineering Progress 48, 89-94.

74. Forchheimer, 1935. Tratado de hidrálica. Ed. Labor: Barcelona Spain. .

75. Jiménez-Islas H., 1998. Modelamiento matemático de los procesos de transferencia de momento, calor y masa en medios porosos. UAM-Iztapalapa, Tesis Doctoral, México. 
76. Castillo-Araiza C. and López-Isunza F., 2007. Hydrodynamic models for packed beds with low tube-to-particle diameter ratio. International Journal of Chemical Reactor Engineering. To be published.

77. Stewart W.E., M. Caracotsios M., 1995. Sorensen J.P, Parameter Estimation for Multiresponse Data. GREGPAK.

78. Finlayson B.A., 1980. Nonlinear analysis in Chemical Engineering. McGraw-Hill: New York.

79. Lapidus L., Seinfeld J.H., 1971. Numerical solution of ordinary differential equations. Academic Press, : New York.

80. Eisfeld B., Schnitzlein K., 2001. The influence of confining walls on the pressure drop in packed beds. Chemical Engineering Science 56, P.p. 4321-4329.

81. Niven R.K., 2002. Physical insight into Ergun and Wen \&Yu equation for fluid flow in packed bed and fluidized beds. Chemical Engineering Science 57, P.p. 527-534.

82. Macdonald I.F.. El-Sayed M.S, , Mow K., and Dullien F.A.L., 1979. Flow through porous media- The Ergun equation revisited. Industrial Engineering Chemical Fundaments 18(3), p.p. 199-208.

83. Felice R. D., Gibilaro L.G., 2004. Wall effects for the pressure drop in fixed beds. Chemical Engineering Science 59, P.P. $3037-3040$

84. Castillo-Araiza C.0., 2006. Estudio hidrodinámico de lechos empacados. XXVII Encuentro Nacional AMIDIQ, México.

85. Delmas H. and Froment G.F., 1988. A simulation model accounting for structural radial no uniformities in fixed bed reactors. Chemical Engineering Science 43, 2281-2287

86. Hlavacek V., 1970. Aspects in design of packed bed reactors. Industrial and Engineering Chemistry 62 (7), 8-26.

87. Froment G. F., 1967. Fixed bed catalytic reactor. Industrial Engineering Chemical 59, 18.

88. Smith J.M., 1973. Heat transfer in fixed-bed reactors. The Chemical Engineering Journal 5(2), 109.

89. Derk O. and Anthony G.D., 1997. Effect of the wall Nusselt 
Referencias bibliogrạficas

number on the simulation of catalytic fixed bed reactors. Catalysis Today 35, 435.

90. Colburn A.P., 1931. Transfer and pressure drop in empty baffled and packed tubes. Industrial and engineering chemistry $23(8)$, 910.

91. Bunnell D.G.; Irvin H.B.; Olson R.W.; Smith J.M., 1949. Effective thermal conductivities in gas-solid system. Industrial and Engineering Chemistry 41(9), 1977.

92. Kunii D.; Smith J.M., 1961. Heat transfer characteristics of porous rocks II, AIChe Journal, 29.

93. De Wasch A.P.; Froment G.F., 1972. Heat transfer in packed beds. Chemical Engineering Science 27, 567.

94. Tsang, T.H.; Edgar, T.F.; Hougen, J.O., 1976. Estimation of heat transfer parameters in a packed bed. The Chemical Engineering Journal 11, 57.

95. Li C.H.; Finlayson B.A., 1977. Heat transfer in packed beds-a reevaluation. Chemical Engineering Science 32, 1055.

96. Schlunder E.U., 1978. Transport phenomena in packed bed reactors, 1878. Chemical Reactor Engineering- Houston. ACS Symposium Series 65, 110.

97. Dixon A.G., Paterson W. R. and Cresswell D. L., 1978. Heat transfer in packed beds of low tube/particle diameter ratio. Chemical Reactor Engineering- Houston. ACS Symposium Series 65, 238-253.

98. Dixon A.G; Cresswell D.L., 1979. Theoretical prediction of effective heat transfer parameter in packed beds. Journal of American Institute of Chemical Engineers $25(4), 663$.

99. Michelsen M., 1979. Estimation of heat transfer parameters in packed beds from radial temperature measurements. The Chemical Engineering Journal 18, 67.

100. Lerou, J.J.; Froment, G.F., 1979. Reply to comments by A. G. Dixon and $D$. L. Cresswell on "Estimation of heat transfer parameters in packed beds from radial temperature profiles". The Chemical Engineering Journal 17(3), 248. 
101. Froment and Bischoff. Chemical Reactor Analysis and Design. J. Wiley: New York 1979.

102. Kulkami B.D.; Doraiswamy I.K., 1980. Estimation of effective transport properties in packed bed reactors. Catalysis Review: Science and Engineering $22(3), 431$.

103. Cresswell D.L.; Dixon A.G., 1982. Reply to comments by Vortmeyer and Berninger on the paper "Theoretical prediction of effective heat transfer parameter in packed beds (AIChe J., 25, 663,1979 )". AIChe Journal 28 (3), 51.

104. Vortmeyer D.; Berninger R., 1982. Comments on the paper, theoretical prediction of effective heat transfer parameter in packed beds by Cresswell D. L., Dixon A. G. (AIChe J., 25,663, 1979)". AIChe Journal, 28 (3), 508.

105. Wakao N.; Kaguei $S$. Heat and mass transfer in packed beds. Science Publishers Ltd; New York 1983.

106. Dixon A.G., 1985. The Length Effect on Packed Bed Effective Heat Transfer Parameters. The. Chemical Engineering Journal 31, $163-173$.

107. Dixon A. G., Cresswell. D. L., 1986. Effective heat transfer parameters for transient packed-bed models. Journal of American Institute of Chemical Engineers 32 (5), 809.

108. Borman P.C.; Borkink J.G.H.; Westerterp K.R., 1992. Heat transport in a wall heated tubular packed bed reactor. at elevated pressures and temperatures. Chem. Eng. Comm. 114, 17.

109. Tordanidis A.A., Mathematical Modeling of Catalytic Fixed Bed Reactors. P.h. Thesis, University of Twente 2002

110. Dixon A.G., van Dongeren J., 1998. The influence of the tube and particle diameters at constant ratio heat transfer in packed beds. Chemical Engineering and Processing 37, 23-32.

111. Cheng $z$. and Yuan W., 1997. Estimating radial velocity of fixed beds with low tube-to-particle diameter ratios. AIChE Journal $43(5), 1319-1324$. 
Referencias bibliográficas

112. Winterberg M., Tsotsas E., 2000. Modelling of heat transport in beds packed with spherical particles for various bed geometries and/or thermal boundary conditions. Int. J. Therm. Sci. 39, 556-570

113. Wilhelmus G. Heat and mass transport in tubular packed bed reactors. Ph. D. Thesis, University of Twente 2002 .

114. Mei H., Li C., Liu H., 2005. Simulation of heat transfer and hydrodynamics for metal structured packed bed. Catalysis Today 105. 689-696.

115. Wen D.; Ding Y., 2006. Heat transfer of gas flow through a packed bed. Chernical Engineering Science, 61, 3532.

116. Yagi S.; Kunii D. and Wakao N., 1960. Studies on axial effective thermal conductivities in packed beds. Alche Journal, 543 .

117. Vortuba J.; Hlavacek V.; Marek M., 1972. Packed bed axial. thermal conductivity. Chemical Engineering Science 27, 18451851 .

118. Vortmeyer D.; Adam W., 1984. Steady-state measurements and analytical correlations of axial effective thermal conductivities in packed beds at low gas flow rates. International Journal Heat Mass Transfer 27 (9), 1465-1472.

119. Hans T.A., 1999. The effective thermal conductivity of saturated porous media. University of Minnesota. Master Thesis, US.

120. Dixon A.G and Cresswell D.L., 1979, Comments on "Estimation of heat transfer parameters in packed beds from radial temperature profiles" by J. J. Lerou and G. F. Froment. The Chemical Engineering Journal 17(3), 247-249.

121. Legawlec b. and Ziolkowski D., 1995. Mathematical simulation of heat transfer within tubular flow apparatus with packed bed by a model considering system inhomogeneity. Chemical Engineering Science $50(4), 673-683$. 
Referencias bibliográficas

122. Tsotsas E.; Schlunder E. U., 1994. Comment on studies on axial dispersion in fixed beds (D. J. Gunn, Trans. Inst. Chem. Eng., 47 (1969) T351; D. J. Gunn, Chem. Eng. Process., 32 (1993) 333). Chemical Engineering and Processing, 33, 107.

123. Beek J., and Singer E., 1951. Chem. Engng Prog. Symp. Ser. 47, 534.

124. Patrick E., Rinaldo S. and Harriot P., 1988. Heat transfer in packed tube reactors. Industrial Engineering Chemical and Research (27), 226.

125. Finlayson B., 1971. Packed bed reactor analysis by orthogonal collocation. Chemical Engineering Science 26, 1081-1091.

126. Levenspiel O., 1999. Chemical reactor engineering. Ind. Eng. Chem. Res. 38, 4140 .

127. Karim A., Bravo J., Datye A., 2005. Nonisothermality in packed bed reactors for steam reforming of methanol. Applied Catalysis A: General 282, 101.

128. Ramirez J.F. and Calderbank P.H., 1977. The oxidation of benzene in packed catalyst beds. The Chemical Engineering Journal, 14, 49.

129. Maestri M., Beretta A., Groppi G. Tronconi E. and Forzatti P., 2005. Comparison among structured and packed-bed reactors for the catalytic partial oxidation of $\mathrm{CH}_{4}$ at short contact times. Catalysis Today 105, 709.

130. Hlavacek V. and Hofmann H., 1970. Modelling of Chemical Reactors - Steady state axial heat and mass transfer in tubular reactors numerical investigation of multiplicity. Chemical Engineering Science 25, 187.

131. Vemuri Balakotaiah and Dan Luss, 1991. Explicit runaway criterion for catalytic reactors with transport limitations. AIChE Journal 37 (12), 1780.

132. Papageorgiou J.N., Abello M.C. and Froment G.F., 1994. Kinetic modelling of the catalytic oxidation o-xylene over an industrial $\mathrm{V}_{2} \mathrm{O}_{5}-\mathrm{TiO}_{2}$ (anatase) catalyst. Applied Catalysis A: General 120, 1743. 
Referencias biblicugraficas

133. Anastasov A., 2003. Deactivation of an industrial $\mathrm{V}_{2} \mathrm{O}_{5} / \mathrm{TiO}_{2}$ catalyst for oxidation of o-xylene into phthalic anhydride. Chemical Engineering and Processing 42, 449.

134. Nikolov V.A. and Anastasov A.I.,1992. Pretreatment of a VanadiaTitania catalyst for partial oxidation of 0 -xylene under industrial conditions. Ind. Eng. Chem. Res. 31,80.

135. Demaria F., Longfield J.E. and Butler G., 1961. Catalytic reactor design. Industrial and Engineering Chemistry 53(4), 260.

136. Ahmed M. and Fallen R.W., 1980. Tubular reactor design: two dimensional models. Chemical Engineering Science 35, 889.

137. Van Welsenaere R.J. and Froment G.F., 1970. Parametric sensitivity and runaway in fixed bed catalytic reactors. Chemical Engineering Science 25, 1503.

138. Hunt M.L. and Tien C.L., 1990. Non-Darcian flow, heat and mass transfer in catalytic packed-bed reactors. Chemical Engineering Science $45(1), 55$

139. Daniel O.B., Jorge E.G. and Josh A.P., 1989. Wall-cooled fixedbed reactors: parametric sensitivity as a design criterion. AIChE Journal 35(2), 287.

140. Mcgreavy C. and Adderley C.I., 1973. Generalized criteria for parametric sensitivity and temperatura runaway in catalytic reactors. Chemical Enginrrring Science 28,577 .

141. Schnitzlein K. and Hofmann H., 1987. An alternative model for catalytic fixed bed reactors. Chemical Engineering Science 42 (1), 2569.

142. Suter D., Bartroli A., Schneider F., Rippin D.W.T. and, Newson E.J., 1990. Radial flow reactor optimization for highly exothermic selective oxidation reacitons. Chemical Engineering 45 (8), 2169.

143. Valstart J. M., Van Den Berg P. J. and Oysermans J., 1975. Comparison between two dimensional fixed bed reactor calculations and measurements. Chemical Engineering Science, $30,723$. 
144. Kershenbaum L.S.; L6pez-Isunza F., 1982. Dynamic behavior of an industrial scale fixed-bed reactor, 7th International Symposium on Chemical Reaction Engineering, Boston, USA, 4-/6 October. American Chemical Society-Symposium Series 196, 109.

145. Nikolov V.; Anastasov A.; Elenkov D.; Ganev G.; Dimitrov N., 1988. A new approach to the oxidation of o-xylene into phthalic anhydride in a fixed bed of vanadium-titanium catalyst. Chemical Engineering and Processing 24(3), 157.

146. Kerănen J., Camiti P., Gervasini A., Iiskola E., Auroux A. and Niinisto L., 2004. Preparation by atomic layer deposition and characterization of active sites in nanodispersed vanadia/titania/silica. Catalysis Today 91-92 (15),67.

147. Gervasini A., Carniti P., Kerănen J., Niinistő I. and Auroux A., 2004. Surface characteristics and activity in selective oxidation of o-xylene of supported $\mathrm{V}_{2} \mathrm{O}_{5}$ catalysts prepared by standard impregnation and atomic layer deposition. Catalysis Today $96(4), 187$.

148. Grzybowska-Swierkosz B., 1997. Active centres on vanadia-based catalysts for selective oxidation of hydrocarbons. Applied Catalysis A: General $157(1-2), 409$.

149. Grzybowska-Swierkosz B., 1997. Vanadia-titania catalysts for oxidation of o-xylene and other hydrocarbons. Applied Catalysis A: General $157(1-2), 263$ :

150. Busca G., Centi G. and Trifiro F., 1986. n-Butane selective oxidation on vanadium-based oxides : Dependence on catalyst microstructure. Applied Catalysis (25), Pages 265.

151. Bond G.C., 1997. Preparation and properties of vanadia/titania monolayer catalysts. Applied Catalysis A: General 157(1-2), 91.

152. Bulushev D.A., Kiwi-Minsker L., Zaikovskii V.I., Lapina O. B., Ivanov A.A., Reshetnikov S.I. and Renken A., 2000. Effect of potassium doping on the structural and catalytic properties of V/Ti-oxide in selective toluene oxidation. Applied Catalysis A: General $202(2), 243$. 
Referencias bibliogräicas

153. Nobbenhuis M.G. and Baiker A., 1992. Titania-supported vanadium oxide catalysts for the selective oxidation of oxylene to phthalic anhydride : Influence of vanadia content on activity and surface species. Applied Catalysis A: General $85(2), 157$.

154. Golinelli G. and Trifir6 F., 1994. Selective oxidation of oxylene to phthalic anhydride over the Eurocat $\mathrm{V}_{2} \mathrm{O}_{5} / \mathrm{TiO}_{2}$ catalysts. Catalysis Today $20(1), 153$.

155. Wachs I.E. and Weckhuysen B.M., 1997.structure and reactivity of surface vanadium oxide species on oxide supports. Applied Catalysis A: General $157(1-2), 67$.

156. Grzybowska-\$wierkosz B.. 1994. Decomposition of isopropanol on Eurocat $\mathrm{V}_{2} \mathrm{O}_{5} / \mathrm{TiO}_{2}$ catalysts. Catalysis Today $20(1), 165$.

157. Cristina R.D., Portela M.F. and Bond G.C., 1995. Oxidation of 0xylene to phthalic anhydride over $\mathrm{V}_{2} \mathrm{O}_{5} / \mathrm{TiO}_{2}$ Catalysts.: I. Influence of catalyst composition, preparation method and operating conditions on conversion and product selectivities. Journal of Catalyst 157, 344.

158. Cristina R.D., Portela M.F. and Bond G.C., 1995.Oxidation of oxylene to phthalic anhydride over $\mathrm{V}_{2} \mathrm{O}_{5} / \mathrm{TiO}_{2}$ Catalysts.: IV. Mathematical modelling study and analysis of the reaction network. Journal of Catalyst 164, 276.

159. Bond G.C., 1989. Mechanism of the oxidation of o-xylene to phthalic anhydride. Journal of Catalysis $116(2), 531$.

160. Bond G.C., Zurita J.P. and Paul S.F, Gellings J., Bosch H., Van Ommen J.G. and Kip B.J., 1986. Structure and reactivity of titania-supported oxides. Part 1: vanadium oxide on titania in the sub- and super-monolayer regions. Applied Catalysis 22(2), 361 .

161. Bignardi G., Cavani F., Cortelli C., De Lucia T., Pierelli F., Trifiro F., Mazzoni G., Fumagalli C. and Monti T., 2006. Influence of the oxidation state of vanadium on the reactivity of $V / P / O$, catalyst for the oxidation of $n$-pentane to maleic and phthalic anhydrides. Journal of Molecular Catalysis A: Chemical 
$244(1-2), 244$.

162. Cavani F., Ligi S., Monti T., Pierelli F., Trifiro F., Albonetti S. and Mazzoni G., 2000. Relationship between structural/surface characteristics and reactivity in $n$-butane oxidation to maleic anhydride: The role of $v^{3+}$ species. Catalysis Today $61(1-4)$, Pages 203.

163. Bond G.C., König P., 1982. The vanadium pentoxide-titanium dioxide system: Part 2. Oxidation of o-xylene on a monolayer catalyst. Journal of Catalysis 77(2), 309.

164. Bulushev D.A., Kiwi-Minsker L. and Renken A., 2000. Transient kinetics of toluene partial oxidation over $V / T i$ oxide catalysts. Catalysis Today $61(1,-4), 271$.

165. Centi G., Pinelli D., Trifiro F., Ghoussoub D., Guelton M. and Gengembre L., 1991. Surface structure and reactivity of $V-T i-0$ catalysts prepared by solid-state reaction. 2. Nature of the active phase formed during o-xylene oxidation. Journal of Catalysis $130(1), 238$.

166. Bulushev D.A., Rainone F. and Kiwi-Minsker I., 2004. Partial oxidation of toluene to benzaldehyde and benzoic acid over model vanadia/titania catalysts: role of vanadia species.Catalysis Today 96(4), 195.

167. Bulushev D.A., Kiwi-Minsker L., Rainone F: and Renken A., 2002. Characterization of Surface Vanadia Forms on V/Ti-Oxide Catalyst via Temperature-Programmed Reduction in Hydrogen and Spectroscopic Methods. Journal of Catalysis 205 (1), 115.

168. Bulushev D.A., Reshetnikov S.I., Kiwi-Minsker I. and Renken A., 2001. Deactivation kinetics of $V / T i-o x i d e$ in toluene partial oxidation. Applied Catalysis A: General 220 (1-2), 31.

169. Bulushev D.A., Kiwi-Minsker I., Zaikovskii V.I., Lapina O. B., Ivanov A.A., Reshetnikov S.I. and Renken A., 2000. Effect of potassium doping on the structural and catalytic properties of $V / T i$-oxide in selective toluene oxidation Applied Catalysis A: General 202(2), 243. 
Referencias bibliouráficas

170. Bulushev D.A., Kiwi-Minsker L. and Renken A., 2000. Transient kinetics of toluene partial oxidation over $V / T I$ oxide catalysts. Catalysis Today $61(1-4), 271$.

171. Bulushev D.A., Kiwi-Minsker L., Zaikovskii V.I. and Renten A., 2000. Formation of active sites for selective toluene oxidation during catalyst synthesis via solid-state reaction of $\mathrm{V}_{2} \mathrm{O}_{5}$ with $\mathrm{TiO}_{2}$. Journal of Catalysis $193(1), 145$.

172. Dias C.R., Portela M.F. and Bond G.C., 1997. Symthesis of phthalic anhydride: catalysis, kinetics, and reaction modeling. Catalysis Review- science and Engineering $33(3,4), 319$.

173. Vanice M.A., 2007. An analysis of the Mars-van Krevelen rate expression. Catalysis Today 123, 18.

174. Satterfield C.N. Mass transfer in heterogeneous catalysis. M.I.T. press; England 1970.

175. Hegedus L., Oh S.H and Baron K., 1977. Multiple steady states in an isothermal, integral reactor: the catalytic oxidation of Carbon monoxide over Platinum-Alumina. AIChE Journal 23(5), 632 .

176. Puszynski J. and Hlavacek V., 1980. Experimental study of traveling waves in nonadiabatic fixed bed reactors for the oxidation of carbon monoxide. Chemical Engineering Science 35 , 1769

177. Eigenberger G., 1972. On the dynamic behaviour of the catalytic fixed-bed reactor in the region of multiple steady states- $I$. The influence of heat conduction in two phase models. Chemical Engineering Science 27,1909.

178. Sheintuch M. and Adjaye J., 1990. Excitable waves in a fixed bed reactor: ethylene oxidation on platinum. Chemical Engineering Science $45(7), 1897$.

179. Eigenberger G., 1972. On the dynamic behaviour of the catalytic fixed-bed reactor in the region of multiple steady states. The influence of the boundary conditions in the catalyst phase. Chemical Engineering Science 27, 1917. 
180. Mikus O., Pour V., Halavacek V., 1981. Experimental investigation of temperature fields and multiple steady states in deactivated fixed-bed reactors. Journal of Catalysis 69, 140.

181. Olsen R., Williams W., Song X., Schmidt D., Aris R., 1992. Dynamics of homogeneous-heterogeneous reactors. Chemical Engineering Science 47 (9). 2505.

182. Vortmeyer D. and Schuster J., 1983. Evaluation of steady flow profiles in rectangular and circular packed beds by a variational method. Chemical Engineering Science 38(10), 1691. 


\section{Apéndices}




\section{Apándice $\mathbf{A}$.}

\section{Permeabllidad en un lecho empacado}

En el Capitulo 2, seccion 2.1, se mostraron algunas de las ecuaciones de cantidad de movimiento que se utilizan para predecir los perfiles de velocidad en un lecho empacado con una relación de diámetro de tubo a particula baja. Estas ecuaciones utilizaron términos de permeabilidad que son utilizados para evaluar las resistencias viscosas e inerciales y la calda de presión en un lecho empacado. Estas ecuaciones de permeabilidad se presentan a continuacion.

1. Delmas y Froment [85] $\left(d_{\varepsilon} / d_{p}=6.25\right)$ y Kufner $y$ Hofmann [44] $\left(d_{\varepsilon} / d_{p}\right.$ $=4-12$ ) utilizaron:

$$
\begin{aligned}
& k(\varepsilon)=\frac{1}{-7.53\left(\frac{\varepsilon}{1-\varepsilon}\right)\left[1+4\left(\frac{1-\varepsilon}{\varepsilon^{3}}\right)\right]} \\
& k_{2}(\varepsilon)=\frac{1}{-0.20\left[1+3.55\left(\frac{1-\varepsilon}{\varepsilon^{3}}\right)\right]}
\end{aligned}
$$

2. En la Tabla A.1 se muestran algunos de los estudios que utilizaron las relaciones $A .3$ y A.4.

Tabla A. 1 .

Trabajos que utilizaron las ecuaciones (A.3) y (A.4).

\begin{tabular}{lcc}
\hline Referencia & No. & $d_{c} / d_{p}$ \\
\hline Ergui & {$[73]$} & $>10$ \\
Macdonald et al. & {$[82]$} & $>10$ \\
Daszkowski y Eigenberger & {$[61]$} & 5 \\
Foumeny y Ma & {$[62]$} & $5-20$ \\
Papageorgiuou y Froment, & {$[30]$} & 5.8 \\
Giese et al. & {$[56]$} & 9.3 \\
Winterberg y Tsotsas & {$[54]$} & $4-40$ \\
Winterberg et al.. & {$[66]$} & 5.9 \\
Felice et al. & {$[83]$} & $<10$
\end{tabular}




$$
\begin{aligned}
& k(\varepsilon)=\frac{1}{150\left(\frac{(1-\varepsilon)^{2}}{\varepsilon^{3}}\right)} \\
& k_{2}(\varepsilon)=\frac{1}{1.75\left(\frac{1-\varepsilon}{\varepsilon^{3}}\right)}
\end{aligned}
$$

3.- Bey y Eigenberger [63] $\left(d_{t} / d_{p}=3.3-11\right)$ utilizaron:

$$
\begin{aligned}
& k(\varepsilon)=-\frac{1}{150\left(\frac{(1-\varepsilon)^{2}}{\varepsilon^{2}}\right)} \\
& k_{2}(\varepsilon)=-\frac{1}{1.75\left(\frac{1-\varepsilon}{\varepsilon}\right)}
\end{aligned}
$$

4. Eisfeld y Schnitzlein [60] utilizan para un amplio rango de relaciones $d_{c} / d_{p}$ y flujos de alimentación las siguientes Ecuaciones:

$$
\begin{aligned}
& k(\varepsilon)=-\frac{1}{K \bar{\varepsilon}\left[\frac{\varepsilon(1-\varepsilon)}{\bar{\varepsilon}(1-\bar{\varepsilon})}\right]^{0.25}} \\
& k_{2}(\varepsilon)=-\frac{1}{k(\varepsilon) \frac{1}{6 K}\left(\frac{1.15}{\left(d_{r} / d_{p}\right)^{2}}+0.87\right)^{2}} \\
& K=\frac{154}{36}
\end{aligned}
$$

$d_{p h}=\frac{d p}{6}\left(\frac{\bar{\varepsilon}}{1-\bar{\varepsilon}}\right)\left(1+\frac{2}{3\left(\frac{d_{t}}{d_{p}}\right)(1-\bar{\varepsilon})}\right)^{-1}$ 
5.- Eisfeld [80] y Freund et al., [58], recomiendan las siguientes Ecuaciones:

$k(\varepsilon)=-\frac{1}{A\left(\frac{(1-\varepsilon)^{2}}{\varepsilon^{3}}\right)}$

$k_{2}(\varepsilon)=-\frac{1}{B\left(\frac{1-\varepsilon}{\varepsilon^{3}}\right)}$

$A=163.55 A_{w}^{2}, B=1.2 B_{w}$

$A_{v}=B_{v}=1+\left(\frac{1}{2\left(d_{\varepsilon} / d_{p}\right)(1-\varepsilon)}\right)$

6.-Eisfeld [60], Reichelt et a]. [47] utilizaron:

$k(\varepsilon)=-\frac{1}{1.54 A_{\square}{ }^{2}\left(\frac{(1-\varepsilon)^{2}}{\varepsilon^{3}}\right)}$

$k_{2}(\varepsilon)=-\frac{1}{\frac{A_{w}}{B_{6}}\left(\frac{1-\varepsilon}{\varepsilon^{3}}\right)}$

$A_{-}=\left[1+\left(\frac{2}{3\left(d_{\varepsilon} / d_{p}\right)(1-\varepsilon)}\right)\right]$

$B_{w}=\left(1.15\left[\frac{d_{t}}{d_{p}}\right]^{2}+0.87\right)^{2}$ 


\section{Apéndice B.}

\section{Correlactones para evaluar la fracclón vacia}

Vortmeyer y Schuster [182], para predecir los perfiles de velocidad en un lecho empacado con una relación de $d_{t} / d_{p}$ entre 4-12, utilizaron la siguiente Ecuación para predecir los perfiles de fracción vacfa.

$$
\begin{aligned}
& \varepsilon(r)=\varepsilon_{-}\left(1+C \exp \left[1-\frac{2\left(R_{-}-r\right)}{\phi_{p}}\right]\right) \\
& C=\frac{1-\varepsilon_{-}}{\varepsilon_{-} \exp (1)}
\end{aligned}
$$

La Ecuación de fracción vacía que utiliza Foumeny y Ma [62], para predecir los perfiles de velocidad en un lecho empacado con relaciones de $d_{\varepsilon} / d_{p}$ entre 5-20, es:

$\varepsilon(r)=\varepsilon_{-}\left(1+A \exp \left[-\frac{6\left(R_{\tau}-r\right)}{\phi_{p}}\right]\right)$

$A=\frac{1}{\varepsilon_{\infty}}-1$

Giese et al. [56] $\left(d_{c} / d_{p}=9.3\right)$, Winterberg y Tsotsas [54] $\left(d_{t} / d_{p}=4-40\right)$, winterberg et al. [66] $\left(d_{k} / d_{p}=5.9\right)$, emplearon la siguiente Ecuación para predecir los perfiles de fracción vacía en un lecho empacado:

$\varepsilon(r)=\varepsilon_{-\infty}\left(1+1.36 \exp \left[-\frac{5\left(R_{\tau}-r\right)}{\phi_{b}}\right]\right)$

Kufner y Hoffman $\{44\}$, para predecir los perfiles de velocidad en un lecho empacado con una $d_{t} / d_{p}$ alrededor de 6 , utilizaron la siguiente. Ecuación que describe las oscilaciones y amplitud de la fracción vacía a lo largo del radio del lecho. 
Apéndice B.

Correlaciones para evaluar la fracción vacia

$$
\varepsilon(r)=\varepsilon_{-}+\left[1+\operatorname{cexp}\left[-\left(1+\left(\frac{R_{t}-r}{d_{p}}\right)\right)\right] \cos \left(\left[\frac{R_{e}-r}{d_{p}}\right] 2 \pi\right)\right]
$$

5.-Bey y Eigenberger [63] determinaron los campos de velocidad en un lecho empacado, para $d_{k} / d_{p}$ entre 3.3 y 11 , utilizando la siguiente Ecuacion:

Perfil de fracción vacia en la región cerca de la. pared:

$$
\varepsilon_{u}(r)=0.24+0.76\left(r^{\prime}\right)^{2} ; r^{\prime}<0
$$

(B.7)

Perfil de fracción vacía en el centro del lecho:

$$
\varepsilon_{e}(r)=\varepsilon_{-}+\left(\varepsilon_{-}-0.24\right) \exp \left[-\frac{r^{\prime}}{10}\right]\left(\cos \left[\frac{\pi}{0.876} r^{\prime}\right]\right) ; \quad r^{\prime}>0
$$

Donde :

$$
\begin{aligned}
& I^{\prime}=\frac{\left(\frac{d}{2}-r\right)}{x_{\operatorname{man}}}-1 \\
& x_{\min }=0.5\left(d_{t}-\sqrt{\left(d_{c}-d_{p}\right)^{2}-d_{p}^{2}}\right. \\
& \varepsilon_{-}=0.375+0.34 \cdot \frac{d_{p}}{d_{c}}
\end{aligned}
$$

201 
Apéndice $C$.

Predicción de los perfiles de veloctdad empleando CFD

En este Apéndice se muestra una comparación entre dos métodos para predecir los perfiles de velocidad en un lecho empacado: 1) el métodos aproximado utilizado de manera fundamental en ingeniería, es decir, aquél que acopla la Ecuación de Navier-Stokes con los términos de Darcy y Forchheimer en la ecuación de Ergun, considerando un medio pseudocontinuo $y$ que es utilizado en esta tesis; 2) aquél que describe el sistema puntualmente, empleando las ecuaciones de Navier-stokes en un volumen de control, y que describe al lecho empacado de acuerdo con la geometría del sólido, construyendo una malla computacional que define las dos fases en contacto: Esto se conoce como Dinámica de Fluidos Computacional, CFD.

En el Capitulo 2 se mostró que la predicción de los perfiles de velocidad utilizando las ecuaciones de Navier-Stokes y acoplando los términos de Darcy y Forchheimer, son una forma eficiente de predecir los perfiles de velocidad en los lechos empacados de estudio, y otros que presentan una relación de $d_{t} / d_{p}$ baja $[27,60,76]$. La máxima virtud de este método es el tiempo de cómputo corto. Sin embargo, algunas de las desventajas fueron: el no considerar dentro de la correlación de fracción vacía, su funcionalidad con la posición tangencial y axial; y no utilizar un modelo hidrodinámico que considere la turbulencia en el lecho (ej. el modelo $x-\varepsilon$ ).

Por otro lado, CFD es un método que actualmente es muy popular en la predicción de los campos de velocidad en las diferentes ramas de la ingeniería química. Una de las ventajas de este metodo es que hace posible resolver numéricamente los balances de cantidad de movimiento, energía $y$ materia, en sistemas con geometrias complejas. Sin embargo dos de las principales desventajas son el tiempo de cómputo (muy largo) Y el problema de convergencia en la solución numérica al considerar una representación exacta del sistema. Por esto, se consideran algunas 
idealizaciones (espacios que no existen físicamente entre sరlido-sరlido y pared-sólido) $[39,40,64,65,68]$. Para describir los campos de velocidad el método de CFD aplica las ecuaciones de Navier-stokes sobre un pequeño volumen de control, considerando una geometría dentro del lecho. El tamaño y número de volúmenes de control, a lo que se le llama densidad de la malla, es normalmente determinado por el usuario, y afecta directamente en la exactitud de la solución. Esta malla describe la topología del sistema en 3D.

En esta tesis, no se intenta hacer un estudio detallado de un paquete de computación de CFD, ya que éste sería otro tema de investigación. Sin embargo, es interesente comparar los resultados que arroja este sofisticado método, con aquéllos obtenidos con la suposición del pseudo-continuo. Para predecir los perfiles de velocidad con CFD se utilizó el paquete FLUENT 6.2. Las consideraciones para la simulación del lecho empacado son:

1. Se consideran los efectos de turbulencia, por lo que el modelo $\mathrm{k}-\varepsilon$ se utiliza para predecir los campos de velocidad. La mayor restricción del modelo $k-\varepsilon$ es que debe utilizarse para un flujo totalmente turbulento, en donde los efectos de la viscosidad molecular son despreciables.

2. Se consideró un lecho empacado con esferas de catalizador. Se tomó al aire como el gas que pasa a través de este lecho. El flujo que se utilizó fue el de la corrida 4 (ver la Tabla 2.2), el cual corresponde a un $R e_{p}$ igual a 320 , es decir, un régimen de flujo turbulento.

3. La configuración del empaque es aquélla que se muestra en la Figura c.1. Esta no considera el contacto entre sólido-sólido y sólidopared del lecho, ya que cuando esto se consideró la simulación del modelo no converge. 
Las Ecuaciones y consideraciones sobre el modelo $k-\varepsilon$, que utiliza CFD, se pueden revisar en la referencia [68] y en la guía del usuario del FLUENT 6.2 .

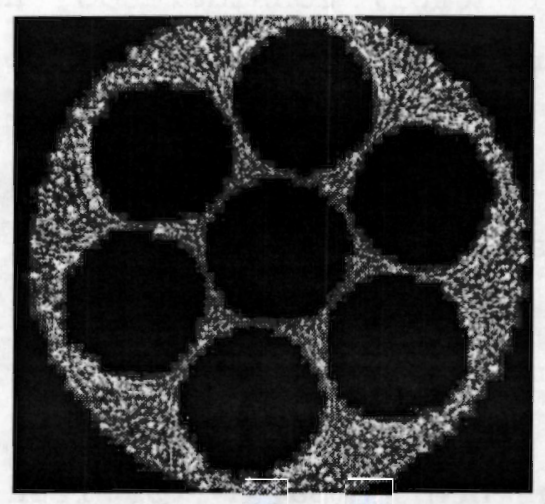

Figura C.1. Configuración del empaque en CFD. Vista frontal (FLUENT 6.21 .

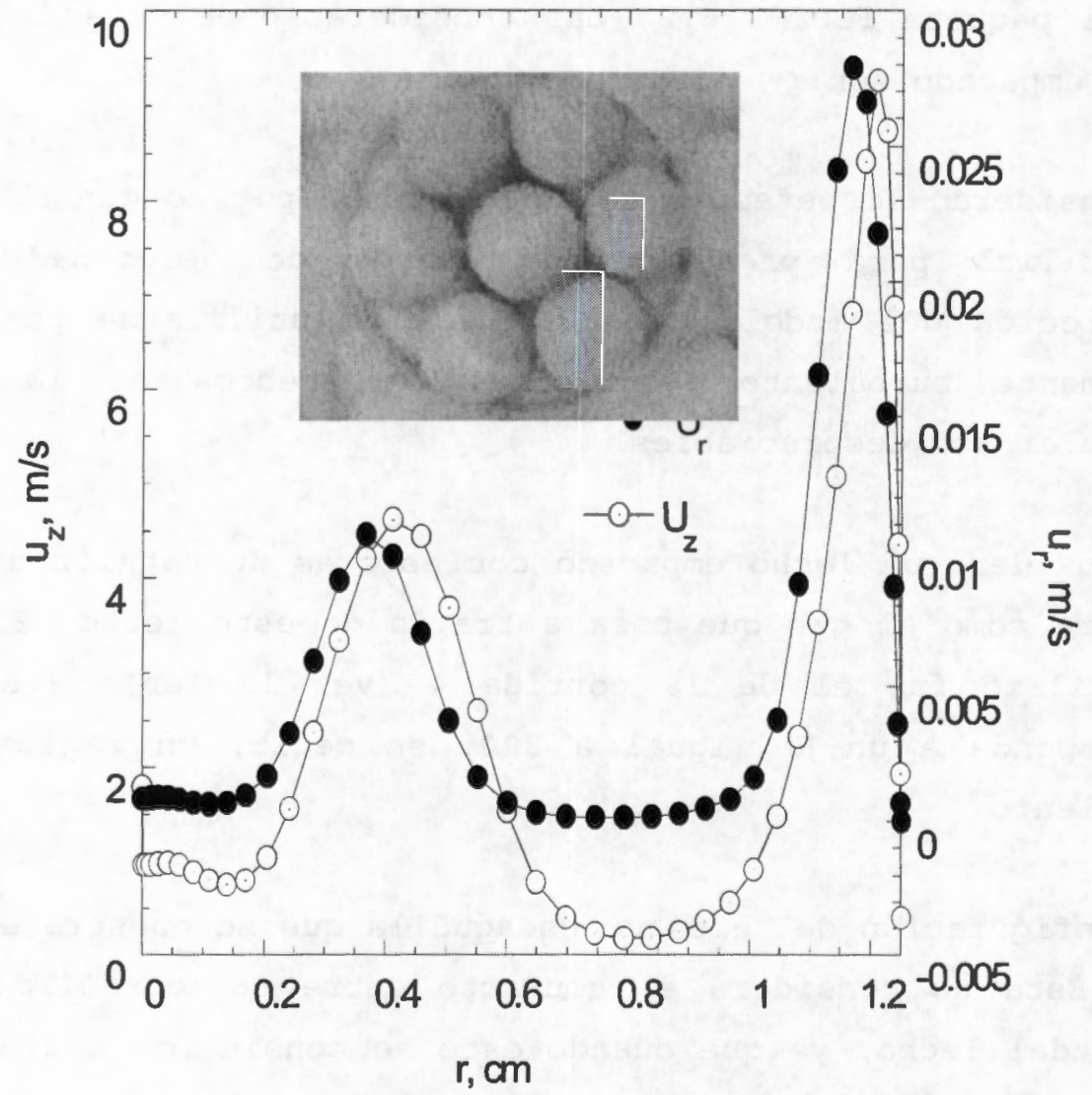

Figura C.2. Perfiles de velocidad, empleando el modelo 2-F, para un $\operatorname{Re}_{\mathrm{p}}=315$. 
La Figura C.2 presenta los perfiles de velocidad dimensional, tanto de la componente axial como radial, predichos con el modelo 2-F. Las Figuras C. 3 a C.5 presentan las predicciones de las componentes de velocidad axial, radial y tangencial, respectivamente, al utilizar CFD; el tiempo de cómputo fue alrededor de 3 días. Estos resultados se comparan con los mostrados en la Figura C.2, y se discuten a continuación. La Figura C.3 muestra los perfiles de velocidad (de la componente axial). Los máximos perfiles de velocidad se encuentran en la región cerca de la pared del lecho y a una posición de diámetro de partícula. Los patrones de velocidad muestran variaciones en la posición axial y tangencial en forma periódica, y no constante como en el caso de las predicciones del modelo 2-F. Éste es el resultado de considerar, con CFD, la distribución radial, axial y tangencial de las esferas de catalizador. La velocidad en la región cercana a la pared del lecho fue de alrededor de $11 \mathrm{~m} / \mathrm{s}$ en donde se tenía la presencia de una esfera de catalizador, y alrededor de $8 \mathrm{~m} / \mathrm{s}$ en donde no se tenía ésta. Mientras que a una posición de un diámetro de partícula se tenían velocidades alrededor de $5 \mathrm{~m} / \mathrm{s}$. Las diferencias entre la máxima velocidad predicha, con el modelo 2-F y con CFD, no es mayor a un $20 \%$. La comparación de ambas predicciones señalan que tales diferencias se pueden minorizar si se hacen algunas modificaciones al modelo 2-F. Las principales serían considerar las variación de la fracción vacía en las tres dimensiones y los fenómenos de turbulencia lej. utilizar un modelo $k-\varepsilon$ ). La principal ventaja de hacer esto, y no utilizar CFD, es tener un tiempo de cómputo menor y una mejor descripción de los perfiles de velocidad en el lecho empacado. Esto tendría un impacto directo en los perfiles de temperatura y concentración predichos en el reactor. La Figura C.4 muestra los perfiles de velocidad de la componente de velocidad radial. Las máximas velocidad se encuentra en región cerca de la pared del tubo y a una posición de un diámetro de particula, tendiendo el mismo comportamiento que las predicciones del modelo 2-F, sin embargo, la magnitud para la simulación con CFD está en el rango de 3-5 m/s, a diferencia del modelo $2-F$ en el cual son despreciables. La componente de la velocidad radial muestra también variaciones en la 
posición axial y tangencial. Lo anterior indica que para que el modelo 2-F prediga resultados similares a los de una simulación con CFD, el gradiente de presión radial no debe ser cero.
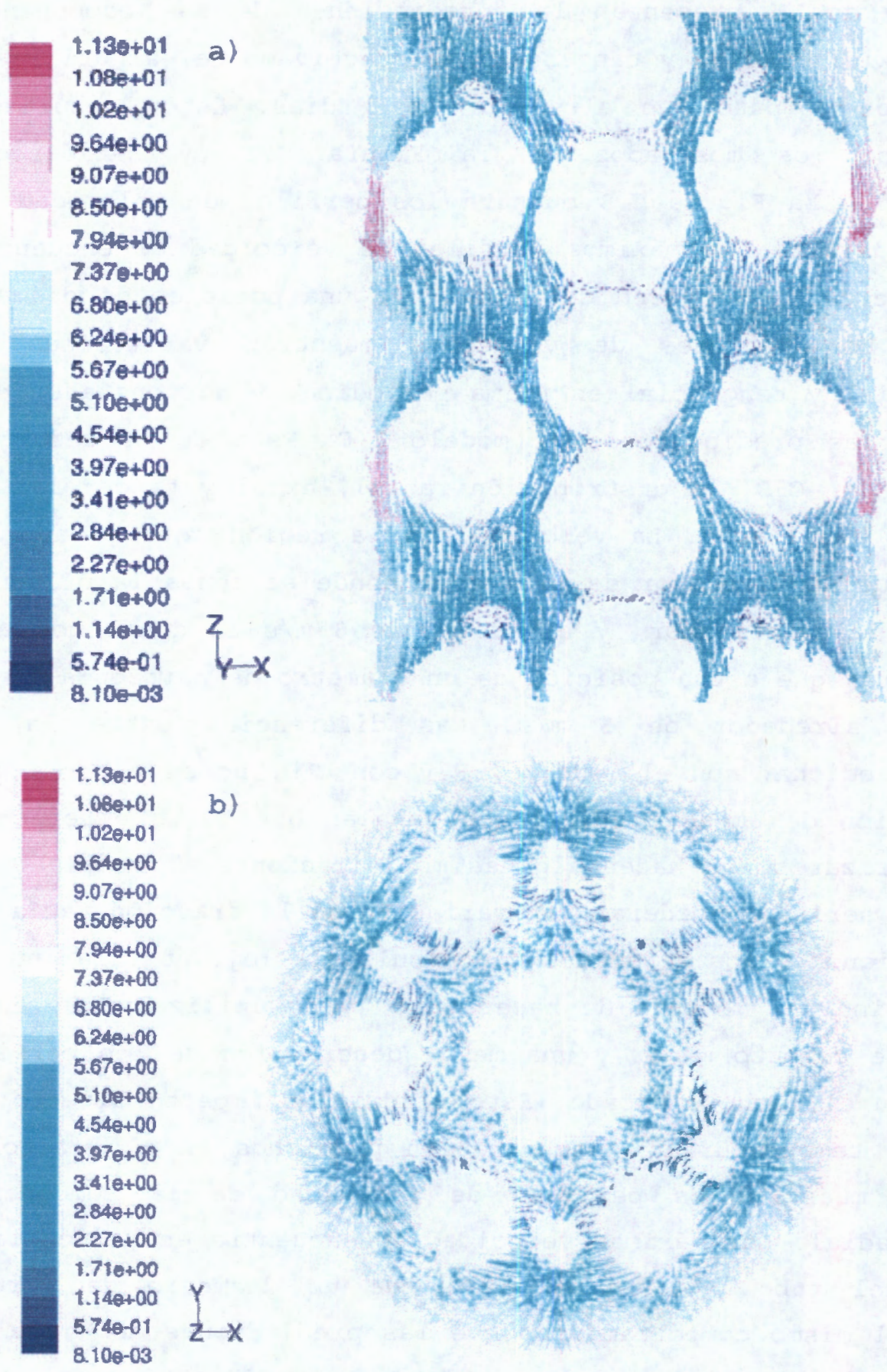

Figura C.3. Perfiles de la velocidad axial. a) Vista lateral y b) vista frontal. 
$4.36 \mathrm{e}+00$

$3.96 \mathrm{e}+00$

$3.570+\infty 0$

$3.170+00$

$2.77 \mathrm{e}+00$

$2.370+00$

$1.97 \mathrm{e}+00$

$1.57 \mathrm{e}+0.0$

$1.18 \mathrm{e}+00$

$7.780-01$

$3.800-01$

$-1.820-02$

$-460-01$

- 8. 15e-01

$-121 e+00$

$-1.61 \theta+00$

$-2.01 \theta+00$

$-2.41 e+00$

$-2.81 e+00$

$-3.20 e+00$

$-3.600+00$

a)

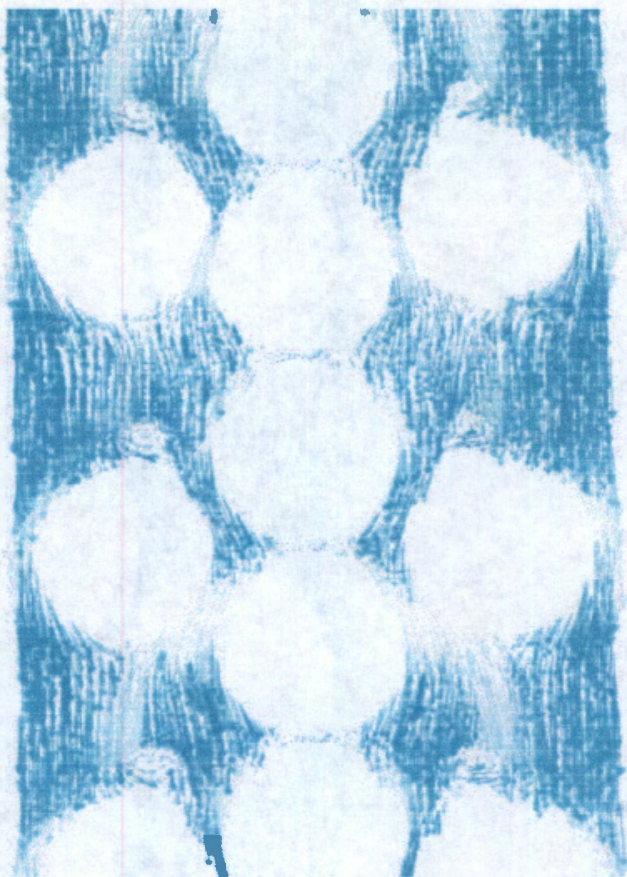

$43 E 0+C 0$

s.serco

$z$

$3.570+10$

3.172+100

277000

estonim

$1.97 \mathrm{n}+10$

$1.512+00$

$1.180+10$

$7 . \operatorname{man} 1$

s.meti

$182 \div$

4 1E 5

$+160-61$

-12 aith

$18+40$

$201+C 0$

Etitico

-2 BI $u$ CLO

$729+30$

$200+30$

b)

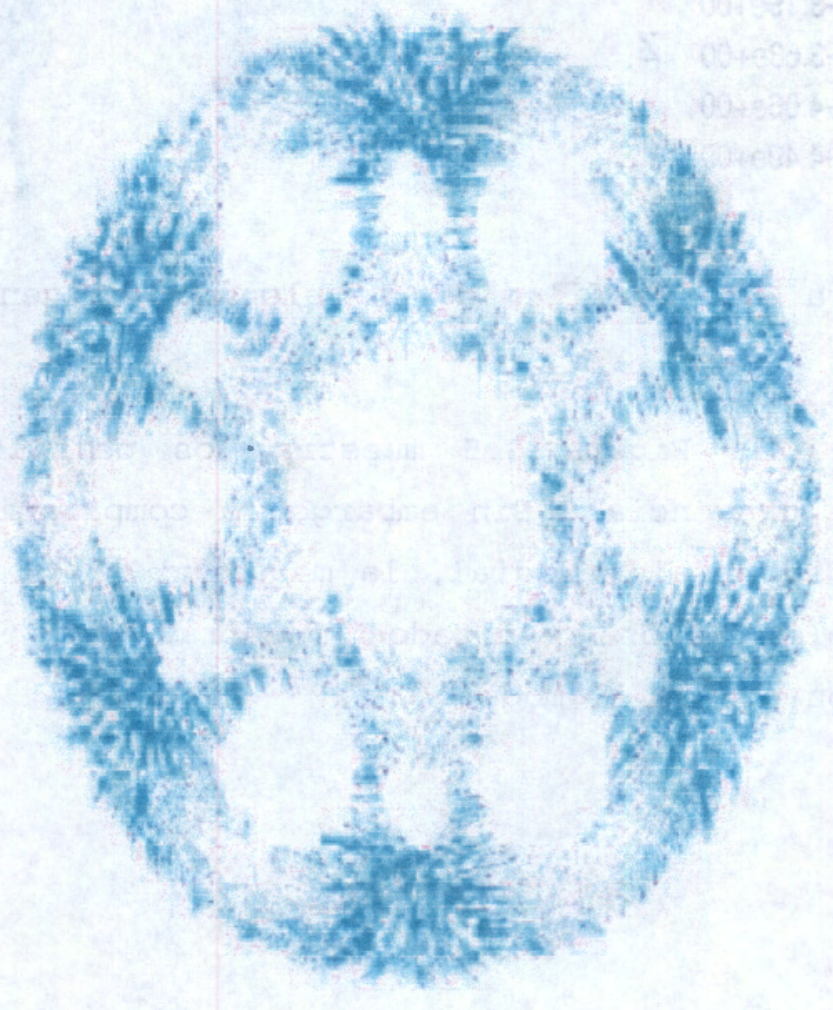

Figura C.4. Perfiles de la velocidad radial. a) vista lateral y b) vista frontal. 


$\begin{aligned} & 4.16 e+00 \\ & 3.73 e+00 \\ & 3.30 e+00 \\ & 2.86 e+00 \\ & 2.43 e+00 \\ & 2.00 e+00 \\ & 1.57 e+00 \\ & 1.13 e+00 \\ & 7.00 e-01 \\ & 2.68 e-01 \\ & -1.65 e-01 \\ & -5.98 e-01 \\ & -1.03 e+00 \\ & -1.46 e+00 \\ & -1.90 e+00 \\ & -2.33 e+00 \\ & 2.76 e+00 \\ & -3.19 e+00 \\ & -3.63 e+00 \quad Z \\ & -4.06 e+00 \\ & -4.49 e+00\end{aligned} \quad Y-X$

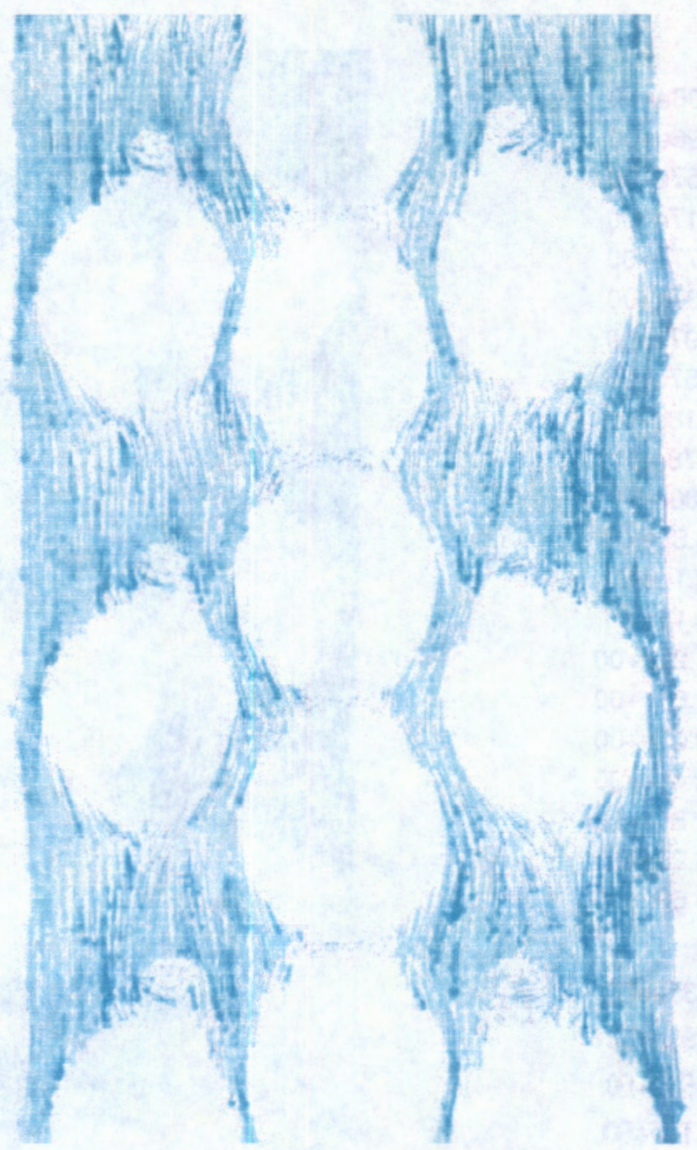

Figura C.5. Perfiles de la velocidad tangencial. a) Vista lateral.

Pinalmente, la Figura C.5 muestra los perfiles de velocidad de la componente tangencial. Sin embargo, a comparación de la componente de la velocidad axial y radial. Ia magnitud de la velocidad tangencial es despreciable. Estos resultados muestran que fue correcto despreciar esta componente en el modelo $2-F$. 
Apöndice D.

Efecto de 10 perfiles de Iracción vacia en la predicción de los campos de velocidad

Los perfiles de fracción vacia juegan un papel importante en la predicción de los perfiles de velocidad en un lecho empacado. Ya que éstos se relacionan con la magnitud de las resistencias inerciales y viscosas en el sistema estudiado.

En las Figuras D.1 Y D.2 se muestran los perfiles de velocidad determinados al utilizar tres distintas correlaciones a diferentes flujos $\left(\operatorname{Re}_{\mathrm{p}} 25\right.$, y 785). Físicamente en el sistema se tienen dos zonas de alta fracción vacia, cerca de la pared del tubo y a una posición de un diámetro de particula. Cuando se utilizo la correlación de Foumeny y Ma [62], ésta sólo es capaz de predecir el comportamiento de los perfiles de velocidad cerca de la pared del tubo. Después de ésta, el perfil de velocidad es constante. Lo anterior muestra que, aquellos trabajos que emplean correlaciones de este tipo para sistema con relaciones $d e d_{c} / d_{p}$ menores a 10, trabajan con incertidumbres que darian lugar a una inadecuada descripción de los perfiles de velocidad.

Cuando se utilizan las correlaciones de Bey .Y Eigenberger [63] y de Klerk [59] se predice un perfil de velocidad con dos máximos a lo largo de la posición radial del lecho. Cerca de la pared y a una posición de un diámetro de particula. Las predicciones cerca de la pared del tubo fueron similares, diferencias de 5 al $20 \%$ dependiendo del flujo de alimentación, sin embargo, a una distancia de un diámetro de partícula de la pared, las diferencias llegan a ser mayores a un 100\%. En la literatura se debe estar consciente de estas diferencias, ya que, una mala elección de una correlación que prediga los perfiles de fracción vacia, se reflejaría en una mala descripción de los perfiles de velocidad $y$ por consiguiente de temperatura $y$ concentración. 

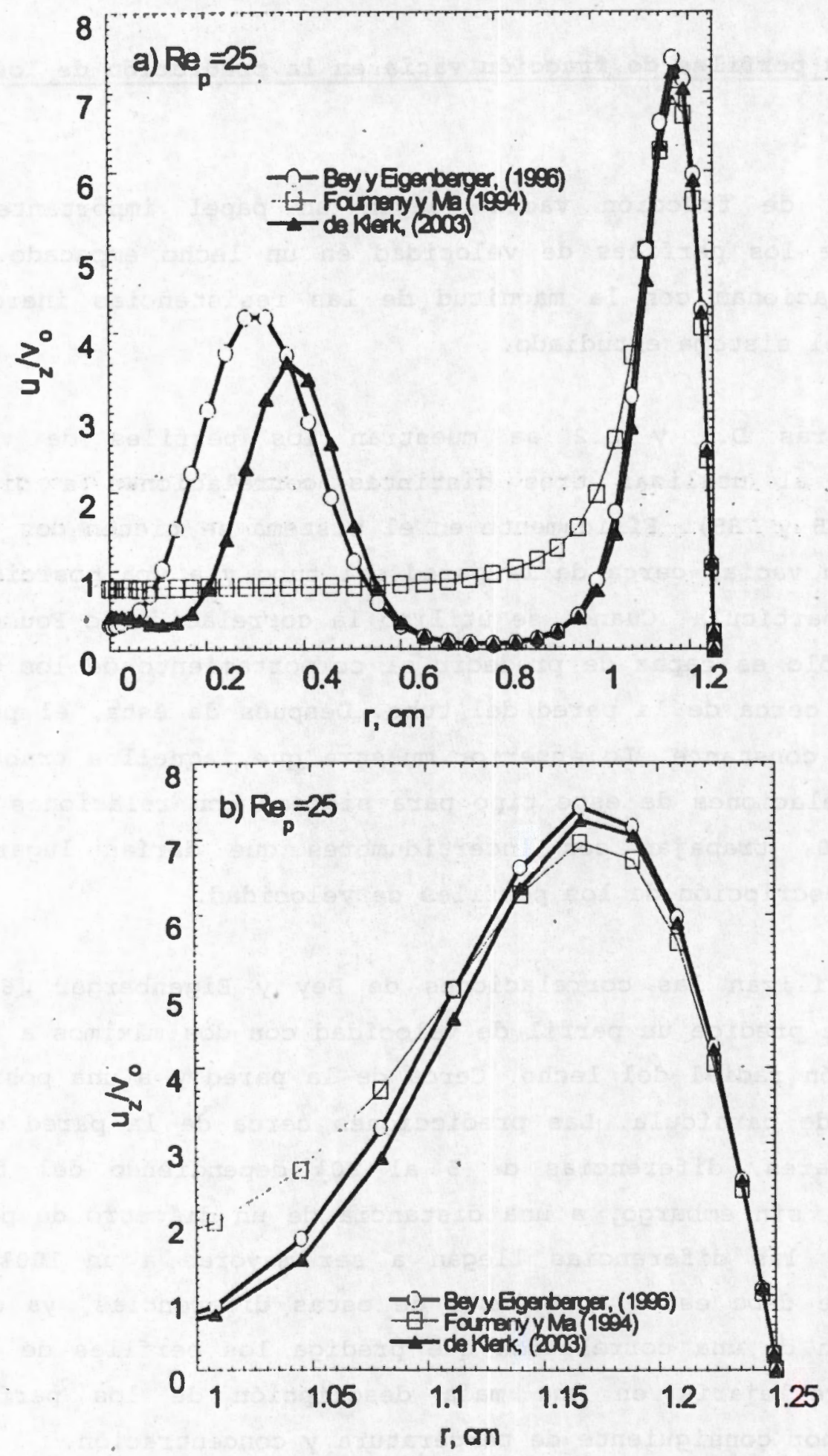

Figura D.1. Efecto de la correlación de fracción vacía utilizada en la predicción de los perfiles de velocidad: a) a lo largo del radio y b) a una distancia de $2.5 \mathrm{~mm}$ de la pared del tubo. 

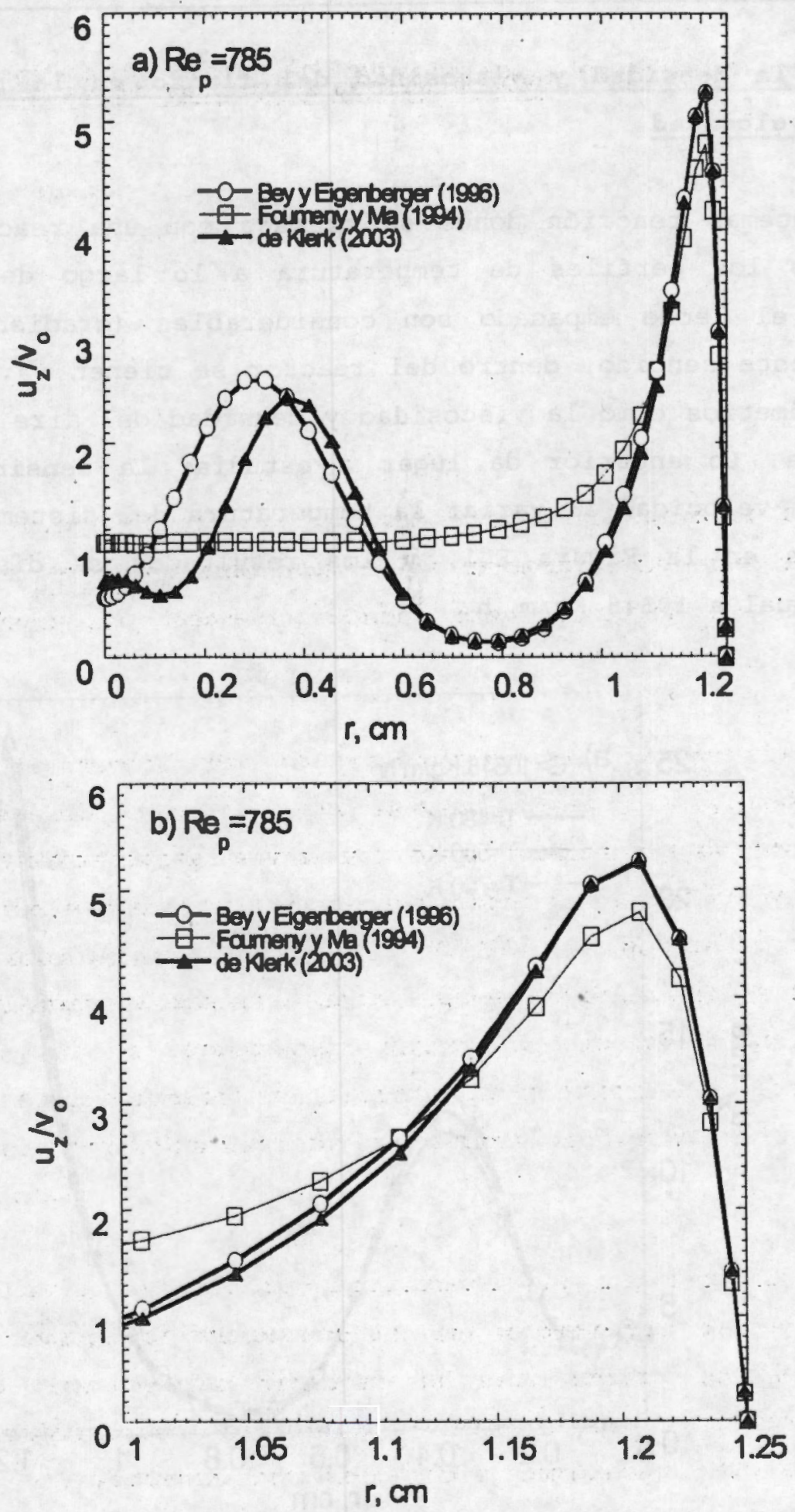

Figura D.2. a) Predicción de los perfiles de velocidad al utilizar diferentes Ecuaciones de fracción vacia: a) a lo largo del radio y b) a una distancia de $2.5 \mathrm{~mm}$ de la pared del tubo. 
Apéndice $\mathrm{E}$.

Efecto de la densidad y viscosidad del fluido en la predicctón del perfil de velocidad

En los sistemas reacción donde se trabaja con una reacción altamente exotérmicas los perfiles de temperatura a lo largo del eje axial $y$ radial en el lecho empacado son considerables (gradientes mayores a $\left.100^{\circ}\right)$. En este sentido, dentro del reactor se tienen variaciones en un 30 en parámetros como la viscosidad y densidad del aire en el interior del sistema. Lo anterior da lugar a estudiar la sensibilidad de los perfiles de velocidad al variar la temperatura del sistema. Este efecto se presenta en la Figura E.1, y los resultados se discuten para un flujo G, igual a $10548 \mathrm{~kg} / \mathrm{m}_{2} \mathrm{~h}$.

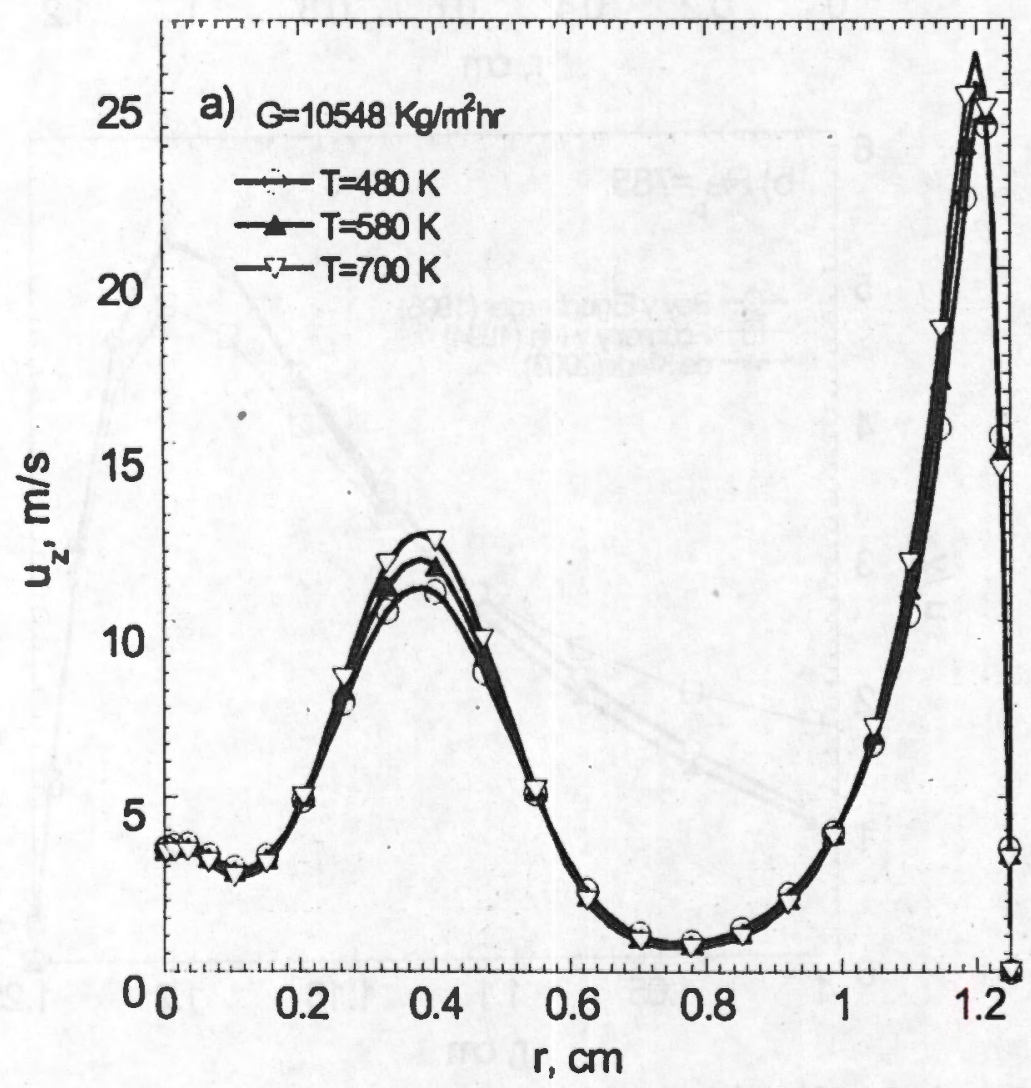

Figura E.1 Efecto de la temperatura en los perfiles de velocidad. 

predicción del perfil de velocidad

Un incremento de la temperatura, provoca un incremento en la viscosidad del gas y una disminución en la densidad del fluido teniendo un incremento en la viscosidad cinemática. Las Ecuaciones de movimiento muestran que la viscosidad afecta al término donde interviene el gradiente de presión, y la viscosidad cinemática al término inercial debido a Forchheimer. En la Figura E.I se observa que las diferencias en la velocidad, a diferentes temperaturas, son mayores en las regiones de alta fracción vacia, no siendo mayores a un 10\%. Por consecuencia un incremento en la temperatura provoca que el gradiente de presión junto con las resistencias inerciales disminuya, lo cual provoca que tal efecto balanceé la magnitud de los perfiles de velocidad en el interior del lecho. Los perfile de velocidad para otras velocidades de alimentación mostraron un comportamiento similar. 


\section{Apéndice E. Dinámica de los perfiles de veloctdad}

En las Figuras F.1 y F.2 se presenta la dinámica de los perfiles de velocidad, para dos $\mathrm{Re}_{\mathrm{p}}, 25$ y 785, desde que se empieza a alimentar fluido hasta que se alcanza el estado estacionario.

Los perfiles de velocidad, para cualquier flujo de alimentación, alcanzan el estado estacionario en aproximadamente 2 segundos (F.1a y F.2a). Por consiguiente cuando se tiene reacción los fenómenos de transporte de calor y masa, asi como la capacitancia de masa y calor, principalmente del solido, son dominantes para alcanzar el estado estacionario en el sistema. Por otro lado, al incrementar la velocidad de alimentación la amplitud de los perfiles de velocidad es menor, lo cual podría tener una relación con el espesor de capa limite, el cual debe ser menor a flujos altos, teniendo asi el efecto observado. Asimismo, la comparación, entre los perfiles de velocidad obtenidos a diferentes $R e_{p}$, mostro que las resistencias viscosas e inerciales son importantes a flujos bajos y altos. 

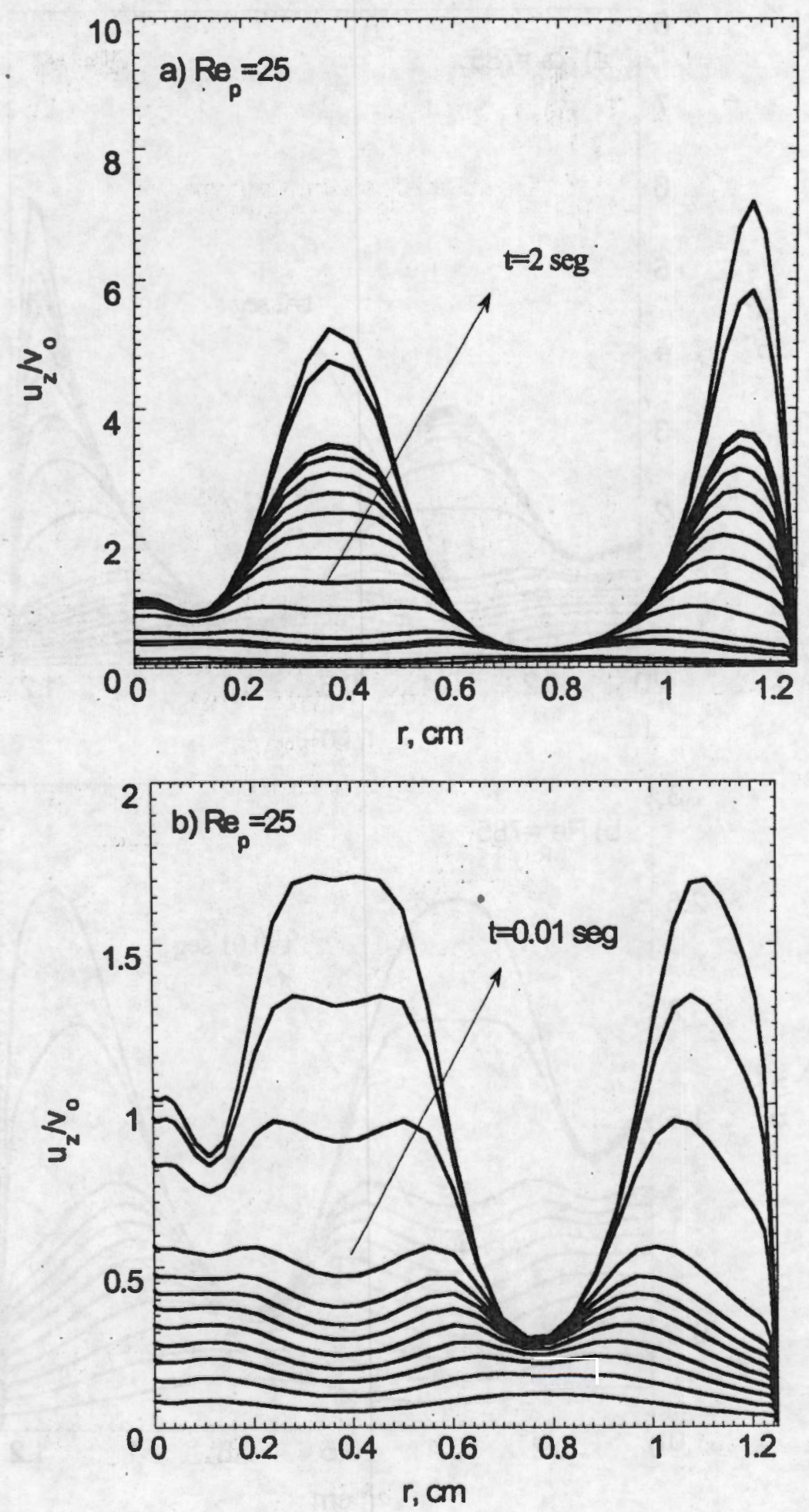

Figura F.1. Comportamiento dinámico de los perfiles de velocidad a un $\mathrm{Re}_{\mathrm{p}}=25$. 

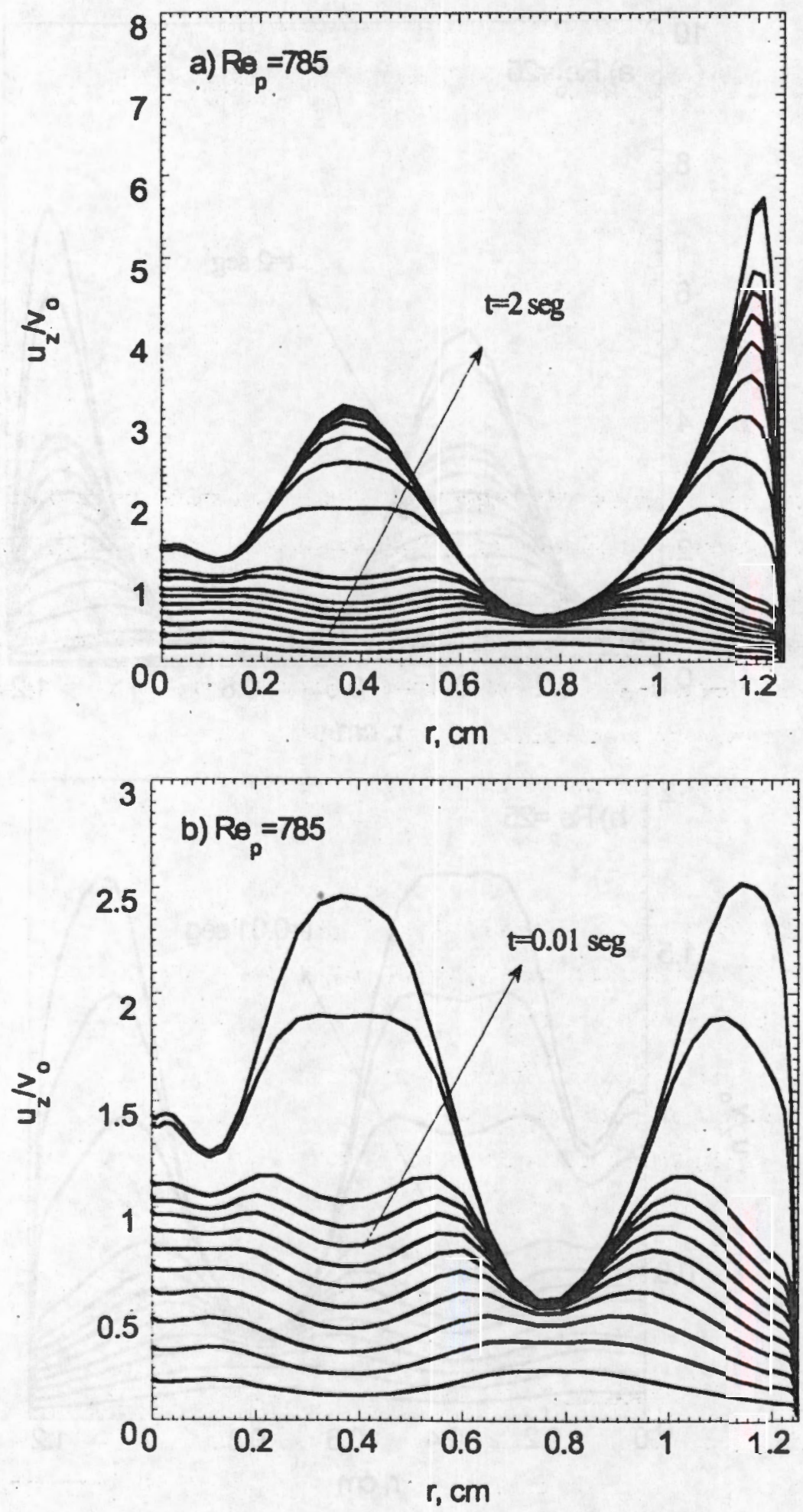

Figura F.2. Comportamiento dinámico de los perfiles de velocidad a un $\operatorname{Re}_{\mathrm{p}}=785$. 
Apéndice G.

Impacto de I perfil de velocidad en la prediccioth del perfil de temperatura

Apéndice $G$.

Impacto del perfil de velocidad en la predicctón del perfil de temperatura

En la Figura G.1 se presenta una comparación de los perfiles de temperatura bajo dos casos, en el primero se considera flujo pistón en el lecho $y$ en el segundo se considera a los perfiles de velocidad. En ambas situaciones se utilizaron los parámetros de transporte de calor estimados para el caso 1-B. El considerar los campos de velocidad provoca que existan dos zonas, cerca de la pared y a una distancia de un diámetro de partícula, en donde la velocidad intersticial llega a ser hasta cinco veces mayor que la velocidad superficial (ver capitulo 2). Estas diferencias provocan que los perfiles de temperatura, varien de 5 a $25^{\circ}$ dependiendo de la posición axial, con respecto al considerar un flujo pistón. Si bien, las diferencias son significantes, como se muestra en las Figuras 3.14-3.21, ambas formas de tratar el problema pueden ajustar los perfiles de temperatura, sin embargo, los parámetros de transporte de calor, $h_{w} y k_{\text {er }}$, son diferentes. In la Figura G.2, se presentan las preediciones de los perfiles de temperatura radiales, cuando se emplean distintas correlaciones para describir la fraccion vacia en el lecho. En esta Figura se observa que, para las distintas correlaciones de fracción vacia utilizadas, las diferencias en los perfiles de temperatura son de 1 a $5^{\circ}$ a la entrada del lecho, siendo mayores cerca de la pared, en donde la velocidad intersticial es mayor. Conforme se avanza en la posición axial del lecho, las diferencias en los perfiles de temperatura incrementan $\left(5-30^{\circ}\right)$, tendiendo las mayores diferencias en la zona cerca de la pared. Esta simulacionn, muestra la sensibilidad de los perfiles de temperatura a los perfiles de fracción vacia. Por esto, una mala correlación de fracción vacía afectaría la estimación de los parámetros de transporte de calor, los cuales afectan sensiblemente los perfiles de temperatura y concentración en el sistema de reacción de estudio. Por lo que, se recomienda que cuando se trabaje con sistemas con bajas relaciones de $d_{t} / d_{p}$, se consideren todas estas situaciones. 


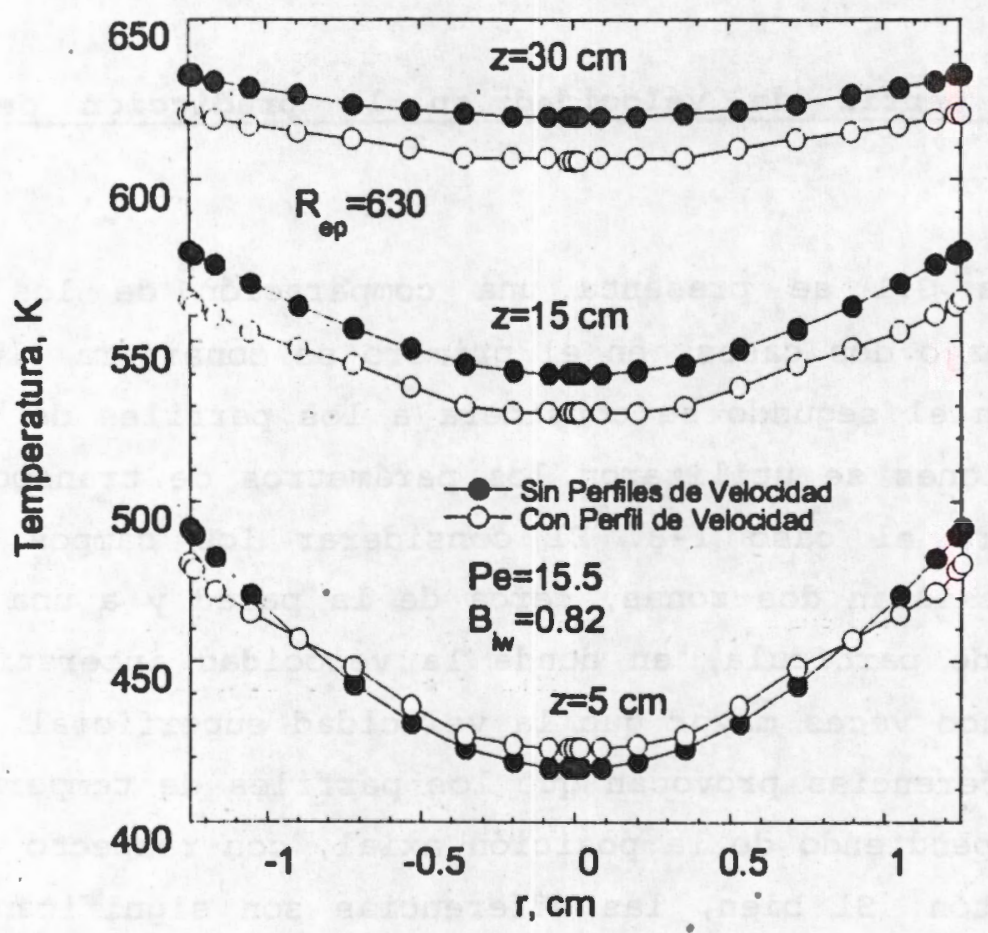

Figura G.1. Efecto de los perfiles de velocidad en la predicción de los perfiles de temperatura.

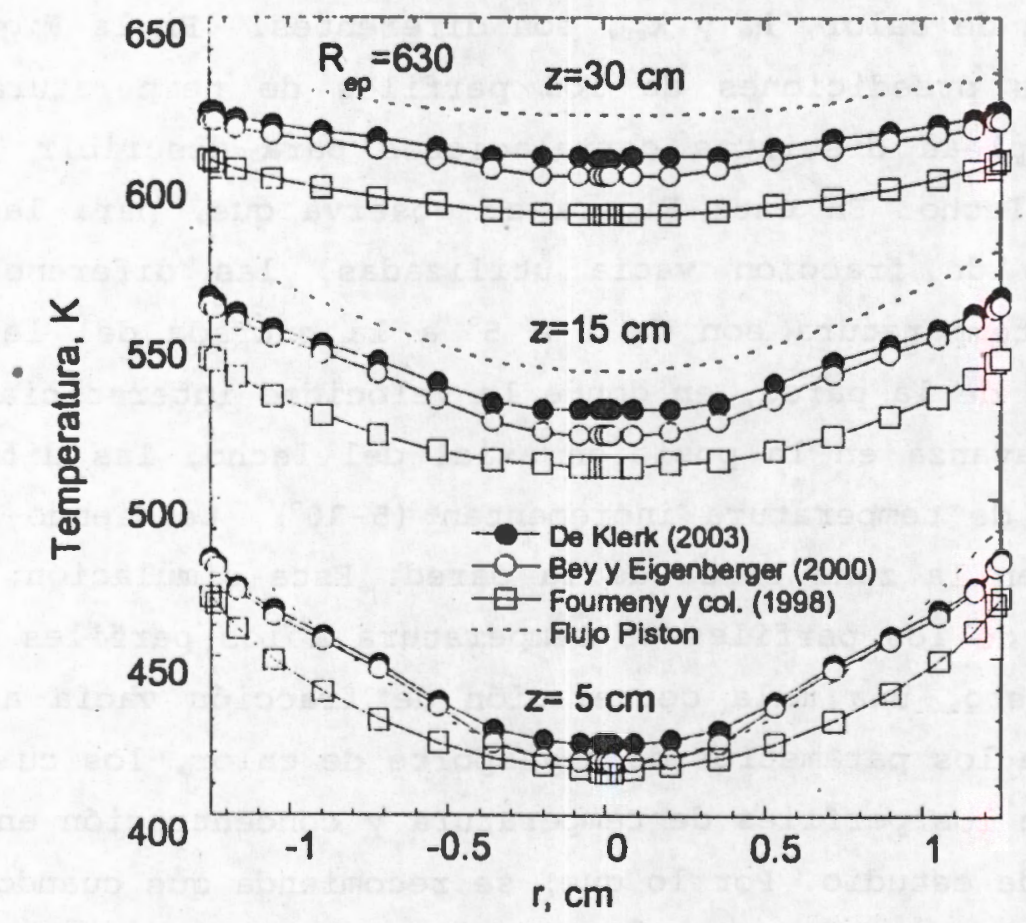

Figura G.2. Efecto de los perfiles de fracción vacía en la prédicción de los perfiles de temperatura. 
Apéndice $\mathrm{H}$.

Evaluación de algunos modelos cinéticos

Apéndice H.

Evaluación de algunos modelon cinéticos

Los modelos cinéticos propuestos por Calderbank et al. (1977) [8], Skrzypek et al. (1985) [17] y Papageorgiou et al. (1994) [132], son aquellos que han predicho $y$ explicado, con relativo éxito, el comportamiento de la reacción de la oxidación parcial de ox a AF; al emplear un catalizador, concentraciones $y$ temperaturas cercanas a las utilizadas en la industria. Cada uno con sus ventajas y desventajas [14-16-18,19]. En esta sección se compara la predicción de cada uno de los modelos cinético, con el objetivo de mostrar la bondad de cada uno de estos modelos en la predicción de las observaciones experimentales, del reactor industrial. Las Figuras H.1 y H.2 presentan la comparación de los perfiles de temperatura y concentración predichos con estos tres modelos cinéticos, sin considerar la desactivación del catalizador. Aqui se emplean el modelo del reactor pseudo-homogéneo en una dimensión (HOM-1D) en conjunto con las condiciones de operación correspondientes al Exp-1 (ver Tabla 4.2).

La Figura H.l muestra la comparación de los perfiles de temperatura, al emplear el coeficiente de transporte de calor (U) estimado de estudios sin reacción (ver capitulo III). Los resultados muestran que cuando se emplea el modelo cinético de Calderbank et al. la magnitud del punto caliente es similar a la experimental, a diferencia de los otros modelos cineticos. Para predecir los perfiles experimentales de temperatura con los modelos cinéticos de Skrzypek et al. y Papageorgiou et al. es necesario reajustar los calores de reacción, lo cual seria erróneo.

Los tres modelos cinéticos predicen una conversión cercana al 100 y y un rendimiento de salida de AF cercano al experimental ( 0.8). Sin embargo, los perfiles de concentración a lo largo del reactor son diferentes para cada modelo cinetico. El modelo cinético de Calderbank et al. [8] es el único que predice el comportamiento observado de los perfiles de concentración; en la posición del punto caliente el ox ha 
Apéndice $\mathrm{H}$.

Evaluación de algunos modelos cinéticos

reaccionado en un $70 \%$ Y el AF se ha producido en un 20\%, además los compuestos de oxidación total se han formado casi totalmente $y$ el oT muestra ser el primer intermediario [11] (ver Figura H.2).

Debido a lo anterior, en el capitulo 4 se emplea el modelo cinetico propuesto por Calderbank et al. para estudiar el efecto de las diferentes variables que intervienen en el modelamiento del reactor. Asi también el Capitulo 5 se presenta otra alternativa para considerar dentro del modelo cinético la desactivación del catalizador.

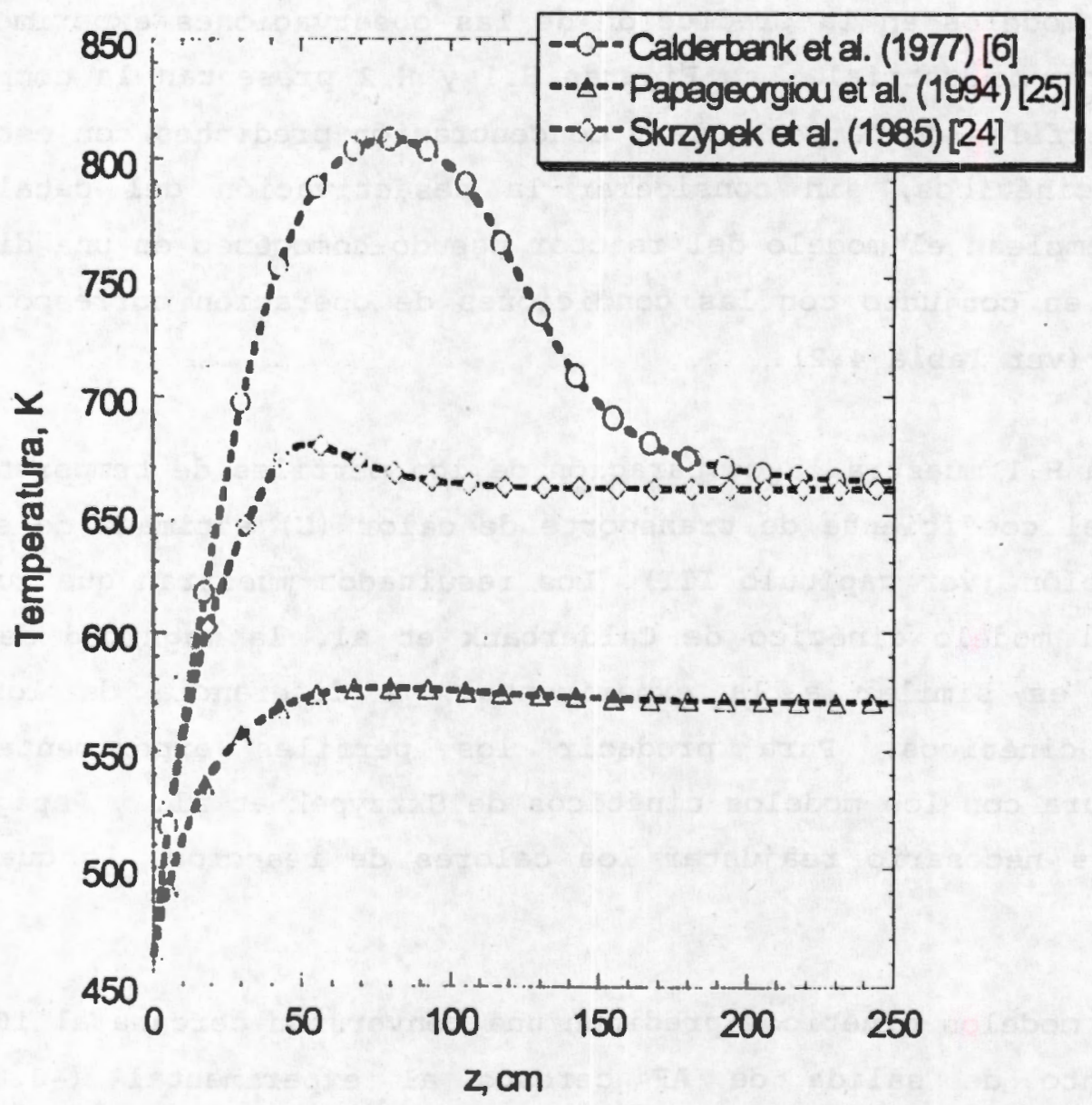

Figura H.1. Comparación de los perfiles de temperatura para predecir el comportamiento del Exp-1 empleando diferentes modelos cinéticos. 

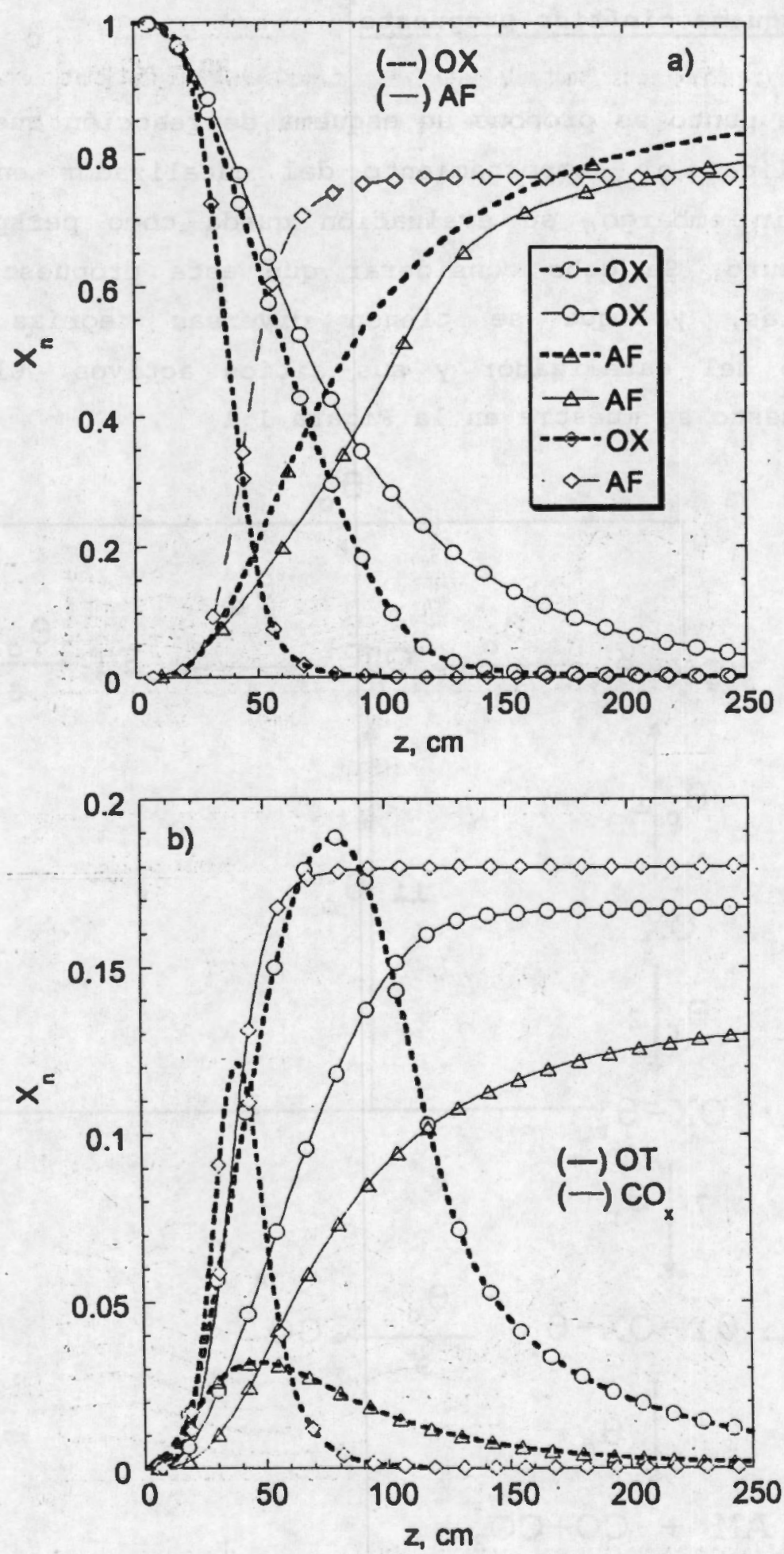

Figura H.2. Comparación de los perfiles de concentración, para predecir el comportamiento del Exp-1 empleando diferentes modelos cinéticos: a) AF y OT; b) OT y $\mathrm{CO}_{x}$ 


\section{Apéndice I. Esquema cinático propuesto}

En el presente punto se propone un esquema de reaccion que pudiera ser capaz de explicar el comportamiento del catalizador en un reactor industrial; sin embargo, su evaluación queda como perspectiva a un trabajo a futuro. Se debe considerar que esta propuesta es una de muchas posibles, ya que se tienen diversas teorías acerca del comportamiento del catalizador y sus sitios activos. El esquema de reacción propuesto se muestra en la Figura I.1

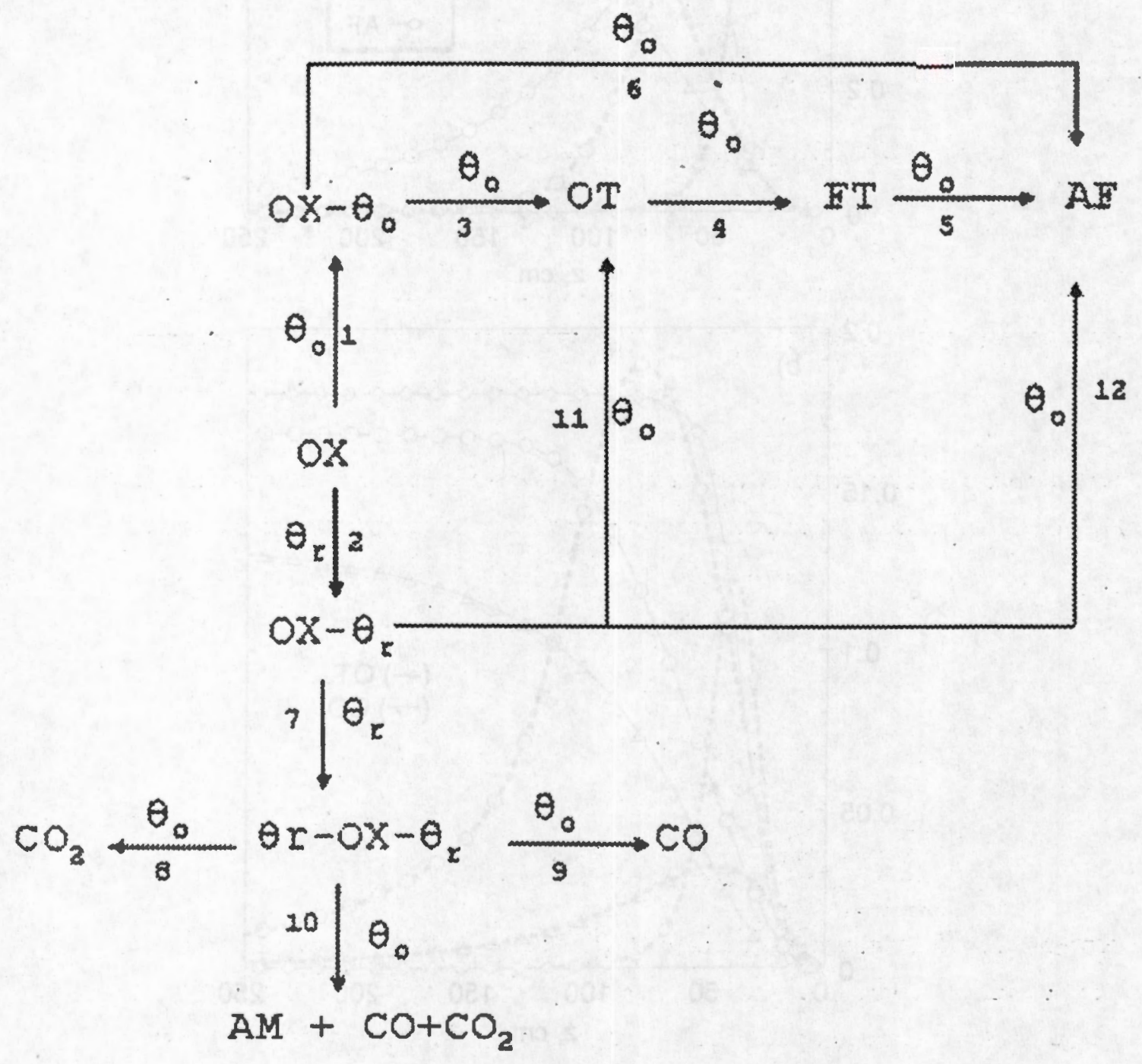

Figura I.1. El esquema cinético propuesto 
El esquema cinético mostrado considera los siguientes puntos:

a) El catalizador industrial contienen aproximadamente una monocapa de vanadio externamente depositado en la titania [168]. El vanadio de la monocapa es el responsable de la actividad catalitica [11].

b) Los estudios de espectroscopia de Raman muestran que la superficie del catalizador contienen dos especies activas de vanadio: monovanadatos $y$ polivanadatos $[11,149,157]$. La especie $V=0$ parece ser el sitio activo para llevar a cabo la oxidación parcial de los hidrocarburos.

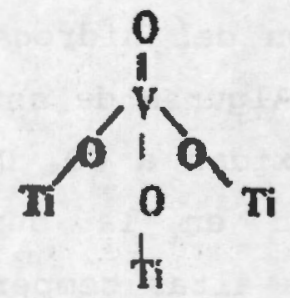

Monovanadatos, $\theta_{0}$<smiles>CCO[V](=O)O[V](C)=O</smiles>

Polivanadatos, $\theta_{0}$

c) Los sitios responsables de la oxidación total y de la adsorciónpolimerización $y$ deshidrogenación del hidrocarburo, son ácidos fuertes Lewis los cuales pueden corresponder a vanadio que se encuentran en un estado de oxidación bajo $\left(\mathrm{v}^{+3}\right)$ y al titanio debajo de la monocapa, debido a defectos estructurales del vanadio disperso en la monocapa [11].

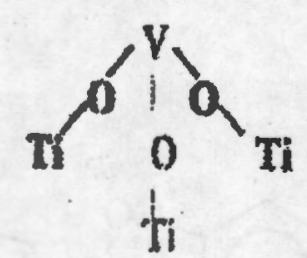<smiles>CON(O)O[Tl]</smiles><smiles>O[GaH]O</smiles>

Monovanadato, $\theta_{r}$ Polivanadato, $\theta_{r}$ Titanaia, $\theta_{r}$

Sin embargo, se debe considerar la presencia de radicales $-\mathrm{OH}$, tanto en las especies de vanadio como en la titania, las cuales pueden actuar como ácidos de tipo Bronsted que contribuyen como sitios responsables 
de la oxidación total o la fuerte adsorción del hidrocarburo, principalmente de ox [163].

d) Los sitios reducidos se regeneran con el oxígeno que proviene de dos fuentes: a) el oxígeno molecular que se incorpora con la mezcla de reacción; 2) el oxígeno que proviene de la estructura del catalizador a través de un proceso de difusión. La re-oxidación de los sitios reducidos de vanadio, depende básicamente de la concentración de ox $y$ el perfil de temperatura a lo largo del lecho. Diversos trabajos $[15,19]$ muestran que la regeneración del sitio es muy rápida. Sin embargo, debido a las altas concentraciones y tiempos cortos de residencia en el reactor, la posibilidad de tener una fuerte adsorción del hidrocarburo impide la re-oxidación del sitio reducido [11]. Algunas de estas moléculas se quedan en la superficie y otras se oxidan a $\mathrm{CO}_{x}$. Después de la reacción, las moléculas que se quedan en la superficie del catalizador se oxidan con aire ( $\sin O X)$ a altas temperaturas $y$ por largos tiempos para recuperar la actividad del catalizador. Si éste no se trata a mayores temperaturas y tiempos, conforme incrementa el tiempo de operación del reactor (meses o años), se estaría contribuyendo gradualmente a la desactivación irreversible.

e) Los estudios experimentales $y$ de caracterización en la superficie del catalizador en un reactor industrial $y$ en uno de escala laboratorio han mostrado que durante la reacción de oxidación parcial de OX, principalmente algunos subproductos son [11,14$16,163]$ :<smiles>CC1=CC=C=CC1C</smiles><smiles>Cc1ccccc1C=O</smiles><smiles>O=C1OCc2ccccc21</smiles><smiles></smiles><smiles>C1=C[GeH]OO1</smiles>

o-xileno o-tolualdehido Ftalida

(FT)

A. Ftálico

A. Maléico (OX) (OT)

(AF) (AM) 
Además de éstos se ha detectado $\mathrm{CO}, \mathrm{CO}_{2}$ y $\mathrm{H}_{2} \mathrm{O}[14,16]$. Asl como moleculas de hidrocarburo polimerizadas en la superficie del catalizador $[11,19]$, las cuales presentan diferentes grados de deshidrogenación lo que hace dificil su oxidación total de la superficie del catalizador. Lo anterior hace necesario considerar este fenómeno dentro el modelo cinético.

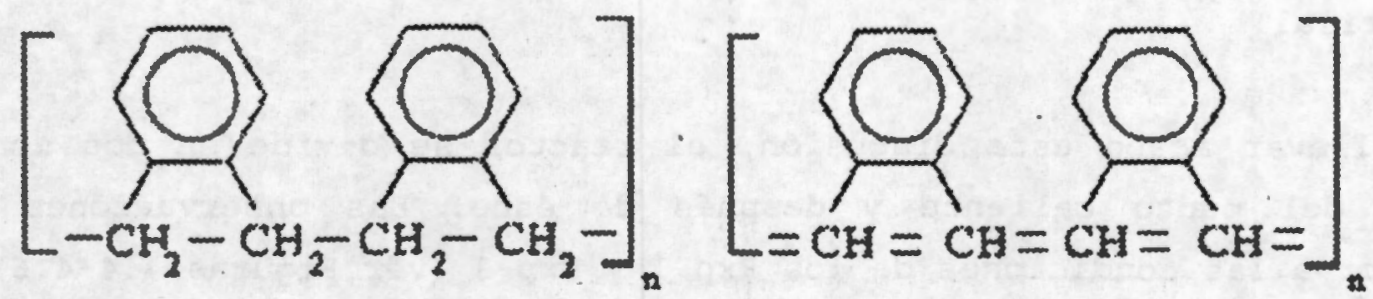

Moléculas polimerizas en la superficie, normalmente $\mathrm{Ox}$.

Durante el mecanismo de reacción, la adsorción de ox en el vanadio es el paso más dificil de la reacción, ya que las consecuentes reacciones se llevan a cabo sin dificultad [159].

La regeneración de los sitios presentes durante la reacción es crucial para llevar a cabo la oxidación parcial, por lo que ésta podría considerarse asi:

$2 \theta_{\mathrm{r}}+\mathrm{O}_{2} \longleftrightarrow 2 \theta_{0}$

$\theta_{x}+\mathrm{O}_{2} \longleftrightarrow \theta_{0}+\mathrm{O}^{*}$

$0^{*}+\theta_{\mathrm{r}} \longleftrightarrow \theta_{0}$

El esquema cinético en la Figura I.1 se planteó con el propósito de predecir el comportamiento experimental del reactor industrial tanto en estado estacionario como en el transitorio. De acuerdo a las observaciones en el reactor, cada una de estas reacciones tendrá preferencia a la oxidación total, parcial o hacia una fuerte adsorción, 
dependiendo la temperatura y concentración de ox dentro del lecho. Lo anterior señala la necesidad de verificar este modelo cinético en un reactor de laboratorio dentro de intervalos de temperaturas, concentraciones y tiempos de residencia, con la idea de simular las condiciones de experimentación que se tienen a lo largo del reactor industrial. Para entender mejor esto, se intenta relacionar el esquema cinético propuesto con el comportamiento observado en el reactor industrial.

Para llevar acabo esta discusión, el reactor se divide en dos zonas: antes del punto caliente $y$ después de éste. Las observaciones del reactor a las condiciones de los Exp-1 y Exp-3 (ver Figuras 4.4-4.6) se toman como referencia para hacer esta discusión. Hasta la posición del punto caliente el ox se ha convertido alrededor de un 80 mientras que la producción de AF es marginal (ver la Figura 4.5). Las referencias $[15,16]$ muestran que en esta zona del reactor se tiene la mayor producción de $\mathrm{OT}, \mathrm{CO}, \mathrm{CO}_{2}$ y $\mathrm{AM}$, asi como el comienzo de la formación de FT (ver la Figura I.1). Lo anterior sugiere que ésta es una zona rica en sitios selectivos a la oxidación total de ox como consecuencia de la alta concentración de éste en esta región del reactor. Este paso de reacción se muestra con el paso 7 , el cual es esencial para lograr los pasos 8-10. En esta zona del reactor no se tiene la oxidación total de AF debido a su alta estabilidad a la oxidación total [163]. Además que la. aparente alta concentración de sitios reducidos se refleja en una baja producción de AF.

En la otra zona del reactor, después del punto caliente, se tiene la mayor produccion de AF (ver la Figura 4.5). Esto sugiere que la baja concentración de ox ocasiona que se tenga una zona rica en sitios oxidados y los pasos 1,3-6, ver la Figura I.1, tienen lugar. La alta concentración de sitios selectivos a la oxidación parcial sugiere que la producción de AF vía el paso estequiométrico 6 es mayor que en la zona localizada antes del punto caliente. 
Finalmente, la regeneración del catalizador (Ecuaciones J.1-J.3), que denotan la re-oxidación del catalizador, tienen lugar en ambas zonas del reactor. Una mayor concentración de ox sugiere una fuerte adsorción de estas moléculas impidiendo el paso del oxígeno molecular para la regeneración de estog sitios. Una mayor temperatura sugiere que la superficie se regenere [132], ya que se promueve la oxidación total de las moléculas fuertemente adsorbidas. 
Apéndice J.

Efecto de los paránetros de transporte de calor y maga en 108 perfiles de tomperatura y concentración

En este Apéndice se hace un estudio de sensibilidad de los perfiles de temperatura y concentración a cambios en los parámetros de transporte de calor y masa. Esta sección se divide en dos partes. En la primera se muestra el efecto de cambios en los parámetros de transporte de calor y masa efectivos: $h_{w}, k_{e r}, k_{a z}, D_{e x}, D_{a z}$ en términos del numero adimensional que los contiene, $B_{1 w}, P_{e r}, P_{e z}, P_{\text {emr }} y P_{e m s}$, respectivamente. En la segunda se presenta el efecto de cambios en los parámetros $k_{g} y$ $h_{g}$ que describen el transporte de masa y calor en la interfase solidogas.

\section{J.1 Parámetros de transporte de calor efectivos, $h_{\text {, }} k_{\text {etr }} k_{\text {es }}$ y Dor}

Las Figuras J.1-J.3 muestran la sensibilidad del perfil de temperatura y concentración axial al cambiar el valor del número de Peclet de masa axial $P_{\text {emz }}$ (ver la Figura J.1.a), del número de peclet de calor axial $P_{\text {ehz }}$ (ver la Figura J.1.b), del numero de Biot $B_{\text {iw }}$ (Figura J.2.a), del número de Peclet de calor radial modificado Pohr (Figura J.2.b), y del número de Peclet de masa radial modificado $P_{\text {emr }}$ (Figura J.3.a). Estas simulaciones utilizan las condiciones de operación del Exp-1 y el modelo HOM-2D. La desactivación del catalizador se considero con la Ecuación (4.28). Los resultados muestran lo siguiente:

1) Variaciones en los parámetros $P_{\mathrm{ema}} Y P_{\mathrm{emr}}$ no ocasionan variaciones significantes en los perfiles de temperatura $y$ concentración en el reactor (ver las Figuras J.1 Y J.3).

2) La variación de los parámetros de transporte de calor $h_{w}, k_{a z} y k_{e r}$, muestra una alta sensibilidad en los perfiles de temperatura $y$ concentración en el reactor. 

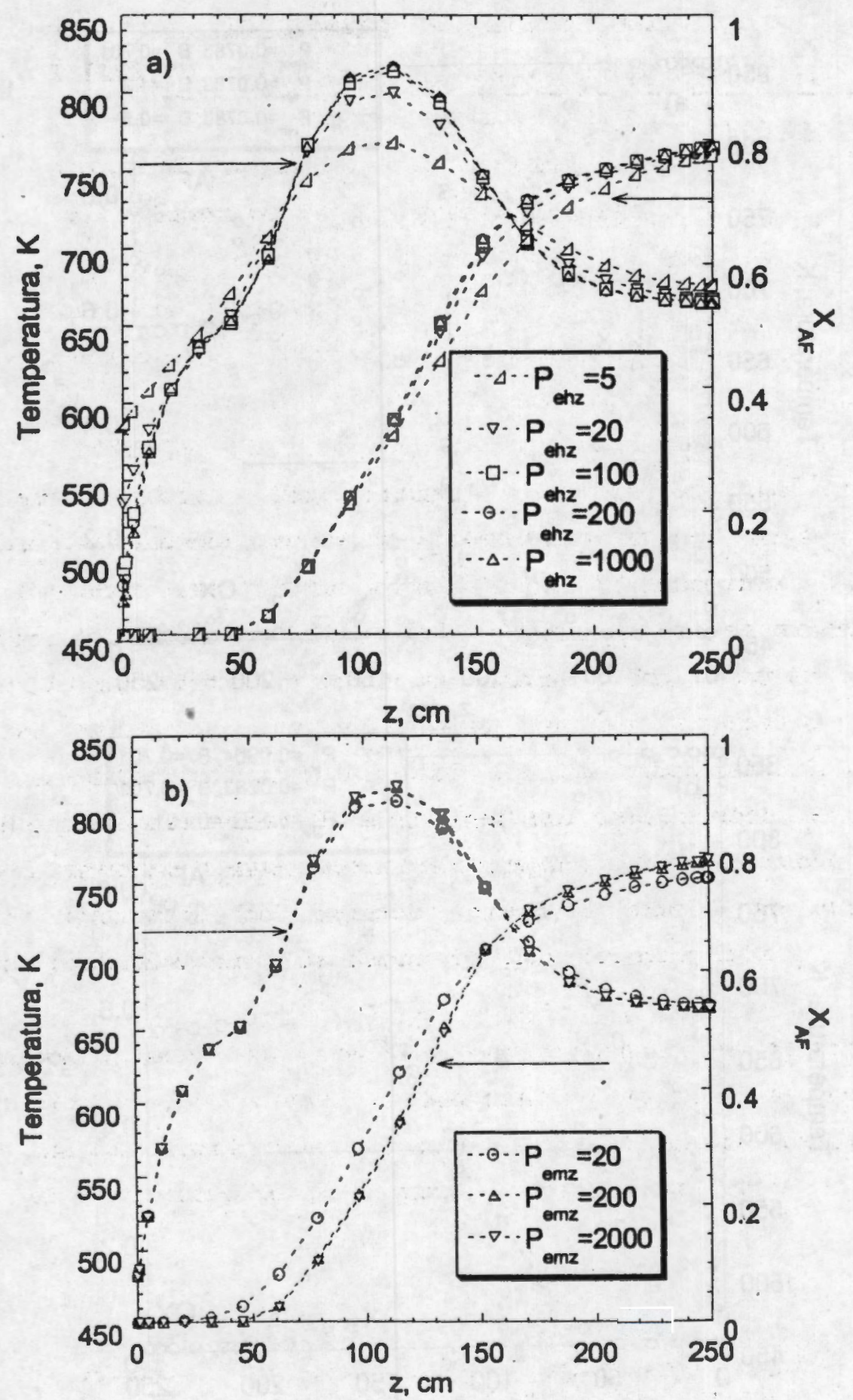

Figura J.1. Efecto de los fenómenos de dispersion axial en la predicción de los perfiles de temperatura, y concentración de AF:

a) $P_{\text {ehz }} Y$ b) $P_{\text {enzz }}$. 


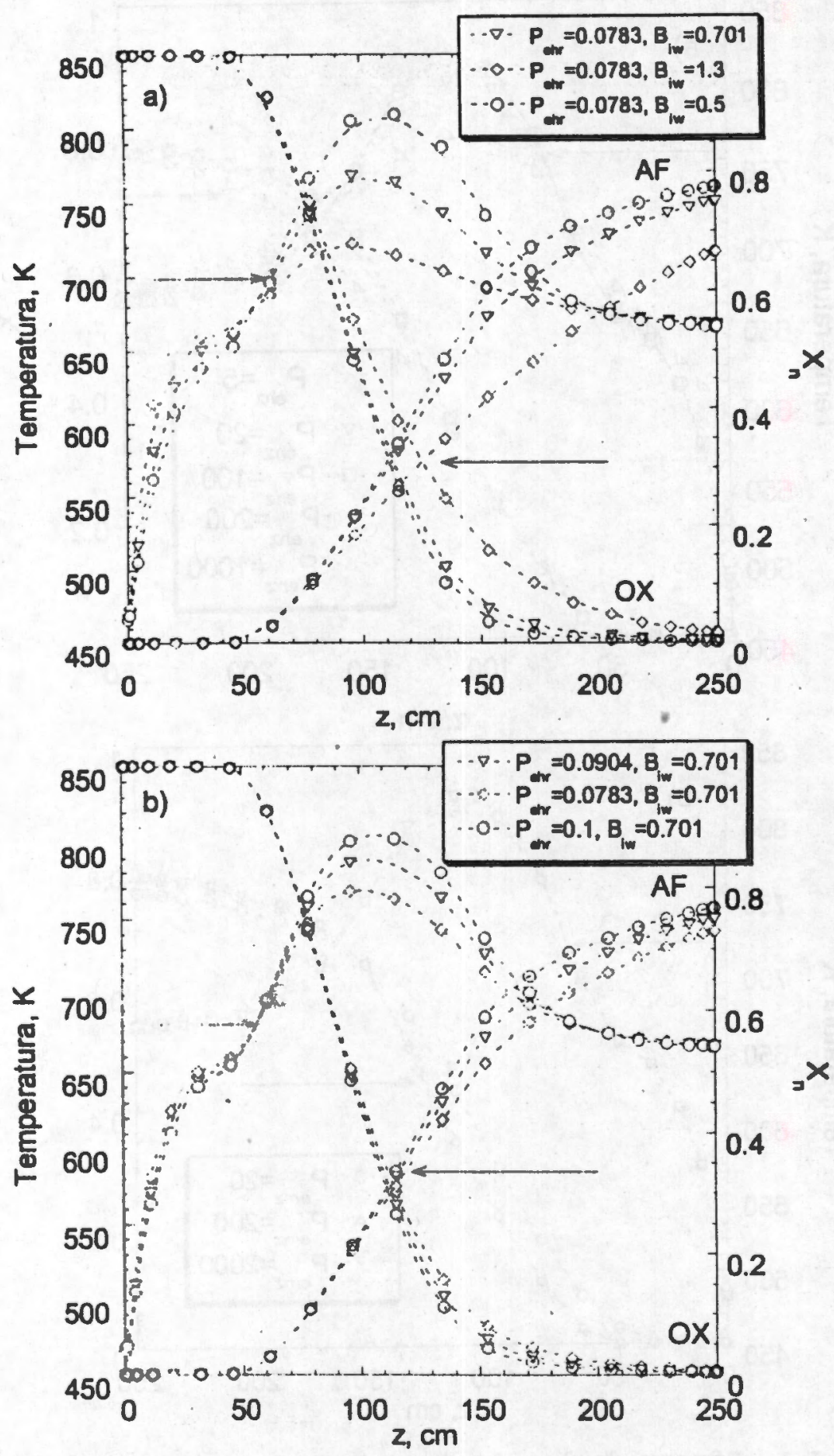

Figura J.2. Efecto de los parámetros de transporte de calor en los perfiles de temperatura $y$ concentración: a) efecto del $B_{i w}, y$ b) efecto del Pehr 


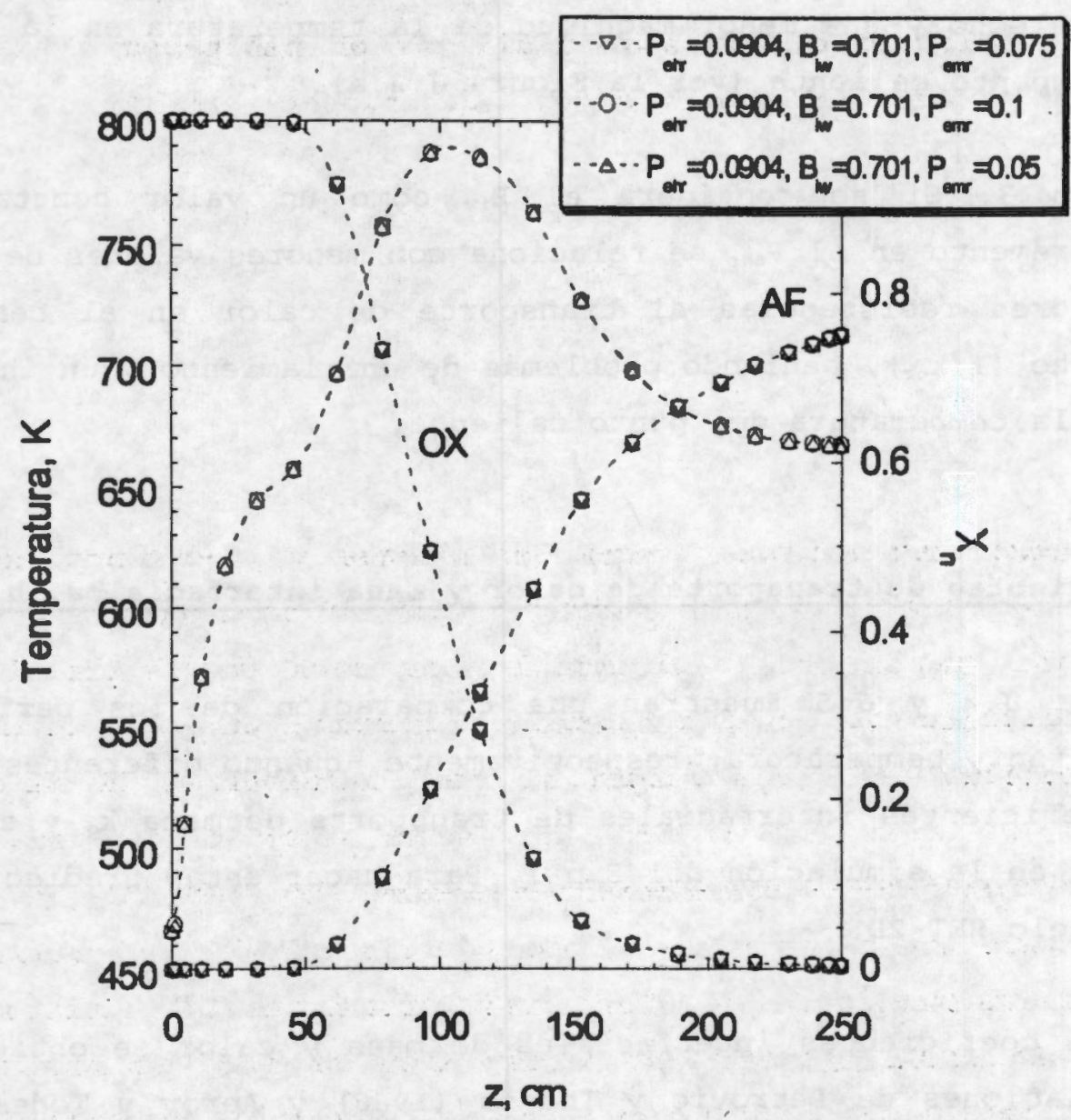

Figura J.3. Efecto del $P_{\operatorname{enx}}$ en los perfiles de temperatura $y$ concentración

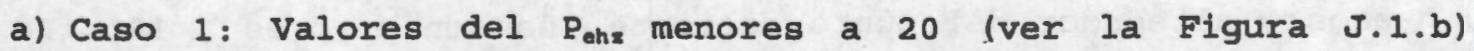
indican la presencia de fenómenos de dispersión en el reactor. Esto se debe a los gradientes de temperatura. Para las condiciones de operación del reactor, el $\mathrm{P}_{\mathrm{ehz}}$ que se estimo es 200, teniendo fenómenos de dispersión despreciables.

b) Caso 2: Si se considera el Pans como un valor constante, un incremento en el $B_{1 w}$ se relaciona con valores de $h_{w}$ mayores a los de $l a k_{\text {err }}$ lo que ocasiona menores resistencias de transporte de calor cerca de la pared $\left(1 / h_{w}\right)$, teniendo un mejor enfriamiento 
del lecho $y$ una menor magnitud de la temperatura en la posición del punto caliente (ver la Figura J.2.a).

c) Caso 3: Si se considera el $B_{14}$ como un valor constante, un incremento en el $P_{\text {ehr }}$ se relaciona con menores valores de la $k_{\text {er }} y$ mayores resistencias al transporte de calor en el centro del lecho $\left(1 / k_{e r}\right)$, teniendo problemas de enfriamiento y un incremento en la temperatura del punto caliente.

\section{$J .2$ Coeficientes de transporte de calor y masa interfaciales, $h_{q}$ y $k_{0}$}

La Figuras J.4 y J.5 muestran una comparación de los perfiles de concentración y temperatura, respectivamente, cuando diferentes valores de los coeficientes interfaciales de transporte de masa $\mathrm{k}_{\mathrm{g}} \mathbf{y}$ calor $\mathrm{h}_{\mathrm{g}}$, se emplean en la simulación del Exp-1. Para hacer estas predicciones se usa el moclelo HET-2D.

Cuando los coeficientes interfaciales de masa y calor se obtienen con las correlaciones de Petrovic y Thodog (1968) Y Aerov y Todes (1968)

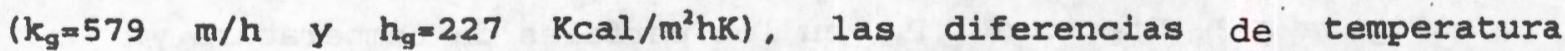
predichas entre la fases sరlida y gaseosa son mayores a $20^{\circ}$. Las observaciones de los perfiles radiales de temperatura en el reactor [14] muestran que las diferencias de temperatura entre las fases solida y gaseosa, alrededor del punto caliente, no son mayores a $10^{\circ}$. Lo que indica un problema con el uso de estas correlaciones. Por lo anterior, se decidio hacer este estudio de sensibilidad de $k_{g} y$ de $h_{g}$, para encontrar aquellos que describan las observaciones; los cuales resultaron ser $k_{g}=1000 \mathrm{~m} / \mathrm{h}$ y $h_{g}=1200 \mathrm{Kcal} / \mathrm{m}^{2} \mathrm{hk}$, mostrando diferencias importantes con aquéllos obtenidos con correlaciones. 

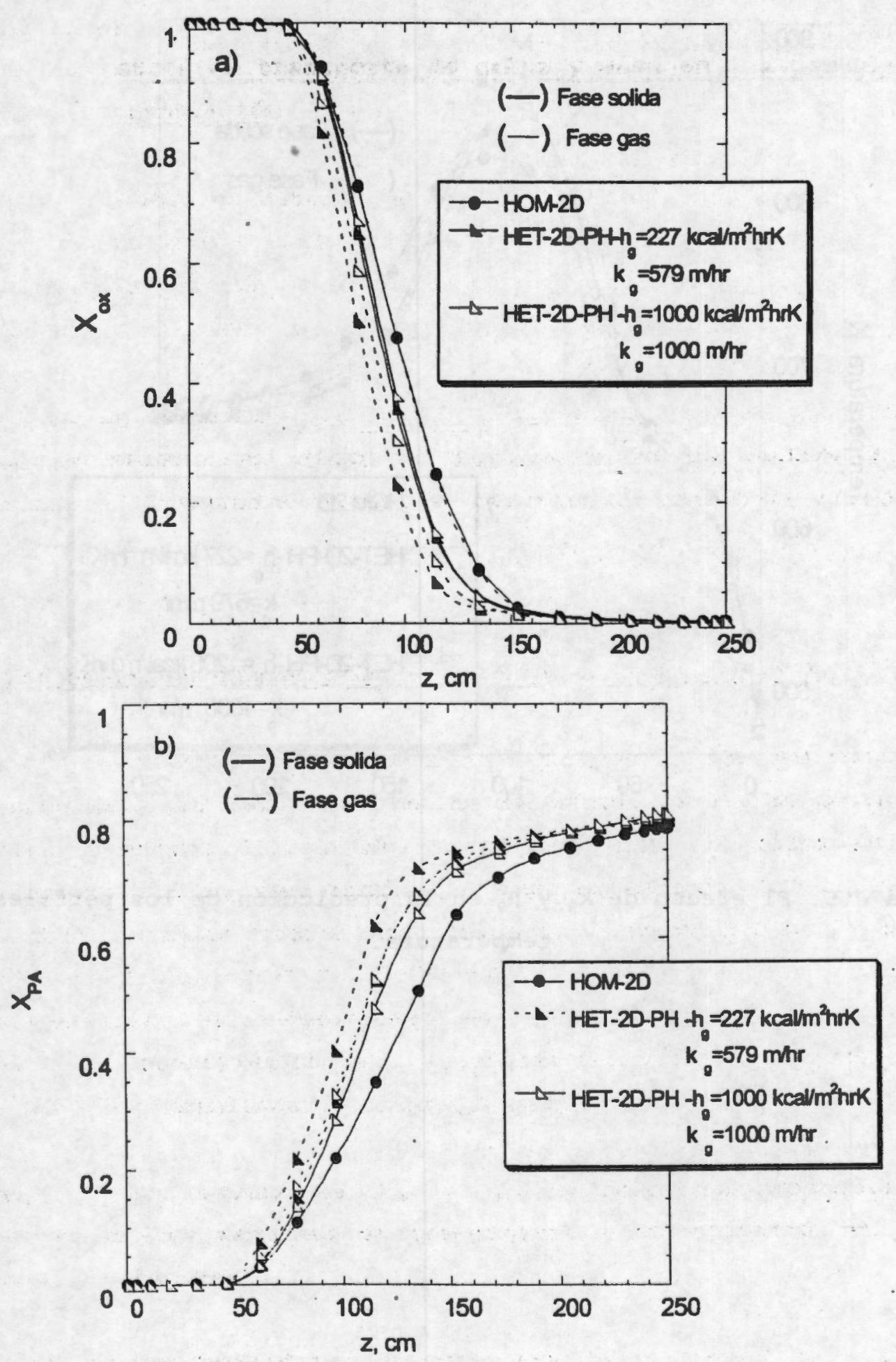

Figura J.4. Efecto de $k_{g} y$ calor $h_{g}$ en la predicción de los perfiles de concentracion: a) de ox $y$ b) de AF. 


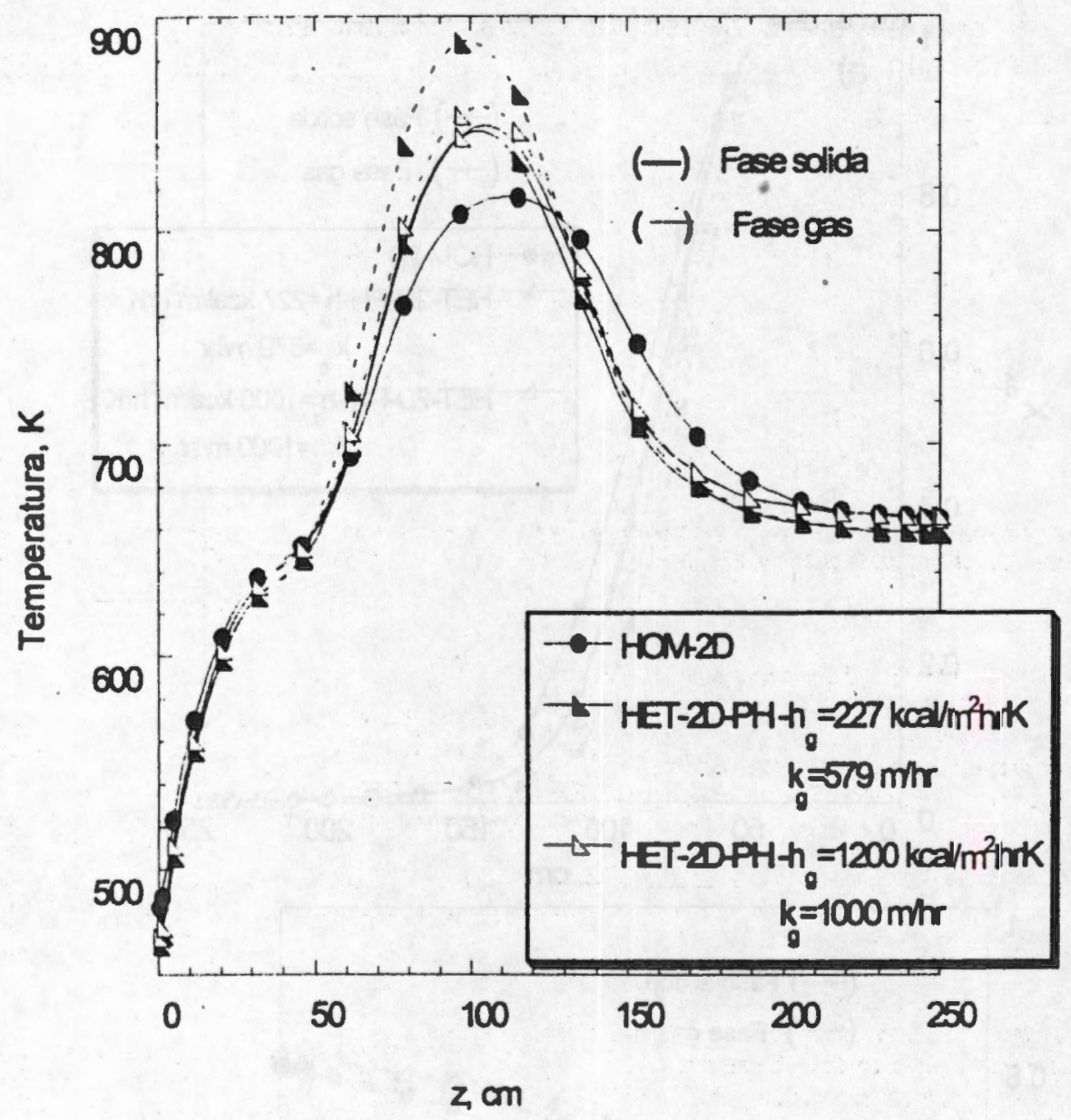

Figura J.5. El efecto de $k_{g} y h_{g}$ en la predicción de los perfiles de temperatura. 


\section{Apéndice $\mathrm{K}$.}

Predicctón de la propuenta SIM-1 a diferentes condlelones de operación

En este Apéndice se hace un estudio de sensibilidad de los perfiles de temperatura y concentración al variar las condiciones de operación del reactor, al utilizar la propuesta SIM-1 y el modelo HET-2D-PH. (ver Capitulo 5) La Figura K.l muestra la predicción del perfil de concentración adimensional y temperatura al utilizar varias concentraciones de alimentación de ox: 0.4\%, 0.7\% y 1r. En la Figura K.1.a se presentan los perfiles de concentración a lo largo del lecho, en donde se observa que los perfiles de concentración muestran el mismo comportamiento, no importando la concentración de entrada de ox, obteniendo los mismos niveles de conversion. Esto indica que siempre se Fienen sitios selectivos a la oxidación parcial o total de ox. En la Figura K.l.b se observa que la magnitud del punto caliente es proporcional a la concentración de alimentación; es decir, entre mayor es la concentración de alimentación de ox, la magnitud del punto caliente incrementa. Este comportamiento es resultado de tener una mayor cantidad de hidrocarburos durante la reacción, teniendo una mayor liberación de calor. Las Figuras K.1.c y K.1.d muestran el comportamiento de los sitios oxidados y reducidos durante la reacción. Se observa que una menor cantidad de sitios selectivos a la oxidación parcial se tiene conforme la concentración de ox incrementa, explicando adecuadamente la desactivación reversible en la superficie del catalizador. En la Figura K.2 se presenta la predicción del perfil de concentración, temperatura $y$ actividad a lo largo del reactor, a dos diferentes temperaturas del baño de enfriamiento. Se observa que el incremento de esta temperatura: no causa cambios apreciables en los perfiles de concentración (ver la Figura K.2.a); que la magnitud del punto caliente se eleve -30 grados y que la superficie del catalizador, en la entrada del lecho, sufre una reactivación (ver la Figura k.2.b); que haya un relativo incremento en la fraccion de sitios selectivos a la oxidación parcial (ver la Figura K.2.c). 

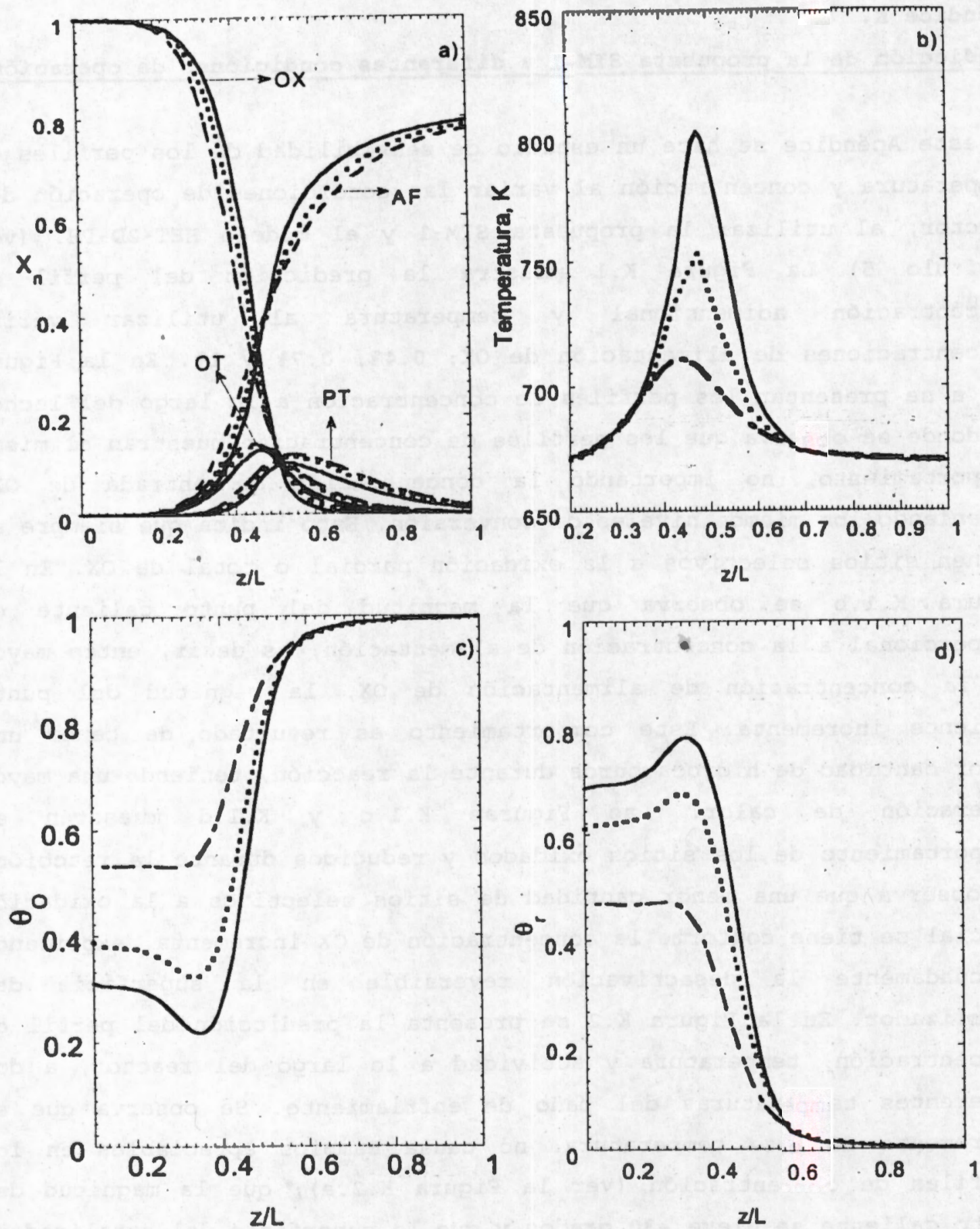

Figura K.1. Comportamiento de los perfiles de temperatura $Y$ concentración adimensional en la fase gas en $\xi=0$ : al variar la concentración de alimentación del ox. $(-) C_{o x}=1$ mol, ( $(--) C_{o x}=0.48 \mathrm{~mol}$. 

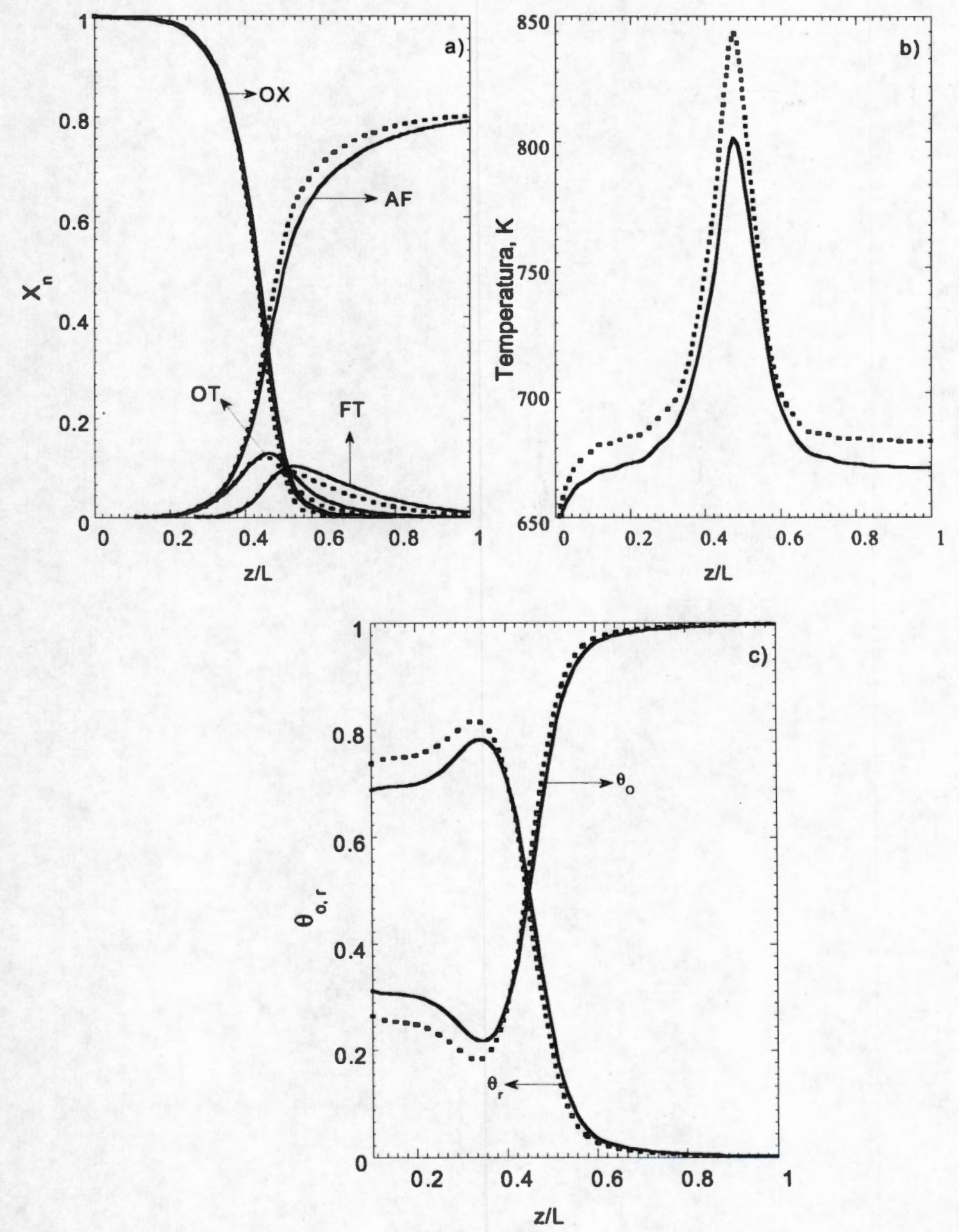

Figura K.2. Comportamiento de los perfiles de temperatura $y$ concentración adimensional en la fase gas en el $\xi=0$, al variar la temperatura del baño de enfriamiento. $(-) T_{w}=669 \mathrm{~K}$ y $(\bullet \bullet) T_{w}=685 \mathrm{~K}$ 


\title{
Heat-Transfer Studies in Packed-Bed Catalytic Reactors of Low Tube/Particle Diameter Ratio
}

\author{
C. O. Castillo-Araiza, ${ }^{\dagger}$ H. Jiménez-Islas, ${ }^{\ddagger}$ and F. López-Isunzat,t \\ Departamento de Ingenieria de Procesos e Hidráulica, Universidad Autónoma \\ Metropolitana-Iztapalapa. México, 09340 D.F., Mexico, and Departamento de Ingenieria \\ Bioquimica, Instituto Tecnológico de Celaya, Guanajuato, Mexico
}

\begin{abstract}
This work describes a heat-transfer study conducted in two beds of low tube/particle diameter ratio $\left(d_{\mathrm{l}} / d_{\mathrm{p}} \approx\right.$ 3) with almost the same diameter but different lengths, packed with catalyst spheres, operating in quasiadiabatic and nonadiabatic modes. The operation in the short bed in a quasi-adiabatic mode provided independent sets of experiments to estimate the effective thermal conductivity at various Reynolds numbers. This information was transferred successfully to model the short and long beds operating in a nonadiabatic mode. The modeling compares the classical approach to model heat transfer in a packed bed with no hydrodynamics, against those which include the hydrodynamics and a radial voidage profile, as well as the boundary layer approximation of this model. Modeling results showed that very similar predictions of radial and axial temperature profiles are obtained in all cases, making it difficult to discriminate between both model approaches.
\end{abstract}

\section{Introduction}

Many experimental studies on heat transfer in packed beds have been performed in the past using pseudo-homogeneous models, searching for correlations to obtain a priori estimates of the effective heat-transfer parameters (wall heat-transfer coefficient $\left(h_{w}\right)$ and thermal conductivity $\left.\left(k_{\mathrm{eft}}\right)\right)$, for the simulation or design of packed-bed catalytic reactors. Some of these experiments have been performed in adiabatic-wall beds, where the axial dispersion of heat is dominant, making use of onedimensional models to estimate the effective axial thermal conductivity. ${ }^{1-4}$ However, most experiments have been performed in nonadiabatic beds, and two-dimensional pseudohomogeneous models have been used to predict the observed axial and radial temperature profiles. ${ }^{3-17}$ In some cases, heterogeneous packed-bed models also have been used. ${ }^{18-22}$

It has been shown, in all these studies, that the parameters $h_{w}$ and $k_{\text {efr }}$ are strongly statistically correlated when they are estimated from the same set of experiments, ${ }^{18.19 .23-28}$ as a recent study has also confirmed. ${ }^{29}$ This type of study constitutes the classical approach to model heat transfer in packed beds, without any hydrodynamic description of the fluid flow in the bed, nor a description of the packed-bed structure. However, in the last 13 years, most of the studies have incorporated the hydrodynamics in the packed bed using Navier-Stokes-type equations that include some description of the porous media, to estimate either $h_{w}$ and $k_{\text {eft }}{ }^{30,31}$ or only $k_{\text {eff }}$ as this type of model makes the existence of $h_{w}$ irrelevant. ${ }^{32-37}$

Several theoretical and experimental studies on the velocity profiles within packed beds have been published since early $1950.9,14,32,38-47$ The use of anemometry techniques allowed the measurement of distortions in the velocity profiles due to voidage profiles in the packed bed, which become larger as the ratio of tube/particle diameter decreases. The zones with larger

- To whom correspondence should be addressed. E-mail: felipe(a) xanum.uam.mx.

${ }^{\dagger}$ Departamento de Ingenieria de Procesos e Hidráulica, Universidad Autónoma Metropolitana-Iztapalapa.

t Departamento de Ingeniería Bioquímica, Instituto Tecnológico de Celaya. velocities corresponds to those with high void fraction, which are normally located near the wall..38,43,48-51 An exact representation of the spatial distribution of the interstitial fluid velocity in packed beds seems to be a very difficult task, and it requires the proper three-dimensional description of the void fraction distribution into the bed at typical values of interest, which, for low rube/particle diameter ratios, are in the range of 0.4-0.5. On the other hand, the use of computational fluid dynamics (CFD) codes can define a computational domain that accommodates the geometries of different types of packing into the bed; however, the numerical solution of the equations to model the flow field in the packed bed requires such a large computer resources that these codes have only been applied to solve short packed beds with large voidage. 35,52,53

It is apparent when modeling highly exothermic reactions in packed beds with low tube/particle diameter ratio, that if radial profiles of velocity, void fraction, and temperature in a packed bed under no reaction conditions are measured, the estimation of the heat-transport parameters should use a model that includes the hydrodynamics and the existing voidage profile; this information then can be transferred to model the catalytic reactor.

This work involves the estimation of heat-transfer parameters in a packed-bed with low tube/particle diameter ratio $\left(d_{V} / d_{p} \approx\right.$ 3), packed with spheres of an industrial $\mathrm{V}_{2} \mathrm{O}_{5} / \mathrm{TiO}_{2}$ catalyst (from von Heyden), used in the partial oxidation of o-xylene to phthalic anhydride. First, the classical approach is used to predict radial and axial temperature profiles. Second, these results are compared to those obtained when the hydrodynamics and an empirical expression for the radial void fraction profiles are coupled to heat transfer in the packed-bed model. The study is based on two independent sets of experiments performed in the same packed bed, for particle Reynolds numbers $\left(R e_{p}\right)$ in the range of $25-630$ and temperatures in the range of $300-673 \mathrm{~K}$. The first set of experiments was performed in a short adiabaticwall packed bed, following the studies of Yagi, Kunii, and Wakao, ${ }^{1,2}$ Vortuba et $\mathrm{al},{ }^{3}$ and Vortmeyer and Adam, where the heating was applied at the top of the bed by means of an infrared lamp, and temperature measurements were taken at several axial positions at the axial axis of the bed. assuming 


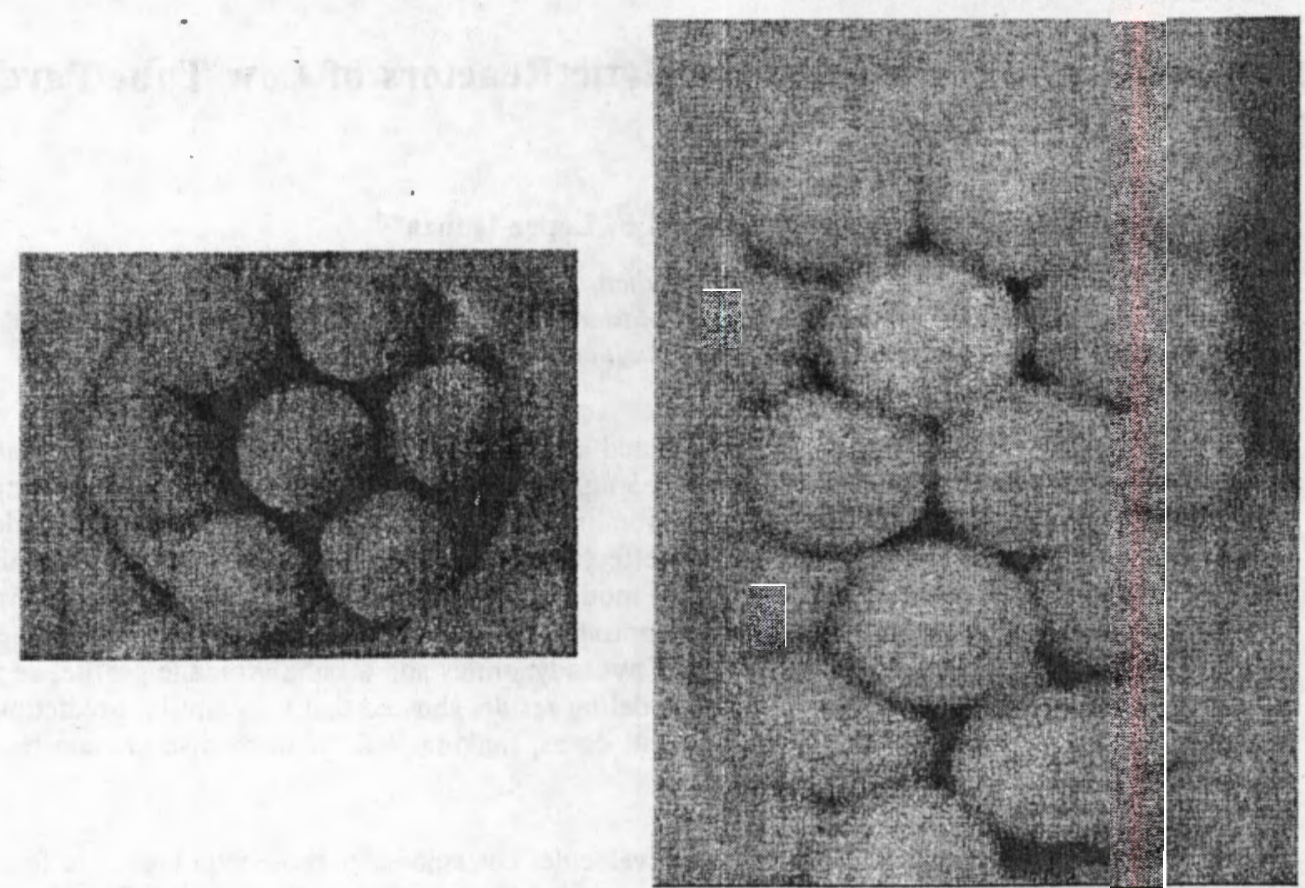

Figure 1. Photographs showing the top and side views of an acrylic-tube packed bed of same dimensions as the experimental system used in the heattransfer experiments.

the walls are adiabatic. The second type of experiments were performed by heating the same bed through the tube wall, and axial and radial temperature measuremènts were taken during the transients until a steady state axial temperature profile was reached. $6,18,19,22,29,54-56$ Some of the experiments reported in this work are taken from previous works that involved heat-transfer studies in an industrial packed-bed catalytic reactor, using the same $\mathrm{V}_{2} \mathrm{O}_{5} / \mathrm{TiO}_{2}$ catalyst spheres, ${ }^{21,22}$ with similar tube diameter but a longer packed length, which is heated by a molten salt bath; these studies also provided relevant data on the pressure drop in this packed bed at various air flow rates.

Following the classical approach, the first set of experiments allowed the estimation of the axial thermal conductivity, because this is the only parameter in the adiabatic packed-bed model. For the second set of nonadiabatic experiments, two cases were considered: (i) $h_{w}$ was estimated as the only parameter using the value of $k_{\text {eff }}$ obtained from the modified Yagi, Kunii, and Wakao' (YKW) correlation, based on the quasi-adiabatic experiments; (ii) the values of $k_{\text {erf }}$ obtained from the quasiadiabatic experiments were used as initial estimates in the simultaneous estimation of $k_{\text {eff }}$ and $h_{w}$ for the nonadiabatic experiments. In the first case, only one parameter is estimated for each set of experiments, which avoids having the high correlation between both parameters. In the second case, both parameters are estimated simultaneously, using the infornation obtained from the quasi-adiabatic experiments. In the second part, the prediction of axial and radial temperature profiles oblained with the classical approach (no hydrodynamics) are compared to those obtained by including a momentum equation that contained the Darcy, Forchheimer, and Brinknan terms for the axial and radial velocity components, and to its boundary layer approximation, which only considers the axial velocity component. The modified YKW' correlation was used to estimate the axial Peclet number for the several mass flow rates in the quasi-adiabatic experiments, to predict the temperature measurements in the large and short nonadiabatic beds. Here, it is assumed that. given the large voidage distribution around the packing in the bed, and particularly for high Reynolds numbers, the bed behaves similar to an isotropic medium; therefore, a single value of $k_{\text {eff }}$ suffices to calculate the heat transport in the entire packed bed.

\section{The Experimental Systems}

The main experimental system used in this study is a tube $41 \mathrm{~cm}$ in length, having an internal diameter of $2.56 \mathrm{~cm}$, packed with $8.2 \mathrm{~mm}$ spheres of a nonporous industrial catalyst $\left(\mathrm{V}_{2} \mathrm{O}_{5}\right)$ $\mathrm{TiO}_{2}$ ), yielding a ratio of $d_{\mathrm{V}} / d_{\mathrm{p}}=3.122$ and a measured average bed porosity of 0.44 . Figure 1 show top and side views of a packed acrylic tube with the same dimensions as the stainless steel short packed bed; here, large radial and axial void fraction profiles can be observed near the tube wall and around the central annular core at the tube axis. It is expected that the large voidage around the packing produce important changes in the radial, angular and axial velocity components in the packed bed. In this experimental system the two types of experiments were performed, similar to those conducted in other studies. 13,6,18,38

In the first set of experiments, the packed bed was operated in a quasi-adiabatic mode. The bed, which is enclosed by the thermal insulation jacket of the electrically heated fumace (with the elements switched off), was heated at the top using an infrared lamp, and all the experiments were performed using air flowing from the bottom of the tube. The inlet air temperature was kept constant to $302 \pm 1 \mathrm{~K}$. The axial temperature profile at the central axis of the tube and wall temperatures were measured with 10 Type $K$ thermocouples, placed at several axial locations along the bed, which can be move along the tube radius (Figure 2); the measurements were taken every $15 \mathrm{~s}$ and the steady state was attained within $\sim 1 \mathrm{~h}$. A series of experiments, performed at different flow rates of air, are given in Table 1.

The second set of experiments was performed in the short packed bed, but this time, operating in a nonadiabatic mode, by heating the bed through the wall by the electric furnace, at a controlled set point temperature of $673 \pm 3 \mathrm{~K}$ located at half the length of the bed; this temperature was chosen to be close to that of the salt bath temperature used in the experiments with 


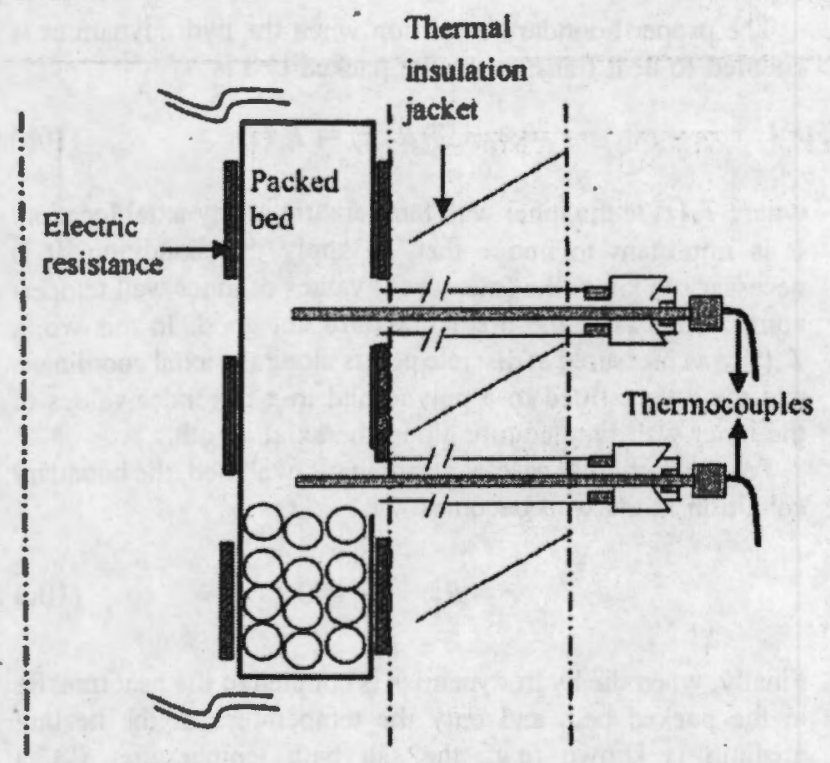

Figure 2. Schematic diagram of the experimental packed-bed system showing the positioning of the thermocouples during the operation in the adiabatic-wall bed and the nonadiabatic bed.

Table 1. Experimental Conditions for the Adiabatic-Wall and Nonadiabatic Packed Beds

\begin{tabular}{|c|c|c|c|}
\hline \multicolumn{2}{|c|}{ Adiabatic-Wall Packed Bed } & \multicolumn{2}{|c|}{ Nonadiabatic Packed Bed } \\
\hline experiment & $R e_{p}^{e}$ & experimeat & $R_{e}^{p}$ \\
\hline 1 & 0 & 7 & 25 \\
\hline 2 & 80 & 8 & 60 \\
\hline 3 & 190 & 9 & 160 \\
\hline 4 & 360 & 10 & $630^{\circ}$ \\
\hline 5 & 510 & & \\
\hline 6 & 630 & & \\
\hline
\end{tabular}

“Particle Reynolds number. Experiments in an industrial fixed-bed cakalytic reactor, taken from ref 22 .

the nonadiabatic large bed. ${ }^{14,22}$ In both beds, the heat was transferred from the wall into the packed bed, producing temperature gradients of different magnitude in axial and radial directions. During the performance of the entire set of nonadiabatic experiments in the short bed, the heating through the tube wall along the axial length was not uniform; under conditions of no air flow, the heating of the bed showed a maximum in the axial temperature at half the tube length, where the set-point measurement was located. During the experiments with flowing air, this maximum was displaced toward the bed's exit, and its location was dependent on the magnitude of the rate of air flow, which resembles that of a packed-bed catalytic reactor with a hot spot at some point along its axial length. To study the heat transport in a nonadiabatic mode under different mass flow rate conditions, this was yaried within a smaller range, as compared to those experiments in the quasi-adiabatic mode of operation. Temperature measureinents were taken at several axial and radial positions into the bed for all flow rates. Additional information of experiments performed in the large bed, concerning the pressure drop under no reaction conditions at several flow rates of air, at a salt bath temperature of $663 \mathrm{~K}$, was taken from a previous work; ${ }^{22}$ these experiments were performed in a packed bed $260 \mathrm{~cm}$ in length and $2.54 \mathrm{~cm}$ in diameter, with a tube/particle diameter ratio, $d / d_{p}=3.097$, and a measured average bed porosity of 0.5 , which, for the sake of completeness, are given in Figure 3. Other results of axial and radial temperature profiles measured under no reaction conditions $^{\text {it }}$ were also used in this study.

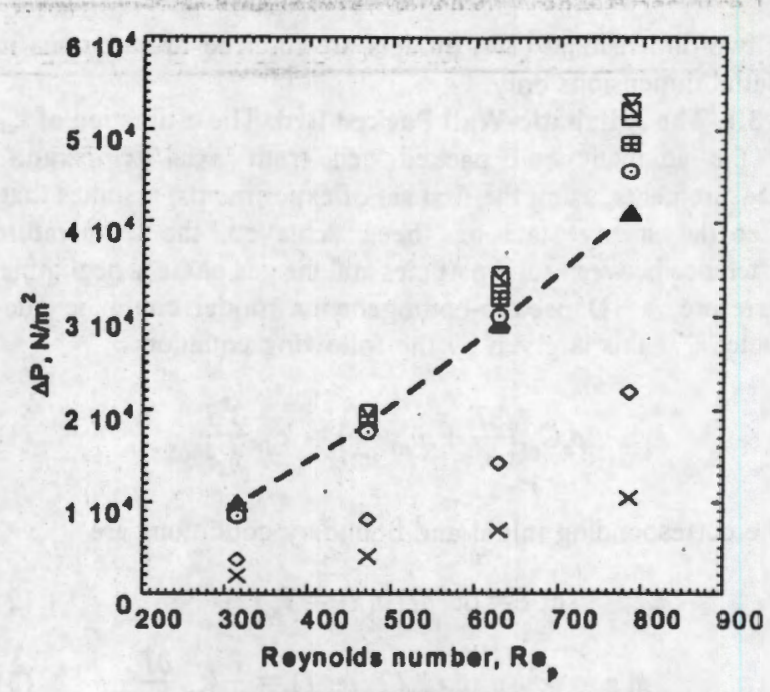

\begin{tabular}{l}
$\Rightarrow$ - Experimental measuraments \\
$\Delta$ This work \\
$\square$ Ergun (1952) \\
0 Delmas and Froment (1988) \\
$\Delta$ Leva (1959) \\
$\nabla$ Macdonald ot al. (1979) \\
$\times$ Eisfeld et al. (2005) \\
Fround et al. (2003) \\
$\circ$ Elsfold et al. (2001) \\
\hline
\end{tabular}

Flgure 3. Experimental resuits compared with theoretical curves for difierent correlations for the pressure drop in the industrial packed bed at different particle Reynolds number $\left(R e_{p}\right)$ values.

\section{Modeling the Heat Transport in a Packed Bed}

This work has two objectives. One is to determine the contribution of the hydrodynamics and radial voidage distribution in the packed-bed heat-transfer models, with reference to the classical approach, by comparing predicted versus measured temperature profiles in both beds; the other objective is to show the role of the quasi-adiabatic experiments by providing independent estimates of the effective thermal conductivity for the modeling of the nonadiabatic beds with the same tubediameter-to-particle-diameter $\left(d_{V} / d_{p}\right)$ ratio, which could be used in the modeling of a packed-bed catalytic reactor for partial oxidation reactions of similar dimensions.

First, the classical approach is applied, assuming that the flow velocity and void fraction in the entire bed are both constant. Second, the hydrodynamics in the bed is coupled to heat transport in the model for the packed bed, together with a radial voidage profile (given by an empirical expression from the literature). In all cases, it is assumed that the fluid has constant thermodynamic properties: the viscosity and density are constant, and the fluid is incoinpressible and Newtonian. As mentioned previously, it is assumed that the $k_{\text {er }}$ values obtained. from the adiabatic-wall bed experiments can be used to describe radial and axial temperature profiles in the nonadiabatic beds with similar $d_{\delta} / d_{p}$ ratio; although this assumption may be questionable, good predictions are obtained as shown below. In all cases, the simultaneous estimation of $k_{e f r}$ and $h_{w}$ was performed using always as initial estimates for $k_{\text {eff }}$ the values obtained from the quasi-adiabatic experiments. For some of the modeling cases in which the hydrodynamics is described by the radial and axial velocity components, it is assumed that measurements of the wall teinperature in the bed are not available; therefore, a boundary layer approximation is introduced, which makes use of the heat-transfer coefficient at the wall $\left(h_{w}\right)$. In the following, the so-called one-dimensional (ID) 
or two-dimensional (2D) models are referred to variations in spatial dimensions only.

3.1. The Adiabatic-Wall Packed Bed. The estimation of $k_{\text {efr }}$ in the adiabatic-wall packed bed from axial temperature measurements, using the first set of experiments, assumes that, after the steady state has been achieved, the temperature difference between solid particles and the gas phase is negligible, therefore, a ID pseudo-homogeneous model could be adequate; $^{-18-20}$ this is given by the following equation:

$$
\rho_{\mathrm{f}} C_{\mathrm{pr}}\left(\frac{\partial T}{\partial t}+v_{\mathrm{z} 0} \frac{\partial T}{\partial z}\right)=k_{\mathrm{cff}} \frac{\partial^{2} T}{\partial z^{2}}
$$

The corresponding initial and boundary conditions are

$$
\begin{gathered}
\text { at } t=0: \quad T(0, z)=T_{\mathrm{ss}}(z) \\
\text { at } z=0: \quad v_{20 \rho \mathrm{p}} C_{\mathrm{pl}}\left(T_{0}-T\right)=-k_{\mathrm{err}} \frac{\partial T}{\partial z} \\
\text { at } z=L: \quad k_{\mathrm{eft}} \frac{\partial T}{\partial z}=q_{0}
\end{gathered}
$$

where $v_{z 0}$ is the value of the axial velocity component at $r=0$ obtained from the solution of the equation of motion for the axial component given by eqs $12-22 ; q_{0}$ is the heat flux supplied by the infrared lamp at the top of the bed. Model predictions of the axial temperature profiles and the estimation of the effective thermal conductivity for this model are discussed below.

3.2. The Nonadiabatic Packed Bed. The prediction of the measured radial and axial temperature profiles in the nonadiabatic packed bed, using the second set of experiments, also assumes that the temperature difference between solid and gas phases is negligible. For its description, a 2D pseudohomogeneous model is used, which accounts for the axial and radial dispersion and the convective transport of heat. This model can also be used in the description of the adiabatic-wall packed bed with the proper boundary condition at the wall. The nonadiabatic model is given by the next expression:

$$
p_{\mathrm{p}} C_{\mathrm{pr}}\left(\frac{\partial T}{\partial t}+v_{z} \frac{\partial T}{\partial z}+v_{\mathrm{r}} \frac{\partial T}{\partial r}\right)=k_{\mathrm{eff}}\left(\frac{\partial^{2} T}{\partial r^{2}}+\frac{1 \partial T}{r \partial \mathrm{r}}+\frac{\partial^{2} T}{\partial z^{2}}\right)
$$

The corresponding initial and boundary conditions are

$$
\begin{aligned}
& \text { at } t=0: T(0, r, z)=T_{s s}(r, z) \\
& z=0: \quad v_{z} \rho C_{p} r\left(T_{0}-T\right)=-k_{\text {efr }} \frac{\partial T}{\partial z} \\
& z=L: \quad \frac{\partial T}{\partial z}=0 \\
& r=0: \quad \frac{\partial T}{\partial r}=0
\end{aligned}
$$

where $v_{z}$ and $v_{r}$ are the velocity components in the axial and radial directions, respectively. For the case of the classical

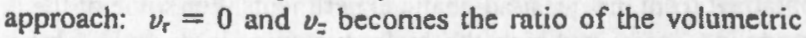
flow rate divided by the cross-sectional area of the bed. For this model, three possible boundary conditions at the wall can be used, For the classical approach, this condition is as follows:

$$
r=R_{\mathrm{T}}: \quad-k_{\mathrm{eff}} \frac{\partial T}{\partial r}=h_{\mathrm{w}}\left(T-T_{\mathrm{w}}\right)
$$

The proper boundary condition when the hydrodynamics is coupled to heat transport in the packed bed is

$$
r=R_{\mathrm{T}}: \quad T\left(R_{\mathrm{T}}, z\right)=T_{\mathrm{w}}(\mathrm{z})
$$

where $T_{w}(z)$ is the inner wall temperature at any axial location. It is important to notice that, to apply this condition, it is necessary to know the (measured) values of inner wall temperatures; otherwise, the predictions are not good. In this work, $T_{\mathrm{w}}(z)$ was measured at discrete points along the axial coordinate, and they were fitted to a polynomial in $z$ to render values of the inner wall temperature along the axial length.

For the particular case of an adiabatic-wall bed, the boundary condition at the wall becomes

$$
r=R_{\mathrm{T}}: \quad \frac{\partial T}{\partial r}=0
$$

Finally, when the hydrodynamics is coupled to the heat transfer in the packed bed, and only the temperature of the heating medium is known (e.g.s. the salt bath temperature, $T_{\mathrm{b}}$ ), a boundary layer approxination to the momentum equations could be applied, using the boundary conditions described by eq 10a with a constant value for $T_{w}=T_{b}$.

3.3. The Hydrodynamies and the Voidage Profile in the Packed Bed. The equations of motion for the radial and axial velocity components in the packed bed are given by a NavierStokes type of equation. which include the Darcy and Forchheimer terms ${ }^{58,39}$ (in terms of Ergun), and the viscous dissipation is given by Brinkman's expression ${ }^{60}$ without using an effective viscosity coefficient, as it is explained below.

Mass continuity:

$$
\frac{1}{r} \frac{\partial}{\partial r}\left(r v_{\mathrm{r}}\right)+\frac{\partial}{\partial z}\left(v_{z}\right)=0
$$

Momentum equation for component z:

$$
\begin{aligned}
\rho_{\mathrm{f}} \epsilon\left[\frac{\partial v_{\mathrm{z}}}{\partial t}+v_{\mathrm{f}} \frac{\partial v_{z}}{\partial r}+v_{z} \frac{\partial v_{z}}{\partial z}\right] & =-\epsilon \frac{\partial \mathrm{p}_{\mathrm{z}}}{\partial z}+ \\
\epsilon \mu_{\mathrm{f}} \nabla^{2} v_{z} & -\epsilon \frac{\mu_{\mathrm{f}}}{K} v_{z}-\epsilon^{2} \frac{\rho_{\mathrm{f}}}{K_{\mathrm{z}}} \nu_{z}^{2}+\epsilon \rho_{f} g_{z}
\end{aligned}
$$

Momentum equation for component $r$ :

$$
\begin{aligned}
\rho_{\mathrm{r}} \epsilon\left[\frac{\partial \nu_{\mathrm{r}}}{\partial t}+\nu_{\mathrm{r}} \frac{\partial \nu_{r}}{\partial r}+\nu_{z} \frac{\partial \nu_{\mathrm{r}}}{\partial z}\right] & =-\epsilon \frac{\partial p_{\mathrm{r}}}{\partial r^{*}}+ \\
& \epsilon \mu_{\mathrm{r}} \nabla^{2} \nu_{\mathrm{r}}-\epsilon \frac{\mu_{\mathrm{f}}}{K} \nu_{\mathrm{r}}-\epsilon^{2} \frac{\rho}{K_{z}} \nu_{r}^{2}
\end{aligned}
$$

The corresponding initial and boundary conditions are

$$
\begin{aligned}
\text { at } t=0: & v_{z}(0, r, z)=v_{z a s}(r, z) ; \quad v_{r}(0, r, z)=v_{r w s}(r, z) \\
r=0: & \frac{\partial u_{z}}{\partial r}=\frac{\partial v_{r}}{\partial r}=0 \\
r=R_{t}: & v_{z}=v_{r}=0 \\
z=0: & v_{z}=\left\langle u_{z}(r)\right\rangle ; v_{r}=0 \\
z=L: & \frac{\partial v_{z}}{\partial z}=\frac{\partial v_{r}}{\partial z}=0
\end{aligned}
$$

The permeability parameters for the packed bed $\left(K\right.$ and $K_{2}$, in eqs 12 and 13) are given, in terms of Ergun relations, ${ }^{61}$ by 


$$
\begin{aligned}
& K=\frac{\epsilon^{3} d_{p}^{2}}{\alpha(1-\epsilon)^{2}} \\
& K_{z}=\frac{\epsilon^{3} d_{p}}{\beta(1-\epsilon)}
\end{aligned}
$$

In this work, the constants $\alpha$ and $\beta$ were determined by fitting the Ergun model 61 to the experimental data of the pressure drop in the large bed at several air mass flow rates. In this way, the need for using an effective viscosity term in the Brinkman's expression was avoided. Moreover, it was assumed that the radial pressure gradient in eq 13 is negligible.

To describe the radial voidage profile in the packed bed, the empirical expression developed by de Klerks1 was chosen, although other correlations were also tested. The radial void fraction profile $\epsilon(r)$ is given by

$$
\begin{aligned}
& \text { for } \frac{R_{\mathrm{T}}-r}{d_{\mathrm{p}}}<0.637: \\
& \qquad \epsilon(r)=2.14\left(\frac{R_{\mathrm{T}}-r}{d_{\mathrm{p}}}\right)^{2}-2.53\left(\frac{R_{\mathrm{T}}-r}{d_{\mathrm{p}}}\right)+1(21) \\
& \text { for } \frac{R_{\mathrm{T}}-r}{d_{\mathrm{p}}}>0.637: \epsilon(r)=\epsilon_{\mathrm{os}}+ \\
& 0.29 \exp \left[-0.6\left(\frac{R_{\mathrm{T}}-r}{d_{\mathrm{p}}}\right)\right]\left(\cos \left(2.3 \pi\left[\frac{R_{\mathrm{T}}-r}{d_{\mathrm{p}}}-0.16\right]\right)\right)+ \\
& \quad 0.15 \exp \left[-0.9\left(\frac{R_{\mathrm{T}}-r}{d_{\mathrm{p}}}\right)\right]
\end{aligned}
$$

Equations 11-18 describe the hydrodynamics in the packed bed, which, together with expressions 21 and 22, and coupled to eqs $5-10$, described the axial and radial temperature profiles in a nonadiabatic packed bed.

All the models used in this work are described by parabolic partial differential equations (PDEs), which were solved numerically by the method of orthogonal collocation, using $\mathbf{3 0}$ and $\mathbf{5 0}$ interior collocation points in the radial and axial coordinates, respectively, employing shifted Legendre polynomials. ${ }^{62,63}$ The reduced set of ordinary differential equations (ODEs) in each case was solved by the Runge-Kutta-Fehlberg method. ${ }^{64}$ The estimation of the heat-transport parameters was performed by the Levenberg-Marquardt method. ${ }^{65}$

\section{Results and Discussion}

The following discussion involves the criteria for the estimation of the effective heat-transport parameters. The first set of experiments in the adiabatic-wall bed provided independent estimates of $k_{\text {efr, }}$ as a function of the particle Reynolds number $\left(R e_{p}\right)$, which could be used directly in the modeling of the nonadiabatic bed; the previous discussion applies in the same way to models regardless of whether they do or do not include the hydrodynamics and radial voidage profiles. The predictions of the observed axial and radial temperature profiles were performed for packed beds with different lengths ( 41 and 260 $\mathrm{cm})$ and a similar tube/particle diameter ratio $\left(d \sqrt{ } / d_{p}=3.122\right.$ and 3.097) packed with the same catalyst spheres $\left(d_{p}=8.2\right.$ $\mathrm{mm})$. Section 4.1 analyzes the prediction of measurements in the adiabatic-wall packed bed and gives an estimation of $k_{\mathrm{err}}$. in terms of the modified YKW correlation, ${ }^{1}$ given by eq 23.

\begin{tabular}{|c|c|c|c|c|c|c|}
\hline$R e_{p}^{\prime \prime}$ & Cases $A$ & Case IA & Case IB & Case 2B & Case $3 A$ & Case 3B \\
\hline \multicolumn{7}{|c|}{ Peclet Number, $\mathrm{Pe}$} \\
\hline 25 & 4.27 & & 4.28 & 4.96 & & 3.97 \\
\hline 60 & 5.12 & & 7.1 & 7.89 & & 6.95 \\
\hline 160 & 7.52 & & 7.5 & 9.12 & & 10.01 \\
\hline 630 & 15.5 & & 16.5 & 14.12 & & 14.15 \\
\hline \multicolumn{7}{|c|}{ Wall Biot Number, $B i_{w}$} \\
\hline 25 & & 9.11 & 9.12 & & 9.81 & 7.38 \\
\hline 60 & & 7.81 & 8.51 & & 7.52 & 6.51 \\
\hline 160 & & 5.43 & 4.32 & & 4.41 & 3.09 \\
\hline 630 & & 0.82 & 0.72 & & 1.45 & 1.35 \\
\hline
\end{tabular}
Section 4.2 considers the nonadiabatic bed, assuming plug-flow and constant void fraction, and analyzes two cases where $h_{w}$ is

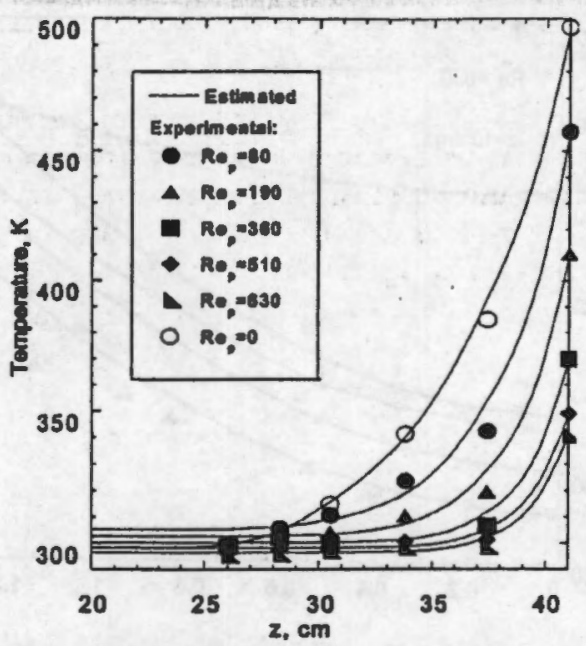

Figure 4. Experimental results compared with predicted axial temperature profiles in the adiabatic-wall packed bed at different $R e_{\mathrm{p}}$ values.

Table 2. Estimated Values of the Peclet and Wall Biot Numbers

- Particle Reynolds number.

always estimated, whereas $k_{e f r}$ is either estimated simultaneously with $h_{w}$ or it is calculated with eq 23. Section 4.3 involves the hydrodynamics and radial voidage distribution in the packedbed models and compares the predictions of the heat transport in the short packed bed with and without the incorporation of the hydrodynamics in the model, at small Reynolds numbers $\left(R e_{\mathrm{p}} \leq 160\right)$. Section 5 shows the prediction of temperature profiles in the large bed, based on previous estimates from the short bed, comparing the results from the classical approach to those when the hydrodynamics are also used, at a high Reynolds number $\left(R e_{\mathrm{p}}=630\right)$. The estimated values of the Peclet $(P e)$ and wall Biot $\left(B i_{\mathrm{w}}\right)$ numbers for all cases are given in Table 2.

4.1. Heat Transport in the Adiabatic-Wall Packed Bed. The comparison between the solutions with the model given by eqs $1-4$, with those obtained from eqs $5-10$ c, coupled to eqs $11-22$, showed no significant differences in the prediction of the axial temperature measurements $(1-3 \mathrm{~K})$; therefore, the former model was used in the prediction of the measured axial temperature profiles in the adiabatic-wall bed, following the YKW' approach. The results are shown in Figure 4 for conditions given in Table $\mathrm{I}$. The estimated values of the effective thermal conductivity at different air tlow rates can be expressed in terms of the particle Reynolds numbers $R e_{p}$, by modifying the magnitude of the empirical parameter $\delta$ in the YKW correlation given below:

$$
\frac{1}{P e}=\frac{1}{P e^{0}}+\frac{\delta}{P e_{\mathrm{f}}}\left(R e_{\mathrm{p}} P r\right)
$$

where $P_{e}{ }^{\circ}$ and $P_{e f}$ are the Peclet numbers evaluated using the thermal conductivity of the bed under no-flow conditions and the fluid (air) conductivity, respectively. The estimated value 

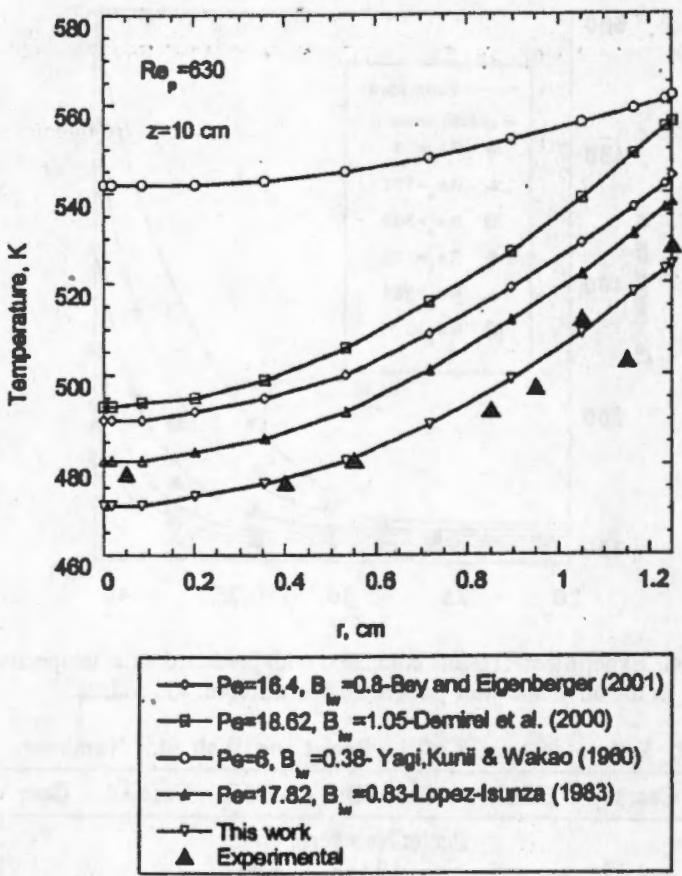

Figure 5. Experimental results compared with predicted radial temperature profiles using different correlations to calculate the Peclet number $(P e)$ and wall Biot number $\left(B i_{w}\right)$ values.

of the empirical parameter $\delta$ was 0.075 in this work; its original value is 0.8 for $d v d_{p} \approx 10$. Other reported values vary from $50 \%$ to $300 \%$ for beds where $d_{v} / d_{p}<10$ and $d_{1} / d_{p}>10$, respectively. $1,17,27,29,66,67$ It is interesting to note that the hydrodynamic effects for this particular packed bed with $d / d d_{p}$ $\approx 3$ can be lumped into the single parameter $\delta$, which is expected to account for the entire effect of axial velocity gradients on the rate of the heat transport close to the wall, where the larger voidage is located. The comparison between the predicted values for the Peclet number at different $R_{e}$ values, using several correlations published in the literature, showed large differences with the estimated values obtained in this work. The YKW correlation with $\delta=0.075$ in eq 23 was used in the prediction of the temperature profiles in all the cases for the nonadiabatic bed, to provide values for $k_{e f}$ that were used either directly in the simulations or as initial estinuates.

4.2. Heat Transport in the Nonadiabatic Packed Bed. Calculations of temperature profiles in the nonadiabatic packed bed, assuming that flow velocity and void fraction are constants, were performed using eqs $5-10 \mathrm{a}$, which, together with the second set of experiments, and eq 23, allowed the estimation of $k_{\mathrm{cff}}$ and $h_{\mathrm{w}}$. The prediction of observed axial and radial temperature profiles (not shown) at $R e_{p}=25-160$ showed that there is practically no difference when the value for $k_{\text {err }}$ is taken directly from eq 23 and $h_{w}$ is the only estimated parameter, versus when both $k_{e r r}$ and $h_{w}$ are estimated simultaneously, although the former yielded better estimation of the radial profiles (and it is slightly better for the axial profile too). It was deternined that the predictions of the axial temperature profiles are reasonably good; however, it was found that predictions become less satisfactory as $R e_{\mathrm{p}}$ increases, particularly for those measurements near the bed's exit, which was affected by some heat losses due to cooling with the extremes of tube connections with the packed bed.

Figure 5 compares the prediction of the radial temperature profile in the large bed for $R e_{\mathrm{p}}=630$, using different correlations from the literature to calculate $k_{c \pi}$ and $h_{w}, 1,17,27,29,66,67$ with that obtained following this approach, and with a previous

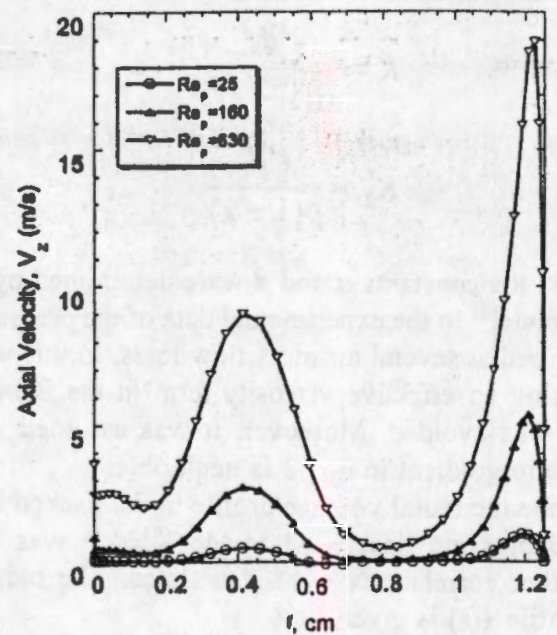

Figure 6. Computed radial profiles for the axial velocity component at different $R e_{\mathrm{p}}$ values.

prediction obtained by estimating both parameters using a pseudo-heterogeneous model to predict pure heat-transfer experiments. ${ }^{22}$ The predictions obtained with other correlations show large differences between the experimental and predicted profiles, and it seems that this failure is due to the fact that these correlations were not obtained from experiments with low $d v d_{\mathrm{p}}$ ratios, as in this work.

4.3. Radial Void Fraction Profiles and the Hydrodynamies in the Packed Bed. Packed beds with low $d f d_{p}$ ratios have regions with large voidage, as shown in Figure 1; one region is near the wall with a size of approximately half a particle diameter, whereas the other is located at a distance of one particle diameter from the wall and surrounds the sphere at the center of the tube diameter. In these regions, interstitial fluid velocity, convective heat transport, and viscous effects are expected to be larger. Because of this observation, the shape of the radial temperature profiles could be different from those computed using constant values for the interstitial velocity and void fraction, and it may also give different estimates of the heat-transport parameters.

To determine the role of radial variations in the packed bed porosity in the prediction of temperature profiles, these are included in the model using the empirical expression given by de Klerk, s! which was chosen among others, because it describes measurements such as those made by Benenati and Brosilow, ${ }^{49}$ and it is expected to give a reasonable description of the radial variations in voidage for the system shown in Figure 1. In the computation of the velocity fields in the packed bed using the de Klerk expression, ${ }^{51}$ it is assumed that only the axial and radial velocity components in the momentum equation are significant. In these calculations, the pressure-drop data from the large packed bed ${ }^{22}$ were used to estimate the values of the Ergun constants for this system, which yielded the values $\alpha=1028$ and $\beta=1.15$; these values assign a larger contribution to the viscous dissipation, rather than to the convective term. Figure 6 gives the radial profiles at steady state for the axial velocity component, showing two large peaks in those regions with larger voidage; it also gives the corresponding average velocity at several $R e_{p}$ values. On the other hand, the computed value of radial velocity component was very small, and it was found that it approaches to zero in most of the axial length of the bed, just after the first particle layer, perhaps because of the lack of a source tern in this equation, because the radial pressure gradient was considered to be negligible; however, similar results have been obtained by other researchers. ${ }^{30,42,69}$ 
The main aspects to be-addressed in the design of the class of packed-bed catalytic reactors with a low $d v d d_{p}$ ratio for highly exothermic reactions are (i) the kinetics of the catalytic and deactivation reactions, and (ii) the transport of momentum, mass, and energy. In this respect, and ignoring the reaction kinetics at this time, it has been argued that, for the correct prediction of the measured axial and radial temperature profiles in reactors for partial oxidation reactions, the reactor model should include not only axial and radial dispersion of heat but also the hydrodynamics and void fraction profiles in the packed bed. ${ }^{1330,69.70}$ With these in mind, and to show the role of adiabatic-wall bed experiments in the prediction of effective thermal conductivities in nonadiabatic beds, which could be successfully used in the simulation and design of these as catalytic reactors, the following six cases are analyzed, in which the hydrodynamies plus the radial void fraction profile are included in the description of the heat transfer in a nonadiabatic packed bed model; these cases are compared to experiments and to predictions from the classical approach, when $R e_{\mathrm{p}}=160$ :

Case IA: The classical approach. Here, $h_{w}$ is the only parameter estimated, and $\boldsymbol{k}_{\mathrm{er}}$ is calculated with eq 23.

Case 18: The classical approach. Both parameters $h_{w}$ and $k_{\text {efr }}$ are simultaneously estimated, using eq 23 to provide an initial estimate for $k_{\text {etro }}$

Case 2A: The hydrodynamics. The value of $k_{\text {efr }}$ is calculated using eq 23 , and it is used to predict the measured temperature profiles using the boundary conditions described by eq $10 \mathrm{~b}$.

Case $2 B$ : The hydrodynamics. $k_{\text {ert }}$ is the only parameter estimated from measurements of radial and axial ternperature profiles in the nonadiabatic bed, using eq 23 to provide initial estimates, and the boundary conditions described by eq $10 \mathrm{~b}$.

Case 3A: The boundary layer approximation. $k_{\text {err }}$ is calculated with eq 23 , and $h_{w}$ is the only estimated parameter using the boundary conditions described by eq $10 \mathrm{a}$.

Case 3B: The boundary layer approximation. Parameters $h_{w}$ and $k_{\text {eff }}$ are simultaneously estimated, using the boundary conditions described by eq 10a; eq 23 provided initial estimates for $k_{\text {efr. }}$.

In this way, the simulations presented for cases $1 \mathrm{~A}, 2 \mathrm{~A}$, and $3 \mathrm{~A}$ take the value for $k_{\text {off }}$ directly from the adiabatic-wall bed experiments, through eq 23 , whereas in cases $1 \mathrm{~B}, 2 \mathrm{~B}$, and $3 \mathrm{~B}$, this parameter is always estimated. Cases $1 \mathrm{~A}, 1 \mathrm{~B}, 3 \mathrm{~A}$, and $3 \mathrm{~B}$ use $h_{w}$ as a second heat-transfer parmeter, which, in all cases, was estimated. Cases $2 A$ and $2 B$ use the boundary conditions described by eq $10 \mathrm{~b}$, whereas cases $3 \mathrm{~A}$ and $3 \mathrm{~B}$ are boundary layer approximations using the conditions described by eq 10a. Other publications, ${ }^{30,31}$ in which the hydrodynamics is coupled to heat transfer in the packed bed, have used the boundary conditions described by eq 10a; however, it is not clear whether they apply any boundary layer approximation.

The comparison of the numerical simulations for the aforementioned six cases, for low Reynolds numbers $\left(\operatorname{Re}_{\mathrm{p}} \leq 160\right)$, showed that, generally, slightly better predictions of the axial and radial temperature profiles were obtained when $k_{\text {sir }}$ was estimated; however, some of the parameter values obtained were up to $20 \%$ different between cases $A$ and $B$. Figures 7 and 8 show the predicted axial and radial temperature profiles for cases IB, 2B, and 3B.

From the analysis of Figures 7 and 8 ; the following conclusions are pertinent:

(1). The prediction of axial temperature profiles from cases 1B, 2B, and 3B are comparatively similar, and reasonably good.

(2) Predictions of radial temperature profiles at two radial positions are reasonably good; however, those that are closer

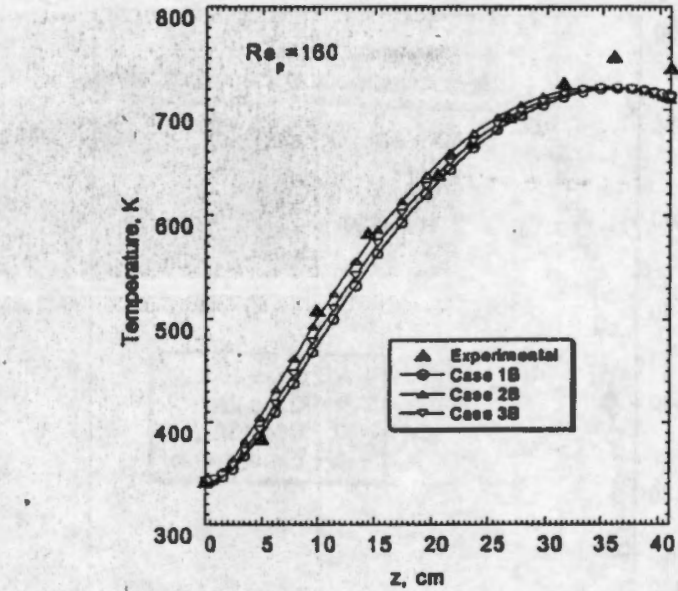

Figure 7. Experimental results compared with predicted axial temperature profiles in the short packed bed.

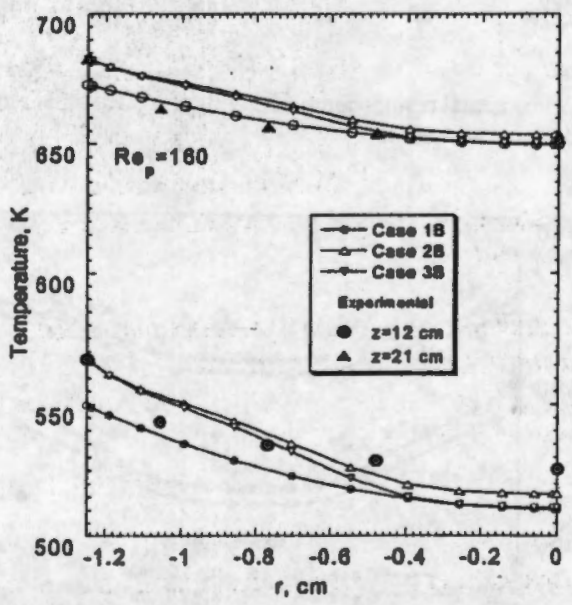

Flgure 8. Experimental results compared with predicted radial temperature profiles in the short packed bed at different axial positions.

to the bed entrance are less satisfactory. On the other hand, the parabolic form of the radial profile is only predicted by the classical approach.

(3) It was determined that predictions at lower Reynolds numbers $\left(R e_{\mathrm{p}}=25\right.$ and 60$)$ were slightly better.

\section{Prediction of Axial and Radial Temperature Profiles in the Nonadiabatic Large-Scale Packed Bed}

The information obtained from the heat-transfer experiments performed in the short adiabatic-wall and nonadiabatic beds can now be transferred to predict the measured radial and axial temperature profiles in the large bed. The objective now is to use this information in the prediction of temperature profiles for the partial oxidation of $o$-xylene with air, performed in the (large-bed) catalytic reactor, ${ }^{22}$ which will be presented in a future communication. As reported previously, the temperature measurements performed in the single-tube industrial-scale catalytic reactor were subjected to distortion, because of the heat losses through the thermowell welded to the reactor tube, through which the thermocouples are inserted. 14,22,71 In the following simulations, no attempt was made to reconstruct the "true" radial temperature profiles from the distorted ones, as in a previous work; ${ }^{14}$ therefore, the predicted symmetrical profiles should be compared to the left portion of the "distorted" radial temperature measurements, which is opposite to the placement of the thermowell, and it is shown at the left side of the figures. 


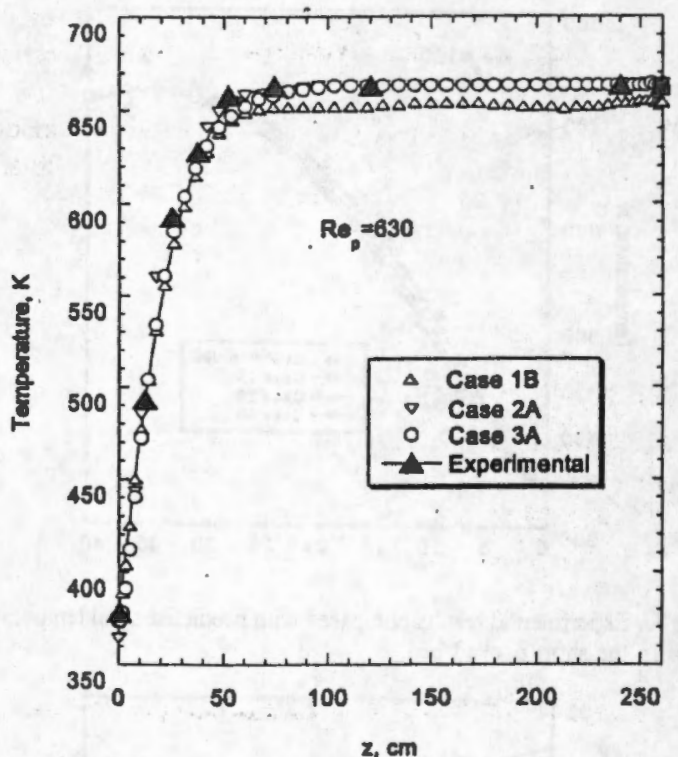

Figure 9. Experimental results compared with predicted axial ternperature profiles in the long packed bod.

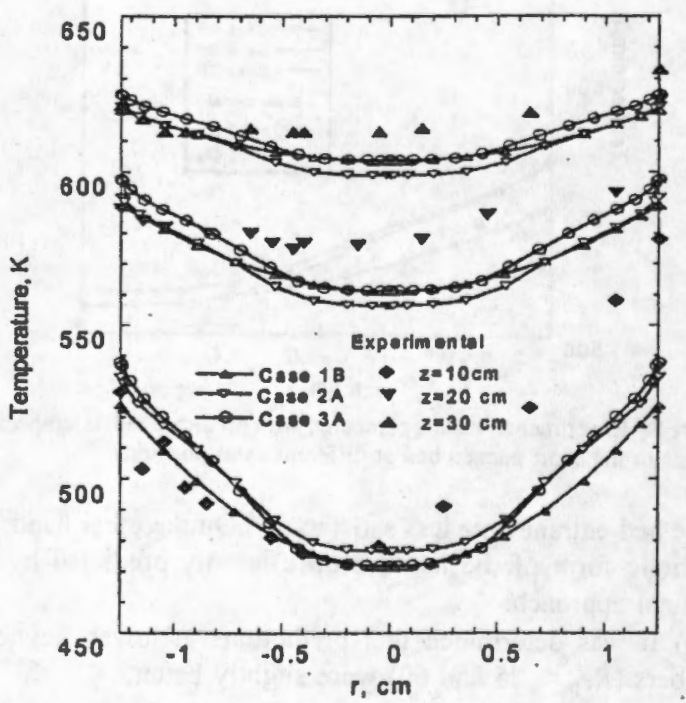

Figure 10. Experimental results compared with predicted radial temperature profiles in the long packed bed at three different axial locations for cases $1 B, 2 A$, and $3 A$.

In this section, the same six cases are compared, but this time, at a higher Reynolds number, $R e_{\mathrm{p}}=630$, which is established by the industrial conditions at which the catalytic reactor normally operates. From the analysis of the simulation results, it was observed this time that better predictions were obtained from cases 1B, 2A, and 3A. Figure 9 compares the experimental data to the predicted axial temperature profiles in the long bed, whereas Figure 10 shows the calculated radial temperature profiles for cases $1 \mathrm{~B}, 2 \mathrm{~A}$, and $3 \mathrm{~A}$. It was determined that case $3 \mathrm{~A}$ was slightly better than case $1 \mathrm{~B}$, and, in this respect, case $2 A$ is free from the uncertainty associated with the use of parameter $h_{w}$, which suggests that the modeling of the heat transport in a packed bed according to case 2 should be preferred.

The comparison of the predictions shown in Figure 10 is not easy, because of the distorted nature of the measurements, as mentioned previously. At this point, one cannot say that including the momentum equations for the axial and radial components of the velocity field in the packed bed model (and a qualitative description of the radial voidage) gives better predictions of the observed axial and radial temperature profiles in a packed bed with low $d / d d_{p}$ ratio at particle Reynolds numbers in the range of $R e_{\mathrm{p}}=160-630$, contrary to the expectations of a recent investigation. ${ }^{29}$

It is observed in Figure 10 that, for the three axial locations shown, the predictions of the radial profiles using the classical approach are as good as those for cases $2 \mathrm{~A}$ and $3 \mathrm{~A}$, which do consider the hydrodynamics. Thus far, the only point in favor of using model 2 is to avoid the uncertainty when parameter $h_{\mathrm{w}}$ is introduced (such as that observed in models of types 1 and 3). One of the limitations of this work in introducing the hydrodynamics in the packed bed is that only two velocity components were considered; moreover, it is expected that, at $R e_{p}=630$, the presence of some turbulence may become important, and this may claim for a turbulence model to describe the packed bed. Finally, the void fraction profiles have been taken only as a function of the tube radius; however, a careful observation of Figure 1 shows that they vary along the three coordinates.

\section{Conclusion}

The estimation of the effective heat-transfer conductivity in packed beds with low tube/particle diameter $\left(d_{V} / d_{p}\right)$ ratio, using experiments in short adiabatic-wall beds, has been successfully used in the prediction of the effective thermal conductivity ( $\left.k_{\text {eft }}\right)$ for short and large nonadiabatic beds of similar dimensions, and it could also be used, with some confidence, in the simulation and design of packed-bed reactors with the same geometrical characteristics. The coupling of hydrodynamics and void fraction profiles to heat transfer in the packed bed makes a good approach to modeling packed-bed catalytic reactors, but it requires measurements at the inner wall temperature; if these are not available, the boundary layer approximation model gives similar predictions. On the other hand, this work has shown that the predictions using the classical approach are as good as those with models that incorporate the hydrodynamics of the packed bed, and no significant improvement in the predictions of the measurements was observed. It has also been shown that the Yagi, Kunii, and Wakao (YKW) correlation, given by eq 23, provides good independent estimates for $k_{\text {itr. }}$ however, parameter $\delta$ in this equation should be estimated from experiments in the packed bed for that particular $d_{1} / d_{p}$ ratio. Although the estimated values of the heat-transfer parameters in the aforementioned six cases are within the expected limits, they have different magnitudes and may produce important differences when used in the simulation and design of a packed-bed catalytic reactor.

\section{Nomenclature}

$B i_{w}=$ wall Biot number; $B i_{w}=R_{T} h_{w} / k_{\text {eff }}$

$C_{\mathrm{pr}}=$ heat capacity of the fluid $(\mathrm{J} /(\mathrm{kg} \mathrm{K}))$

$d_{p}=$ particle diameter $(\mathrm{m})$

$d_{i}=$ tube diameter $(\mathrm{m})$

$g:=$ axial component of gravity $\left(\mathrm{m} / \mathrm{s}^{2}\right)$

$h_{w}=$ wall heat transfer coefficient $\left(\mathrm{W} /\left(\mathrm{m}^{2} \mathrm{~K}\right)\right)$

$K=$ permeability $\left(\mathrm{m}^{2}\right)$

$K_{\xi}=$ pseudo-permeability $\left(\mathrm{m}^{2}\right)$

$k_{\mathrm{eff}}=$ effective thermal conductivity $(\mathrm{W} /(\mathrm{m} \mathrm{K}))$

$k_{\mathrm{e}}^{\circ}=$ static contribution of themal conductivity $(\mathrm{W} /(\mathrm{m} \mathrm{K}))$

$k_{\mathrm{r}}=$ thermal conductivity of the fluid $(\mathrm{W} /(\mathrm{m} \mathrm{K}))$

$L=$ axial length (m) 
$\operatorname{Pr}=$ Prandtl number; $P r=C_{p q} / k_{f}$

$\rho_{\mathrm{r}}=$ radial component of pressure $\left(\mathrm{N} / \mathrm{m}^{2}\right)$

$p_{z}=$ axial component of pressure $\left(\mathrm{N} / \mathrm{m}^{2}\right)$

$P e_{f}=$ fluid Peclet number, $P e_{f}=G C_{p p} R / k_{f}$

$P e=$ Peclet number, $P e=G C_{p p} R_{T} / k_{e f t}$

$P e^{\circ}=$ static contribution of Peclet number, $P e^{\circ}=G C_{p r} R / k_{e}^{\circ}$

$q_{0}=$ heat flux from the infrared lamp $\left(\mathrm{W} / \mathrm{m}^{2}\right)$

$R=$ radial position $(\mathrm{m})$

$R e_{p}=$ particle Reynolds number; $R e_{p}=d_{p} \rho \nu_{0} / \mu$

$R_{\mathrm{t}}=$ tube radius (m)

$T_{\mathrm{w}}=$ wall temperature $(\mathrm{K})$

$T_{0}=$ inlet temperature $(\mathrm{m})$

$T_{\mathrm{b}}=$ salt bath temperature $(\mathrm{K})$

$T=$ temperature $(\mathrm{K})$

$T_{w}=$ steady-state temperature $(\mathrm{K})$

$\nu_{0}=$ volumetric flow rate/cross-sectional area of bed $(\mathrm{m} / \mathrm{s})$

$\nu_{z}=$ axial component of velocity $(\mathrm{m} / \mathrm{s})$

$\nu_{\mathrm{s}}=$ radial component of velocity $(\mathrm{m} / \mathrm{s})$

$z=$ axial position $(m)$

Greek Letters

$\alpha, \beta=$ Ergun parameters

$\delta=$ parameter in the Yagi, Kunii, and Wakao (YKW) correlation 1

$\rho_{\mathrm{f}}=$ density $\left(\mathrm{g} / \mathrm{cm}^{3}\right)$

$\epsilon=$ bed porosity

$\epsilon_{\infty}=$ average bed porosity

$\mu_{\mathrm{f}}=$ dynamic viscosity $\left(\mathrm{g} /\left(\mathrm{cm}^{2} \mathrm{~s}\right)\right)$

\section{Acknowledgment}

This work was supported by El Consejo Nacional de Ciencia y Tecnología de México (under No. SEP-2003-C02-45274-Y).

\section{Literature Cited}

(I) Yagi S.; Kunii D. and Wakao N. Studies on axial effective thermal conductivities in packed beds. AIChE J. 1960, 543 .

(2) Kunii D.; Srnith J. M. Heat transfer characteristics of porous rocks II. AIChE J. 1961, 29.

(3) Vortuba, J.; Hlavacek, V.; Marek, M. Packed bed axial therinal conductivity. Chem. Eng. Sci. 1972, 27, 1845.

(4) Vortmeyer, D.; Adam, W. Steady-state measurements and analytical correlations of axial effective thermal conductivities in packed beds at low gas flow rates. Int. J. Heat Mass Transfer 1984, 27 (9), 1465.

(5) Gunn, D. J.; De Souzan, J. F. C. Heat transfer and axial dispersion in packed beds. Chem. Eng. Sci. 1974, 29 (6), 1363.

(6) Bunnell D. G.; Irvin H. B.; Olson R. W.; Smith J. M. Effective thermal conductivities in gas-solid system. Ind. Eng. Chem. 1949, 4 l (9), 1977.

(7) De Wasch, A. P.; Froment, G. F. Heat transfer in packed beds. Chem. Eng. Sci. 1972, 27, 567.

(8) Snith, J. M. Heal transfer in fixed-bed reactors. Chem. Eng. J. 1973, 5. 109

(9) Lerou. J. J.; Froment, G. F. Velocity, temperature and conversion profiles in fixed bed catalytic reactors. Chem. Eng. Sci. 1977, 32, 853.

(10) Lerou, J. J.; Froment, G. F. Estimation of heat transfer parameters in packed beds from radial temperature profiles. Chem. Eng. J. 1978, 15 (3), 233.

(II) Schlunder. E. U. Transport phenomena in packed bed reactors. In Chemical Reactor Engineering-Houston: The Fifth International Symposium on Chemtcal Reaction Engineering, ACS Symposium Series 65; American Chemical Society (ACS): Washington, DC, 1978; p 110.

(12) Froment, G. F.; Bischoff, K. B. Chemical Reactor Analysis and Design; Wiley: New York, 1979.

(13) Paterson, W. R; Carberry, J. J. Fixed bed catalytic reactor modeling: the heat transfer problem. Chem. Eng. Sci. 1983, 38, 175.

(14) Mongkhonsi T.; López-Isunza H. F.; Kershenbaum L. S. The distortion of measured temperature profiles in fixed-bed reactors. Trans. Inst. Chem. Eng. A 1992, 70, 255.

(15) Dixon, A. G. Heat transfer in fixed beds at very low ( <4) tubeto-particle diameter ratio. Ind. Eng. Chem. Res. 1997, 36, 3053.
(16) Winterberg, M.; Tsotsas, E.; Krischke, A.; Vortmeyer, D. A simple and coherent set of coefficients for modeling of heat and mass transport with and without chemical reaction in tubes filled with spheres. Chem. Eng. Sci. 2000, 55, 967.

(17) Wilhelmus, G. Heat and mass transport in ubular packed bed reactors, Ph. D. Thesis, University of Twente, The Nedherlands, 2002.

(18) Dixon, A. G.; Paterson, W. R.; Cresswell, D. L. Heat transfer in packed beds of low tube/particle diameter ratio. In Chemical Reactor Engineering-Houston: The Fifth Intemational Sympasium on Chemical Reaction Engineering; ACS Symposium Series 65; American Chemical Sociery (ACS): Washington, DC, 1978; p 238.

(19) Dixon, A. G.; Cresswell, D. L. Theoretical prediction of effective heat transfer parameter in packed beds. J. Am. Inst. Chem. Eng. 1979, 25 (4), 663 .

(20) Dixon, A. G.; Cresswell, D. L. Effective heat transfer purameters for transient packed-bed models. J. Am. Inst. Chem. Eng. 1986, 32 (5), 809.

(21) Kershenbaum, L. S.; López-Isunza, F. Dynamic behavior of an industrial scale fixed-bed calalytic reactor. In Chemical Reaction Engineuring Boston; ACS Symposium Series 196; Wei, J., Ed.; American Chemical Society: Washington, DC, 1982.

(22) Lopez-Isunza, F. Steady state and dynamic behavior of an industrial fixed bed catalytic reactor, Ph.D. Thesis, Imperial College, University of London, 1983.

(23) Cresswell, D. L.; Dixon, A. G. Reply to comments by Vortmeyer and Berninger on the paper "Theoretical prediction of effective heat transfer parameters in packed beds (AIChE J., 25, 663, 1979)". AIChE J. 1982, 28 (3), 511.

(24) Tsang, T. H.; Edgar, T. F.; Hougen, J. O. Estimation of heat transfer parameters in a packed bed. Chem. Eng. J. 1976, II, 57.

(25) Michelsen, M. Estimation of heat transfer parameters in packed beds from radial temperauure measurements. Chem. Eng. J. 1979, $18,67$.

(26) Lerou, J. J.; Froment, G. F. Reply to comments by A. G. Dixon and D. L. Cresswell on "Estimation of heal transfer parameters in packed beds from radial temperature profiles". Chem. Eng. J. 1979, 17 (3), 248.

(27) Wakao, N.; Kaguei, S. Heat and Mass Transfer in Packed Bedr; Science Publishers, Ltd.: New York, 1983.

(28) Borman, P. C.; Borkink, J. G. H.; Westerterp, K. R. Heat transport in a wall heated tubular packed bed reactor at elevated pressures and temperatures. Chem. Eng. Commun. 1992, 114, 17.

(29) Wen, D.; Ding, Y. Heat transfer of gas flow through a packed bed. Chem. Eng. Sci. 2006, 61, 3532.

(30) Daszkowski, T.; Eigenberger, G. A re-evaluation of fluid flow, heat trunsfer and chemical reaction in catalyst tilled tubes. Chem. Eng. Sci. 1992. 17, 2245.

(31) Logtenberg. S. A.: Dixon, A. G. Computational fluid dynamics ctudies of the effects of temperature-dependent physical propertion on fixedbed heat transfer. Ind. Eng. Chem. Res. 1998, 37 (3), 739.

(32) Foumeay, E. A.; Ma, J. Non-Darcian non-isothermal compressible flow and heat transfer in cylindrical packed beds. Chem. Eng. Technol. $1994,17,50$.

(33) Guardo, A.; Coussirut, M.; Retasens, F.; Lumrayoz, M. A.; Escaler, $X$. CFD study on particle-to-fluid heat transfer in fixed bed reactors: Convective heat transfer at low and high pressure. Chem. Eng. Sci. 2006, 61,4341 ,

(34) Nijemeisland, M. Verification Studies of Computational Fluid Dynanics in Fixed Bed Heat Transfer, Master Thesis, Faculty of the Worcester Polytechnic Institute (WPI), Worcester, MA, 2000.

(35) Logtenberg, S. A.; Nijemeisland, M.; Dixon, A. G. Computational fluid dynamics simulations of fluid flow and heat transfer at the wallparticle contact points in a fixed-bed reactor. Chem. Eng. Sci. 1999, 54 (13-14), 2433.

(36) Nijemeisland, M.; Dixon, A. G. Comparison of CFD simulations to experiment for convective heat transfer in a gas-solid fixed bed. Chem. Eng. J. 2001, 82. 231

(37) Nijemeisiand, M.; Dixon, A. G.: Stith, E. H. Catalyst design by CFD for heat transfer and reaction in steam reforming. Chem. Eng. Sci. 2004, 59,5185 .

(38) Morales, M.; Spinn, C. W.; Smith, J. M. Velocities and effective thermal conductivities in packed beds. Eng. Processs Dev. 1951, 43 (1), 225.

(39) Cairns, E. J.: Prausnitz, J. M. Velucity profites in packed and fluidized beds. Ind. Eng. Chem. 1959, SI (12), 1441.

(40) Chu, C. F.; $\mathrm{Ng}, \mathrm{K}$. M. Flow in packed bed tubes with small tube to particle dianeter ratio. AIChE J. 1989, 35 (1), 148.

(41) Jasti, J. K.; Fogler, H. S. Determination of flow profiles in porous media using shifts in gamma spectra. AIChE J. 1990, 36 (6), 827.

(42) Bey, O.; Eigenberger, G. Fluid flow and through catalyst tilled tubes. Chem. Eng. Sci. 1996, 52, 1365. 
(43) Giese, M.; Rottshafer, K.; Vortmeyer, D. Measured and modeled superficial tlow profiles in packed beds with liquid flow. A/ChE J. 1998, 44, 484.

(44) Wang, X.; Thauvin, F.; Mohanty, K. K. Non-Darcy flow through anisotropic porous media. Chem. Eng. Sci. 1999, 54, 1859.

(45) Magnico, P. Hydrodynamic and transport properties of packed beds in small tube-to-particle diameter ratio: pore scale simulation using an Eulerian and Lagrangian approach. Chem. Eng. Sei. 2003, 58, 5005.

(46) Freund, H.; Zeiser, T.; Huber, F.; Klemm, E.; Brenner, G.; Durst, F.; Emig, G. Numerical simulations of single phase reacting flows in randomly packed fixed-bed reactors and experimental validation. Chem. Eing. Sci. 2003, 58, 903.

(47) Eisfeld, B.; Schnitzlein, K. A new pseudo-continuous model for the fluid flow in packed beds. Chem. Eng. Sci. 2005, 60, 4105.

(48) Roblee, L. H. S.; Baird, R. M.; Tiemey, J. W. Radial porosity variations in packed beds. $A I C h E, J .1958,4,460$.

(49) Benenati, R. F.; Brosilow, C. B. Void fraction distribution in beds of spheres. AIChE J. 1962, 8 (3), 359.

(50) Winterberg, M.; Tsotsas, E. Impact of tube-to-particle-djameter ratio on pressure drop in packed beds. AIChE J. 2000, 46 (5), 1084.

(51) de Klerk, A. Voidage variation in packed beds at small column to particle diameter ratio. AIChE J. 2003, 49, 2022.

(52) Dixon, A. G. Nijemeisland, M. CFD as a design tool for fixedbed reactors. Ind. Eng. Chem. Res. 2001, 40, 5246.

(53) Calis, H. P. A.; Nijenhuis, J.; Paikert, B. C.; Dautzenberg, F. M.; van den Bleek, C. M. CFD modeling and experimental validation of pressure drop und flow profile in a novel structured casalytic reactor packing. Chem. Eng. Sci. 2001, 56, 1713.

(54) Colburn, A. P. Transfer and pressure drop in empty baffled and packed tubes. Ind. Eng. Chem. 1931, 23 (8), 910.

(55) Kwong, S. S.; Smith, J. M. Radial heat transfer in packed beds. Ind. Eng. Chem. 1953, 49 (5), 894.

(56) Patrick, E. P.; Rinaldo, S. S.; Harriott, P. Heat transfer in packedtube reactors. Ind. Eng. Chem. Res. 1988, 27, 226.

(57) Vortmeyer, D.; Beruinger, R. Comments on the paper, "Theoretical prediction of effective heat transfer parameter in packed beds", by Anthony Dixon and D. L. Cresswell [A/ChE J., 25, 663 (1979)). AIChE J. 1982, 28 (3), 508
(58) Scheidegger A. E. The Physics of Flow through Porous Media; MacMillan: New York, 1957.

(59) Forchheimer, P. Tralado de Hidráulica; Ed. Labor: Barcelona, Spain, 1935.

(60) Brinkman. H. C. A calculation of the viscous force exerted by a flowing fluid on a dense swarm of particles. Appl. Sci. Res. 1947, (AI), 327.

(61) Ergun, S. Fluid flow through packed coiurnns. Chem. Eng. Progress 1952. 48,89 .

(62) Villadsen, J.; Michelsen, M. L. Solution of Differential Equation Models by Poljmomial Appraximation; Prentice Hall: Englewood Cliffs, NJ, 1978.

(63) Finlayson, B. A. Nonlinear Analysis in Chemical Enginetring: MeGraw-Hill: New York, 1980.

(64) Lapidus L.; Seinfeld J. H. Numerical Solution of Ordinary Differential Equations: Academic Press: New York, 1971.

(65) Stewarh W. E.: Caracotsios, M.; Serrensen, J. P. Parameter estimation from multiresponse data. A/ChE J. 1992, 38 (5), 641.

(66) Li, C. H.; Finlayson, B. A. Heat transter in packed beds-a reevaluation. Chem. Eng. Sci. 1977, 32, 1055.

(67) Kulkarni, B. D.; Doraiswamy, L. K. Estimation of effective transport properties in packed bed reactors. Catal. Rev.: Sci. Eng. 1980, 22 (3), 431

(68) Kufner, R.; Hofmann, H. Implementation of radial porosity and velocity distribution in a reactor model for heterogeneous calalytic gas phase reactions. Chem. Eny. Sci. 1990, 8, 2141.

(69) Papageorgiou J. N.; Froment G. F.; Simulation models accounting for radial voidage profiles in tixed-bed reactors. Chem. Eng. Sci. 1995, 50, 3043.

(70) Carberry, 1. J. Private communication, 1980 and 1994.

(71) Sgarlatta Lattmann. Zur Dimensionierung katalytischer festbetreaktoren, Ph.D. Thesis, E.T.H. Zurich, Switzerland, 1969.

Received for review January 16, 2007 Revised manuscript received May 16, 2007 Accepled May 18, 2007 


\title{
INTERNATIONAL JOURNAL OF CHEMICAL REACTOR ENGINEERING

\section{Hydrodynamic Models for Packed Beds with Low Tube-to-Particle Diameter Ratio}

\author{
Carlos O. Castillo-Araiza* Felipe Lopez-Isunza ${ }^{\dagger}$
}

"Universidad Autonoma Metropolitana-Iztapalapa, coca@xanum.uam.mx

tUniversidad Autonoma Metropolitana-Iztapalapa, felipe@xanum.uam.mx ISSN 1542-6580

Copyright (C)2008 The Berkeley Electronic Press. All rights reserved. 


\title{
Hydrodynamic Models for Packed Beds with Low Tube-to-Particle Diameter Ratio
}

\author{
Carlos O. Castillo-Araiza and Felipe Lopez-Isunza
}

\begin{abstract}
In the last decade it has been a special interest to incorporate the hydrodynamics in packed bed reactor models. This seems to be important in the case of highly exothermic partial oxidation reactions normally performed in packed beds with low tube/particle diameter ratio $\left(d_{t} / d_{p}<5\right)$ because of the large void distributions in the radial and axial directions, which have a direct impact on the magnitude of radial, angular and axial profiles of the velocity field, and consequently on both, the temperature and concentration profiles in the catalytic reactor. A successful reactor model needs an adequate hydrodynamic description of the packed bed, and for this reason several models additionally incorporate empirical expressions to describe radial voidage profiles, and use viscous (Darcy) and inertial (Forchheimer) terms to account for gas-solid interactions, via Ergun's pressure drop equation. In several cases an effective viscosity parameter has also been used with the Brinkman's viscous term. The use of these various approaches introduce some uncertainty in the predicted results, as to which extent the use of a particular radial voidage expression, or the use of an effective viscosity parameter, yield reliable predictions of measured velocity profiles.
\end{abstract}

In this work the predictions of radial velocity profiles in a packed bed with low tube to particle diameter ratio from six hydrodynamic models, derived from a general one, are compared. The calculations show that the use of an effective viscosity parameter to predict experimental data can be avoided, if the magnitude of the two parameters in Ergun's equation, related to viscous and inertial energy losses, are re-estimated from velocity measurements, for this particular packed bed. The predictions using both approaches adequately fit the experimental data, although the results are analyzed and discussed.

KEYWORDS: low tube-particle diameter ratio, voidage profiles, hydrodynamic description, effective viscosity, solid-gas interactions 


\section{INTRODUCTION}

The modelling of packed bed reactors for highly exothermic reactions has been performed since mid 1940's (Wilson, 1946; Tasker, 1946); the aim in most cases has been the simulation and design, based on the prediction of the measured concentration and temperature profiles in pilot-scale reactors, assuming that the fluid velocity approaches a uniform radial profile in the packed bed. In the last decade, however, it has been an especial interest to incorporate the hydrodynamics in the packed bed reactor models, which seems to be important for the case of highly exothermic partial oxidation reactions (Lerou and Froment, 1977-1978; Paterson and Carberry, 1983; Lopez-Isunza, 1983; Kufner and Hofinann, 1990; Papageorgiou and Froment, 1995; Anastasov, 2005). Partial oxidation reactions are normally carried out in packed beds with low tube/particle diameter ratio $\left(d / d_{p}<5\right)$, using large catalyst particles $\left(\mathrm{d}_{\mathrm{p}} \sim-8 \mathrm{~mm}\right)$ that create large void distributions in the radial and axial directions (Benenati and Brosilow, 1962; de Klerk 2003). The large variations in voidage in the packed bed have a significant effect on the flow velocity components, which are needed in the prediction of the temperature and concentration profiles for this class of reactors (Lerou and Froment, 1977; Schiunder, 1978; Vortmeyer and Schuster, 1983; Daszkowski and Eigenberger, 1992; Papageorgiou and Froment, 1995; Giese et al., 1998; Winterberg and Tsotsas, 2000).

Several theoretical and experimental studies on velocity profiles within packed beds have been published in the literature (Morales et al., 1951; Lerou and Froment, 1977-78; Foumeny and Ma, 1994; Bey and Eigenberger, 1997; Giese et al., 1998; Wang X. et al., 1999; Magnico, 2003; Freund et. al, 2003; Eisfeld and Schnitzlein, 2005). Large differences in the radial profiles of velocity have been found, because of the uneven distribution of radial voidage; this becomes larger near the reactor wall, particularly in the case of packed beds with low tube/particle diameter ratios. An exact representation of the spatial distribution of the interstitial fluid velocity in packed beds seems to be a very difficult task, because it requires the proper 3D description of the void fraction distribution in the bed at typical values of interest ( $\varepsilon \sim 0.35-0.5)$, as encountered in low tube/particle diameter ratios. For this reason, different empirical equations have been proposed in the literature to describe the observed radial void fraction profiles. On the other hand, the use of Computational Fluid Dynamics codes can define a computational domain that accommodates the geometries of different types of packing into the bed, however the numerical solution of the equations that model the velocity field in the whole packed bed requires large computer resources, and these codes have only been used to solve short packed beds of large voidage, where point contacts among the solid particles seems to be avoided (Logtenberg et al, 1998, 1999; Nijemeisland and Dixon, 2001, 2004; Dixon et al, 2006).

In general, the velocity field is described by a set of modified Navier-Stokes equations that incorporate the fluid-solid interactions with additional coupling terms. The combination of these equations with the pressure drop expression proposed by Ergun (1952), provides these coupling terms, where average quantities replaced the local ones (Chu and Ng, 1989; Foumeny and Ma, 1994; Bey and Eigenberger, 1997; Winterberg and Tsotsas, 2000; Giese et al., 1998). These models also include empirical equations that aim to describe the observed radial voidage, for the calculation of velocity profiles (Bey and Eigenberger, 1997; Giese et al., 1998). Nevertheless, the magnitude of the axial velocity component at the high void fraction near the wall has been generally overestimated (Bey and Eigenberger, 1997; Giese et al., 1998, Eisfeld, 2001; Magnico, 2003). To resolve this problem an "effective viscosity" parameter, following Brinkman (1947), has been introduced in these models to correct for the overestimated magnitude of velocity peaks close to the wall (Bey and Eigenberger, 1997; Winterberg and Tsotsas, 2000; Giese et al., 1998). In this form, the calculations of the observed radial profiles at the exit of the bed, for different types of packing, have been in good agreement with the measurements (Bey and Eigenberger, 1997).

It is known that the magnitude of the Darcy number $(\mathrm{Da})$ gives a good indication of the terms that the momentum equations should include to model the flow in a porous media (JimEnez-Islas et al., 1998). For $\mathrm{Da}<10^{-8}$ the Darcy equation is sufficient to describe the hydrodynamics; for $10^{-8}<\mathrm{Da}<10^{-4}$ the Brinkman term has to be added; for $\mathrm{Da}>10^{-4}$ the inertial terms have to be included. These criteria could be extended to packed-beds with small $d_{1} / d_{p}$, where the bed permeability varies across the tube radius, showing a non-Darcyan behaviour (see Figure 1). In addition to this, this class of packed bed catalytic reactors operate at large particle Reynolds number flows $\left(R_{\text {op }}\right.$ $>600$ ), and because of this an inertial term (Forchheimer, 1935) should also be included in the momentum equations (Foumeny and Ma, 1994).

This study has three aims: the first is to assess the contribution of the viscous and inertial terms that encompass the general momentum equation used in this work; the second is to show that there is no need for 
introducing an extra parameter (the effective viscosity), and at the same time keeping the original parameter values in Ergun's equation (which were obtained for ratios $d_{1} / d_{p} \geq 10$ ), in the calculation of the velocity field in packed beds with ratios $d_{\downarrow} / d_{p} \leq 5$; the third is to show the existing uncertainty in using a particular expression for the voidage profile or the pressure drop correlation, in these predictions. The predictions from the different variants of the general model given here, which have been used previously in the literature, are compared (Chu and $\mathrm{Ng}, 1989$; Bey and Eigenberger, 1997; Foumeny and Ma, 1994; Winterberg and Tsotsas, 2000). In what follows the several simplifications to the general expression of the two-dimension momentum equations of a packed bed are simply called the "hydrodynamic models".

\section{THE HYDRODYNAMIC MODELS}

The velocity measurements used in this study are taken from the work of Bey and Eigenberger (1997), using only the data for the bed packed with spheres at three particle Reynolds number flows; these measurements were taken just below the monolith that supports the packed bed, used to preserve the velocity profile at the outlet of the bed, and the probe is located $5 \mathrm{~mm}$ below the monolith. The position of the probe could be varied in radial an annular directions. These authors also studied the effect of re-packing the bed on the measured velocity profiles. In this study 50 measurements were taken at each location and were averaged; these averaged values are used in our study, for a bed that has $50 \mathrm{~mm}$ internal diameter packed with $9.8 \mathrm{~mm}$ spheres of a non-porous catalyst, with $d_{l} / d_{p} \sim 5$, and average bed porosity of 0.36 , for particle Reynolds numbers: 310,620 , and 930 . This set of data is used here because it is relevant to our current study on comparing different hydrodynamic models for packed bed reactors with low tube to particle diameter ratios.

\subsection{The Void Fraction Profiles}

The porosity profiles in packed beds have been extensively studied, because of its influence on the pressure drop, bed permeability and velocity profiles (Govindarao and Froment, 1986; Govindarao and Ramrao, 1988, 1992; de Klerk, 2003). Several methods have been employed to measure the bed voidage; the most common consists in pouring wax or an epoxy resin into the bed, to maintain the particles in position for the subsequent machining of the radial increments. The void fraction at each section is then calculated from a simple mass balance (Benenati and Brosilow, 1962; Kufner and Hofmann, 1990; Stanek, 1994); other methods have also been developed (Mueller, 1992, Stanek, 1994; de Klerk, 2003). The porosity distribution along the radius in a fixed bed has been normally described by an exponential term for the damping (Vortmeyer and Schuster, 1983) and a cosine term for the oscillations (Kufner and Hoffman, 1990), however Mueller (1992) describes the oscillations with a zero order Bessel function and the damping with an exponential term. Govindarao and Froment (1986) give the voidage variations in terms of the fraction of spheres with centres at cylindrical concentric layers in the bed; however the description becomes less accurate as it moves away from the wall. More recently, a computer model (Nandakumar et al., 1999) has been used to simulate randomly packed beds to investigate voidage variations. However, it has been found that all these models fail to predict the radial oscillation in voidage in beds with low tube to particle diameter ratios.

In this study, the radial void fraction profile in the packed bed is described by the empirical expression given by de Klerk (2003), which considers two regions along the tube radius: one is near the wall, and the other at the core of the bed; these are given by equations (1) and (2). Nevertheless, to show the impact of the void fraction correlation used to predict velocity profiles in a packed bed, the predictions using de Klerk's correlation are compared to that reported by Kufner and Hoffman (1990).

$$
\begin{array}{ll}
\text { For: } & \frac{\left(R_{r}-r\right)}{d_{p}}<0.637 \\
\varepsilon(r)= & 2.14\left(\frac{\left(R_{r}-r\right)}{d_{p}}\right)^{2}-2.53\left(\frac{\left(R_{f}-r\right)}{d_{p}}\right)+1
\end{array}
$$




$$
\begin{aligned}
& \text { For: } \begin{array}{r}
\frac{\left(R_{T}-r\right)}{d_{p}}>0.637 \\
\varepsilon(r)=\varepsilon_{s}+0.29 \exp \left[-0.6 *\left(\frac{\left(R_{T}-r\right)}{d_{p}}\right)\right] *\left(\cos \left(2.3 \pi\left[\frac{\left(R_{T}-r\right)}{d_{p}}-0.16\right]\right)\right) \\
+0.15 \exp \left(-0.9\left[\frac{\left(R_{T}-r\right)}{d_{p}}\right]\right)
\end{array}
\end{aligned}
$$

Figure 1 shows the radial porosity profile calculated with equations (1) and (2) for the data of Bey and Eigenberger (1997); here three zones with large void fraction are observed. The calculated Darcy number along the radial direction is also shown in Figure 1.

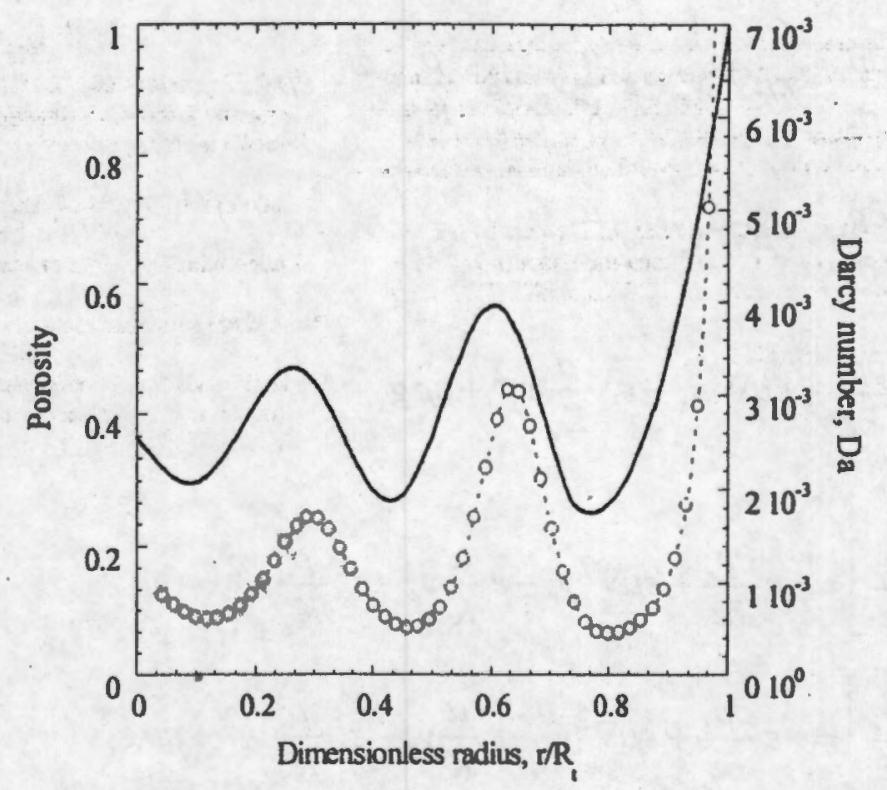

Figure 1. Radial porosity profile $(-)$, and radial Darcy number (0) distribution.

\subsection{The Models}

The models employed in this study have been used before in the literature, and the calculations have shown that there exists a large uncertainty in the prediction of the radial profile of the axial velocity component at the high void fraction regions. As it was mentioned before, to resolve this problem, some researchers have introduced an effective turbulent viscosity parameter ( $\left.\mu_{\text {err }}\right)$ into the Brinkman term, to account for the viscous dissipation effects due to turbulent flow effects at the wall region. This parameter has been estimated for different types of packing, as a function of the tube/particle diameter ratio and the square of the particle Reynolds number (Giese et al., 1998; Bey and Eigenberger, 1997; Winterberg and Tsotsas, 2000). However, $\mu_{\text {off }}$ is normally expressed either in terms of velocity gradients and Prandlt's mixing length (Bird, et, al. 1960), or like those given in an $\varepsilon-k$ turbulence model (Guo et, al. 2003, Dixon et al, 2006). On the other hand, Macdonald et al (1979) have pointed out that Ergun parameters $\alpha$ and $\beta$ (in equations 16 and 17) are not universal constants, and Hicks (1970) claims that these parameters vary with the Reynolds number. For this reason, we believe that it is better to avoid using this extra parameter, and to re-estimate the Ergun constants for each particular bed, from the measured velocity profiles (or pressure drop data), and use only the dynamic viscosity in all the models analysed in this work. Based on this, six different "models" are used to predict the velocity measurements of Bey and Eigenberger (1997); these models are 
derived from model VI given below. Table 1 gives the hydrodynamic models used in this study, assuming that the packed bed behaves as an effective porous medium with constant thermodynamic properties; both viscosity and density correspond to those of a Newtonian fluid, flowing with no slip at the walls.

Table 1. Hydrodynamics models

Model I

$\rho \varepsilon \frac{\partial v_{z}}{\partial t}=-\varepsilon \frac{\partial p_{z}}{\partial z}+\varepsilon \mu \nabla^{2} v_{z}-\varepsilon \frac{\mu}{K} v_{z}+\varepsilon \rho g_{z}$

Model II

$\rho \varepsilon \frac{\partial v_{z}}{\partial t}=-\varepsilon \frac{\partial p_{z}}{\partial z}+\varepsilon \mu \nabla^{2} v_{z}-\varepsilon \frac{\mu}{K} v_{z}-\varepsilon^{2} \frac{\rho}{K_{x}} v_{3}{ }^{2}+\varepsilon \rho g_{z}$

Model III

$\rho \varepsilon\left[\frac{\partial v_{z}}{\partial t}+v_{z} \frac{\partial v_{3}}{\partial z}\right]=-\varepsilon \frac{\partial p_{z}}{\partial z}+\varepsilon \mu \nabla^{2} v_{z}-\varepsilon \frac{\mu}{K} v_{3}-\varepsilon^{2} \frac{\rho}{K_{z}} v_{z}^{2}+\varepsilon \rho g_{3}$

Model IV

$\rho \varepsilon\left[\frac{\partial v_{z}}{\partial t}+v_{z} \frac{\partial v_{z}}{\partial z}\right]=-\varepsilon \frac{\partial p_{z}}{\partial z}+\varepsilon \mu \nabla^{2} v_{z}-\varepsilon \frac{\mu}{K} v_{z}+\varepsilon \rho g_{z}$

Model V

$\rho \varepsilon\left[\frac{\partial v_{z}}{\partial t}+v_{z} \frac{\partial v_{z}}{\partial z}\right]=-\varepsilon \frac{\partial p_{z}}{\partial z}+\varepsilon \mu \nabla^{2} v_{z}-\varepsilon^{2} \frac{\rho}{K_{x}} v_{z}{ }^{2}+\varepsilon \rho g_{z}$

Model VI

Radial velocity:

$\rho \varepsilon\left[\frac{\partial \nu_{r}}{\partial t}+v_{r} \frac{\partial \nu_{r}}{\partial r}+v_{z} \frac{\partial v_{r}}{\partial z}\right]=-\varepsilon \frac{\partial p_{r}}{\partial z}+\varepsilon \mu \nabla^{2} v_{r}-\varepsilon \frac{\mu}{K} v_{r}-\varepsilon^{2} \frac{\rho}{K_{z}} v_{r}^{2}$

Axial velocity:

$\rho \varepsilon\left[\frac{\partial v_{z}}{\partial t}+v_{r} \frac{\partial v_{3}}{\partial r}+v_{z} \frac{\partial v_{z}}{\partial z}\right]=-\varepsilon \frac{\partial p_{z}}{\partial z}+\varepsilon \mu \nabla^{2} v_{z}-\varepsilon \frac{\mu}{K} v_{z}-\varepsilon^{2} \frac{\rho}{K_{z}} v_{z}^{2}+\varepsilon \rho g_{z}$ equations:

Additionally, mass continuity and initial and boundary conditions, given below, form the complete set of

Mass continuity:

$\frac{1}{r} \frac{\partial}{\partial r}\left(r v_{r}\right)+\frac{\partial}{\partial z}\left(v_{z}\right)=0$

The corresponding initial and boundary conditions, depending on the type of model, are:

$$
\begin{array}{ll}
\mathrm{t}=0 & v_{z}(0, r, z)=v_{z s s}(r, z) ; \quad v_{r}(0, r, z)=v_{r s s}(r, z) \\
\mathrm{r}=0 & \frac{\partial v_{z}}{\partial r}=\frac{\partial v_{r}}{\partial r}=0 \\
\mathrm{r}=\mathrm{R}_{1} & v_{z}=v_{r}=0 \\
\mathrm{z}=0 & v_{z}=\left\langle\nu_{z}(r)\right\rangle ; v_{r}=0
\end{array}
$$




$$
\mathrm{Z}=\mathrm{L} \quad \frac{\partial v_{z}}{\partial z}=\frac{\partial v_{r}}{\partial z}=0
$$

Permeability parameters for the packed bed, $K$ and $K_{2}$ are the same as those proposed by Ergun (1952), which contain parameters $\alpha$ and $\beta$ ( $k_{1}$ and $k_{2}$ in Ergun's pressure drop equation), and are given by:

$$
\begin{aligned}
& K=\frac{\varepsilon^{3} d_{p}^{2}}{\alpha(1-\varepsilon)^{2}} \\
& K_{2}=\frac{\varepsilon^{3} d_{p}}{\beta(1-\varepsilon)}
\end{aligned}
$$

The parameter values in Ergun's equation are: $\alpha=150$ and $\beta=1.75$, and several authors (Foumeny and $\mathrm{Ma}$, 1994; Bey and Eigenberger, 1997; Winterberg and Tsotsas 2000) have used these values to predict the velocity profiles in packed beds with smaller $d_{d} / d_{p}$ ratios. Other empirical correlations have also been used, but produce large deviations in the predictions of the pressure drop in beds with low $d_{o} / d_{p}$ at different Reynolds number flows (Eisfeld and Schnitzlein, 2001; Castillo-Araiza et al, 2007). The values of $\alpha$ and $\beta$ change drastically when they are reestimated for low $\mathrm{d}_{\mathrm{w}} / \mathrm{d}_{\mathrm{p}}$ ratios, suggesting there should be larger contributions of the gas-solid interactions, as Hicks (1970) has already noted. Eisfeld and Schnitzlein (2005) have used a modified Ergun's pressure drop correlation to predict the velocity profiles for a bed with $d_{p} / d_{p}=9.3$, however the calculated velocities at the high void fraction regions were also overestimated.

In this work the magnitudes of $\alpha$ and $\beta$ are re-estimated from the velocity profile data from Bey and Eigenberger (1997), and the calculation of velocity profiles are compared to experiments, and to those when $\mu_{\text {eff }}$ is used in the Brinkman term in model III. All models in Table I contain the Brinkman term but using the dynamic viscosity. Models II, III, and VI incorporate the Ergun expression to account for gas-solid interactions in the packed bed. Models I and IV use only the Darcy term; the former ignores the convective term, whereas the latter incorporate this term. Model V accounts for gas-solid interaction using only the Forchheimer term. Model III contains all the terms that appear in models I, II, IV and V. Finally, model VI is the extension of model III to two dimensions, to compute the axial and radial velocity components.

Model III was also used in the calculation of two extra cases. One was to assess the effect of using an effective viscosity in the Brinkman term on the calculated radial velocity profile, together with the original values of the Ergun constants $\left(\alpha\right.$ and $\beta$ ); in this case $\mu_{\text {erf }}$ is the parameter to be estimated from the prediction of the experimental data of Bey and Eigenberger. The second case was to test different equations used in the calculations of the bed radial void fraction and an alternative expression to Ergun's equation. All of these results are compared to those when model III employs the dynamic viscosity in all the viscous terms, and the values of $\alpha$ and $\beta$, from Ergun's equation, were re-estimated to fit the experimental data.

All models were solved by the method of collocation orthogonal using shitted-Legendre polynomials (Villadsen and Michelsen, 1978); the number of interior collocation points was 20 in each coordinate. The resulting set of ordinary differential equations was solved by the Runge Kutta-Fehlberg method (Lapidus and Seinfeld, 1971). The parameters $\alpha, \beta$, and $\mu_{\text {eff }}$ were estimated from the average values of the velocity measurements (Bey and Eigenberger, 1997), using Levenberg-Marquardt method (Stewart et al., 1995).

\section{RESULTS AND DISCUSSION}

The prediction of the evolution of the normalized radial profiles of the axial velocity component during start-up, using models I to VI with Ergun's original parameter values and employing the dynamic viscosity together with de Klerk equation for radial voidage, are shown in Figures 2 and 3; in all cases the steady state was reached in a very short time $(-10 \mathrm{sec})$. Figure 2 shows three axial velocity peaks along the radial axis, just where the larger void 
fractions are located, as shown in Figure 1. It can be observed that these predictions are not good if they are compared to the experimental data. This suggests that the parameter values associated with the viscous and inertial terms describing the interaction among the fluid and solid phases, do not have the correct magnitude. Nevertheless, models II, III and V, as shown in Figures $2 \mathrm{~b}$ and $2 c$, yielded closer values to the experimental data (see Figure 5). Because models I and IV do not contain an inertial (Forchheimer) term, they predict larger velocity peaks than do models II, III and V; the differences among predictions are in the order of $300 \%$. The close similarity in the predictions by models II, III and V suggest that convective and Darcy terms have a negligible contribution in comparison to the rest, and they may be ignored in the hydrodynamic model, however the Forchheimer term seems to be essential to account for the fluid-solid interactions.
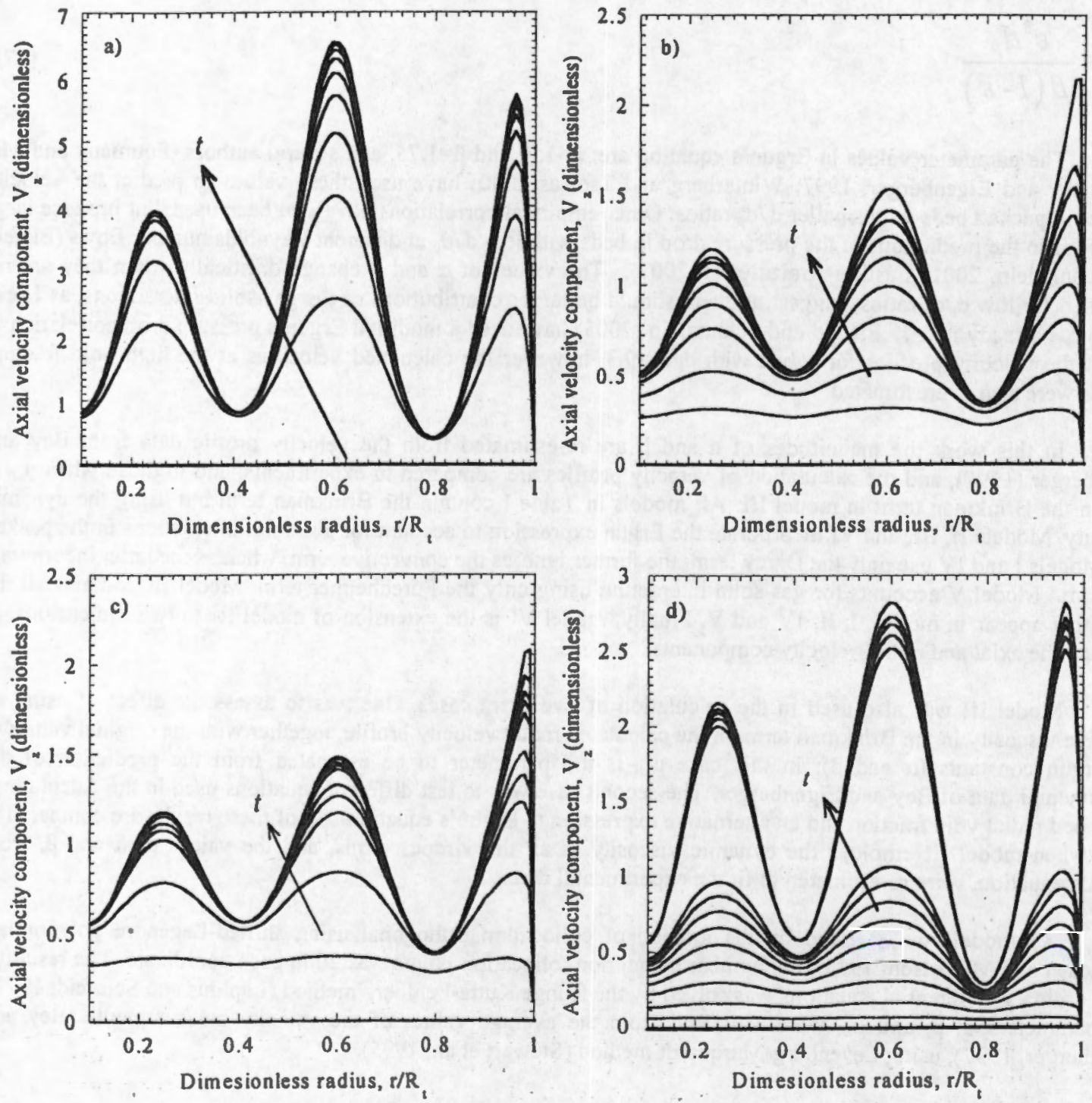

Figure 2. Transients of the dimensionless velocity profiles: a) model I; b) model II; c) models III and V; d) model IV.

Figure 3 shows the axial and radial velocity profiles calculated with model Vl, and it can be seen that the axial velocity component shows the same behaviour as models II, III and V, however the radial velocity component, whose profile is shown only for the first particle layer, becomes zero for the rest of the packed bed length; similar results were found in other works (Daszkowski and Eigenberger, 1992; Papageorgiou and Froment, 1995). These 
results however are the consequence of neglecting in our model the radial pressure gradient, because of the uncertainty in assigning a value to this term.
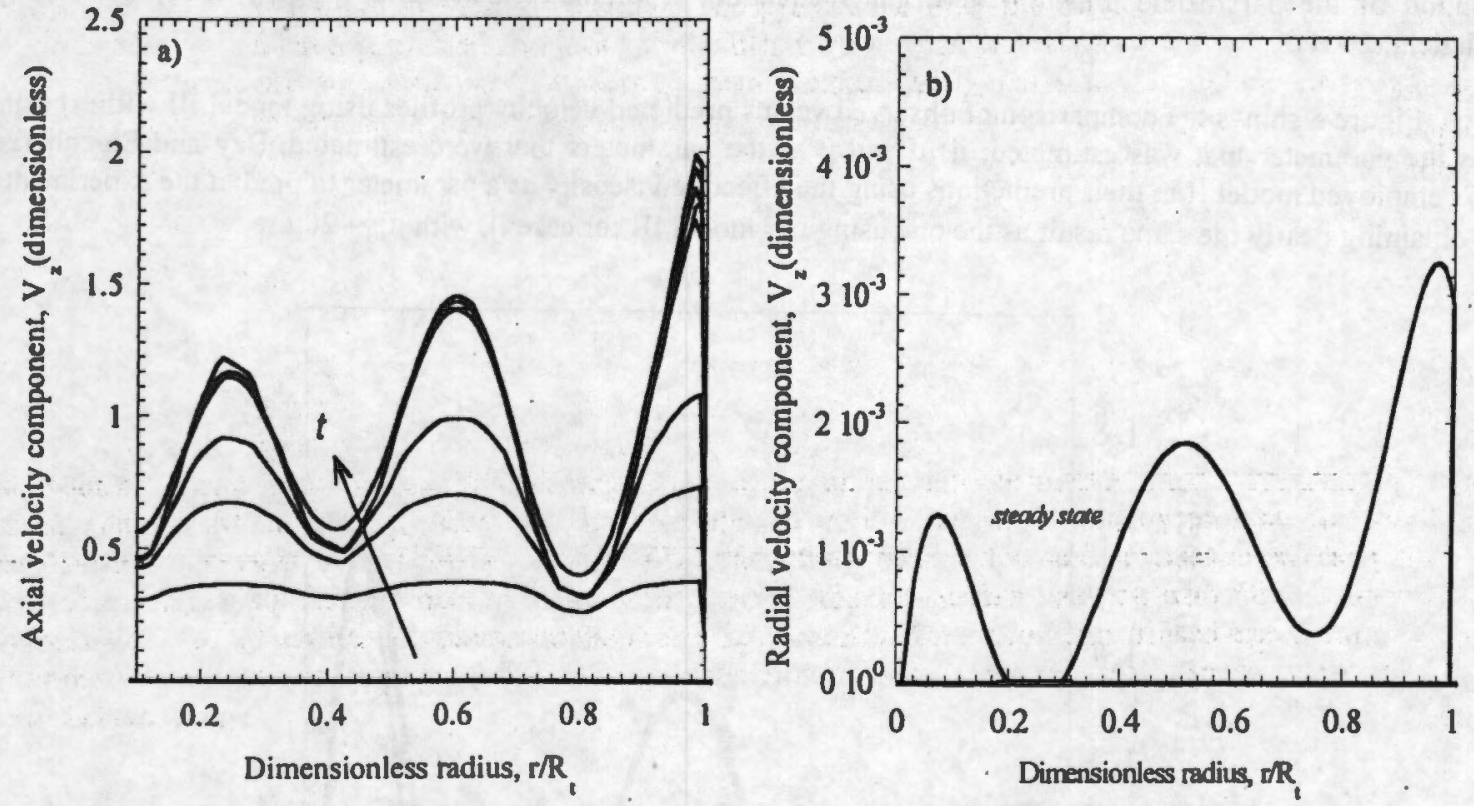

Figure 3. The prediction of the dimensionless velocity profiles: a) axial velocity component; b) radial velocity component

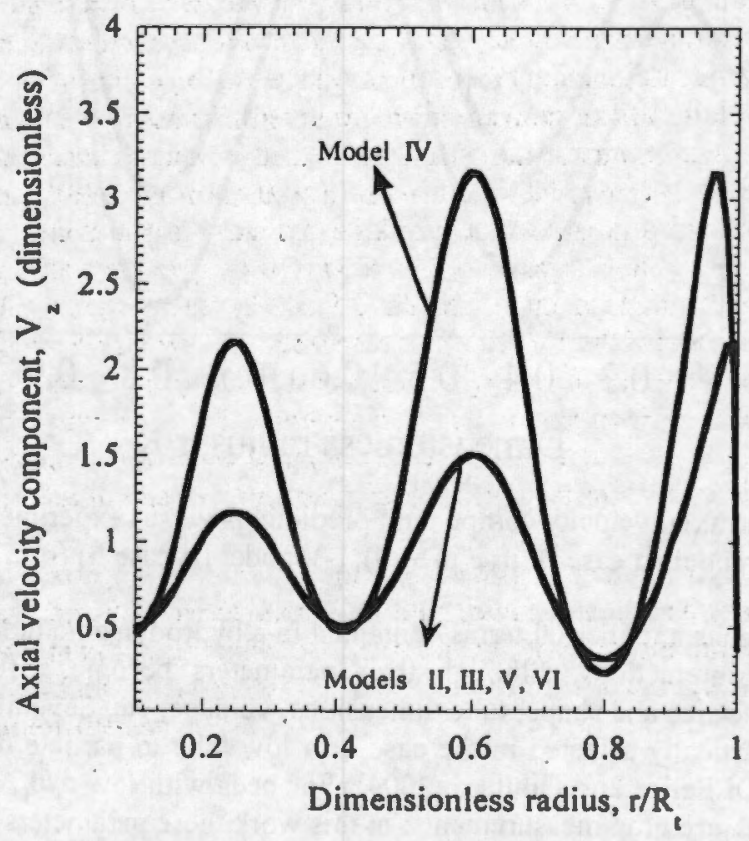

Figure 4. Prediction of the dimensionless axial velocity profiles obtained with models II to VI.

Figure 4 shows the comparison of the steady state velocity profiles calculated with models I to $\mathrm{VI}$; models II, III, V and VI produced practically the same velocity profile, and in all of them the inertial term (Forchheimer) seems to be an essential part in the model, to render closer predictions of the measured velocity profile; models I and IV omit this term, and the calculations with the latter gives, in comparison, rather larger values. However, by 
comparing the predictions from models II, III, V and VI with the measurements, as seen in Figure 5, they still show a poor agreement, overestimating the velocity peaks. This shows the necessity to use, either an empirical parameter like the effective viscosity, or to choose an alternative approach to account for the fluid-solid interactions like the reestimation of the parameters $\alpha$ and $\beta$ in Ergun's equation, from the velocity data (Ergun, 1952; Eisfeld and Schnitzlein, 2001).

Figure $\mathbf{S}$ shows the comparison of observed versus predicted velocity profiles using model III with: i) using $\mu_{\mathrm{eff}}$ as the parameter that was estimated; ii) $\alpha$ and $\beta$ as the parameters that were estimated. Bey and Eigenberger (1997) employed model II in their predictions using the effective viscosity as a parameter to predict the experimental data, obtaining nearly the same result as the one using our model III for case i), with $\mu_{\mathrm{eff}} \sim 20 \mu$.

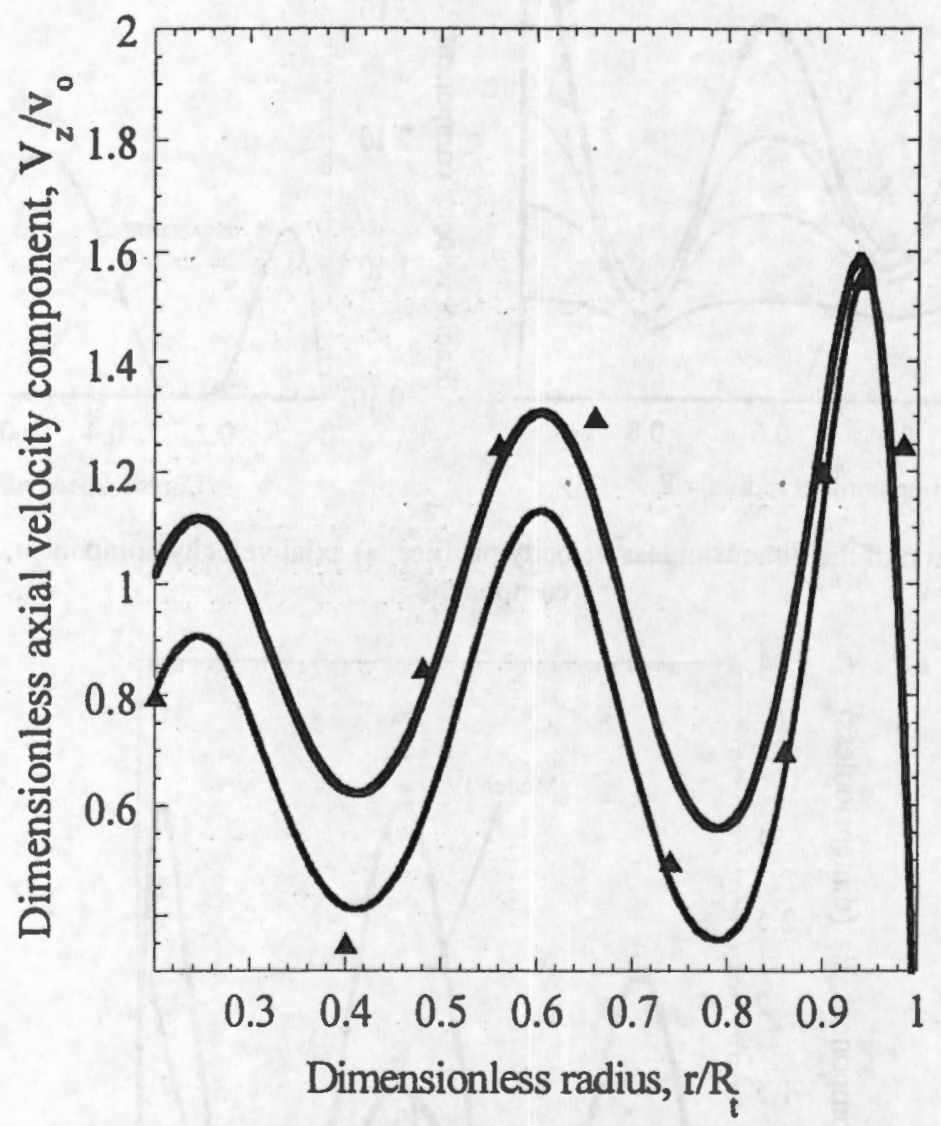

Figure 5. Radial profile of the axial velocity component; predictions versus experimental measurements: (A) Experimental; $(-)$ model III case i) $\left(\mu_{\text {end }} / \mu_{f}=20\right)$; (-) model III case ii) $(\alpha=270$ and $\beta=2.5)$.

It is known that the viscous and inertial terms contained in a hydrodynamic model for packed beds are able to account for the fluid-solid interactions, although their parameters become a function of the geometrical characteristics of the bed: particle size and shape; tube dimensions, voidage, etc. Several authors have found that the magnitude of these terms is drastically affected in the case of a low tube to particle diameter ratio (Ergun, 1952; Eisfeld and Schnitzlein, 2001, Di Felice and Gibilaro, 2004). For beds with low $d_{N} / d_{\rho}$, parameters $\alpha$ and $\beta$ (Ergun) are normally estimated from pressure drop measurements; in this work these parameters were estimated using model III together with the velocity data (Bey and Eigenberger, 1997). The prediction showed in Figure 5 with model III for case ii) yielded estimated values for $\alpha=270$ and $\beta=2.5$.

In this way, the prediction of the measured velocity profiles in packed beds with low tube to particle diameter ratio can be performed, either considering the original parameter values for $\alpha$ and $\beta$ and estimating an effective viscosity term, or via the estimation of $\alpha$ and $\beta$ from velocity measurements. As it is shown in Fig. 5, both 
predictions are very similar and they are very good, although the approach followed in case ii) yields the values for $\alpha$ and $\beta$ that correspond to that particular packed bed, whereas the use of $\mu_{e r}$ only affects the Brinkman term, and therefore it has less information on the fluid-solid interactions that gives the Ergun equation.

To validate our approach using model III, we calculate the normalized axial velocity profiles at three different particle Reynolds numbers, using Bey and Eigenberger (1997) data, together with the re-estimated values of parameters $\alpha$ and $\beta$. Figure 6 compares predictions versus experiments at three Reynolds number flows. In Bey and Eigenberger (1997) paper, the normalized radial velocity profiles, for different flow rates, lie along a single curve (their figures 3 and 8), showing an almost unique profile, when the radial profile of the axial velocity is normalized with respect to the value of the superficial velocity at bed entrance. As it can be observed in Fig. 6, the predictions with model III are also good, validating this approach.

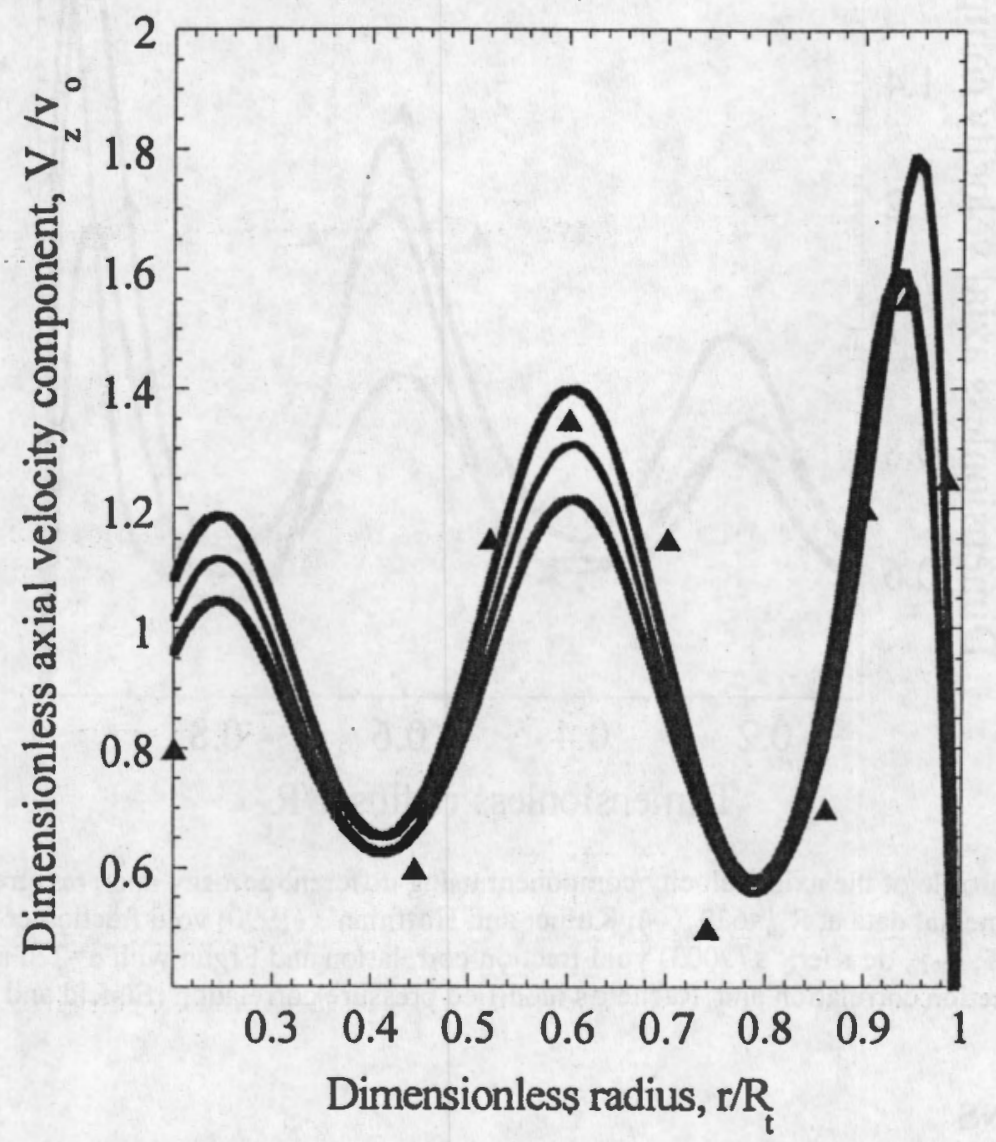

Figure 6. Radial profile of the axial velocity component a three different Reynolds numbers ( $\alpha=270$ and $\beta=2.5$ ). ( $\Delta$ )

$$
\text { Experimental, (-) } R_{e p}=310(-) R_{\text {op }}=620(-) R_{e p}=930
$$

The above results show the same behaviour as those reported by Eisfeld and Schnitzlein (2005), who used the modified Rachelt's correlation (as quoted by Eisfeld and Schnitzlein, 2005) of Ergun's expression, to predict the pressure drop data gathered from the literature. Eisfeld and Schnitzlein showed that this type of correlation could be applied to predict velocity profiles at different particle Reynolds number flows, as well as for different tube/particle diameter ratios. However, as it can be observed in Figure 7, the use of Rachelt's modified correlation, in model III, does not yield good predictions of the velocity profile data for this particular packed bed. Figure 7 also shows the comparison of the predicted radial velocity profiles for the data of Bey and Eigenberger (1997) at $R_{e p}=620$, using different expressions to calculate the radial voidage distribution in the packed bed and Ergun's pressure drop correlation, in model III, for the values of $\alpha$ and $\beta$ used in this work ( $\alpha=270$ and $\beta=2.5$ ). It can be observed that velocity predictions are very sensitive to the use of these expressions in the modelling of the hydrodynamics in a 
packed bed, and that the couple expressions Ergun-de Klerk yielded the best predictions, particularly close to the wall.

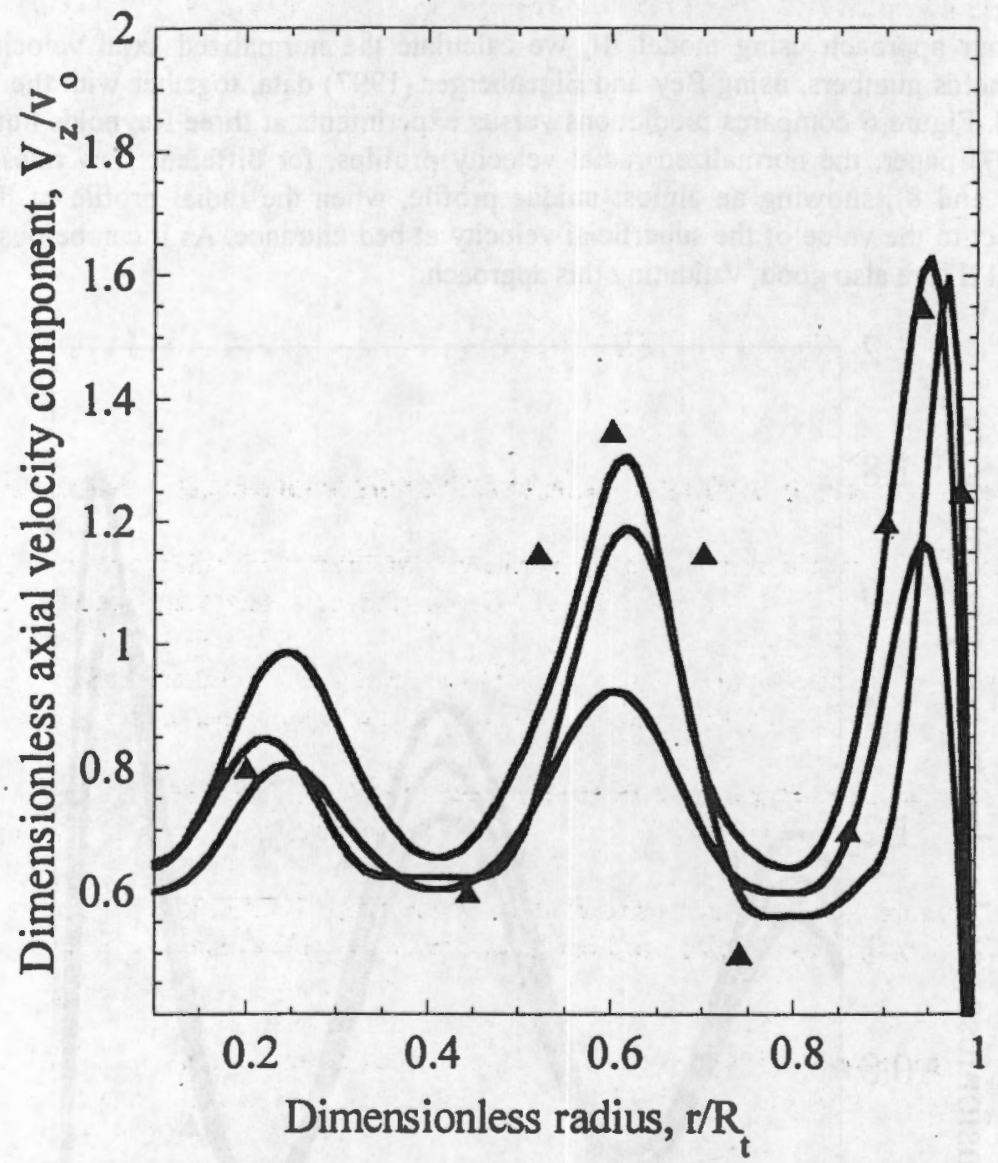

Figure 7. Radial profile of the axial velocity component using different porosity and pressure correlations at $R_{e p}=620$. (4) Experimental data at $R_{e p}=620$ (-); Kufner and Hoffman's (1990) void fraction correlation and Ergun with $\alpha=270$ and $\beta=2.5$; (-) de Klerk's (2003) void fraction correlation and Ergun with $\alpha=270$ and $\beta=2.5$; (-) de Klerk (2003) void fraction correlation and Rachelt's modified pressure correlation (Eisfeld and Schnitzlein (2005))

\section{CONCLUSIONS}

In this work several reduced hydrodynamic models, derived from a general momentum equation, were compared in the prediction of radial velocity profiles in a packed bed with low tube to particle diameter ratio, to assess the contribution of inertial and viscous terms in the general model. Model predictions lead to the following conclusions:

1 Viscous and inertial terms should be included in the hydrodynamic model for the case of packed beds with low tube/particle diameter ratio.

2 The axial viscous dissipation term is important only for the first particles; thereafter the axial velocity becomes constant, in agreement with the experimental measurements obtained by Giese et al. (1998).

3 The radial velocity component has a minor effect after the first particle layer, and it could be ignored, in agreement with other works, unless more information is obtained about the relative magnitude of the radial pressure gradient, with respect to other force terms.

4 For fully developed flow, models II, III, V and VI, for the same set of parameters, predict the same profile. Showing that the inertial term in the Navier-Stokes equation, and the viscous (Darcy) term have little effect in 
the predicted profiles, and the exclusion of the former avoids dealing with excessive computational times.

5 It is expected that good predictions of radial profiles of the axial velocity component could be obtained with models II, III, V and VI, if values of parameters $\alpha$ and $\beta$ are re-estimated from data of velocity or pressure drop measurements for each particular $d / d_{p}$ ratio, this should be preferred than the use of an effective viscosity parameter.

6 The use of correlations given by Ergun (1952) and de Klerk (2003) yielded the best results in the prediction of the velocity profile data used in this work.

\section{ACKNOWLEDGEMENT}

This work was supported by el Consejo Nacional de Ciencia y Tecnología de México (SEP-2003-C02-45274-Y).

\section{NOTATION}

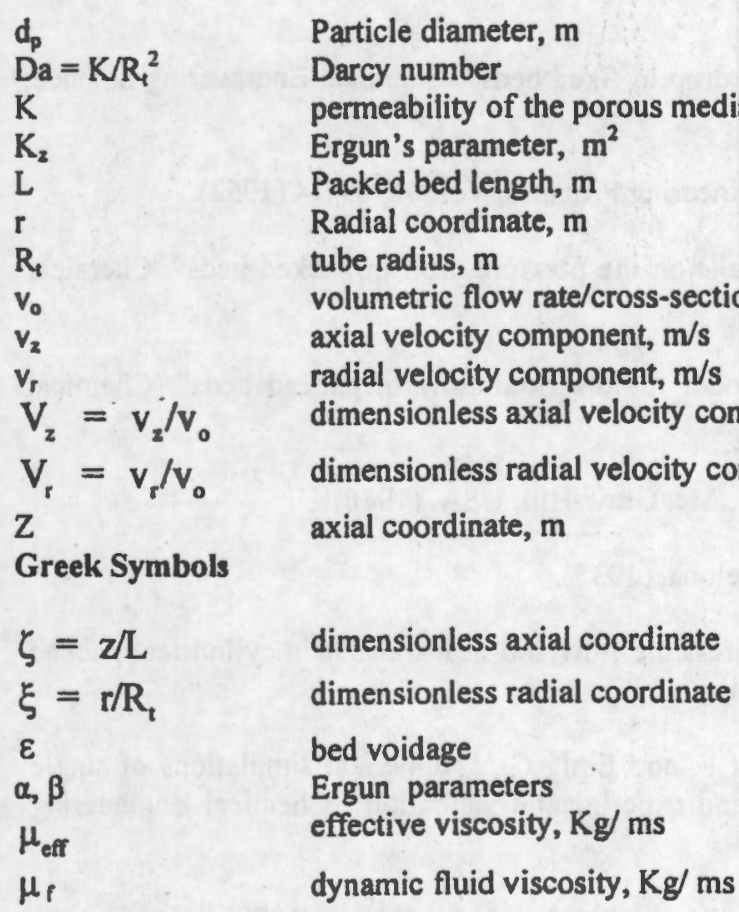

\section{REFERENCES}

Anastasov $\mathrm{A}$., "The behavior of a low-productive non-pretreated $\mathrm{V}_{2} \mathrm{O}_{5}-\mathrm{TiO}_{2}$ (anatase) catalyst for oxidation of oxylene to phthalic anhydride", Chemical Engineering Journal, Vol. 109, Issues 1-3, 57-66 (2005).

Benenati R.F. and Brosilow C.B., "Void fraction distribution in beds of spheres", Journal of American Institute of Chemical Engineers, Vol. 8, No. 3, 359-361 (1962).

Bey O. and Eigenberger G., "Fluid flow through catalyst filled tubes", Chemical Engineering Science, Vol. 52, 1365-1376 (1997).

Bird R. B., Stewart W. E., Lightfoot E. N. "Transport Phenomena", J. Wiley, (1960). 
Brinkman H. C., "A calculation of the viscous force exerted by a flowing fluid on a dense swarm of particles", Applied Scientific Research (A1), 27-34 (1947).

Castillo-Araiza C.; Jimenez-Islas H. and López-Isunza F., "Heat transfer studies in packed bed catalytic reactors of low tube/particle diameter ratio", Industrial and Engineering Chemistry, Vol. 46, 7426-7435 (2007).

Chu C.F. and Ng K.M., "Flow in packed bed tubes with small tube to particle diameter ratio", Journal of American Institute of Chemical Engineers, Vol. 35, No. 1, 148-158 (1989).

Daszkowski T. and Eigenberger G., "A re-evaluation of fluid flow, heat transfer and chemical reaction in catalyst filled tubes", Chemical Engineering Science, Vol. 47, 2245-2250 (1992).

Dixon A. G., Nijmeisland M. and Stitt E. H. "Packed tubular reactor modeling and catalyst design using computational fluid dynamics" Adv. Chem. Eng. Vol. 31, 307- 389 (2006).

de Klerk A., "Voidage variation in packed beds at small column to particle diameter ratios". Journal of American Institute of Chemical Engineers, Vol. 49, 2022-2029 (2003).

Di Felice R. and Gibilaro L.G., "Wall effects for the pressure drop in fixed beds". Chemical Engineering Science, Vol. 59, 3037-3040 (2004).

Ergun S., "Fluid flow through packed columns". Chemical Engineering Progress, Vol. 48, 89-94 (1952).

Eisfeld B. and Schnitzlein K., "The influence of confining walls on the pressure drop in packed beds", Chemical Engineering Science, Vol. 56, 4321-4329 (2001).

Eisfeld B. and Schnitzlein K., "Anew pseudo-continuous model for the fluid flow in packed beds", Chemical Engineering Science, Vol. 60, 4105-4117 (2005).

Finlayson B.A., "Nonlinear Analysis in Chemical Engineering", Mc. Graw-Hill, USA, (1980).

Forchheimer P, "Tratado de Hidráulica", Editorial Labor, Barcelona, (1935).

Foumeny E.A. and Ma J., "Non-Darcian non-isothermal compressible flow and heat transfer in cylindrical packed beds", Chemical Engineering Technology, Vol. 17, 50-60 (1994).

Freund H., Zeiser T., Huber F., Klemm E., Brenner G., Durst F. and Emig G., "Numerical simulations of single phase reacting flows in randomly packed fixed-bed reactors and experimental validation", Chemical Engineering Science, Vol. 58, 903-910 (2003).

Froment G. and Bischoff, K. B. “Chemical Reactor Analysis and Design”, J. Wiley, New York (1979).

Govindrao V.M.H. and Froment G. F., "Voidage profiles in packed beds of spheres", Chemical Engineering Science, Vol. 41, 533-539 (1986).

Govindarao V.M., Ramrao K.V. and Rao A.V., "Structural characteristics of packed bed of low aspect ratio", Chemical Engineering Science, Vol. 47, 2105-2109(1992).

Govindarao V.M. and Ramrao K.V., "Prediction of location of particles in the wall region of a randomly packed bed of spheres", Chemical Engineering Science, Vol. 43, 2544-4545 (1988).

Giese M., Rottschafer K. and Vortmeyer D., "Measured and modelled superficial flow profiles in packed beds with liquid flow", Journal of American Institute of Chemical Engineers, Vol. 44, No.2, 484-490 (1998). 
Guo B., Yu A., Wright B., Zulli P., "Comparison of several turbulence models applied to the simulation of gas flow in a packed bed", Third International Conference on CFD in the Minerals and Process Industries CSIRO, Melbourne, Autralia; pp: 509-514, 10-12 Dec. (2003).

Jiménez-Islas H., "Mathematical Modeling of the momentum, heat and mass transport in porous media", PhD. Thesis (in Spanish) Universidad Autónoma Metropolitana-iztapalapa, México (1998).

Jiménez-Islas H., Ochoa-Tapia J. A. and López-Isunza F. "Natural convection in a cylindrical porous cavity with internal heat source: a numerical study with Brinkman-extended Darcy model", International Journal of Heat and Mass Transfer, Yol. 42, No. 22, 4185-4195 (1999).

Kaviany M., "Principles of Heat Transfer in Porous Media" (2 $2^{\text {nd }}$. Ed) Springer-Verlag, NY (1995).

Kufner R. and Hofmann H., "Implementation of radial porosity and velocity distribution in a reactor model for heterogeneous catalytic gas phase reactions", Chemical Engineering Science, Vol. 8, 2141-2146 (1990).

Lapidus L., Seinfeld J. H., "Numerical Solution of Ordinary Differential Equations", Academic Press, N. Y (1971).

Lerou, J.J. and Froment, G.F., "Velocity, temperature and conversion profiles in fixed bed catalytic reactors", Chemical Engineering Science, Vol. 32, 853-861 (1977).

Lerou, J.J. and Froment, G.F., "Estimation of heat transfer parameters in packed beds from radial temperature profiles", The Chemical Engineering Journal, Vol. 15, No.3, 233-237 (1978).

Lopez-Isunza F., "Steady State and Dynamic Behaviour of an Industrial Fixed Bed Catalytic Reactor", PhD. Thesis, Imperial College, University of London (1983).

Logtenberg S.A. and Dixon A. G., "Computational fluid dynamics studies of the effects of temperature-dependent physical properties on fixed-bed heat transfer", Industrial and Engineering Chemistry Research, Vol. 37, No. 3, 739747 (1998).

Logtenberg S.A., Nijemeisland M. and Dixon A. G., "Computational fluid dynamics simulations of fluid flow and heat transfer at the wall-particle contact points in a fixed-bed reactor", Chemical Engineering Science, Vol. 54 No. $13-14,2433-2439$ (1999).

Macdonald I. F., El-Sayed M. S., Mow K., Dullien A. F. L., "Flow through porous media-the Ergun equation revisited" Ind. Eng. Chem. Fundam., Vol. 18, 199-208 (1979).

Magnico P., "Hydrodynamic and transport properties of packed beds in small tube-to-sphere diameter ratio: pore scale simulation using an Eulerian and a Lagrangian approach", Chemical Engineering Science, Vol. 58, Issue 22, 5005-5024 (2003).

Mueller G.E., "Prediction of radial porosity distribution in randomly packed fixed beds of uniformly sized spheres in cylindrical containers", Chemical Engineering Science, Vol. 46, 706-712 (1991).

Nandakumar K.Y.S and Chuang K.T., "Predicting geometrical properties of random packed bed from computer simulation", Journal of American Institute of Chemical Engineers, Vol. 45, 2286-2292 (1999).

Nijemeisland M. and Dixon A.G., "Comparison of CFD simulations to experiment for convective heat transfer in a gas-solid fixed bed", Chemical Engineering Journal 82, 231-246 (2001).

Nijemeisiand M., Dixon A.G. and Stitt E.H., "Catalyst design by CFD for heat transfer and reaction in steam reforming", Chemical Engineering Science, Vol. 59, 5185-5191 (2004).

Papageorgiou J.N. and Froment G.F., "Simulation models accounting for radial voidage profiles in fixed-bed reactors", Chemical Engineering Science, Vol. 50, 3043-3056 (1995). 
Paterson W.R. and Carberry J.J., "Fixed bed catalytic reactor modeling: the heat transfer problem". Chemical Engineering Science, Vol. 38, 175-180 (1983).

Schlunder E.U., "Transport phenomena in packed bed reactors", Chemical Reactor Engineering- Houston. ACS Symposium Series 65, 110-161 (1978).

Stewart W.E., Caracotsios M. and Sorensen J.P., "Parameter estimation for multiresponse data", GREGPAK (1995).

Stank V., "Fixed Bed Operations", Ellis Horwood, N. Y (1994).

Tasker G. J. H., "The calculation of heat transfer and reaction rate in catalyst beds. Application of theory to phthalic anhydride pilot plant data". Trans. Inst. Chem. Engrs. (London), Vol. 24, 84-89 (1946)

Villadsen J. and Michelsen M. L., "Solution of Differential Equation Models by Polynomial Approximation", Prentice-Hall, Englewood Clifss, N.J (1978).

Vortmeyer D. and Schuster J. "Evaluation of steady flow profiles in rectangular and circular packed beds by a variational method", Chemical Engineering Science, Vol. 38, No. 10, 1691-1699 (1983)

Wang X., Thauvin F. and Mohanty K.K., "Non-Darcy flow through anisotropic porous media". Chemical Engineering Science, Vol. 54, 1859-1869 (1999).

Wilson K. B., "Calculation and analysis of longitudinal temperature gradients in tubular reactors". Trans. Inst Chem. Engrs. (London), Vol. 24, 77-83 (1946)

Winterberg M. and Tsotsas, "Impact of tube-to-particle diameter ratio on pressure drop in packed beds". Journal of American Institute of Chemical Engineers, Vol. 46, No. 5, 1084-1088 (2000).

Winterberg M., Tsotsas E, Krischke A. and Vortmeyer D., "A simple and coherent set of coefficients for modelling of heat and mass transport with and without chemical reaction in tubes filled with spheres", Chemical Engineering Science, Vol. 55, 967-979 (2000). 